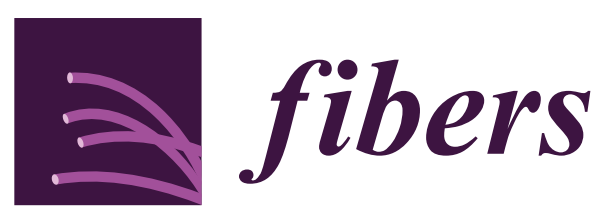

\title{
Nanofibres:
}

\section{Friend or Foe?}


Alke Fink, Barbara Rothen-Rutishauser and

Martin J. D. Clift (Eds.)

\section{Nanofibres: Friend or Foe?}


This book is a reprint of the Special Issue that appeared in the online, open access journal, Fibers (ISSN 2079-6439) from 2014-2016, available at:

http://www.mdpi.com/journal/fibers/special_issues/friend_or_foe

Guest Editors

Alke Fink

(1) BioNanomaterials, Adolphe Merkle Institute, University of Fribourg, Switzerland

(2) Chemistry Department, University of Fribourg, Switzerland

Barbara Rothen-Rutishauser

BioNanomaterials, Adolphe Merkle Institute, University of Fribourg, Switzerland

Martin J. D. Clift

Swansea University Medical School, Singleton Park Campus, Swansea University UK

Editorial Office

MDPI AG

St. Alban-Anlage 66

Basel, Switzerland
Publisher

Shu-Kun Lin
Assistant Editor

Billy Bai

\section{Edition 2016}

MDPI $\bullet$ Basel $\bullet$ Beijing $\bullet$ Wuhan $\bullet$ Barcelona $\bullet$ Belgrade

ISBN 978-3-03842-278-5 (Hbk)

ISBN 978-3-03842-279-2 (electronic)

Articles in this volume are Open Access and distributed under the Creative Commons Attribution license (CC BY), which allows users to download, copy and build upon published articles even for commercial purposes, as long as the author and publisher are properly credited, which ensures maximum dissemination and a wider impact of our publications. The book taken as a whole is (C) 2016 MDPI, Basel, Switzerland, distributed under the terms and conditions of the Creative Commons by Attribution (CC BY-NC-ND) license (http://creativecommons.org/licenses/by-nc-nd/4.0/). 


\section{Table of Contents}

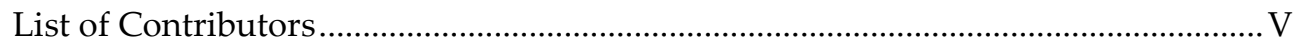

About the Guest Editors............................................................................................. VII

Preface to “Nanofibres: Friend or Foe?" ..................................................................... XI

Alke Petri-Fink, Barbara Rothen-Rutishauser and Martin J. D. Clift

Nanofibers: Friend or Foe?

Reprinted from: Fibers 2016, 4(3), 25

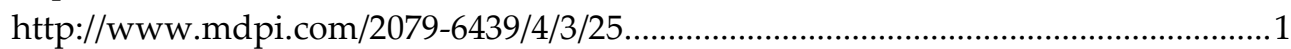

Daisuke Hatanaka, Yasutaka Takemoto, Kazuya Yamamoto and

Jun-ichi Kadokawa

Hierarchically Self-Assembled Nanofiber Films from Amylose-Grafted

Carboxymethyl Cellulose

Reprinted from: Fibers 2014, 2(1), 34-44

http://www.mdpi.com/2079-6439/2/1/34

Matthew S.P. Boyles, Linda C. Stoehr, Paul Schlinkert, Martin Himly and Albert Duschl

The Significance and Insignificance of Carbon Nanotube-Induced Inflammation

Reprinted from: Fibers 2014, 2(1), 45-74

http://www.mdpi.com/2079-6439/2/1/45.

Michel Schaer, Mireille Crittin, Lamia Kasmi, Katarzyna Pierzchala,

Caroline Calderone, Reinaldo G. Digigow, Alke Fink, László Forró and

Andrzej Sienkiewicz

Multi-Functional Magnetic Photoluminescent Photocatalytic Polystyrene-Based

Micro- and Nano-Fibers Obtained by Electrospinning

Reprinted from: Fibers 2014, 2(1), 75-91

http://www.mdpi.com/2079-6439/2/1/75. 
Lukas Schlagenhauf, Frank Nüesch and Jing Wang

Release of Carbon Nanotubes from Polymer Nanocomposites

Reprinted from: Fibers 2014, 2(2), 108-127

http://www.mdpi.com/2079-6439/2/2/108

Jian Yao, Cees W. M. Bastiaansen and Ton Peijs

High Strength and High Modulus Electrospun Nanofibers

Reprinted from: Fibers 2014, 2(2), 158-186

http://www.mdpi.com/2079-6439/2/2/158 .92

Melanie Kucki, Jean-Pierre Kaiser, Martin J. D. Clift,

Barbara Rothen-Rutishauser, Alke Petri-Fink and Peter Wick

The Role of the Protein Corona in Fiber Structure-Activity Relationships

Reprinted from: Fibers 2014, 2(3), 187-210

http://www.mdpi.com/2079-6439/2/3/187

Sandra Camarero-Espinosa, Carola Endes, Silvana Mueller, Alke Petri-Fink, Barbara Rothen-Rutishauser, Christoph Weder, Martin James David Clift and E. Johan Foster

Elucidating the Potential Biological Impact of Cellulose Nanocrystals Reprinted from: Fibers 2016, 4(3), 21

http://www.mdpi.com/2079-6439/4/3/21 154 


\section{List of Contributors}

Cees W. M. Bastiaansen Faculty of Chemistry and Chemical Engineering, Eindhoven University of Technology, P.O. Box 513, 5600 MB Eindhoven, The Netherlands; School of Engineering and Materials Science, and Materials Research Institute, Queen Mary University of London, Mile End Road, London E1 4NS, UK.

Matthew S.P. Boyles Department of Molecular Biology, Paris London-University of Salzburg, Fachbereich Molekulare Biologie, Hellbrunnerstraße 34, Salzburg 5020, Austria.

Caroline Calderone Interdisciplinary Center for Electron Microscopy, School of Basic Sciences, EPFL, Lausanne CH-1015, Switzerland.

Sandra Camarero-Espinosa Australian Institute for Bioengineering and Nanotechnology (AIBN), Cnr College Rd \& Cooper Rd., Building 75, Brisbane, QLD 4072, Australia; Adolphe Merkle Institute, University of Fribourg, Chemin des Verdiers 4, CH-1700 Fribourg, Switzerland.

Martin J. D. Clift Swansea University Medical School, Singleton Park Campus, Swansea, SA2 8PP, Wales, United Kingdom.

Mireille Crittin Institute of Physics of Condensed Matter, School of Basic Sciences, EPFL, Lausanne CH-1015, Switzerland.

Reinaldo G. Digigow Department of Chemistry, and Adolphe Merkle Institute, University of Fribourg, Fribourg CH-1723, Switzerland.

Albert Duschl Department of Molecular Biology, Paris London-University of Salzburg, Fachbereich Molekulare Biologie, Hellbrunnerstraße 34, Salzburg 5020, Austria.

Carola Endes Australian Institute for Bioengineering and Nanotechnology (AIBN), Cnr College Rd \&amp; Cooper Rd., Building 75, Brisbane, QLD 4072, Australia; Adolphe Merkle Institute, University of Fribourg, Chemin des Verdiers 4, CH-1700 Fribourg, Switzerland.

László Forró Institute of Physics of Condensed Matter, School of Basic Sciences, EPFL, Lausanne CH-1015, Switzerland.

E. Johan Foster Department of Materials Science and Engineering, Virginia Tech Center for Sustainable Nanotechnology (VTSuN), Macromolecules Innovation Institute (MII), Virginia Tech, 445 Old Turner Street, 213 Holden Hall, Blacksburg, VA 24061, USA; Adolphe Merkle Institute, University of Fribourg, Chemin des Verdiers 4, CH-1700 Fribourg, Switzerland. 
Daisuke Hatanaka Graduate School of Science and Engineering, Kagoshima University, 1-21-40 Korimoto, Kagoshima 890-0065, Japan.

Martin Himly Department of Molecular Biology, Paris London-University of Salzburg, Fachbereich Molekulare Biologie, Hellbrunnerstraße 34, Salzburg 5020, Austria.

Jun-ichi Kadokawa Research Center for Environmentally Friendly Materials Engineering, Muroran Institute of Technology, 27-1 Mizumoto-cho, Muroran, Hokkaido 050-8585, Japan; Graduate School of Science and Engineering, Kagoshima University, 1-21-40 Korimoto, Kagoshima 890-0065, Japan.

Jean-Pierre Kaiser Empa, Swiss Federal Laboratories for Materials Science and Technology, Laboratory for Materials-Biology Interactions, Lerchenfeldstrasse 5, St. Gallen 9014, Switzerland.

Lamia Kasmi Institute of Physics of Condensed Matter, School of Basic Sciences, EPFL, Lausanne CH-1015, Switzerland.

Melanie Kucki Empa, Swiss Federal Laboratories for Materials Science and Technology, Laboratory for Materials-Biology Interactions, Lerchenfeldstrasse 5, St. Gallen 9014, Switzerland.

Silvana Mueller Adolphe Merkle Institute, University of Fribourg, Chemin des Verdiers 4, CH-1700 Fribourg, Switzerland.

Frank Nüesch Laboratory for Functional Polymers, Swiss Federal Institute for Materials Testing and Research, Dubendorf, Switzerland.

Ton Peijs Nanoforce Technology Ltd., Joseph Priestly Building, and School of Engineering and Materials Science, and Materials Research Institute, Queen Mary University of London, Mile End Road, London E1 4NS, UK.

Alke Petri-Fink Department of Chemistry, University of Fribourg, Switzerland; BioNanomaterials, Adolphe Merkle Institute, University of Fribourg, Chemin des Verdiers 4, CH-1700 Fribourg, Switzerland.

Katarzyna Pierzchala Institute of Physics of Condensed Matter, School of Basic Sciences, EPFL, Lausanne CH-1015, Switzerland.

Barbara Rothen-Rutishauser Adolphe Merkle Institute, University of Fribourg, Chemin des Verdiers 4, CH-1700 Fribourg, Switzerland.

Michel Schaer Institute of Materials, School of Engineering, EPFL, Lausanne CH-1015, Switzerland. 
Lukas Schlagenhauf Institute of Environmental Engineering, ETH Zurich, Zurich, Switzerland; Laboratory for Analytical Chemistry, and Laboratory for Functional Polymers, Swiss Federal Institute for Materials Testing and Research, Dubendorf, Switzerland.

Paul Schlinkert Department of Molecular Biology, Paris London-University of Salzburg, Fachbereich Molekulare Biologie, Hellbrunnerstraße 34, Salzburg 5020, Austria.

Andrzej Sienkiewicz Institute of Physics of Condensed Matter, School of Basic Sciences, EPFL, Lausanne CH-1015, Switzerland.

Linda C. Stoehr GRIMM Aerosol Technik GmbH \& Co. KG, Ainring 83404, Germany.

Yasutaka Takemoto Graduate School of Science and Engineering, Kagoshima University, 1-21-40 Korimoto, Kagoshima 890-0065, Japan.

Jing Wang Institute of Environmental Engineering, ETH Zurich, Zurich, Switzerland; Laboratory for Analytical Chemistry, Swiss Federal Institute for Materials Testing and Research, Dubendorf, Switzerland.

Christoph Weder Adolphe Merkle Institute, University of Fribourg, Chemin des Verdiers 4, CH-1700 Fribourg, Switzerland.

Peter Wick Empa, Swiss Federal Laboratories for Materials Science and Technology, Laboratory for Materials-Biology Interactions, Lerchenfeldstrasse 5, St. Gallen 9014, Switzerland.

Kazuya Yamamoto Graduate School of Science and Engineering, Kagoshima University, 1-21-40 Korimoto, Kagoshima 890-0065, Japan.

Jian Yao School of Engineering and Materials Science, and Materials Research Institute, Queen Mary University of London, Mile End Road, London E1 4NS, UK. 


\section{About the Guest Editors}

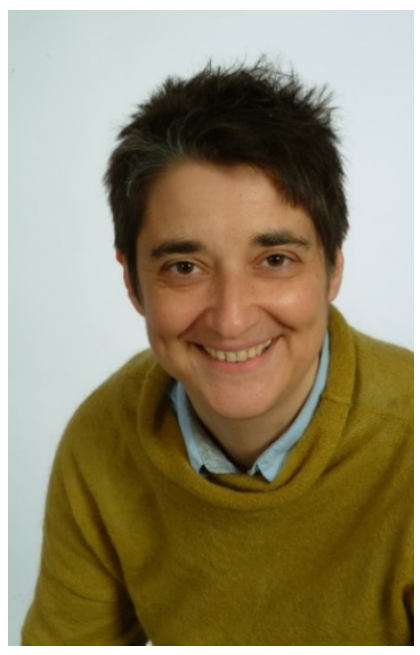

Alke Petri-Fink received her Ph.D. in chemistry from the University of Ulm, Germany in 1999. After a post-doctoral stay at the University of Gainesville, Florida, she joined the Institute of Materials Science at the École Polytechnique Fédérale de Lausanne (EPFL), first as a post-doctoral researcher, then as a senior scientist. She became an Associate Swiss National Science Foundation Professor in the Department of Chemistry at the University of Fribourg in 2009, and Full Professor in 2011 at the Adolphe Merkle Institute, Switzerland. Her research focuses on inorganic nanoparticles, their synthesis, surfaces, and interactions with biological cells. Alke has published more than 140 peer-reviewed papers in addition to several patents, is an invited member of AcademiaNet, a platform for excellent female academics, and serves on several editorial boards.

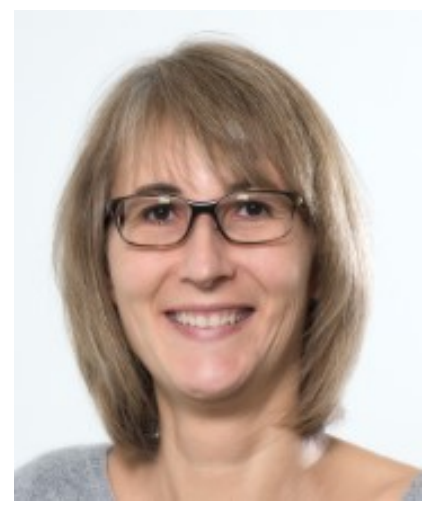

Barbara Rothen-Rutishauser received her Ph.D. in 1996 in cell biology at the Swiss Federal Institute of Technology (ETH) in Zurich. From 1996 to 2000, she held a post-doctoral position in Biopharmacy at the Institute of Pharmaceutical Sciences at the ETH and in 2000, she joined Prof. Peter Gehr's research group at the University of Bern, Switzerland. Barbara Rothen-Rutishauser is an expert in the field of cellnanoparticle interactions in the lung, with a special focus on 3D lung cell models and various microscopy techniques, such as laser scanning and transmission electron microscopy. Since 2011 she is the new chair in BioNanomaterials at the Adolphe Merkle Institute, University of Fribourg, Switzerland, the position is shared equally with Prof. Alke Fink. The research group's activities stretch over many fields from material synthesis and characterization to biological responses and risk assessment. Prof. Rothen-Rutishauser has published more than 190 peer-reviewed papers and is an associate editor of the journal, Particle and Fibre Toxicology. 


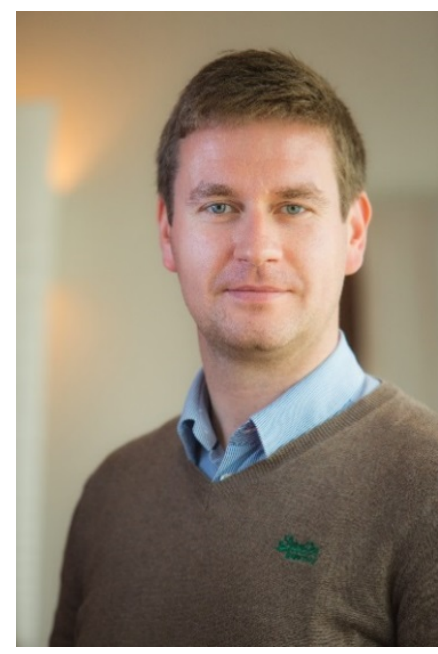

Martin J. D. Clift, a Lecturer at Swansea University Medical School, gained his PhD from Edinburgh Napier University in 2009, followed by 7.5 years' post-doctoral research experience in Switzerland. Using advanced, next-level in vitro systems, Martin's research focuses upon the nanoparticle(mammalian) cell interaction through the use of state-of-the-art microscopy approaches combined with a view towards determining the mechanistic toxicological, immunological and genotoxic effects that nanoparticles, with varying physico-chemcial characteristics, may elicit at the (mammalian) cellular level. Martin is an Associate Editor of the Journal of Nanobiotechnology, and is an editorial board member of Particle and Fibre Toxicology as well as Food and Chemical Toxicology. To date, Martin has authored over 90 publications in the area of in vitro nanotoxicology. Additionally, Martin is a board member of the UK In Vitro Toxicology Society (IVTS), and a member of the UK NC3Rs expert group on nanotoxicology. 



\section{Preface to "Nanofibres: Friend or Foe?"}

Nanofibers, particularly those of a carbonaceous content, have received increased interest in the past two decades due to their outstanding physicochemical characteristics and their possibility to form and contribute towards a plethora of potentially advantageous materials for consumer, industrial and medical applications. Despite this, and together with the numerous research studies and published articles that have sought to investigate these aspects, the potential impact of CNTs is still not understood. Whether or not nanofibers may be able to provide a sophisticated alternative to conventional materials is still debatable, whilst their effects upon both environmental and human health are highly equivocal. How nanofibers are conceived can determine how they may interact with different environments, such as the human body. Understanding each key step of the synthesis and production of nanofibers to their use within potential applications is therefore essential in gaining an insight into how they may be perceived by any biological system and environment. Thus, obtaining such information will enable all scientific communities to begin to realize the potential advantages posed by nanofibers. The aim of this Special Issue therefore, was to provide a collective overview of nanofibers; 'from synthesis to application'. The Issue particularly focuses upon carbon-based nanofibers, but also highlights alternative nanofiber types. Emphasis is given holistically, with articles discussing the production routes of nanofibers, their plight during their life-cycle (origin to applied form and effects over time), as well as how nanofibers could either incite conflict, or provide aid to human and environmental health.

Alke Fink, Barbara Rothen-Rutishauser and Martin J. D. Clift Guest Editors 



\title{
Nanofibers: Friend or Foe?
}

\author{
Alke Petri-Fink, Barbara Rothen-Rutishauser and Martin J. D. Clift
}

Reprinted from Fibers. Cite as: Petri-Fink, A.; Rothen-Rutishauser, B.; Clift, M.J.D. Nanofibers: Friend or Foe? Fibers 2016, 4, 25.

Since the early 1990s nanofibers, particularly those of a carbonaceous content [1] have received heightened interest due to their advantageous physico-chemical characteristics (e.g., high strength, stiffness, semi-conductor, increased thermal conductivity and one of the highest Young's modulus [2]). Such attributes have caused increased debate regarding their potential use as a fundamental component in a wide range of new, advantageous materials for consumer, industrial and medical applications [2]. Yet, concomitantly, due to their dimensions, as well as chemical and elemental structure, concerns as to the human health risk associated with exposure to nanofibers have been vehemently raised [3-5]. Thus, there remains an impending need to undertake research initiatives that focus specifically upon determining the real advantages posed by nanofibers, as well as underpinning their conceivable risk to human health. Both are inextricably linked, and therefore by devising a thorough understanding of the synthesis and production of nanofibers to their potential application and disposal is essential in gaining an insight as to the risk they may pose to human health.

In this Special Issue of Fibers, seven publications (two original articles and four full-length reviews as well as one opinion) are dedicated towards further understanding the nanofibre paradox, notably considering (i) the advantageous structure and mechanical material properties; and (ii) what areas must be considered for future research.

Initially, Yao and colleagues [6], in a paper entitled 'High strength and high modulus electrospun nanofibers', describe, through a detailed review, the ability to create nanoscale continuous fibers via the simple method of electro-spinning. This paper highlights just one of the many possibilities to synthesize nano-sized fibers that elicit high strength and high modulus characteristics, providing essential guidance for future activities in this context. Such future activities are subsequently shown by Schaer et al. [7], who describe the effectiveness of co-encapsulating different forms of nanomaterials (i.e., nanophosphors and superparamagnetic iron oxide nanoparticles) in either polystyrene micro- or nano-fibers using electro-spinning techniques. Through a sophisticated approach, it has been shown that such electro-spun nanomaterials can be used as promising multi-functional magnetic photoluminescent photocatalytic nano-constructs. 
Continuing further, the potential application of nanofibers is then touched upon by Hatanaka et al. [8], who report the ability for cellulose nanofibers, a new and exciting nanofiber type, to form hierarchical self-assembled films. In this original article, which highlights an alternative way of approaching soft nanoscience, it was reported that via an unconventional, bottom-up process, they were able to show that the hierarchically self-assembled nanofibers promoted increased, advantageous level of mechanical properties when under tensile mode.

The context of the Special Issue then changes direction, going from the production and application of nanofibers to the other end of their life-cycle, focusing on the potential release of nanofibers from polymer matrices. In a full-length review, Schlagenhauf and colleagues [9] discuss the ability for carbon nanotubes to be released from polymer nanocomposites under a variety of stress-induced scenarios, including mechanical impact, weathering and fire. This comprehensive article highlights an area of increasing interest within the field of nanotoxicology, especially since the release of nanomaterials in such a scenario would mimic that which humans would be directly exposed to, either accidentally or within an occupational setting.

In context of considering the potential adverse impact of nanofibers upon human health, understanding their physico-chemical characterisation is a must [10]. Recently, in addition to this, the determination as to how nanomaterials interact with their non-cellular, biological environment (i.e., interaction with proteins) has highlighted another avenue of nanomaterial characterisation that will help further deduce their interaction with extra- and intra-cellular entities, such as proteins. Most notably however, understanding how nanomaterials interact with protein complexes has been performed upon spherical-shaped nanomaterials [11], with limited understanding concerning the nanofiber-protein interaction. Therefore, to provide a thorough overview of how proteins interact with fiber-shaped nanomaterials, Kucki et al. [12] highlight recent studies that investigate these complexes and discuss what such interactions may mean towards the hazard potential of nanofibers as well as give indications for future research in this area. Continuing on the theme of the biological impact of nanofibers, Boyles and colleagues [13] discuss the ability for nanofibers to cause inflammation. Focusing upon inhalation exposure, although also touching upon other exposure routes, the effects noted from both in vivo and in vitro research studies following carbon nanotube exposure are discussed. Most notably, this article refers to the potential impact of carbon nanotubes upon the human immune system, and what the consequences of such an interaction might be.

Finally, the Special Issue culminates with an opinion that looks beyond carbon-based nanofibers, specifically nanofibers composed of cellulose. CamareroEspinosa, Endes and Mueller et al. [14] highlight cellulose nanocrystals, a new form of nanofiber receiving increased attention due to their advantageous physical and mechanical characteristics. This opinion-based article is focused towards the essential 
need for attaining knowledge of the biological impact of cellulose nanocrystals, with a special focus upon human health effects. Based upon the view of progressing nanotoxicological assessment of new nanomaterials, the authors providing a strong, yet clear indication as to how future research activities regarding this exciting nanomaterial must be conducted in order to fully comprehend its biological impact (to human health).

In summary, this Special Issue entitled 'Nanofibers: Friend or Foe?' provides significant insight into the nanofiber paradox, with (i) the potential applications posed by nanofibers; and (ii) a discussion of the many issues that remain unresolved in regards to their potential risk towards human health. Discussing major and important components that must be considered within the field, this Special Issue allows for a clear understanding of the problems being encountered combined with a number of definitive solutions as to how to move forward in order to realise the advantages encouraged by these nano-sized materials.

Conflicts of Interest: The authors declare no conflict of interest.

\section{References}

1. Iijima, S. Helical microtubules of graphitic carbon. Nature 1991, 354, 56-58.

2. Robertson, J. Realistic applications of CNTs. Mater. Today 2004, 7, 46-52.

3. Donaldson, K.; Aitken, R.; Tran, L.; Stone, V.; Duffin, R.; Forrest, G.; Alexander, A. Carbon nanotubes: A review of their properties in relation to pulmonary toxicology and workplace safety. Toxicol. Sci. 2006, 92, 5-22.

4. Donaldson, K.; Murphy, F.A.; Duffin, R.; Poland, C.A. Asbestos, carbon nanotubes and the pleural mesothelium: A review of the hypothesis regarding the role of long fibre retention in the parietal pleura, inflammation and mesothelioma. Part. Fibre Toxicol. 2010, 7 .

5. Wick, P.; Clift, M.J.D.; Rosslein, M.; Rothen-Rutishauser, B. A brief summary of carbon nanotubes science and technology: A health and safety perspective. ChemSusChem 2011, 4, 905-911.

6. Yao, J.; Bastiaansen, C.W.M.; Peijs, T. High Strength and High Modulus Electrospun Nanofibers. Fibers 2014, 2, 158-186.

7. Schaer, M.; Crittin, M.; Kasmi, L.; Pierzchala, K.; Caderone, C.; Digigow, R.G.; Fink, A.; Forro, L.; Sienkiewicz, A. Multi-Functional Magnetic Photoluminescent Photocatalytic Polystyrene-Based Micro- and Nano-Fibers Obtained by Electrospinning. Fibers 2014, 2, 75-91.

8. Hatanaka, D.; Takemoto, Y.; Yamamoto, K.; Kadokawa, J.-I. Hierarchically Self-Assembled Nanofiber Films from Amylose-Grafted Carboxymethyl Cellulose. Fibers 2014, 2, 34-44.

9. Schlagenhauf, L.; Nuesch, F.; Wang, J. Release of Carbon Nanotubes from Polymer Nanocomposites. Fibers 2014, 2, 108-127. 
10. Bouwmeester, H.; Lynch, I.; Marvin, H.J.P.; Dawson, K.A.; Berges, M.; Braguer, D.; Byrne, H.J.; Casey, A.; Chambers, G.; Clift, M.J.D.; et al. Minimal analytical characterisation of engineered nanomaterials needed for hazard assessment in biological matrices. Nanotoxicology 2011, 5, 1-11.

11. Cedervall, T.; Lynch, I.; Lindman, S.; Berggard, T.; Thulin, E.; Nilsson, H.; Dawson, K.A.; Linse, S. Understanding the nanoparticle-protein corona using methods to quantify exchange rates and affinities of proteins for nanoparticles. Proc. Natl. Acad. Sci. USA 2007, 104, 2050-2055.

12. Kucki, M.; Kaiser, J.-P.; Clift, M.J.D.; Rothen-Rutishauser, B.; Petri-Fink, A.; Wick, P. The role of the protein corona in fiber structure-activity relationships. Fibers 2014, 2, 187-210.

13. Boyles, M.S.P.; Stoehr, L.C.; Schlinkert, P.; Himly, M.; Duschl, A. The Significance and Insignificance of Carbon Nanotube-Induced Inflammation. Fibers 2014, 2, 45-74.

14. Camarero-Espinosa, S.; Endes, C.; Mueller, S.; Petri-Fink, A.; Rothen-Rutishauser, B.; Weder, C.; Clift, M.J.D.; Foster, E.J. Elucidating the biological impact of nanocellulose. Fibers 2016, 3, 21. 


\title{
Hierarchically Self-Assembled Nanofiber Films from Amylose-Grafted Carboxymethyl Cellulose
}

\author{
Daisuke Hatanaka, Yasutaka Takemoto, Kazuya Yamamoto and \\ Jun-ichi Kadokawa
}

Abstract: In this paper, we report the formation of hierarchically self-assembled nanofiber films from amylose-grafted sodium carboxymethyl celluloses (NaCMCs) that were synthesized by a chemoenzymatic approach. First, maltooligosaccharide primer-grafted $\mathrm{NaCMC}$ were prepared by a chemical reaction using two kinds of NaCMCs with different degrees of polymerization (DPs) from Avicel and cotton sources. Then, phosphorylase-catalyzed enzymatic polymerization of $\alpha$-D-glucose 1-phosphate from the nonreducing ends of the primer chains on the products was conducted to produce the prescribed amylose-grafted NaCMCs. The films were obtained by drying aqueous alkaline solutions of the amylose-grafted NaCMCs. The scanning electron microscopy (SEM) image of the film fabricated from the material with the higher DP from the cotton source showed a clear, self-assembled, highly condensed tangle of nanofibers. The SEM image of the material with the lower DP from the Avicel source, on the other hand, showed an unclear nanofiber morphology. These results indicate that the DPs of the main chains in the materials strongly affected the hierarchically self-assembled nanofiber formation. The SEM images of the films after washing out the alkali, furthermore, showed that the fibers partially merged with each other at the interfacial area owing to the double helix formation between the amylose-grafted chains. The mechanical properties of the films under tensile mode also depended on the self-assembled morphologies of the amylose-grafted NaCMCs from the different sources.

Reprinted from Fibers. Cite as: Hatanaka, D.; Takemoto, Y.; Yamamoto, K.; Kadokawa, J.-I. Hierarchically Self-Assembled Nanofiber Films from AmyloseGrafted Carboxymethyl Cellulose. Fibers 2014, 2, 34-44.

\section{Introduction}

Cellulose is the most abundant biological macromolecule, with a polysaccharide structure consisting of a chain of $\beta$ - $(1 \rightarrow 4)$-linked glucose residues $[1,2]$, and is a very important renewable resource used in furniture, clothing, and medical products. Considerable efforts are also being devoted to developing new material applications of cellulose because of its biodegradable and eco-friendly properties. Self-assembled fibrillar nanostructures from cellulose, so-called nanofibers, are promising 
materials for practical applications in bio-related research fields such as tissue engineering [3-5]. Conventional approaches to the production of cellulose nanofibers are mainly top-down procedures that break down the starting bulk materials from natural cellulose resources [6-8].

In a previous study, we found that the self-assembly of amylose-grafted carboxymethyl cellulose sodium salt (NaCMC) forms nanofiber films upon drying its alkaline aqueous solution [9]. Carboxymethyl cellulose (CMC), an anionic water-soluble polysaccharide, is one of the most widely used cellulose derivatives, and its sodium salt $(\mathrm{NaCMC})$ has a number of $\mathrm{COONa}$ groups that promote water solubility [10]. Our method for the formation of nanofibers from amylose-grafted $\mathrm{NaCMCs}$ is completely different from the aforementioned conventional top-down procedures because our method is a hierarchically self-assembling generative (bottom-up) route, in which fibrillar nanostructures are produced by regeneration from the solutions of the substrates.

Amylose-grafted NaCMC (3) was synthesized by a chemoenzymatic technique according to Scheme 1, which was combined of phosphorylase-catalyzed enzymatic polymerization with chemical reaction [11-19]. Because the enzymatic polymerization of $\alpha$-D-glucose 1-phosphate (G-1-P) is initiated at the nonreducing end of the maltooligosaccharide primer and produces amylose by the following propagation [20-27], the primer was first introduced on the $\mathrm{NaCMC}$ chain by the condensation of an amine-functionalized maltooligosaccharide (1) with carboxylates in NaCMC to give a maltooligosaccharide-grafted NaCMC. Then, the phosphorylase-catalyzed polymerization of G-1-P was conducted using the product to give the prescribed material, 3. The introduction of amylose-graft chains contributed to the construction of a rigid NaCMC main chain, resulting in a nanofiber film upon drying the alkaline solution of the product. Furthermore, the long amylose-graft chains formed double helixes in the intermolecular NaCMC chains by washing out alkali from the film to produce a robust film with the merged nanofiber morphology.

In this paper, we describe the effect of the degree of polymerization (DP) of the $\mathrm{NaCMC}$ main chains on the formation behaviors of hierarchically self-assembled nanofiber films from 3 . For this purpose, the materials were synthesized by the aforementioned chemoenzymatic method using $\mathrm{NaCMC}$ s having similar degrees of carboxymethylation (DC). The $\mathrm{NaCMCs}$ were prepared from two kinds of cellulose with different DPs (microcrystalline cellulose (Avicel No. 2331), DP = ca. 230; cotton, $\mathrm{DP}=c a .2500)[28,29]$. 

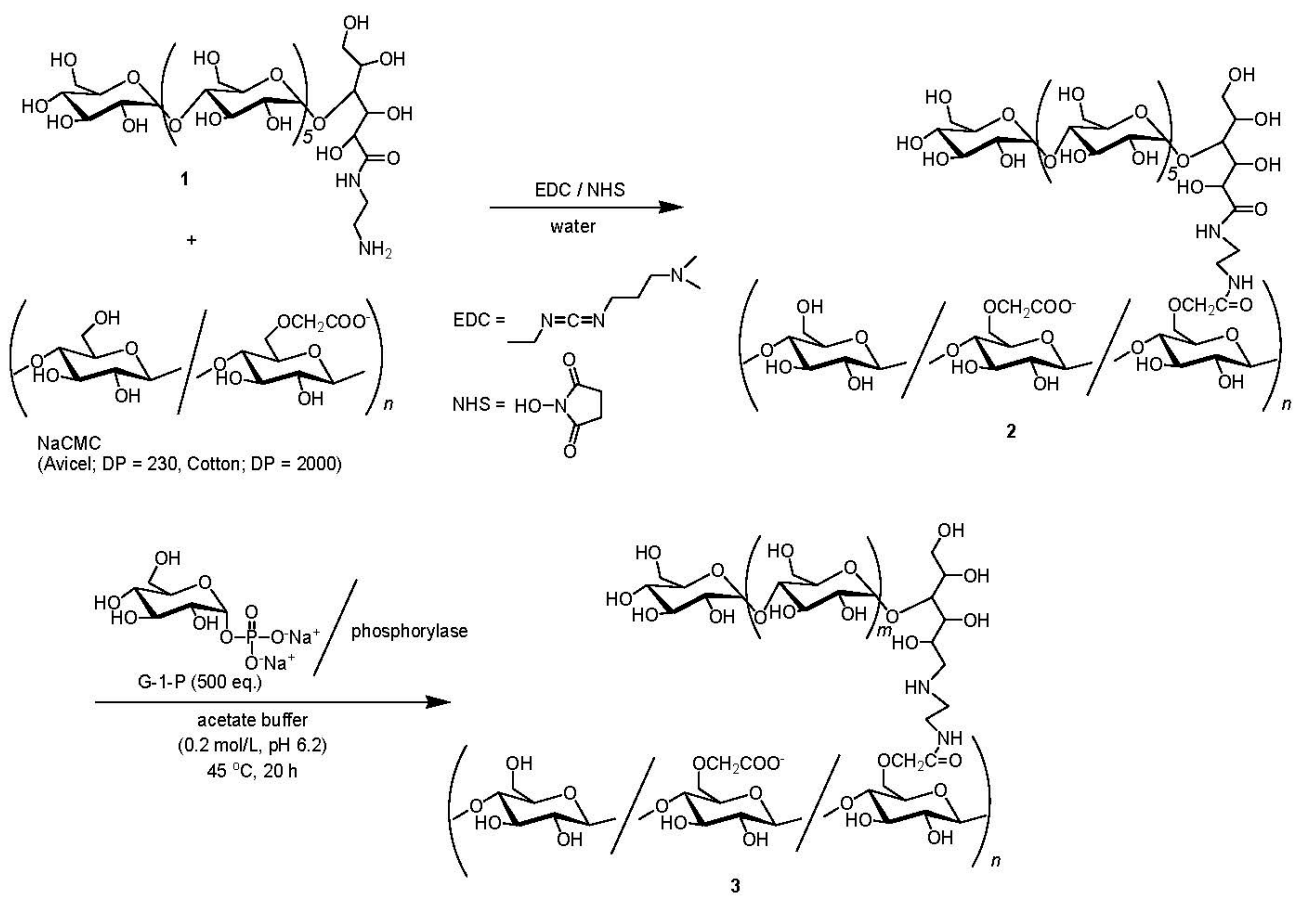

Scheme 1. Chemoenzymatic synthesis of amylose-grafted NaCMC (3).

\section{Experimental Section}

\subsection{Materials}

Microcrystalline cellulose from Merck (Avicel, No. 2331) and absorbent cotton from Kakui Co. Ltd. (Kagoshima, Japan) were used. Carboxymethylation of the cellulose was carried out by the reaction of cellulose with sodium chloroacetate according to the literature procedure [30]. The DC values were estimated by the titration method described in the literature [31]. Thermostable phosphorylase (Aquifex aeolicus VF5) was supplied by Ezaki Glico Co. Ltd., Osaka, Japan [23,32,33]. An amine-functionalized maltooligosaccharide (1) was prepared according to the literature procedure [16]. Other reagents and solvents were used as received.

\subsection{Synthesis of Maltooligosaccharide-Grafted NaCMC (2)}

To a solution of NaCMC (from Avicel, DC $=0.46,0.020 \mathrm{~g}, 0.0101 \mathrm{mmol}$ ) in water $(3.0 \mathrm{~mL})$ was added 1-ethyl-3-(3-dimethylaminopropyl)carbodiimide hydrochloride (EDC) (0.0387 g, $0.202 \mathrm{mmol}$ ) and $\mathrm{N}$-hydroxysuccinimide (NHS) $(0.0232 \mathrm{~g}, 0.202 \mathrm{mmol})$, and the mixture was stirred at room temperature for $1 \mathrm{~h}$. Then, $1(0.245 \mathrm{~g}, 0.202 \mathrm{mmol})$ was added to the solution and the mixture was further 
stirred at room temperature for $24 \mathrm{~h}$. After the reaction solution was dialyzed in a dialysis bag (molecular cut off: 12,000-14,000) against water overnight, the obtained material was purified further by precipitation into methanol $(300 \mathrm{~mL})$. The precipitate was isolated by filtration, washed with dimethyl sulfoxide (DMSO) and methanol, and dried under reduced pressure to give maltooligosaccharide-grafted $\mathrm{NaCMC}$ (2) (0.133 g); ${ }^{1} \mathrm{H} \mathrm{NMR}\left(\mathrm{D}_{2} \mathrm{O}\right) \delta 3.00-4.44$ (sugar protons of $\mathrm{H} 2-\mathrm{H} 6$, $\mathrm{NCH}_{2} \mathrm{CH}_{2} \mathrm{~N}$ ), 4.44-4.68 (H1 of $\mathrm{NaCMC}$ ), 5.17, 5.34 (H1 of maltooligosaccharide). The degree of substitution (DS) for the grafting was determined by the integrated ratio of the $\mathrm{H} 1$ signal of maltooligosaccharide to that of $\mathrm{NaCMC}$ to be 0.074 . Maltooligosaccharide-grafted $\mathrm{NaCMC}$ from cotton was synthesized according to a similar procedure $(\mathrm{DC}=0.43, \mathrm{DS}=0.070)$.

\subsection{Synthesis of Amylose-Grafted NaCMC}

The aforementioned 2 (from Avicel, $0.0080 \mathrm{~g}, 0.0212 \mathrm{mmol}$ ) was dissolved in an aqueous sodium acetate buffer solution $(0.2 \mathrm{~mol} / \mathrm{L}, \mathrm{pH} 6.2,3.0 \mathrm{~mL})$ and G-1-P disodium salt $(0.486 \mathrm{~g}, 1.60 \mathrm{mmol})$ was added to the solution. After the $\mathrm{pH}$ value was adjusted to 6.2 by the addition of $0.2 \mathrm{~mol} / \mathrm{L}$ aqueous acetic acid, thermostable phosphorylase (16 units) was added to this solution, which was then maintained at $45^{\circ} \mathrm{C}$ for $20 \mathrm{~h}$ with stirring. After the resulting gelic mixture was immersed in water $(100 \mathrm{~mL})$ for $3 \mathrm{~h}$, the gel was lyophilized to give amylose-grafted $\mathrm{NaCMC}(3,0.107 \mathrm{~g})$; ${ }^{1} \mathrm{H}$ NMR $\left(1 \mathrm{~mol} / \mathrm{L} \mathrm{NaOD} / \mathrm{D}_{2} \mathrm{O}\right) \delta 3.00-4.44$ (sugar protons of $\mathrm{H} 2-\mathrm{H} 6, \mathrm{NCH}_{2} \mathrm{CH}_{2} \mathrm{~N}$ ), 4.44-4.68 (H1 of NaCMC), 5.13, 5.27 (H1 of amylose). Amylose-grafted NaCMC from cotton was synthesized according to a similar procedure.

\subsection{Formation of Nanofiber Film from 3}

Amylose-grafted NaCMC $3(0.040 \mathrm{~g})$ was first dissolved in a $0.50 \mathrm{~mol} / \mathrm{L} \mathrm{NaOH}$ aqueous solution $(1.5 \mathrm{~mL})$ by stirring the mixture at room temperature. The solution was thinly cast onto a glass plate and dried under ambient conditions to give a film. The resulting film was immersed twice in water $(10 \mathrm{~mL}$ for $10 \mathrm{~min}$ and $5 \mathrm{~mL}$ for $5 \mathrm{~min}$ ) to remove the $\mathrm{NaOH}$ and dried under ambient conditions.

\subsection{Measurements}

${ }^{1} \mathrm{H}$ NMR spectra were recorded on a JEOL ECX-400 spectrometer. Scanning electron microscopy (SEM) images were obtained using an Hitachi SU-70 electron microscope. X-ray diffraction (XRD) measurements were conducted using PANalytical X'Pert Pro MPD with Ni-filtered $C u \mathrm{~K} \alpha$ radiation $(\lambda=0.15418 \mathrm{~nm})$. The stress-strain curves under tensile mode were measured using a tensile tester (Little Senster LSC-1/30, Tokyo Testing Machine). 


\section{Results and Discussion}

\subsection{Chemoenzymatic Synthesis of Amylose-Grafted NaCMC 3}

For the chemoenzymatic synthesis of the amylose-grafted NaCMCs (3), in this study, two kinds of NaCMCs with similar DC values and different DP values were prepared by the carboxymethylation of Avicel and cotton [30]. The NaCMC from Avicel had DC and DP of 0.46 and 230, respectively, and the NaCMC from cotton had DC and DP of 0.43 and 2500, respectively [28,29]. The introduction ofmaltooligosaccharides onto the $\mathrm{NaCMC}$ chains was performed by the condensation of 1 with carboxylates in the NaCMCs using the EDC/NHS condensing agent in water to give 2 (Scheme 1). The ratios of the introduced maltooligosaccharide chains to the repeating units (functionality) in the products from the two kinds of NaCMCs were adjusted to have similar values (DS $=0.074$ from Avicel and DS $=0.070$ from cotton) by appropriate reaction conditions, which were determined by the integrated ratios of the $\mathrm{H} 1$ signal of the maltooligosaccharides to that of the $\mathrm{NaCMCs}$ in the ${ }^{1} \mathrm{H}$ NMR spectra in $\mathrm{D}_{2} \mathrm{O}$.

The amylose-grafted NaCMCs were synthesized by the phosphorylasecatalyzed polymerization of G-1-P from the nonreducing ends of the maltooligosaccharide (primer) graft chains on 2 in the G-1-P/primer feed ratio of 500 (Scheme 1). As the polymerization progressed, the gelation of the reaction mixtures took place. The gelic products were immersed in water and the resulting gels were lyophilized to give 3 . The isolated products were insoluble in water but soluble in aqueous alkaline solution. Thus, the structures of the products were characterized by the ${ }^{1} \mathrm{H}$ NMR spectra measured in $1 \mathrm{~mol} / \mathrm{L} \mathrm{NaOD} / \mathrm{D}_{2} \mathrm{O}$ (Figure 1, from the cotton source), which showed an obvious increase in the integrated ratios of the $\mathrm{H} 1$ signal of amylose to the $\mathrm{H} 1$ signal of $\mathrm{NaCMC}$ as compared with that in the ${ }^{1} \mathrm{H}$ NMR spectra of 2 . The average DPs of the amylose-graft chains in 3 from Avicel and cotton were calculated on the basis of the elemental analysis data, and the functionalities of the maltooligosaccharide chain (the DS values) in 2 were found to be 187 and 218 for Avicel and cotton sources, respectively.

\subsection{Formation and Characterization of Self-Assembled Nanofiber Films from 3}

We previously reported that amylose-grafted NaCMCs (3) with DPs of 140-214 and an amylose-graft chain functionality of 35.4 synthesized from commercially available $\mathrm{NaCMC}(\mathrm{DP}=$ ca. 1200 and $\mathrm{DC}=0.7$ ) formed self-assembled nanofiber films after drying their aqueous alkaline solutions. In the present study, we evaluated the effect of the DPs of the NaCMC main chains in $\mathbf{3}$ on the formation behavior of the self-assembled nanofiber films using two materials synthesized as aforementioned. Solutions of the materials in $0.50 \mathrm{~mol} / \mathrm{L} \mathrm{NaOH}$ aq. were cast onto a glass plate and dried under ambient conditions to give the product films. It was confirmed from the 
SEM image that the film of $\mathbf{3}$ from the cotton source was constructed from nanofibers arranged in a highly condensed tangle (Figure $2 b$ ). On the other hand, such a clear nanofiber morphology was not seen in the SEM image of the film fabricated from the Avicel source, although it showed some broad fibrillar entanglement (Figure 2a). The SEM images of the films after washing out the alkali showed that the fibrils partially merged with each other at interfacial areas with the remaining fibrillar morphologies (Figure 2c,d). The SEM results suggest that the longer NaCMC chain in 3 is favorable for producing the regularly controlled self-assembly needed to construct the clear nanofiber morphology.

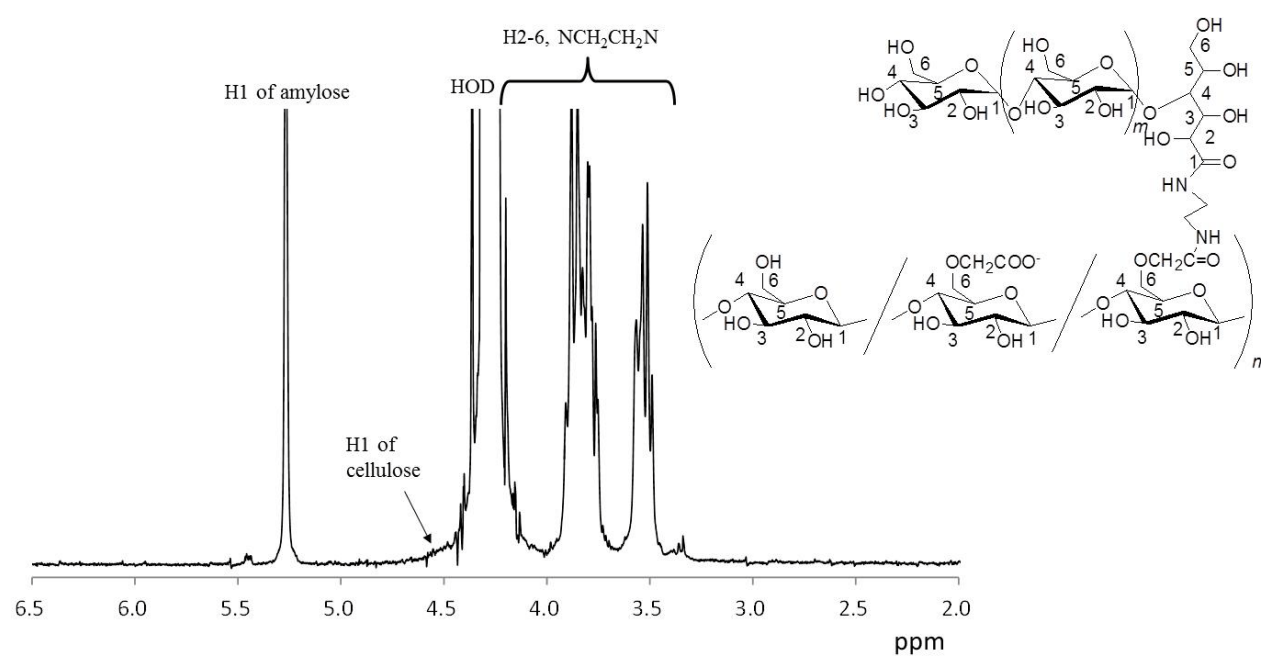

Figure 1. ${ }^{1} \mathrm{H}$ NMR spectrum of amylose-grafted $\mathrm{NaCMC}$ (3) from cotton $\left(\mathrm{NaOD} / \mathrm{D}_{2} \mathrm{O}\right)$.

XRD measurements of the films were conducted to evaluate the hierarchically self-assembled structure of $\mathbf{3}$. The XRD profile of the film of $\mathbf{3}$ from cotton before washing out the alkali slightly show diffraction peaks due to amylose helix at around 17 and $23^{\circ}$ [34] besides $\mathrm{NaOH}$ crystalline peaks (Figure 3a), indicating that the amylose graft chains only partially formed double helix conformation in the film. Because the aqueous alkaline solution is a good solvent for amylose, the formation of an amylose double helix is mostly prevented during the drying process of the solution. After washing out the alkali from the film, the XRD profile exhibited diffraction peaks obviously due to the amylose helix (Figure 3b,c), indicated with shadows), suggesting the progress of double helix formation during the washing process. 

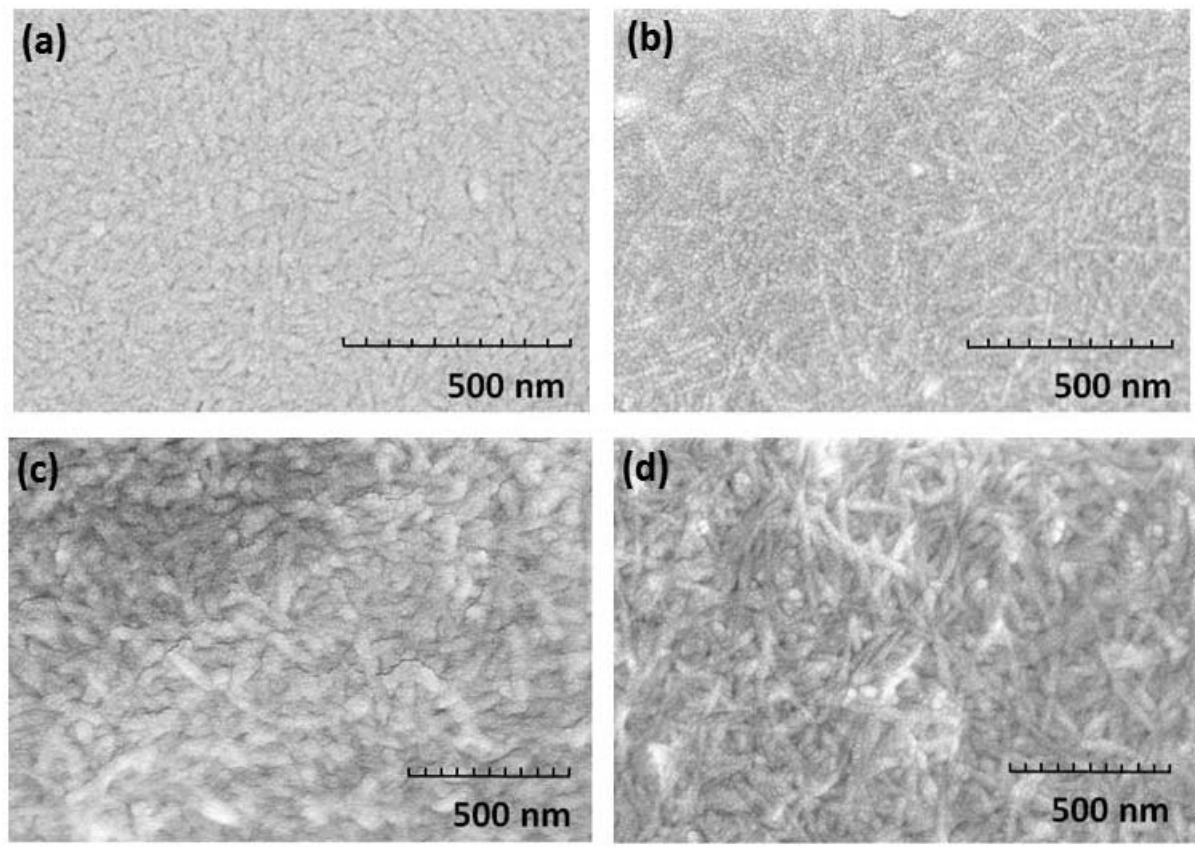

Figure 2. SEM images of films prepared from alkaline solutions of $\mathbf{3}$ from Avicel and cotton $((\mathbf{a}, \mathbf{b})$, respectively) and respective films after washing out alkali $((\mathbf{c}, \mathbf{d})$, respectively).

On the basis of the above results, the self-assembling process of $\mathbf{3}$ is proposed to lead to the formation of the nanofiber film (Figure 4). As already reported in our previous paper [9], the introduction of saccharide-graft chains on NaCMC prevented the construction of a random-coil conformation, resulting in the rigid nature of the $\mathrm{NaCMC}$ chain. While drying the aqueous alkaline solution of 3, some of these rigid materials regularly assembled to induce nanofibrillation with the slight double helix formation from amylose graft chains on NaCMCs, but they did not construct large aggregates because the double helix formation of the most of the amylose chains was prevented due to the alkaline conditions. By washing out alkali from the film, the double helix was able to form on the nanofibers, leading to merging on the surface of the fibers. The average DP value of the NaCMC main chain of 3 from the cotton source (ca. 2500) was much larger than that of the amylose-graft chain (218), resulting in nanofibrillation with a high aspect ratio. On the other hand, because the two DP values of the main and graft chains in $\mathbf{3}$ from the Avicel source were comparable (230 and 187), the self-assembled nanofibers were not clearly formed. 


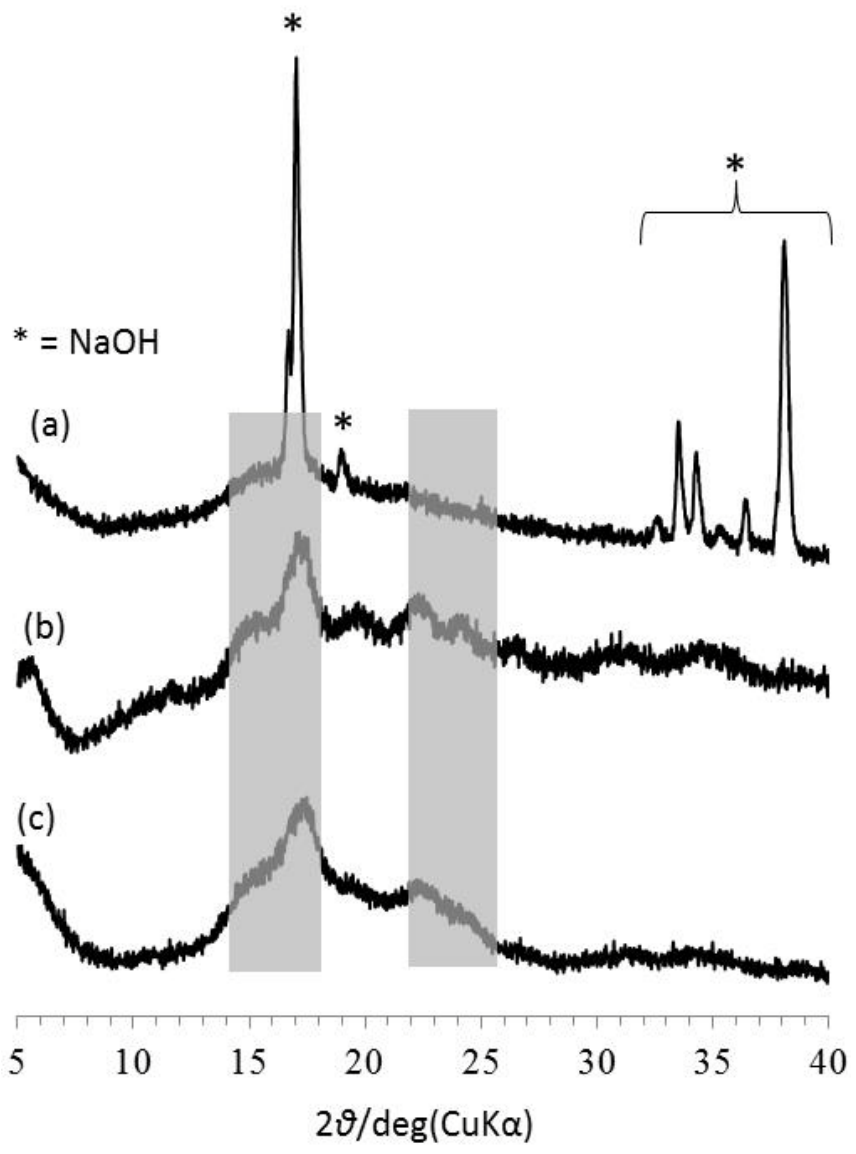

Figure 3. XRD profiles of films of $\mathbf{3}$ from cotton (a) before and (b) after washing out the alkali and (c) amylose.

Finally, the mechanical properties of the films of 3 from the Avicel and cotton sources after washing out alkali were evaluated by tensile testing (Figure 5). The stress-strain curve of the film from the cotton source showed larger fracture stress and strain values than the film from the Avicel source. This result indicates that the DP of the main chain in $\mathbf{3}$ strongly affects the mechanical properties of the present nanofiber film. 


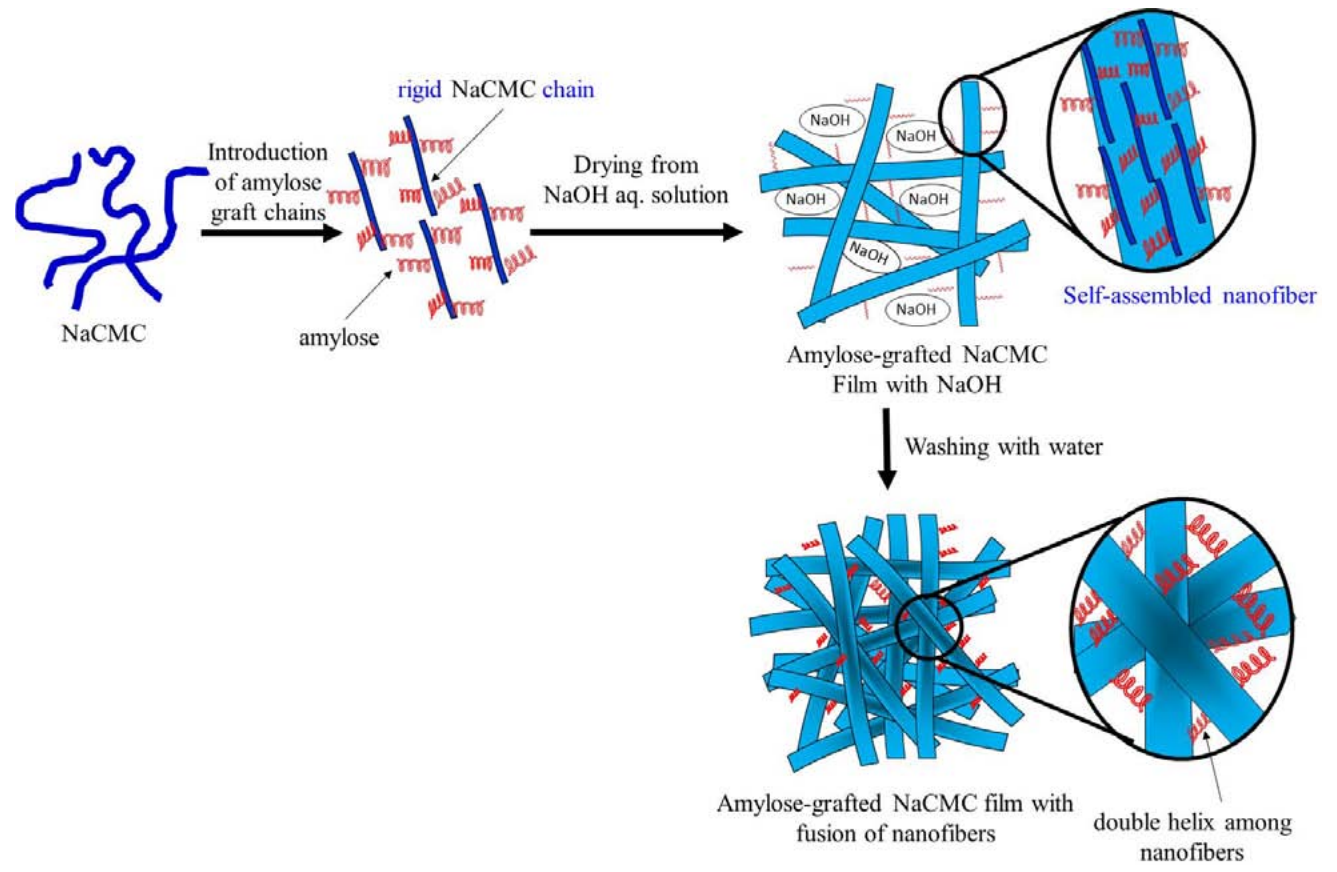

Figure 4. Proposed self-assembling process of 3 under alkaline conditions leading to nanofiber film.

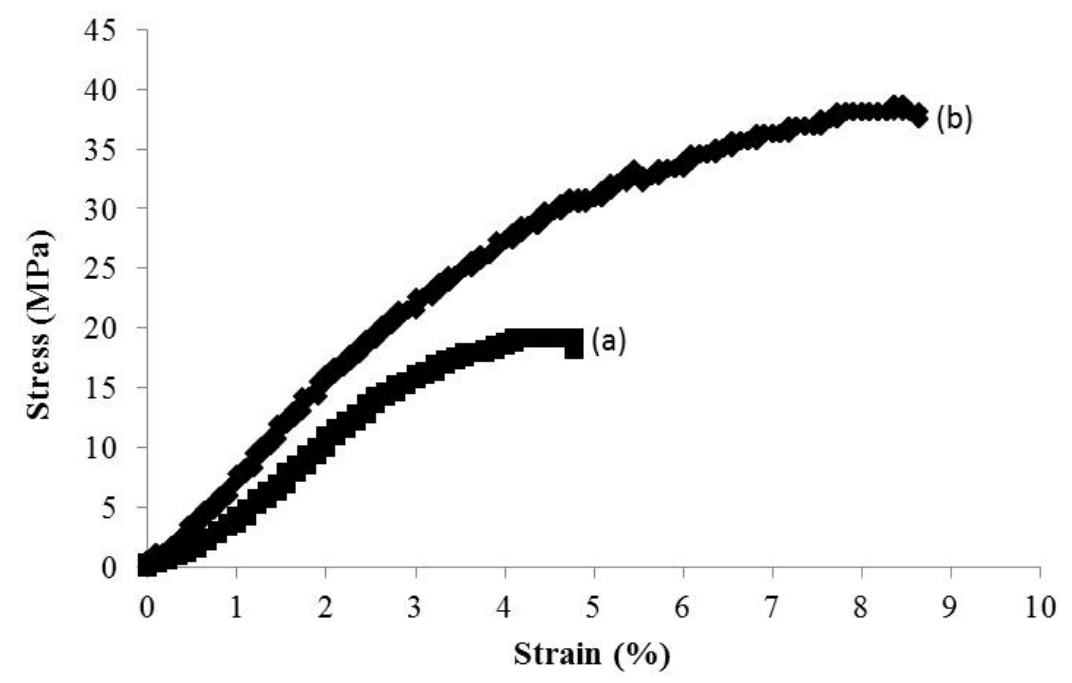

Figure 5. Stress-strain curves of films of 3 from (a) Avicel and (b) cotton after washing out alkali. 


\section{Conclusions}

This paper reports the formation of hierarchically self-assembled nanofiber films from amylose-grafted NaCMC films (3) with different DPs. The materials were synthesized by the chemoenzymatic method using NaCMCs from different sources, Avicel and cotton. The film with the clear nanofiber morphology was formed from the material with the higher DP (cotton) upon drying its aqueous alkaline solution, whereas the clear nanofiber morphology was not obtained in the film from the material with the lower DP (Avicel). By washing out the alkali from the films, the fibers merged at their interfacial areas. The obvious formation of the hierarchically self-assembled nanofibers in the film strengthened the mechanical properties under tensile mode.

Acknowledgments: A donation of phosphorylase by Ezaki Glico Co. Ltd., Osaka, Japan is gratefully acknowledged.

Conflicts of Interest: The authors declare no conflict of interest.

\section{References}

1. Klemm, D.; Heublein, B.; Fink, H.-P.; Bohn, A. Cellulose: Fascinating biopolymers and sustainable raw material. Angew. Chem. Int. Ed. 2005, 44, 3358-3393.

2. Klemm, D.; Kramer, F.; Moritz, S.; Lindström, T.; Ankerfors, M.; Gray, D.; Dorris, A. Nanocelluloses: A new family of nature-based materials. Angew. Chem. Int. Ed. 2011, 50, 5438-5466.

3. Beglou, M.J.; Haghi, A.K. Electrospun biodegdadable and biocompatible natural nanofibers: A detailed review. Cellul. Chem. Technol. 2008, 42, 441-462.

4. Isogai, A.; Saito, T.; Fukuzumi, H. TEMPO-oxidized cellulose nanofibers. Nanoscale 2011, 3, 71-85.

5. Abdul Khalil, H.P.S.; Bhat, A.H.; Ireana Yusra, A.F. Green composites from sustainable cellulose nanofibrils: A review. Carbohydr. Polym. 2012, 87, 963-979.

6. Saito, T.; Nishiyama, Y.; Putaux, J.L.; Vignon, M.; Isogai, A. Homogeneous suspensions of individualized microfibrils from TEMPO-catalyzed oxidation of native cellulose. Biomacromolecules 2006, 7, 1687-1691.

7. Saito, T.; Kimura, S.; Nishiyama, Y.; Isogai, A. Cellulose nanofibers prepared by TEMPO-mediated oxidation of native cellulose. Biomacromolecules 2007, 8, 2485-2491.

8. Abe, K.; Iwamoto, S.; Yano, H. Obtaining cellulose nanofibers with a uniform width of $15 \mathrm{~nm}$ from wood. Biomacromolecules 2007, 8, 3276-3278.

9. Kadokawa, J.; Arimura, T.; Takemoto, Y.; Yamamoto, K. Self-assembly of amylose-grafted carboxymethyl cellulose. Carbohydr. Polym. 2012, 90, 1371-1377.

10. Stephen, A.M.; Philips, G.O.; Williams, P.A. Food Polysaccharides and Their Applications; Taylor \& Francis: London, UK, 1995.

11. Matsuda, S.; Kaneko, Y.; Kadokawa, J. Chemoenzymatic synthesis of amylose-grafted chitosan. Macromol. Rapid Commun. 2007, 28, 863-867. 
12. Kaneko, Y.; Matsuda, S.; Kadokawa, J. Chemoenzymatic syntheses of amylose-grafted chitin and chitosan. Biomacromolecules 2007, 8, 3959-3964.

13. Omagari, Y.; Matsuda, S.; Kaneko, Y.; Kadokawa, J. Chemoenzymatic synthesis of amylose-grafted cellulose. Macromol. Biosci. 2009, 9, 450-455.

14. Kaneko, Y.; Kadokawa, J. Chemoenzymatic Synthesis of Amylose-Grafted Polymers. In Handbook of Carbohydrate Polymers: Development, Properties and Applications; Ito, R., Matsuo, Y., Eds.; Nova Science Publishers, Inc.: Hauppauge, NY, USA, 2009; Chapter 23; pp. 671-691.

15. Omagari, Y.; Kaneko, Y.; Kadokawa, J. Chemoenzymatic synthesis of amylose-grafted alginate and its formation of enzymatic disintegratable beads. Carbohydr. Polym. 2010, 82, 394-400.

16. Arimura, T.; Omagari, Y.; Yamamoto, K.; Kadokawa, J. Chemoenzymatic synthesis and hydrogelation of amylose-grafted xanthan gums. Int. J. Biol. Macromol. 2011, 49, 498-503.

17. Omagari, Y.; Kadokawa, J. Synthesis of heteropolysaccharides having amylose chains using phosphorylase-catalyzed enzymatic polymerization. Kobunshi Ronbunshu 2011, 68, 242-249.

18. Kadokawa, J. Synthesis of Amylose-Grafted Polysaccharide Materials by Phosphorylase-Catalyzed Enzymatic Polymerization. In Biobased Monomers, Polymers, and Materials; Smith, P.B., Gross, R.A., Eds.; ACS Symposium Series: Washington, DC, USA, 2012; Chapter 15; pp. 237-255.

19. Kadokawa, J.; Kaneko, Y. Engineering of Polysaccharide Materials-By PhosphorylaseCatalyzed Enzymatic Chain-Elongation; Pan Stanford Publishing Pte. Ltd.: Singapore, Singapore, 2013.

20. Ziegast, G.; Pfannemüller, B. Phosphorolytic syntheses with di-, oligo- and multi-functional primers. Carbohydr. Res. 1987, 160, 185-204.

21. Kitaoka, M.; Hayashi, K. Carbohydrate-processing phosphorolytic enzymes. Trends Glycosci. Glycotechnol. 2002, 14, 35-50.

22. Fujii, K.; Takata, H.; Yanase, M.; Terada, Y.; Ohdan, K.; Takaha, T.; Okada, S.; Kuriki, T. Bioengineering and application of novel glucose polymers. Biocatal. Biotransform. 2003, 21, 167-172.

23. Yanase, M.; Takaha, T.; Kuriki, T. $\alpha$-Glucan phosphorylase and its use in carbohydrate engineering. J. Food Agric. 2006, 86, 1631-1635.

24. Ohdan, K.; Fujii, K.; Yanase, M.; Takaha, T.; Kuriki, T. Enzymatic synthesis of amylose. Biocatal. Biotransform. 2006, 24, 77-81.

25. Seibel, J.; Jördening, H.-J.; Buchholz, K. Glycosylation with activated sugars using glycosyltransferases and transglycosidases. Biocatal. Biotranform. 2006, 24, 311-342.

26. Kobayashi, S.; Makino, A. Enzymatic polymer synthesis: An opportunity for green polymer chemistry. Chem. Rev. 2009, 109, 5288-5353.

27. Kadokawa, J.; Kobayashi, S. Polymer synthesis by enzymatic catalysis. Curr. Opin. Chem. Biol. 2010, 14, 145-153. 
28. Sasaki, M.; Sekiguchi, G.; Adschiri, T.; Arai, K. Rapid and selective conversion of cellulose to valuable chemical intermediates using supercritical water. In Proceedings of the 6 th International Symposium on Supercritical Fluids, 2003, Versailles, France, 28-30 April 2003; Volume 2, pp. 1417-1422.

29. Khullar, R.; Varshney, V.K.; Naithani, S.; Heinze, T.; Soni, P.L. Carboxymethylation of cellulosic material (average degree of polymerization 2600) isolated from cotton (Gossypium) linters with respect to degree of substitution and rheological behavior. J. Appl. Polym. Sci. 2005, 96, 1477-1482.

30. Olaru, N.; Olaru, L. Influence of organic diluents on cellulose carboxymethylation. Macromol. Chem. Phys. 2001, 202, 207-211.

31. Hosokawa, K.; Hanada, N.; Sato, S. Cationized carboxymethyl cellulose sodium salts with good chemical resistance and dispersibility. Japanese Patent 2002-201202. Filed 28 December 2000, Issued 19 July 2002.

32. Bhuiyan, S.H.; Rus'd, A.A.; Kitaoka, M.; Hayashi, K. Characterization of a hyperthermostable glycogen phosphorylase from Aquifex aeolicus expressed in Escherichia coli. J. Mol. Catal. B: Enzym. 2003, 22, 173-180.

33. Yanase, M.; Takata, H.; Fujii, K.; Takaha, T.; Kuriki, T. Cumulative effect of amino acid replacements results in enhanced thermostability of potato type $\mathrm{L} \alpha$-glucan phosphorylase. Appl. Environ. Microbiol. 2003, 71, 5433-5439.

34. Zobel, H.F. Starch crystal transformations and their industrial importance. Starch 1988, $40,1-7$. 


\title{
The Significance and Insignificance of Carbon Nanotube-Induced Inflammation
}

\author{
Matthew S.P. Boyles, Linda C. Stoehr, Paul Schlinkert, Martin Himly and \\ Albert Duschl
}

\begin{abstract}
In the present review article immune responses induced by carbon nanotubes (CNTs) are addressed. As inhalation is considered to be the primary entry route, and concern has been raised by similar high aspect ratio materials, the main focus lies on immune responses upon pulmonary exposure. Inflammation-related findings from both in vivo studies and in vitro models are reviewed, and the major responsible characteristics, which may drive CNT-induced inflammation in the lung, are discussed. In a second part, responses upon intentional administration of CNTs via subcutaneous and intravenous application are addressed, including their potential benefits and drawbacks for immunotherapy. Finally, the gastrointestinal tract as an alternative exposure route is briefly discussed. While there are many studies identifying numerous other factors involved in CNT-driven toxicity, e.g., cytotoxicity, oxidative stress, and genotoxicity, the focus of this review was kept solely on CNT-induced inflammation. Overall the literature has shown that CNTs are able to induce inflammation, which in some cases was a particularly robust response coinciding with the development of pro-fibrotic conditions. In the majority of cases the greatest inflammatory responses were associated with CNTs of considerable length and a high aspect ratio, accompanied by other factors like dispersion and sample purity.
\end{abstract}

Reprinted from Fibers. Cite as: Boyles, M.S.P.; Stoehr, L.C.; Schlinkert, P.; Himly, M.; Duschl, A. The Significance and Insignificance of Carbon NanotubeInduced Inflammation. Fibers 2014, 2, 45-74.

\section{Introduction}

According to The International Organization for Standardization (ISO), a carbon nanofiber (CNF) is defined as a (flexible or rigid) nano-object composed of carbon with two similar external dimensions in the nanoscale and the third dimension being significantly larger. Although this definition includes the group of carbon nanotubes (CNTs), classifying them as "hollow carbon nanofibers", many studies use the terms CNFs and CNTs to describe two distinct groups, with the difference being based on the orientation of the graphene sheet. Principally, CNTs can be either multi-walled (MWCNT) or single-walled (SWCNT), and are continuous rolls of graphene sheets that form tubes along a common axis, while CNFs are derived from different stacking arrangements of graphene layers; e.g., perpendicular to the fiber 
axis, inclined, parallel (unrolled) and even spiral-shaped [1]. As they are relatively easy to produce and their production costs are significantly lower compared to CNTs, there is a growing interest in using CNFs instead of CNTs for certain applications, mainly for electronics, reinforcing materials, and in the biomedical field [2,3]. Several studies suggest that these materials are especially promising for use in orthopaedic and dental applications [4-6]. Carbon nanotubes (CNTs), however, have already reached high volumes of industrial production, and with numerous commercial applications [7], are arguably the most notable high aspect ratio nanoparticle (HARN) currently on the market. Therefore, the primarily focus here is upon CNTs, while some attention is also given to CNFs.

With biological responses to CNTs being linked to the pathogenicity of asbestos, there is great concern surrounding CNT exposure, and there is a need to fully understand biological responses to this material. A key defense mechanism of the immune system and a factor highlighted in particle-induced responses is inflammation. In terms of the pathogenicity induced by asbestos, and other fibers and nanoparticles (NPs), the development of chronic inflammation [8] and progression of pulmonary fibrosis [9-11] are also often discussed. Therefore, using an assessment of CNT mediated inflammation it may be possible to predict and determine possible health implications associated with CNT exposure.

Inflammation attributed to pathogenicity induced by particles and fibers is often epitomized by the secretion of specific regulatory mediators, such as tumor necrosis factor-alpha (TNF- $\alpha$ ), interleukin (IL)-8, and monocyte chemoattractant protein (MCP)-1, by respiratory epithelial cells and alveolar macrophages [12]. These pro-inflammatory secreted proteins can be mediated by the activity of certain transcription factors and of intracellular signaling molecules, including nuclear factor-kappa B (NF-kB), activator protein-1 (AP-1), cyclic AMP (cAMP), and intracellular calcium, upon stimulation through oxidative and physical stress [13-15]. CNTs have also been shown to induce NF-KB and AP-1 activation, leading to oxidative stress-induced secretion of IL-8 [16-18]. In this review an insight into CNT-induced inflammatory responses is provided, with a main focus on pulmonary exposure, as inhalation is arguably the most relevant exposure route, through occupational exposure. In this case, inflammation-induced fibrosis is a key element to be considered. In addition to the pulmonary exposure route, subcutaneous, and intravenous administration, significant for nanomedical applications of CNTs, have gained increased attention and will, together with alternative exposure routes to CNTs, (namely the GIT), be discussed. 


\section{Immune Responses upon Pulmonary Exposure}

\subsection{Considerations for CNT Inhalation}

When assigning a similar pathogenicity to CNTs as has previously been observed in particularly aggressive materials, such as asbestos, there are two main issues to consider, biopersistence and respirability. The combination of both is essential for prolonged immune responses and for development of pulmonary fibrosis. With biopersistence, the clearance of inhaled material is hampered, through simple particle overload of immune cells, or potentially more importantly due to the physicochemical characteristics of the material. When fiber-like material, such as CNTs, have a length which exceeds $20 \mu \mathrm{m}$, a width under $3 \mu \mathrm{m}$ and are durable, becoming biopersistent, they are considered particularly pathogenic [19]. Normal clearance of material reaching the alveoli relies on alveolar macrophages, however, successful phagocytosis by these cells is limited to $20 \mu \mathrm{m}$ [20]. Fibers over this length can induce frustrated phagocytosis, which may lead to oxidative stress, enhanced cell proliferation, and chronic inflammation [21-23]. In Figure 1, frustrated phagocytosis can be observed in response to both (A) MWCNT and (B) long fiber amosite (LFA) asbestos. There have been some efforts to determine parameters for CNT biopersistence, such as deposition patterns and clearance rates.

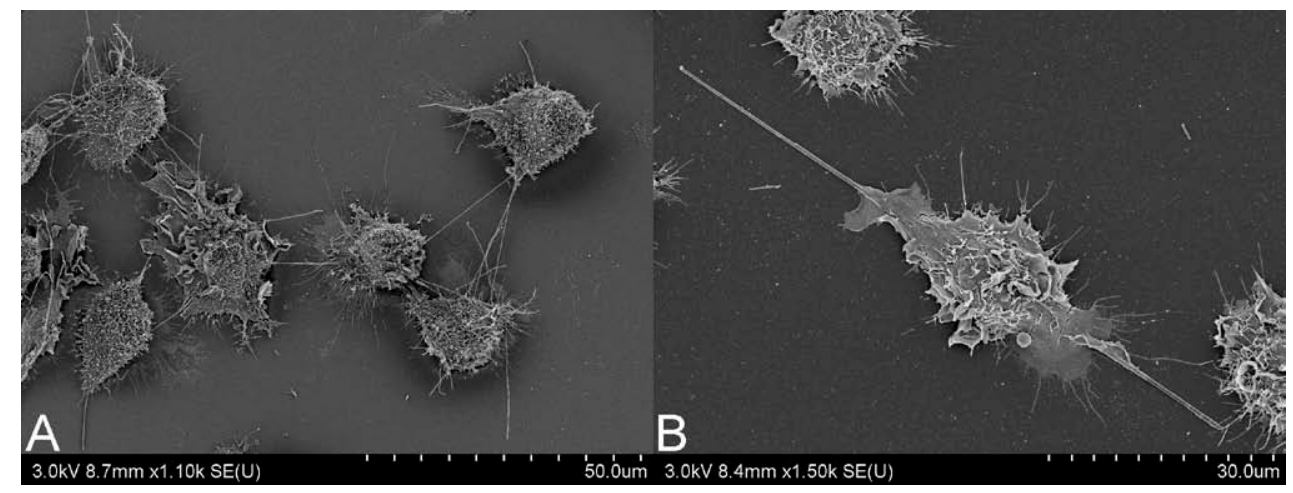

Figure 1. Scanning electron microscopy image of J774.A1 cells undergoing frustrated phagocytosis, induced by (A) multi-walled carbon nanotubes and (B) long fiber amosite asbestos. Scale bar is on bottom right of each image. Images courtesy of Matthew Boyles, Lesley Young and Vicki Stone.

Biopersistence. Recent literature has indicated that in some cases CNTs can be degraded by biological defensive mechanisms found within the lung. For example, the degradation of SWCNTs has been shown in the presence of hypochlorite, human neutrophil enzyme myeloperoxidase catalyze, and upon direct exposure to 
neutrophils and macrophages, with the degraded material eliciting no inflammatory reactions [24]. This result has been confirmed by the observation of impaired clearance of CNT in myeloperoxidase-deficient mice [25], and eosinophil peroxidase has also been shown to degrade SWCNT [26]. This is very promising and might indicate that the accumulation of certain CNTs within the lungs would not be so dramatic. However, neither neutrophils nor eosinophils are expected to be the normal responders to CNTs. The macrophages, which would be the cell type expected to clear irritating materials in the various compartments of the lung, have far less expression of the enzymes that have been shown to degrade CNT [24], urging care when dealing with questions of persistence. In particular, the accumulation of macrophages has often been associated with asbestos-related pathologies $[27,28]$. In addition, structurally, CNTs are diverse and findings on biological degradation cannot be considered to apply for all CNT materials. In a phagolysosome simulant fluid, Liu et al. [29] demonstrated similar findings to Kagan et al. [24] when assessing the durability of carboxylated SWCNT. While, adversely, when SWCNT received no functionalization, underwent high temperature annealing, reducing surface functional groups, or were functionalized by ozonolysis or aryl-sulfonation, no degradation was found [29], indicating a potential for biopersistence of these CNTs.

Respirability. The respirability of particularly CNTs, but also CNFs, is an important consideration. Due to electrostatic properties of CNTs and resultant formation of large agglomerates, it has been considered that CNTs are not respirable to distal lung regions, however, a number of studies have shown otherwise. Sprague Dawley rats exposed to vapor grown CNFs for 90 days in a subchronic inhalation study showed slight inflammatory effects (sub-acute to chronic) and changes in lung morphology for administered doses of $2.5-25 \mathrm{mg} / \mathrm{m}^{3}$, some of which were still persistent (although reduced) after a 30-day recovery period. The observed changes included thickening of the interstitial walls and hypertrophy/hyperplasia of type II alveolar cells [30]. A dose-dependent deposition of MWCNTs throughout the lungs of exposed mice (via inhalation) was found by Mitchell et al. [31]. Alveolar macrophages were found laden with CNTs. No pulmonary toxicity was observed, however, systemically there was reduced immune function, in the form of decreased natural killer (NK) cell function, which may indicate further translocation from the lungs [31]. Ryman-Rasmussen et al. [32] have also shown inhalation of MWCNTs $0.3-50 \mu \mathrm{m}$ in length. CNTs were observed throughout distal lung regions, within epithelial cells and alveolar macrophages. This study was conducted in naive and ovalbumin-sensitized mice. Combined, these treatments were shown to induce strong inflammatory responses with secretion of PDGF and MCP-1, and pro-fibrotic conditions with fibroblast proliferation, collagen deposition and secretion of IL-13, TGF- $\beta 1$, and IL-5. No fibrosis was observed with individual exposures, although inflammatory mediators were still present, with IL-13 and TGF- $\beta 1$ in 
response to ovalbumin, and PDGF and MCP-1 in response to MWCNTs. MWCNT inhalation of aggregates with an aerodynamic diameter of $<3 \mu \mathrm{m}$ also resulted in deposition within the alveolar region in a study conducted by Ma-Hock et al. [33]. Dose-dependent systemic and pulmonary inflammation was observed, as well as granuloma formation in the lungs and lymph nodes and the upper respiratory tract and immune cells were shown to accumulate. Uptake of MWCNTs was observed in both epithelial cells and alveolar macrophages with inhalation of $0.1,0.5$, or $2.5 \mathrm{mg} / \mathrm{m}^{3}$ MWCNTs. Although fibroblasts were present, no fibrosis was evident during the 13-week study. Systemic effects observed by Mitchell et al. [31], and Ma-Hock et al. [33] only infer the ability of MWCNT to translocate to neighboring tissues, while Ryman-Rasmussen et al. [34], and Mercer et al. [35,36] identified migration of CNTs to alveolar interstitium, subpleural tissue, and intrapleural spaces using inhalation or aspiration, respectively. As did Mercer et al. [37], with lung aspiration of relatively short $(3.86 \mu \mathrm{m})$ MWCNTs into C57BL/6J mice. In as little as seven days, no MWCNTs were found within the airways, instead a particle lung burden was observed within alveolar macrophages, alveolar tissue, granulomatous lesions and subpleural tissues. The presence of MWCNTs within alveolar tissue was associated with an increase in alveolar connective tissue thickness. With CNT inhalation, the translocation observed by Ryman-Rasmussen et al. [34] was associated with subpleural fibrogenesis. CNT translocation upon inhalation has been further confirmed in recent publications. When C57BL/6 J mice exposed to MWCNTs, with post-exposure periods of one day and almost one year, MWCNTs were found predominantly within the lymph nodes, but also within the diaphragm, chest wall, liver, kidney, heart and brain [38]. A study conducted under similar conditions highlighted inflammatory responses generated under these conditions, and the progression of fibrosis. During the 336-day post-exposure period the lung burden pattern indicated an early uptake within alveolar macrophages and gradual decline in this location, with an increasing burden within alveolar tissue as the 336 days progressed. This was accompanied by a rapid inflammatory response in the form on polymorphonuclear leukocyte (PMN) accumulation, which declined over time, and a time dependent deposition of collagen within the alveolar region [39]. In an acute inhalation study, Porter et al. [40] reported substantial MWCNT lung burden within the alveolar region, predominantly within macrophages, and a significant increase in PMNs, which was associated with mild fibrosis. Pleural penetration was also observed during this acute study. Each of these studies adds great weight to the similarities suggested for CNT-induced responses to those of asbestos.

Deposition. The deposition pattern of CNTs can be quite diverse, with subsequent variation to induced pulmonary responses. Deposition of CNTs throughout the pulmonary system is evident in many animal models, using administration by aspiration, inspiration, but also inhalation. With aerosolisation of MWCNT 
aggregates of aerodynamic diameter $714 \pm 328 \mathrm{~nm}$ Ryman-Rasmussen et al. [32] observed a homogenous distribution throughout the mouse lung, including alveoli, with particle aggregates and single MWCNTs within alveolar macrophages and epithelial cells. A size-dependent deposition was shown by Muller et al. [41] with intratracheal instillation of intact and ground MWCNT into rat lungs. Agglomerates of intact MWCNT (individual length $5.9 \mu \mathrm{m}$ ) would remain within the upper airways, while grinding to a length of $0.7 \mu \mathrm{m}$ increased dispersion throughout the lung tissue. Both samples were persistent within the lungs of rats for the duration of the study, however, the clearance of ground MWCNTs was faster than that of the pristine, with $81 \%$ remaining of the pristine after 60 days, and only $36 \%$ of the ground. Both were associated with an influx of neutrophils and eosinophils, granuloma formation, collagen deposition, and fibrogenesis. The inflammatory response was greater to the intact MWCNT, however, the granuloma formation to these CNTs was limited to the sites of CNT accumulation, mainly in the bronchi. The dispersed, ground CNTs induced lesions predominantly within alveolar spaces and interstitial tissue, and also stimulated an acute and prolonged TNF- $\alpha$ release, while TNF- $\alpha$ release in response to intact MWCNTs only appeared to be acute.

\subsection{CNT Instillation and Aspiration}

The use of these more common methods of in vivo pulmonary exposure to CNTs, but also direct injection into peritoneal and pleural cavities, provide valuable information regarding pulmonary responses to CNTs and CNFs, and determination of clearance mechanisms. An increase in immune cells and pro-inflammatory cytokines can be found in response to numerous CNT samples [22,35,41-43], and an induction of inflammation-related complications has often been shown, including granuloma formation, alveolar wall thickening [35,43-45], collagen deposition [46], and the development of fibrotic lesions [41,43].

An increase in alveolar macrophages, PMNs, pro-inflammatory cytokines and fibrosis, as well as oxidative stress, have been reported upon exposure of C57BL/6 mice to SWCNTs, CNFs, and asbestos via pharyngeal aspiration. The most severe effects and earliest onset were observed in treatments of SWCNTs, followed by CNFs, and then asbestos [47]. A significant neutrophil recruitment was also observed with 18-hour post-instillation of MWCNT into the rat lung, which was considered CNT length-dependent, as similar results were not evident with short or entangled samples [42]. An acute post-exposure period of six hours has also been shown to elicit significant immune responses to $100 \mu \mathrm{m}$ double-walled CNTs (DWCNTs). Certain pro-inflammatory markers, such as leptin and IL-6, were found elevated in the mouse lung, but not TNF- $\alpha$ or IL-1 [48]. In addition, in mice, an acute inflammatory response to SWCNT was found three hours after particle instillation, with measurements of 
MIP-2, MCP-1, and IL-6. This inflammatory response to SWCNT lasted throughout the 24-h exposure period [49].

The progress of fibrosis in response to CNT exposure is considered a response to prolonged inflammation and the development of pro-fibrotic conditions. The release of mediators such as PDGF and TGF- $\beta 1$, and the increase in proliferation and cell recruitment in response to CNT $[43,45,50]$ are considered to induce granuloma formation, alveolar wall thickening, and fibrosis [22,35,43-45]. The development of fibrosis may first rely on the resolution of the initial inflammatory response and removal of recruited immune cells [51], although the continued release of TNF- $\alpha$ and progression of fibrosis can coincide [41], and a fibrotic response can be found in the absence of any obvious acute inflammatory response, but in the presence of pro-fibrotic growth factors, such as platelet-derived growth factor $-\mathrm{A},-\mathrm{B}$, and $-\mathrm{C}[50,52]$. Additionally, the progression of fibrosis may occur during instances of CNT exposure to already inflamed environments, such as in allergic disorders. Such a synergistic response of CNTs was observed in ovalbumin-sensitized mice [32]. In a similar study intranasal exposure of CNTs and CNFs to ovalbumin-sensitized mice induced an increase in immune cells in both treatments, compared to ovalbumin alone [53]. Total cell counts, and those of neutrophils and macrophages were elevated in BAL fluid in response to CNFs, however, eosinophil influx was not observed, indicating a less significant allergic airway inflammation. In contrast, all these cell phenotypes were found increased in response to CNTs. Both carbon forms were also shown to promote allergen-specific IgE and IgG production; however, generally the effects of CNFs were less potent than for CNTs, presumably due to the higher aspect ratio and biopersistence of the latter.

With many exposure models using either CNT instillation or aspiration it is important to assess the differences between these methods and CNT inhalation. Using repeated inhalations or aspiration short SWCNTs $(<1 \mu \mathrm{m})$, with considerably high Fe content (17 wt\%), were shown to induce a strong inflammatory response, with influx of immune cells, increased pro-inflammatory cytokine release, and also the progression of fibrosis. However, rats exposed via inhalation were considered more susceptible than those exposed via aspiration [54]. 


\section{Pulmonary responses to CNTs}

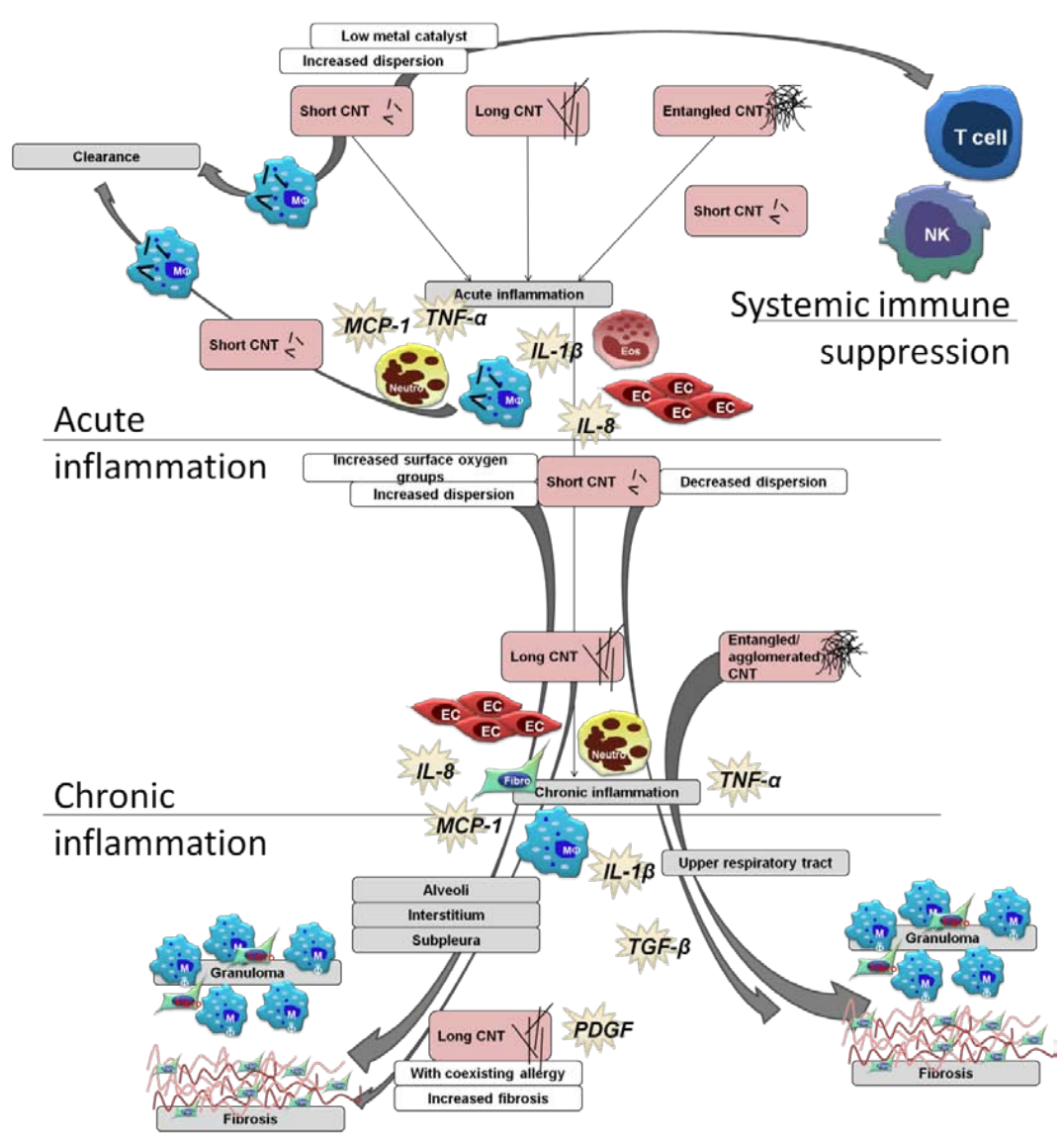

Fibrosis

Figure 2. Schematic view of pulmonary immune cell responses related to CNT characteristics, using a selection of the literature reviewed in this article. A range of responses can be seen, with the secretion of both pro-inflammatory and pro-fibrotic mediators, and effects upon epithelial cells (EC), T cells, monocytes (MO), macrophages, natural killer cells (NK), eosinophils (Eos), and neutrophils (neutro). Short, long, and entangled CNTs all are able to induce acute and chronic inflammation, and all able to induce granulomas and fibrosis. Fibrotic lesions induced by aggregated and entangled CNTs are restricted to the upper airways, while well-dispersed short and long CNTs are found within the alveoli and interstitium, with associated fibrosis. Allergen sensitization is shown to enhance inflammation and fibrosis induced by CNTs, as is CNT length. At times, a systemic immune effect can be observed without obvious pulmonary inflammation. [31,32,34,41,42,52,55]. 


\subsection{In vivo Determination of Responsible Characteristics and Significance of in Vitro Models}

There are many characteristics proposed that may contribute to CNT immunotoxicity and enhance pathogenicity, including metal contaminants and their bioavailability, high aspect ratio, aerodynamic diameter, as well as structural defects, pertaining to sample purity, surface chemistry, and sample dispersion. For hazard assessment and to allow safe usage and production it is important to establish which components are less or more influential, and possibly which biological environments are less or more susceptible. Here, we want to provide a few examples of how each characteristic has been shown to play a role in vivo; and furthermore, draw attention to in vitro systems which have attributed toxicity to these same CNT characteristics and, therefore, can be used as fast, high throughput methods to assess these characteristics. There is extensive literature that has elucidated upon which CNT characteristics are most responsible for the resultant toxicity, and to cover them all is beyond the scope of this review. To provide an overview of pulmonary responses to CNTs, Figure 2 offers a schematic view of immune cell responses related to CNT exposure characteristics.

Involvement of oxidative stress and metal impurities. Much of the particle toxicology performed in the past has placed an emphasis upon the induction of oxidative stress. This may also be relevant for CNT exposures, primarily due to the activity of metal contaminants found within many CNT samples, as the redox activity of metals, such as iron, is known to induce activation of key inflammatory instigators, such as NF- $\mathrm{kB}, \mathrm{AP}-1$, and p53 [56]. There are numerous studies that can shed some light on this subject. With exposure to material of fiber-like dimensions [57,58], including MWCNT [59], reactive oxygen species (ROS) production during phagocytosis is intensified; with associated inflammatory responses [21], through ROS-mediated NF- $\kappa B$ activation [16]. In vitro, this has been attributed to the initiation of respiratory burst $[21,59]$ and, at times, to fiber-induced frustrated phagocytosis [20,59]. When pro-inflammatory markers, such as leptin and IL-6, were found in vivo, in the absence of ROS, the lack of redox activity was attributed to either a low presence of metal contaminants or to the ability of relatively pure CNT samples to appropriate ROS [48].

Carbon structure, metal impurities and sample dispersion. An acute inflammatory response has been observed in vivo with exposure to iron-depleted SWCNTs with the systematic presentation of neutrophils, lymphocytes and macrophages, respectively, as the post-exposure period progressed. An early elevation of TNF- $\alpha$ and IL- $1 \beta$ was observed, at later stages progressing to elevated TGF- $\beta 1$, granuloma formation, collagen deposition, and fibrosis [43]. A parallel in vitro study was found to be both complementary and contrary. In RAW 264.7 macrophages the same SWCNTs did not induce the secretion of TNF- $\alpha$, nor IL-1 $\beta$. However, the purified (iron depleted) SWCNTs were found to induce TGF- $\beta 1$ after six-hour exposure to $100 \mu \mathrm{g} / \mathrm{mL}$ [43], 
confirming that the progression of a pro-fibrotic environment may be instigated by CNTs, and highlighting that iron content has a lesser importance in these responses. The above mentioned in vivo events were often associated with SWCNT aggregates, but observations were made of additional collagen deposition and alveolar wall thickening independent of SWCNT aggregates, and possibly due to undetectable, dispersed SWCNTs [43]. As previously mentioned, Muller et al. [41] identified inflammatory responses and patterns of fibrosis in vivo that were dependent of CNT dispersion within the lung. Ground MWCNTs provided a better dispersion, a greater capacity for reaching lower lung regions, and an enhanced inflammatory response when compared to intact MWCNT; this enhanced inflammatory response was corroborated in vitro with the exposure of peritoneal macrophages, when ground MWCNTs were shown to induce significant TNF- $\alpha$ expression whereas intact MWCNTs did not. This was later confirmed by Mercer et al. [35], with pharyngeal aspiration of well-dispersed SWCNTs similar to those used by Shvedova et al. [43]. With administration of the same SWCNT weight, a similar acute inflammatory response was observed in C57BL/ 6 mice; an acute influx of inflammatory cells was observed which subsided over time, and was not associated with the appearance of granulomatous lesions when SWCNTs were well-dispersed. SWCNTs were found within the interstitial spaces, in close proximity to the pleura, with the associated time-dependent alveolar wall thickening [35] that had also been observed by Shvedova et al. [43].

In a study controlling CNT properties such as metal contamination and oxygenated functional groups it was shown that biological responses can be influenced by CNT structural imperfections, and not metal contamination [60]. In vivo, immune cell influx, elevated IL-1 $\beta$, and TNF- $\alpha$ were all found to be enhanced in response to CNTs with high levels of surface oxygen groups, as was granuloma formation [60]. This dependency on structural defects was confirmed in vitro in the form of CNT-induced micronucleated binucleated cells [60], which provides a good corroboration between in vivo and in vitro systems. Although the endpoints differed, the causative factor was confirmed. Lam et al. [44] have also shown the significance of sample impurities, with an emphasis on metal contamination, with instillation of short CNTs. Substantial granuloma formation was observed irrespective of iron content, with considerable granuloma and lethality observed in exposure of mice to CNTs with high nickel content. Although it has been shown, by Kobayashi et al. [55], that short MWCNTs induce no marked inflammatory responses, it must be noted that the "low dose" used by Lam et al. [44] was considerably greater than the highest dose administered by Kobayashi et al. [55]. However, similar dosing with carbon black particles induced no such responses during the full exposure period used by Lam et al. [44]. When considering dose, in assessment of normal workplace exposure it may be prudent to remain reserved in respect to particle dosimetry. However, 
when considering an accident scenario exposure dose really has few limits. The main limiting factor may be respirability, and although Lam et al. [44] used instillation as their method of exposure the CNT dust samples were evaluated as a generated aerosol and were found to be predominantly of respirable size. In consideration of normal workplace exposure, it was reported by Maynard et al. [61] that with SWCNT dust handling there is potential exposure to $53 \mu \mathrm{g} / \mathrm{m}^{3}$, which would lead to $106.8 \mu \mathrm{g}$ SWCNT deposition upon lung epithelium per day. The dose used by Mercer et al. [35] of $10 \mu \mathrm{g}$ per mouse was a calculated representation of approximately 200 days normal workplace exposure in humans. With $0.5 \mathrm{mg}$ CNTs/ $\mathrm{kg}$ body weight, and the average body weight of a mouse being 20 grams, the dose used by Mercer et al. [35] lies within the dose range used by Kobayashi et al. [55] of 0.04, 0.2, and $1 \mathrm{mg} / \mathrm{kg}$ body weight of MWCNTs instilled into Sprague-Dawley rats; the dose used by Lam et al. [44] was certainly significantly higher at (again using average mouse weight of 20 grams) 5 and $25 \mathrm{mg} / \mathrm{kg}$ body weight, and can only be used in reference to an accident scenario, while the two studies by Mercer et al. [35] and Kobayashi et al. [55] provide a suitable determination of potential occupational exposure.

CNT length. An emerging pattern within current literature on in vivo and in vitro studies is the increased immunogenicity of particularly high aspect ratio CNTs, and, thus, it is tentatively proposed that this characteristic plays a pivotal role in inducing pulmonary inflammatory responses, and may be equally important in the proposed disease progression. The pathogenicity of any fibre can often be attributed to length and durability, both of which allow evasion of host abilities to remove fibres from distal lung regions. When a fiber is over 10-20 $\mu \mathrm{m}$ in length the ability of alveolar macrophages to remove these fibers is impaired, induced by frustrated phagocytosis, which allows for further interaction with epithelial cells and the opportunity for fibers to translocate to pleural spaces [20,62]. In previous fiber toxicology research, such studies looking into asbestos, length has been shown to be instrumental in inflammation-mediated disease progression. This is therefore a key aspect that has been considered for CNT hazard assessment.

MWCNT length- and shape-dependent responses have also been confirmed in vitro. In primary monocytes, Brown et al. [21] demonstrated that long, straight, well-dispersed CNTs can induce significant TNF- $\alpha$ release. The authors attributed these effects primarily to frustrated phagocytosis induced during these exposures, as responses to other material that were easily engulfed by phagocytes, such as short (a few microns) carbon nanofibers, small carbon nanofiber bundles and nanoparticle carbon black (NPCB), were not shown to be as strong, incidentally nor were responses to LFA [21]. This does raise a question to which other CNT components may play a role in CNT immunogenicity, as LFA would undoubtedly also cause frustrated phagocytosis. The role of oxidative stress was proposed in a later study by Brown et al. [63] when THP-1 cells were again found to secrete significant quantities 
of pro-inflammatory mediators, such as IL-1 $\beta$, in parallel with enhanced HO- 1 and Nrf2 gene expression in response to long straight MWCNT. These responses were not matched by short MWCNTs or small MWCNT bundles, neither by amorphous carbon particles or LFA [63]. LFA-induced frustrated phagocytosis was observed with exposure of J774A.1 murine macrophage cells in a study by Boyles et al. [59], which coincided with significant inflammation. However, the secretion of pro-inflammatory mediators in response to long straight MWCNTs was significantly higher than that of LFA-treated cells [59]. This study was designed to investigate many of the CNT properties proposed as potentially pathogenic: CNT length, iron content, and crystallinity. It was shown that long MWCNTs could induce considerable pro-inflammatory, pro-fibrotic, and pro-angiogenic conditions, with exposure of mouse and human phagocytes. MWCNTs of lengths greater than $30 \mu \mathrm{m}$ were shown to elicit significantly greater release of MCP-1, TGF- $\beta 1$, and TNF- $\alpha$, when compared to shorter MWCNTs $(<20 \mu \mathrm{m})$ but also to known pathogenic material, such as LFA. The iron content and crystal structure was found to have less impact on MWCNT immunogenicity [59]. A length-dependent immune response was further confirmed in A549 epithelial cells when, through oxidant dependent NF- $\mathrm{KB}$ activation, MWCNTs of up to $30 \mu \mathrm{m}$ in length were shown to induce significant IL-8 secretion, which was not found with exposure of carbon particles [16].

The conclusions of these in vitro studies complement numerous in vivo studies. Fibrogenic and inflammatory responses to MWCNTs of a particularly high aspect ratio have been shown within the lung [42,52] and within peritoneal and pleural cavities, often greater than those of shorter CNTs, other particles or known pathogenic fibers $[22,64]$. At times the exposure to short CNTs and small CNT agglomerates, in vivo, has induced little or no prolonged inflammation or fibrosis, and it appears that lung defense mechanisms can effectively control exposures of short MWCNTs [55]. Injection of high aspect ratio CNTs into the peritoneal cavity of mice was shown to induce an enhanced inflammatory response and encourage considerable granuloma formation, this was in comparison to negative controls but also when compared to short CNTs and long fiber amosite [22]. Long MWCNTs have been shown to induce a significant cell recruitment upon instillation into the rat lung, not observed in treatments of short nor entangled CNTs [42]. In a chronic exposure study, with post-exposure periods of three days to six months, intratracheal instillation of well-dispersed short MWCNTs $(<20 \mu \mathrm{m})$, Kobayashi et al. [55] observed no significant long-term inflammation within the rat lung. Neutrophil and eosinophil recruitment was observed during an acute period, which was not evident after chronic exposure, while it was maintained with treatments of crystalline silica. Observations of alveolar macrophages laden with MWCNTs and the lack of inflammation [55] indicated the ability of rats to safely control MWCNTs of this length. 


\subsection{The Role of in Vitro Systems}

The use of in vitro systems, of course, does not truly mimic the in vivo situation. However, they may be used as relatively fast and cheap assays for determining biological responses to CNTs, as well as to other nanomaterials (NMs), and can provide medium-to-high throughput screening methods for dealing with large sample numbers, e.g., for monitoring, or toxicity profiling. This method of in vitro toxicity profiling has recently been reported by Snyder-Talkington et al. [65] using the exposure of mice and small airway epithelial cells (SAEC) to MWCNTs, and subsequent correlation of MWCNT induced lung pathology data with tissue and SAEC mRNA expression. Many markers perceived as responsible for lung inflammation and fibrosis were identified during the analysis of tissue mRNA, and a good correlation of VEGF and CCL2 levels were shown in the coinciding in vitro study, confirming the usefulness of in vitro toxicity profiling. In vitro systems can also provide determination of acute pro-inflammatory responses, they can be used to infer the initiation of pro-fibrotic environments upon particle exposure, and to determine the initiation of fibrosis more directly. For example the collagen formation by fibroblast cells in vitro was shown in response to SWCNTs dispersed using both natural lung surfactant and acetone, but not in the absence of a dispersion aid; this was substantiated in vivo when the same materials were found to induce collagen deposition upon aspiration by C57BL/6J mice [46].

The studies presented in this section have demonstrated that the outcome of in vitro studies can complement those of in vivo systems. In vitro it must, however, be communicated that in vitro studies can be used as standalone investigations, particularly with the development of complex multi-cellular systems and, additionally, when addressing questions concerning cellular mechanisms, in vitro will often be the only appropriate method. A good example of this concerns the secretion of IL-1 $\beta$. Neither Boyles et al. [59], nor Hirano et al. [17] observed any secretion of IL-1 $\beta$, although Hirano et al. [17] reported the expression of IL-1 genes were enhanced, and both studies identified numerous other pro-inflammatory cytokine secretions. The induction of IL-1ßrelease in response to CNT exposure may be slightly more complicated than for most other cytokines and may be due to the nature of IL- $1 \beta$ formation, as the transcription, translation, and eventual release of mature IL-1 $\beta$ usually require more than one stimulus. For the initial expression and synthesis of pro-IL-1, TLR binding can provide the first stimulus. Formation of mature IL-1 $\beta$ occurs through the action of inflammasome-associated caspase-1 enzyme [66]. The involvement of inflammasomes in many inflammatory disorders is evident, in cases of allergic asthma [67], in relation to known carcinogens, such as silica, associated with fiber-mediated respiratory disorders such asbestosis [11], and also in inflammatory responses to CNTs [66]. DWCNTs have been shown to induce IL-1 maturation, attributed to NLRP3 inflammasome activation, which was thought 
to occur during binding and internalisation of CNTs when potassium was released through increased cell membrane permeability [66]. The ROS generated during CNT-induced membrane impairment, through frustrated phagocytosis, has also been implicated in inflammasome activation by Dostert et al. [11]. Palomäki et al. [68], who attributed NLRP3 inflammasome activation-induced IL-1 $\beta$ secretion to the fiber properties of long CNTs and asbestos. These studies highlight the importance of in vitro models, and incidentally, both place an importance on CNT aspect ratio in immune regulation.

The emerging field of in vitro co-culture techniques has been used for the assessment of numerous NMs, and is advancing in vitro methods towards a more realistic view of particle interactions and biological responses, as they allow for the complex interaction between phenotypically distinct cells. A comparison between a complex triple cell co-culture model, consisting of monocyte-derived macrophages (MDM), monocyte-derived dendritic cells (MDDC), and 16HBE14o- epithelial cells, with each of these cells grown under traditional single cell culture conditions, has shown no difference in CNT induced cytotoxicity or inflammation, when assessing the different cell culture conditions [69]. A finding also reported by Müller et al. [70], who also observed no differences between these systems when assessing the oxidative potential of CNTs. Here, Clift et al. [69] differ, as they did demonstrate a difference between culture methods for oxidative potential, which is in line with a study performed by Gasser et al. [71] who also determined that co-culture and mono-culture exposures provide different responses for oxidative stress, and interestingly also for immune responses. The development of these techniques has been well reported, however, as yet conclusions regarding whether these systems provide different responses to NMs are undetermined, they do, however, add another dimension to in vitro toxicity testing.

This review has, thus far, focused on CNT immunogenicity within the pulmonary system, inflammation-related disease progression and in vitro systems related to pulmonary exposures, as this is still considered the primary exposure route. However, numerous studies have been performed concerning alternative exposure routes, such as subcutaneous implantation and intravenous injection, or, ingestion. All of which warrant consideration, and in terms of subcutaneous implantation and intravenous injection, often relate to intentional administration with proposed nanomedical applications. These aspects will be covered in the subsequent sections.

\section{Intentional Administration of CNTs-Nanomedical Applications}

As CNTs have been shown to easily penetrate the plasma membrane [72] and enter cells, they are therefore proposed in promising biomedical applications, such as nanovehicles in drug delivery systems [73], and for the transport of proteins [74], peptides [75], and DNA [76] into cells. Moreover, CNTs may exert an excellent 
adjuvant function when used as a carrier in immunization protocols, which has been shown for macromolecules [77-79] as well as for peptides [80] and small haptens. This also bears the potential to use CNTs as adjuvants in the production of antibodies for medical treatment, or for the development of immunoassays for pesticides, drugs of abuse, environmental contaminants, food additives, hormones, and toxins [81]. Due to their hydrophobic and, often, water-insoluble nature, CNTs have been subjected to an array of functionalization with various compounds resulting in different biological effects [82]. However, water-dispersible CNT biodistribution studies in mice, using the intravenous administration, have revealed that CNTs localize primarily within the liver and lung, and to a lesser extent the kidney and spleen. This form of CNT was considered biocompatible as TNF- $\alpha$ expression in hepatocytes cultured with CNTs was comparable to those of controls [83].

As holds true for any NM, the potential use of CNTs in nanomedicine relies upon a balance between desirable and undesirable immune responses (Figure 3). This can be evaluated with observations of the immunotoxicological profile of systemically administered CNTs [84]. The list of desirable immune effects can include increased vaccine efficacy, or improved treatments of cancer, and various inflammatory, autoimmune and infectious disorders; both immune stimulation as well as immune suppression can be the desired outcome. The impact of CNTs on the balance between the desirable and undesirable effects in potential therapeutic applications is summarized in Figure 3. Adverse reactions to CNTs can comprise of immunostimulatory responses such as hypersensitivity reactions, anaphylaxis, coagulopathy, but also immunosuppression, lowering the body's response against infections with pathogens or against malignant cells [85]. Apart from direct immune activation, adverse effects may include hemolysis and platelet aggregation leading to coagulopathy, both of which cause hepato- and nephrotoxicity and, furthermore, secondary immune effects. Moderate platelet aggregation by CNT-driven GPIIb/IIIa activation has been observed in vitro using human platelets (independent of protein kinase C), which was corroborated in vivo by vascular thrombosis upon CNT infusion in rats [86]. The authors could not exclude CNT metal contamination as the source of this response. Depending on surface charge CNTs induce ROS, and, thus, activate platelets. Whereas bridging of platelets via their GPIIb/IIIa receptor appears reliant on CNT morphology, as spheres of fullerene did not display such effects. Meanwhile, platelet-aggregating activity in response to CNTs has been reported to act via a mechanism involving calcium influx in human thrombocytes [87]. 


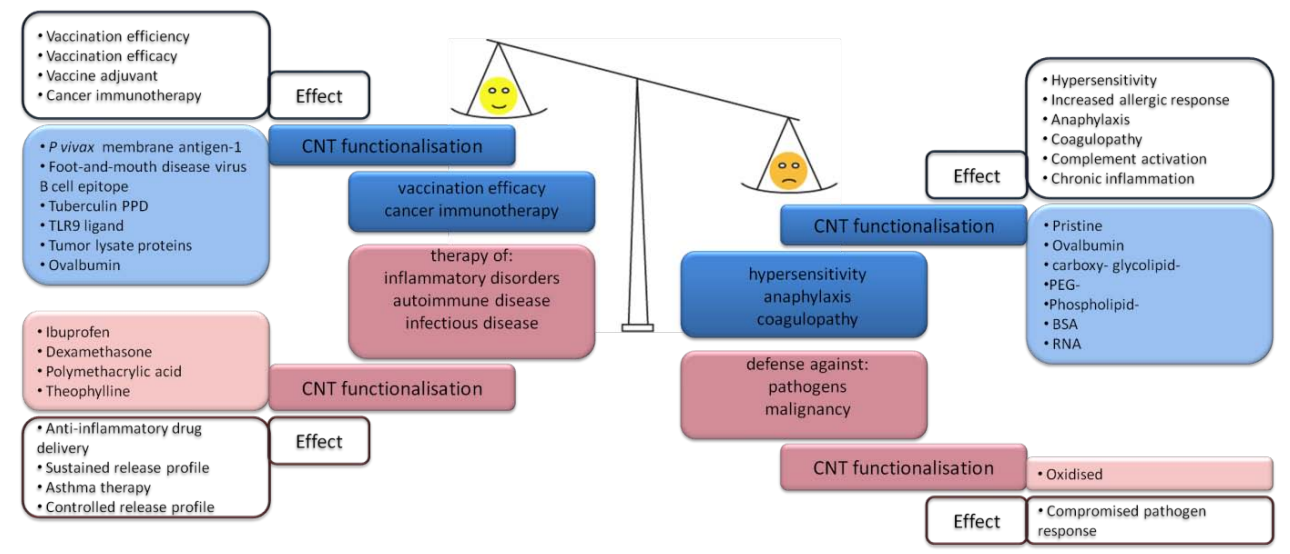

Figure 3. Desirable versus adverse effects may be due to immunostimulatory (blue) as well as immunosuppressive (red) functions of CNTs. In nanomedical applications of the desirable effects of intentionally administered CNT-based drugs have to outbalance adverse effects $[77,79,80,86,88-97]$.

\subsection{CNT-Induced Immune Responses upon Subcutaneous Administration}

Subcutaneous injection is a common and often the most effective way to administer drugs, as the tissue readily absorbs the administered substance due to its high vascularity. CNTs are being considered as drug delivery agents, and as such, administration of drugs carried by CNTs might affect the subcutaneous tissue homeostasis. The subcutaneous injection of MWCNTs has been shown in BALB/c mice to induce short-term immune responses. CNTs were observed within macrophages at the site of administration, accompanied by low levels of lymphocyte recruitment, collagen deposition and complement activation. Many inflammation-related cytokines were up-regulated during acute exposure to MWCNTs, including IL-17, IL-1 $\beta$, IFN- $\gamma$, IL-1 $\alpha$, IL-2, IL-3, IL-6, IL-10, IL-13, G-CSF, GM-CSF, and TNF-a; all of which subsided over time [98]. Furthermore, the subcutaneous injection of MWCNTs had previously been shown by Meng et al. [99] to induce similar cytokine release and macrophage and complement activation in tumor-bearing mice. With the exception of the lymph nodes, no obvious accumulation of the CNTs was found in liver, spleen, kidney, or heart. The group of Kazuyuki Tohji has focused several studies on the effects of CNTs on the subcutaneous tissue of rats. When hat-stacked carbon nanofibers (H-CNFs) were implanted into the subcutaneous layer of rats, macrophages were recruited and foreign body giant cells were formed at the affected tissues. Additionally, $\mathrm{H}-\mathrm{CNF}$ were found within the phagocyte lysosomal vacuoles [100]. In a more recent long-term exposure study, the same group examined biopersistence of powdered 
tangled oxidized MWCNTs (t-ox-MWCNTs) after implantation into the subcutaneous tissue of rats. Segments of the tissue were examined one week, one year and two years after implantation. At all three time points, large agglomerates had formed which were found in close proximity with fibroblasts and foreign body giant cells; smaller agglomerates were engulfed by phagocytes. With one and two years post-exposure periods, t-ox-MWCNTs were shown to migrate into the connective adipose tissue [101]. There are currently only few studies investigating the response of CNTs on subcutaneous tissue and additional research is needed in these areas if CNTs conjugates are to be used as nanomedicines in this fashion.

\subsection{CNT Induced Immune Responses within the Venous System-Pro-Inflammatory Effects}

Unspecific immune activation mechanisms by CNTs can operate via ROS production [102] or secretion of acute phase proteins like TNF- $\alpha$, IFN- $\gamma$, IL- 8 , IL-1 $\beta$, or IL- 6 from CNT-targeted cells [103]. The source of these pro-inflammatory molecules in case of systemically administered CNTs ranges from endothelial cells (upon vascular endothelium damage) to fibroblasts and immune cells within the blood and liver. There has been some debate in regard to whether the observed effects were due to the CNTs themselves or to present impurities, which also demands considerations of the pro-inflammatory impact due to coating procedures or composites [104,105]. Several studies emphasized the importance of CNT functionalization, where surface coating by polyethylene glycol (PEGylation) seemed to increase CNT-mediated activation of neutrophils and hence stimulate biodegradation [106]. While at high concentrations being cytotoxic, low doses of CNTs administered systemically mediated the NF-kB-dependent production of TNF- $\alpha$ and IFN- $\gamma$ in human lymphocytes, resulting in indirect toxicity [107].

MWCNTs functionalized with single-stranded DNA were administered intraperitoneally into rats and a pro-inflammatory profile was induced in the liver by a combination of oxidative stress and production of NF-kB-driven cytokines [108]. Using a proteomic approach, based on isobaric tagged relative and absolute quantification (iTRAQ)-coupled 2D liquid chromatography-tandem mass spectrometry, 30 differentially expressed proteins involved in cell cycle arrest, DNA repair, stress-induced apoptosis, as well as NF- $\mathrm{kB}-$ driven inflammation were found in human HepG2 liver cells upon exposure to oxidized SWCNTs [109]. For the investigation of genotoxicity and pro-inflammatory responses in the liver, the eventual terminus of CNTs with intravenous, intraperitoneal, or subcutaneous administration, but also when inhaled or ingested, the hepatoblastoma cell line C3A was proposed as a well-suited model [110], and just a very limited pro-inflammatory response to CNTs was observed, including a dose dependent increase in IL-8 secretion [111]. 
As previously discussed, induction of an innate immune response can be driven by various NM via the activation of the NLRP3 inflammasome, and this may even represent a desired effect for optimising the efficacy of a vaccine [112]. Nanomaterial-driven activation of innate immune responses includes specific interaction of NM with a large array of pattern-recognition receptors including Toll-like receptors (TLRs), C-type lectine receptors (CLRs), and scavenger receptors expressed on the plasma membrane, or different classes of NOD-like receptors (NLRs) and RIG-I-like receptors inside the cell [113]. In one study, the surface chemistry of CNTs was changed in order to target them rather to scavenger receptors and to a lesser extent to the usually preferred mannose receptors followed by uptake. This was shown to reduce NF-kB-mediated immunotoxicity in vitro in THP-1 human monocytic cells, as well as in vivo [114].

Upon systemic administration of NM, including CNTs, the complement system also represents an important candidate for innate immune activation $[115,116]$. The importance of CNT functionalization in regard to complement activation has been reviewed extensively [94,117]. In addition to the classical pathway via $\mathrm{C} 1$ and $\mathrm{C}$-reactive protein (CRP), the involvement of the mannose-binding lectin (MBL)/ficolin pathway and the alternative pathway via C3 has been discussed for macrophage uptake and activation in dependent on the type of CNT functionalization. Excessive or inappropriate complement activation can cause tissue damage and autoimmune disease [118]. During complement activation the anaphylatoxins $\mathrm{C} 3 \mathrm{a}, \mathrm{C} 4 \mathrm{a}$, and the most potent $\mathrm{C} 5 \mathrm{a}$, accumulate, which are responsible for smooth muscle contractions (bronchospasm), histamine release from mast cells and enhanced vascular permeability, and, as such, represent typical signs of severe anaphylaxis. Complement activation in vitro associated with binding of $\mathrm{C} 1$ to $\mathrm{CNTs}$ was found to be impaired by protein adsorption in vivo [119]; and, also in vitro, coating of SWCNT with human serum albumin has been shown to induce complement activation through C1q-mediated classical and alternative pathways, while amphiphile functionalization of the SWCNTs, resulted in complement activation of the lectin pathway [120]. The impact of these findings in vivo still remains to be clarified. Selective protein binding in serum was found in psychosine-functionalized CNTs. A novel glycolipid coating was shown to mediate stable suspensions in biological fluids, however, complement activation via $\mathrm{C} 1$ and CRP was also reported [94,121].

\subsection{CNT-Induced Immune Responses within the Venous System-Immunomodulatory Profile}

The specific, adaptive immune mechanisms rely on highly variable receptors such as the major histocompatibility complex and the $\mathrm{T}$ cell receptor, as well as on antibodies. All have the capacity to recognize a large array of non-self compounds 
including engineered NM. In particular, the functionalization of CNTs with peptides and proteins, which represents a major playground for nanomedicine applications, bears a strong potential to activate adaptive immunity. Therefore, adverse reactions against CNTs themselves have to be distinguished from functionalization-derived immune effects. As such, dendritic cell (DC) activation resulting in a marked T helper (Th)1- and Th17-polarizing capability was achieved by functionalizing CNTs with a TLR-7 agonist [122]. Notably, the same concentration of TLR7 agonist alone or even in combination with CNT, but not covalently linked, did not reach the same effect underlining the potential of CNTs to be used as immunogenic carriers [123]. Immunomodulatory effects may, however, also arise from the spontaneous formation of a protein corona (PC) [124,125], which holds true also for other NMs. Recently, hydrogen bonds and charged interactions have been shown, by label-free mass spectrometry-based proteomics, to be the main driving forces for PC formation of unmodified, carboxylated, and polyvinylpyrrolidone-coated SW- and MWCNTs under cell culture conditions [126]. The authors concluded a low degree of selectivity for PC formation of CNTs, as no relationship between the protein corona composition and isoelectric point, aliphatic index or hydropathy was found. Selective PC formation would be attributed to differences in the amino acid content of the formed PC, and, hence, hydrophobic interactions and pi-stacking of the different involved proteins would represent the main driving forces. However, the pathophysiology of systemically administered NM may still be affected by various kinetic impacts during human plasma PC formation and two scenarios are possible: pristine toxic NM may be encapsulated within a protective bio-shell or pristine non-toxic NM become toxic due to PC formation [127]. Thus, selective cytotoxicity of CNTs may have an impact on immune deviation, as DCs, T cells, or B cells may be targeted specifically and an alteration in subsets of these cellular players may cause severe effects, in an immunological context. A pivotal role of MWCNTs was reported recently from healthy and mite-allergic patients, as CNTs were found to promote and suppress immune effects depending on which type of peripheral blood mononuclear cells they interacted with [128].

To date, there is still very limited information available on DC-targeting effects exerted by CNTs. Using mouse bone marrow-derived DCs, CNTs were identified as not specifically affecting antigen-presenting cells in comparison to other NM, such as $\mathrm{ZnO}$ [129]. However, recently CNTs have been shown in vitro to induce a lower immunogenic profile of human differentiated and activated DCs, based on transcriptional analysis [130]. Another study reported apoptosis of $\mathrm{T}$ cells using the Jurkat cell line and human peripheral blood-derived $\mathrm{T}$ cells, however, dispersion, aggregation, or high effective concentration may have caused the observed cytotoxicity [95]. At low concentrations no T cell receptor-dependent effect could be detected using pristine CNTs [131]. In mice, immunosuppression by 
acid-functionalized CNTs was observed in vivo and in vitro, and these effects were shown to be mediated by down-regulation of the cytotoxic $T$ cell response [132].

Direct binding of pristine CNTs to an antibody generated against fullerene has been described, so cross-reactivity seems to be also an issue for engineered NM [133]. However, even at very high concentrations no CNT-specific antibodies could be determined upon application of hapten carrier CNTs in mice [134]. In general, functionalized CNTs were reported as non-cytotoxic for primary B and T lymphocytes. Moreover, up-regulation of $\mathrm{B}$ and $\mathrm{T}$ cell-specific activation markers was not observed, while with less water-soluble functionalization a secretion of pro-inflammatory cytokines from human macrophages, NK cells and monocytes was present [135-137]. In contrast, in mice a decrease of NK cell function upon inhalation of MWCNTs had been observed, as described above [31]. A new term of "monocyte-activating CNTs" has been coined for a panel of oxidized MWCNTs for responses induced in THP-1 (monocytic) cells that were not shown in Jurkat ( $\mathrm{T}$ cells) [138]. As far as it can be presently stated, adaptive immunity thus seems to play a minor role in CNT-mediated immune effects, which contrasts the above-described situation in innate immunity, where CNTs have been shown to mediate considerate immunogenicity. However, the close interconnection between innate and adaptive immunity promises potential applications of CNTs as (Th1-promoting) immunostimulatory carriers (adjuvants) for vaccines [79,89,139], while on the other hand it poses the risk of side-effects, such as hypersensitivity [93].

In summary, it has been extensively shown that intentionally administered CNTs interact with the immune system, and this can have desirable or adverse effects. Physical parameters including length, purity, surface chemistry, and aggregation state represent decisive factors for CNT-mediated immunotoxicity. For the potential use as drug carriers or vaccines it is evident that CNTs should be synthesized with the following properties: short, functionalized, water-soluble or easily dispersible, and biodegradable.

\section{Other Routes of Exposure-Gastrointestinal Tract}

There is a clear potential risk of occupational exposure to CNTs. Therefore the focus of this review, and much of the current literature, has been the pulmonary system; and with the proposed use of CNTs in nanomedicines, intentionally administered CNTs, via subcutaneous and intravenous routes, have also been deemed prominent exposure routes. However, with an estimated surface area of $200 \mathrm{~m}^{2}$, the mucosal barrier of the gastrointestinal tract (GIT) provides a large exposure area for NMs [140] and is also one of the main routes for many NMs to enter the human body. In general, possible ingestion routes for nanoparticles include uptake via food (intentionally added, leaked from NM-containing food packaging or kitchen utensils, contamination of water, animals or plants after distribution of NM 
in the environment) or secondary ingestion following inhalation due to mucociliary clearance of inhaled particles. Applications of CNTs, which may allow ingestion, are steadily increasing, and can include their implementation as adsorbents in water purification systems [141], or as antimicrobial agents or stabilizing materials in food packaging [142]. Recently, Prajapati et al. [143] investigated the use of CNTs as carriers for orally administered anti-parasite treatment in rats, with possible application in humans, and Kou et al. [144] identified functionalized MWCNTs as a promising coating material of dental implants for better osseointegration [144]. There have been observations that residual hydrophobic compounds, which are often adsorbed to CNTs during their synthesis, were released more rapidly in the presence of gastric enzymes and increased salt concentration in simulated GI fluids. This would make these compounds more accessible and possibly more toxic upon ingestion [145], and with proposed applications that could easily lead to the exposure of CNTs to the GIT, there is a clear need for research into this exposure route. To the best of our knowledge, there are only a few studies that have examined possible adverse effects of CNTs on the GIT, of which few have addressed immunogenicity. The genotoxicity of CNTs has been investigated by Szendi and Varga [146] and Folkmann et al. [147]; oxidative stress-induced DNA damage was found in the liver of mice when CNTs were transported there after entry to the bloodstream via the GIT [147]. With administration of CNTs via oral gavage Lim et al. [148], and Philbrook et al. [149] have investigated foetal development. In response to 14 days exposure of MWCNTs to pregnant rats, no clear responses were observed, excluding a particularly high dose $(1000 \mathrm{mg} / \mathrm{kg} /$ day $)$ inducing an increase in thymus size. Additionally, no abnormal foetal developments occurred, implicating that MWCNTs were unable to cross the blood-placenta barrier [148]. However, Philbrook et al. [149] reported an increase in foetal skeletal and ocular anomalies when mice were exposed to $10 \mathrm{mg} / \mathrm{kg}$ of SWCNTs (1-2 nm diameter) after nine days. An increase in dose, to $100 \mathrm{mg} / \mathrm{kg}$, was not shown to affect any markers of foetal development [149]. The authors proposed that this was due to an increase in agglomeration with an increase in CNT concentration, resulting in a reduced adsorption rate by the GIT. Also using repeated oral gavage of both SW- and MWCNTs, Matsumoto et al. [150] found no obvious toxicological responses, including signs of inflammation with differential leukocyte counts, and also concluded that it was large CNTs agglomerates that reached the GIT which, due to their size, were not absorbed. If, however, CNTs were to be absorbed by the GIT, as postulated by Philbrook et al. [149] in respect to small single CNTs, their accumulation within the liver may be expected. The effects of CNTs upon ingestion are clearly an avenue that critically needs further investigation. 


\section{Conclusions}

The reported and proposed use of CNTs clearly demonstrates the potential and the worth of this material. With a high tensile strength, optical and semi-conductive properties, and easy functionalization CNTs can be applied within many industrial and medical fields. With an increased commercial focus on CNTs hazard assessment was inevitable and soon the biological response to CNTs was being likened to that of asbestos. This has mainly focused on assumptions of inhalation within the workplace, where it was proposed that particularly high aspect ratio CNTs would follow the toxicity pattern that is outlined in the "fiber paradigm", where fiber pathogenicity is associated with biopersistence, mainly governed by durability, length and diameter. Hence, CNTs have been shown to induce a state of frustrated phagocytosis in phagocytes in vitro, but also in recruited immune cells in vivo. If durable, CNTs would remain at the site of inflammation, causing the progression of chronic inflammatory disorders, such as fibrosis; which had been a key marker in asbestos-induced pathology. This attributed factor in CNT immunogenicity has been repeatedly reported, while other characteristics suggested to play a role in nanoparticle and pathogenic fiber immunogenicity, such as metal contamination, sample dispersion and structural defects, have been reported with inconsistent outcomes.

The area of intravenous administration is relatively well represented in CNT immunogenicity studies, and as with subcutaneous injection, intravenous injection is often focused on CNTs with a medical application. Here, it has been shown that CNTs can elicit varied responses; with extensive distribution, functionalized CNTs are shown to induce both low and high levels of inflammation, at times accompanied by other innate immune responses such as complement activation. It is often only the innate immune system that is affected, which is particularly relevant e.g., in vaccination. However, $\mathrm{f}-\mathrm{CNTs}$ have been shown to stimulate $\mathrm{T}$ cell responses through the activation of DCs and can, thus, affect adaptive immunity as well. Physical parameters including length, purity, surface characteristics, and aggregation state represent decisive factors for CNT-mediated immunotoxicity systemically, as has been shown within the pulmonary system. For a potential use as drug carriers or vaccines, short, functionalized, water-soluble or easily dispersible, and biodegradable CNTs may appear the most appropriate option for future nanomedical research. With the promising use of CNTs in methods of immunotherapy it will be important to determine when these immune responses are induced by the intended CNT functional group, and when it is CNT themselves initiating these responses, particularly when CNTs have been shown to enhance existing allergic conditions. It is clear that intentionally administered CNTs can interact with the immune system. More research is required for a better understanding of these complex interactions, to provide suitable risk-to-benefit assessment and to allow the safe use of CNTs with nanomedical applications. 
The unspecific innate immune responses to CNTs, which have been shown to progress to the establishment of pro-fibrotic environments within the lungs in many animal models, with specific inflammatory mediators also identified in vitro, has not been observed to the same degree upon subcutaneous injection or with GIT exposure. These two exposure routes, however, are areas of CNT immunogenicity that are severely lacking, simply in volume of research studies that have been conducted. This is a gap that should be filled, as immunotherapy may often use the subcutaneous route, and there are considerable instances of how CNTs may end up within the GIT and continue to the venous system and the liver.

In terms of CNFs, a general observation is that CNFs do show a tendency towards immune reactivity, but however, there are many inconsistencies in the studies available which make it hard to evaluate the findings appropriately. It is also not clear whether CNFs are less potent or more potent than CNTs. Some studies suggest CNFs to be less reactive due to their smaller surface compared to CNTs, as well as their smaller aspect ratios. However, agglomeration of CNTs was often observed, which again changes the specific surface areas to smaller values (effective surface area). Further research is necessary to fully understand the mechanisms that control the responses of the immune system to CNTs and CNFs. There have been clear characteristics identified as detrimental, therefore, to reduce the potential hazard of this material these classifications should be observed in future developments of applications and production techniques, to allow a reduced risk to those who may be exposed to CNT material.

Note added in proof: An extensive review series edited by Ali-Boucetta, Bussy and Kostarelos (Advanced Drug Delivery Reviews (Volume 65, Issue 15, Pages 1897-2134-December 2013)) concerns issues of safety and toxicity of CNTs in biology and medicine, also with implications for therapy and diagnostics, and covers numerous issues dealt within the present review in extensive detail.

Acknowledgments: This work was supported by the EU 7th framework program, Marie Curie Actions, Network for Initial Training NanoTOES (PITN-GA-2010-264506), the NanoValid project-Development of reference methods for hazard identification, risk assessment and LCA of engineered nanomaterials (grant agreement No: 263147), and by the Research Cluster Immunity in Cancer and Allergy (ICA) at PLUS.

Conflicts of Interest: The authors declare no conflict of interest.

\section{References}

1. Martin-Gullon, I.; Vera, J.; Conesa, J.A.; Gonzalez, J.L.; Merino, C. Differences between carbon nanofibers produced using $\mathrm{Fe}$ and $\mathrm{Ni}$ catalysts in a floating catalyst reactor. Carbon 2006, 44, 1572-1580. 
2. Kisin, E.R.; Murray, A.R.; Sargent, L.; Lowry, D.; Chirila, M.; Siegrist, K.J.; Schwegler-Berry, D.; Leonard, S.; Castranova, V.; Fadeel, B.; et al. Genotoxicity of carbon nanofibers: Are they potentially more or less dangerous than carbon nanotubes or asbestos? Toxicol. Appl. Pharmacol. 2011, 252, 1-10.

3. Sanchez, V.C.; Pietruska, J.R.; Miselis, N.R.; Hurt, R.H.; Kane, A.B. Biopersistence and potential adverse health impacts of fibrous nanomaterials: What have we learned from asbestos? Wiley Interdiscip. Rev. Nanomed. Nanobiotechnol. 2009, 1, 511-529.

4. Price, R.L.; Waid, M.C.; Haberstroh, K.M.; Webster, T.J. Selective bone cell adhesion on formulations containing carbon nanofibers. Biomaterials 2003, 24, 1877-1887.

5. Price, R.L.; Haberstroh, K.M.; Webster, T.J. Improved osteoblast viability in the presence of smaller nanometre dimensioned carbon fibres. Nanotechnology 2004, 15, 892-900.

6. Elias, K.L.; Price, R.L.; Webster, T.J. Enhanced functions of osteoblasts on nanometer diameter carbon fibers. Biomaterials 2002, 23, 3279-3287.

7. Tran, C.L.; Hankin, S.M.; Ross, B.; Aitken, R.J.; Jones, A.D.; Donaldson, K.; Stone, V.; Trantra, R. An Outline Scoping Study to Determine whether High Aspect Ratio Nanoparticles (HARN) should Raise the Same Concerns as do Asbestos Fibres. Report on Project CB0406 2008. Available online: http://www.safenano.org/Portals/3/SN_ Content/Documents/HARN.pdf (accessed on 1 November 2013).

8. Coker, R.K.; Laurent, G.J. Pulmonary fibrosis: Cytokines in the balance. Eur. Respir. J. 1998, 11, 1218-1221.

9. Mossman, B.T.; Churg, A. Mechanisms in the pathogenesis of asbestosis and silicosis. Am. J. Respir. Crit. Care Med. 1998, 157, 1666-1680.

10. Cassel, S.L.; Eisenbarth, S.C.; Iyer, S.S.; Sadler, J.J.; Colegio, O.R.; Tephly, L.A.; Carter, A.B.; Rothman, P.B.; Flavell, R.A.; Sutterwala, F.S. The Nalp3 inflammasome is essential for the development of silicosis. Proc. Natl. Acad. Sci. USA 2008, 105, 9035-9040.

11. Dostert, C.; Petrilli, V.; Van Bruggen, R.; Steele, C.; Mossman, B.T.; Tschopp, J. Innate immune activation through Nalp3 inflammasome sensing of asbestos and silica. Science 2008, 320, 674-677.

12. Driscoll, K.E.; Carter, J.; Hassenbein, D.; Howard, B. Cytokines and particle-induced inflammatory cell recruitment. Environ. Health Perspect. 1997, 105, 1159-1164.

13. Brown, D.M.; Hutchison, L.; Donaldson, K.; MacKenzie, S.J.; Dick, C.A.J.; Stone, V. The effect of oxidative stress on macrophages and lung epithelial cells: The role of phosphodiesterases 1 and 4. Toxicol. Lett. 2007, 168, 1-6.

14. Sen, C.K.; Roy, S.; Packer, L. Involvement of intracellular Ca2+ in oxidant-induced NF-[kappa]B activation. FEBS Lett. 1996, 385, 58-62.

15. Kannan, K.; Jain, S.K. Oxidative stress and apoptosis. Pathophysiology 2000, 7, 153-163.

16. Han, M.; Ye, S.; Wen, W.; Zhang, Q. Oxidative stress-mediated pro-inflammatory responses in lung epithelial cells exposed to multi-walled carbon nanotubes. In Proceedings of the 2010 4th International Conference on Bioinformatics and Biomedical Engineering (iCBBE), Chengdu, China, 18-20 June 2010; pp. 1-4. 
17. Hirano, S.; Fujitani, Y.; Furuyama, A.; Kanno, S. Uptake and cytotoxic effects of multi-walled carbon nanotubes in human bronchial epithelial cells. Toxicol. Appl. Pharmacol. 2010, 249, 8-15.

18. Pacurari, M.; Yin, X.J.; Zhao, J.; Ding, M.; Leonard, S.S.; Schwegler-Berry, D.; Ducatman, B.S.; Sbarra, D.; Hoover, M.D.; Castranova, V.; Vallyathan, V. Raw single-wall carbon nanotubes induce oxidative stress and activate MAPKs, AP-1, NF-kappaB, and Akt in normal and malignant human mesothelial cells. Environ. Health Perspect. 2008, 116, 1211-1217.

19. Donaldson, K.; Tran, C.L. An introduction to the short-term toxicology of respirable industrial fibres. Mutat. Res. Fundam. Mol. Mech. Mutagen. 2004, 553, 5-9.

20. Dörger, M.; Münzing, S.; Allmeling, A.-M.; Messmer, K.; Krombach, F. Differential responses of rat alveolar and peritoneal macrophages to man-made vitreous fibers in vitro. Environ. Res. 2001, 85, 207-214.

21. Brown, D.M.; Kinloch, I.A.; Bangert, U.; Windle, A.H.; Walter, D.M.; Walker, G.S.; Scotchford, C.A.; Donaldson, K.; Stone, V. An in vitro study of the potential of carbon nanotubes and nanofibres to induce inflammatory mediators and frustrated phagocytosis. Carbon 2007, 45, 1743-1756.

22. Poland, C.A.; Duffin, R.; Kinloch, I.; Maynard, A.; Wallace, W.A.H.; Seaton, A.; Stone, V.; Brown, S.; MacNee, W.; Donaldson, K. Carbon nanotubes introduced into the abdominal cavity of mice show asbestos-like pathogenicity in a pilot study. Nat. Nanotechnol. 2008, 3, 423-428.

23. Ye, J.; Shi, X.; Jones, W.; Rojanasakul, Y.; Cheng, N.; Schwegler-Berry, D.; Baron, P.; Deye, G.J.; Li, C.; Castranova, V. Critical role of glass fiber length in TNF-alpha production and transcription factor activation in macrophages. Am. J. Physiol. 1999, 276, L426-L434.

24. Kagan, V.E.; Konduru, N.V.; Feng, W.; Allen, B.L.; Conroy, J.; Volkov, Y.; Vlasova, I.I.; Belikova, N.A.; Yanamala, N.; Kapralov, A.; et al. Carbon nanotubes degraded by neutrophil myeloperoxidase induce less pulmonary inflammation. Nat. Nanotechnol. 2010, 5, 354-359.

25. Shvedova, A.A.; Kapralov, A.A.; Feng, W.H.; Kisin, E.R.; Murray, A.R.; Mercer, R.R.; St. Croix, C.M.; Lang, M.A.; Watkins, S.C.; Konduru, N.V.; et al. Impaired clearance and enhanced pulmonary inflammatory/fibrotic response to carbon nanotubes in myeloperoxidase-deficient mice. PLoS One 2012, 7, e30923.

26. Andón, F.T.; Kapralov, A.A.; Yanamala, N.; Feng, W.; Baygan, A.; Chambers, B.J.; Hultenby, K.; Ye, F.; Toprak, M.S.; Brandner, B.D.; et al. Biodegradation of single-walled carbon nanotubes by eosinophil peroxidase. Small 2013, 9, 2721-2729.

27. Spurzem, J.R.; Saltini, C.; Rom, W.; Winchester, R.J.; Crystal, R.G. Mechanisms of macrophage accumulation in the lungs of asbestos-exposed subjects. Am. Rev. Respir. Dis. 1987, 136, 276-280.

28. Choe, N.; Tanaka, S.; Xia, W.; Hemenway, D.R.; Roggli, V.L.; Kagan, E. Pleural macrophage recruitment and activation in asbestos-induced pleural injury. Environ. Health Perspect. 1997, 105, 1257-1260. 
29. Liu, X.; Hurt, R.H.; Kane, A.B. Biodurability of single-walled carbon nanotubes depends on surface functionalization. Carbon 2010, 48, 1961-1969.

30. DeLorme, M.P.; Muro, Y.; Arai, T.; Banas, D.A.; Frame, S.R.; Reed, K.L.; Warheit, D.B. Ninety-day inhalation toxicity study with a vapor grown carbon nanofiber in rats. Toxicol. Sci. 2012, 128, 449-460.

31. Mitchell, L.A.; Gao, J.; Vander Wal, R.; Gigliotti, A.; Burchiel, S.W.; McDonald, J.D. Pulmonary and systemic immune response to inhaled multiwalled carbon nanotubes. Toxicol. Sci. 2007, 100, 203-214.

32. Ryman-Rasmussen, J.P.; Tewksbury, E.W.; Moss, O.R.; Cesta, M.F.; Wong, B.A.; Bonner, J.C. Inhaled multiwalled carbon nanotubes potentiate airway fibrosis in murine allergic asthma. Am. J. Respir. Cell Mol. Biol. 2009, 40, 349-358.

33. Ma-Hock, L.; Treumann, S.; Strauss, V.; Brill, S.; Luizi, F.; Mertler, M.; Wiench, K.; Gamer, A.O.; van Ravenzwaay, B.; Landsiedel, R. Inhalation toxicity of multiwall carbon nanotubes in rats exposed for 3 months. Toxicol. Sci. 2009, 112, 468-481.

34. Ryman-Rasmussen, J.P.; Cesta, M.F.; Brody, A.R.; Shipley-Phillips, J.K.; Everitt, J.I.; Tewksbury, E.W.; Moss, O.R.; Wong, B.A.; Dodd, D.E.; Andersen, M.E.; et al. Inhaled carbon nanotubes reach the subpleural tissue in mice. Nat. Nanotechnol. 2009, 4, 747-751.

35. Mercer, R.R.; Scabilloni, J.; Wang, L.; Kisin, E.; Murray, A.R.; Schwegler-Berry, D.; Shvedova, A.A.; Castranova, V. Alteration of deposition pattern and pulmonary response as a result of improved dispersion of aspirated single-walled carbon nanotubes in a mouse model. Am. J. Physiol. Lung Cell. Mol. Physiol. 2008, 294, L87-L97.

36. Mercer, R.; Hubbs, A.; Scabilloni, J.; Wang, L.; Battelli, L.; Schwegler-Berry, D.; Castranova, V.; Porter, D. Distribution and persistence of pleural penetrations by multi-walled carbon nanotubes. Part. Fibre Toxicol. 2010, 7, 28.

37. Mercer, R.; Hubbs, A.; Scabilloni, J.; Wang, L.; Battelli, L.; Friend, S.; Castranova, V.; Porter, D. Pulmonary fibrotic response to aspiration of multi-walled carbon nanotubes. Part. Fibre Toxicol. 2011, 8, 21.

38. Mercer, R.; Scabilloni, J.; Hubbs, A.; Wang, L.; Battelli, L.; McKinney, W.; Castranova, V.; Porter, D. Extrapulmonary transport of MWCNT following inhalation exposure. Part. Fibre Toxicol. 2013, 10, 38.

39. Mercer, R.; Scabilloni, J.; Hubbs, A.; Battelli, L.; McKinney, W.; Friend, S.; Wolfarth, M.; Andrew, M.; Castranova, V.; Porter, D. Distribution and fibrotic response following inhalation exposure to multi-walled carbon nanotubes. Part. Fibre Toxicol. 2013, 10, 33.

40. Porter, D.W.; Hubbs, A.F.; Chen, B.T.; McKinney, W.; Mercer, R.R.; Wolfarth, M.G.; Battelli, L.; Wu, N.; Sriram, K.; Leonard, S.; et al. Acute pulmonary dose-responses to inhaled multi-walled carbon nanotubes. Nanotoxicology 2013, 7, 1179-1194.

41. Muller, J.; Huaux, F.; Moreau, N.; Misson, P.; Heilier, J.-F.; Delos, M.; Arras, M.; Fonseca, A.; Nagy, J.B.; Lison, D. Respiratory toxicity of multi-wall carbon nanotubes. Toxicol. Appl. Pharmacol. 2005, 207, 221-231. 
42. Rothen-Rutishauser, B.; Brown, D.M.; Piallier-Boyles, M.; Kinloch, I.A.; Windle, A.H.; Gehr, P.; Stone, V. Relating the physicochemical characteristics and dispersion of multiwalled carbon nanotubes in different suspension media to their oxidative reactivity in vitro and inflammation in vivo. Nanotoxicology 2010, 4, 331-342.

43. Shvedova, A.A.; Kisin, E.R.; Mercer, R.; Murray, A.R.; Johnson, V.J.; Potapovich, A.I.; Tyurina, Y.Y.; Gorelik, O.; Arepalli, S.; Schwegler-Berry, D.; et al. Unusual inflammatory and fibrogenic pulmonary responses to single-walled carbon nanotubes in mice. Am. J. Physiol. Lung Cell. Mol. Physiol. 2005, 289, L698-L708.

44. Lam, C.W.; James, J.T.; McCluskey, R.; Hunter, R.L. Pulmonary toxicity of single-wall carbon nanotubes in mice 7 and 90 days after intratracheal instillation. Toxicol. Sci. 2004, 77, 126-134.

45. Shvedova, A.A.; Kisin, E.R.; Murray, A.R.; Gorelik, O.; Arepalli, S.; Castranova, V.; Young, S.-H.; Gao, F.; Tyurina, Y.Y.; Oury, T.D.; et al. Vitamin E deficiency enhances pulmonary inflammatory response and oxidative stress induced by single-walled carbon nanotubes in C57BL/6 mice. Toxicol. Appl. Pharmacol. 2007, 221, 339-348.

46. Wang, L.; Castranova, V.; Mishra, A.; Chen, B.; Mercer, R.; Schwegler-Berry, D.; Rojanasakul, Y. Dispersion of single-walled carbon nanotubes by a natural lung surfactant for pulmonary in vitro and in vivo toxicity studies. Part. Fibre Toxicol. 2010, 7, 31.

47. Murray, A.R.; Kisin, E.R.; Tkach, A.V.; Yanamala, N.; Mercer, R.; Young, S.H.; Fadeel, B.; Kagan, V.E.; Shvedova, A.A. Factoring-in agglomeration of carbon nanotubes and nanofibers for better prediction of their toxicity versus asbestos. Part. Fibre Toxicol. 2012, 9, 10.

48. Crouzier, D.; Follot, S.; Gentilhomme, E.; Flahaut, E.; Arnaud, R.; Dabouis, V.; Castellarin, C.; Debouzy, J.C. Carbon nanotubes induce inflammation but decrease the production of reactive oxygen species in lung. Toxicology 2010, 272, 39-45.

49. Jacobsen, N.; Moller, P.; Jensen, K.; Vogel, U.; Ladefoged, O.; Loft, S.; Wallin, H. Lung inflammation and genotoxicity following pulmonary exposure to nanoparticles in ApoE-/- mice. Part. Fibre Toxicol. 2009, 6, 2.

50. Mangum, J.; Turpin, E.; Antao-Menezes, A.; Cesta, M.; Bermudez, E.; Bonner, J. Single-Walled Carbon Nanotube (SWCNT)-induced interstitial fibrosis in the lungs of rats is associated with increased levels of PDGF mRNA and the formation of unique intercellular carbon structures that bridge alveolar macrophages in situ. Part. Fibre Toxicol. 2006, 3, 15 .

51. Shvedova, A.A.; Kisin, E.R.; Murray, A.R.; Kommineni, C.; Castranova, V.; Fadeel, B.; Kagan, V.E. Increased accumulation of neutrophils and decreased fibrosis in the lung of NADPH oxidase-deficient C57BL/ 6 mice exposed to carbon nanotubes. Toxicol. Appl. Pharmacol. 2008, 231, 235-240.

52. Cesta, M.F.; Ryman-Rasmussen, J.P.; Wallace, D.G.; Masinde, T.; Hurlburt, G.; Taylor, A.J.; Bonner, J.C. Bacterial lipopolysaccharide enhances PDGF signaling and pulmonary fibrosis in rats exposed to carbon nanotubes. Am. J. Respir. Cell Mol. Biol. 2010, 43, 142-151. 
53. Nygaard, U.C.; Samuelsen, M.; Marioara, C.D.; Lovik, M. Carbon nanofibers have IgE adjuvant capacity but are less potent than nanotubes in promoting allergic airway responses. Biomed. Res. Int. 2013, 2013, 476010.

54. Shvedova, A.A.; Kisin, E.; Murray, A.R.; Johnson, V.J.; Gorelik, O.; Arepalli, S.; Hubbs, A.F.; Mercer, R.R.; Keohavong, P.; Sussman, N.; et al. Inhalation vs. Aspiration of Single-Walled Carbon Nanotubes in C57BL/ 6 Mice: Inflammation, Fibrosis, Oxidative Stress, and Mutagenesis. Am J Physiol Lung Cell Mol Physiol. 2008, 295, L552-L565.

55. Kobayashi, N.; Naya, M.; Ema, M.; Endoh, S.; Maru, J.; Mizuno, K.; Nakanishi, J. Biological response and morphological assessment of individually dispersed multi-wall carbon nanotubes in the lung after intratracheal instillation in rats. Toxicology 2010, 276, 143-153.

56. Valko, M.; Morris, H.; Cronin, M.T.D. Metals, toxicity and oxidative stress. Curr. Med. Chem. 2005, 12, 1161-1208.

57. Hansen, K.; Mossman, B. Generation of superoxide (O2-.) from alveolar macrophages exposed to asbestiform and nonfibrous particles. Cancer Res. 1987, 47, 1681-1686.

58. Hill, I.M.; Beswick, P.H.; Donaldson, K. Differential release of superoxide anions by macrophages treated with long and short fibre amosite asbestos is a consequence of differential affinity for opsonin. Occup. Environ. Med. 1995, 52, 92-96.

59. Boyles, M.S.P.; Young, L.; Brown, D.M.; MacCalman, L.; Cowie, H.; Moisala, A.; Smail, F.; Smith, P.J.W.; Proudfoot, L.; Windle, A.H.; et al. Length Dependent Effects of Multi-Walled Carbon Nanotubes on Macrophage Mediated Inflammation, Phagocytosis and Cytotoxicity-A Comparison with Asbestos and Nanoparticle Carbon Black, submitted.

60. Muller, J.; Huaux, F.; Fonseca, A.; Nagy, J.B.; Moreau, N.; Delos, M.; Raymundo-Piñero, E.; Béguin, F.; Kirsch-Volders, M.; Fenoglio, I.; et al. Structural defects play a major role in the acute lung toxicity of multiwall carbon nanotubes: Toxicological aspects. Chem. Res. Toxicol. 2008, 21, 1698-1705.

61. Maynard, A.D.; Baron, P.A.; Foley, M.; Shvedova, A.A.; Kisin, E.R.; Castranova, V. Exposure to carbon nanotube material: Aerosol release during the handling of unrefined single-walled carbon nanotube material. J. Toxicol. Environ. Health Part A 2004, 67, 87-107.

62. Bernstein, D.; Castranova, V.; Donaldson, K.; Fubini, B.; Hadley, J.; Hesterberg, T.; Kane, A.; Lai, D.; McConnell, E.E.; Muhle, H.; et al. Testing of fibrous particles: Short-term assays and strategies. Inhal. Toxicol. 2005, 17, 497-537.

63. Brown, D.; Donaldson, K.; Stone, V. Nuclear translocation of Nrf2 and expression of antioxidant defence genes in THP-1 cells exposed to carbon nanotubes. J. Biomed. Nanotechnol. 2010, 6, 224-233.

64. Murphy, F.A.; Poland, C.A.; Duffin, R.; Al-Jamal, K.T.; Ali-Boucetta, H.; Nunes, A.; Byrne, F.; Prina-Mello, A.; Volkov, Y.; Li, S.; et al. Length-dependent retention of carbon nanotubes in the pleural space of mice initiates sustained inflammation and progressive fibrosis on the parietal pleura. Am. J. Pathol. 2011, 178, 2587-2600. 
65. Snyder-Talkington, B.N.; Dymacek, J.; Porter, D.W.; Wolfarth, M.G.; Mercer, R.R.; Pacurari, M.; Denvir, J.; Castranova, V.; Qian, Y.; Guo, N.L. System-based identification of toxicity pathways associated with multi-walled carbon nanotube-induced pathological responses. Toxicol. Appl. Pharmacol. 2013, 272, 476-489.

66. Meunier, E.; Coste, A.; Olagnier, D.; Authier, H.; Lefèvre, L.; Dardenne, C.; Bernad, J.; Béraud, M.; Flahaut, E.; Pipy, B. Double-walled carbon nanotubes trigger IL-1 $\beta$ release in human monocytes through Nlrp3 inflammasome activation. Nanomedicine 2012, 8, 987-995.

67. Besnard, A.-G.; Togbe, D.; Couillin, I.; Tan, Z.; Zheng, S.G.; Erard, F.; le Bert, M.; Quesniaux, V.; Ryffel, B. Inflammasome-IL-1-Th17 response in allergic lung inflammation. J. Mol. Cell Biol. 2012, 4, 3-10.

68. Palomäki, J.; Välimäki, E.; Sund, J.; Vippola, M.; Clausen, P.A.; Jensen, K.A.; Savolainen, K.; Matikainen, S.; Alenius, H. Long, needle-like carbon nanotubes and asbestos activate the NLRP3 inflammasome through a similar mechanism. ACS Nano 2011, 5, 6861-6870.

69. Clift, M.J.; Endes, C.; Vanhecke, D.; Wick, P.; Gehr, P.; Schins, R.P.; Petri-Fink, A.; Rothen-Rutishauser, B. A comparative study of different in vitro lung cell culture systems to assess the most beneficial tool for screening the potential adverse effects of carbon nanotubes. Toxicol. Sci. 2014, 137, 55-64.

70. Müller, L.; Riediker, M.; Wick, P.; Mohr, M.; Gehr, P.; Rothen-Rutishauser, B. Oxidative stress and inflammation response after nanoparticle exposure: Differences between human lung cell monocultures and an advanced three-dimensional model of the human epithelial airways. J. R. Soc. Interface 2010, 7, S27-S40.

71. Gasser, M.; Wick, P.; Clift, M.; Blank, F.; Diener, L.; Yan, B.; Gehr, P.; Krug, H.; Rothen-Rutishauser, B. Pulmonary surfactant coating of multi-walled carbon nanotubes (MWCNTs) influences their oxidative and pro-inflammatory potential in vitro. Part. Fibre Toxicol. 2012, 9, 1-13.

72. Chen, X.; Kis, A.; Zettl, A.; Bertozzi, C.R. A cell nanoinjector based on carbon nanotubes. Proc. Natl. Acad. Sci. USA 2007, 104, 8218-8222.

73. Fabbro, C.; Ali-Boucetta, H.; Da Ros, T.; Kostarelos, K.; Bianco, A.; Prato, M. Targeting carbon nanotubes against cancer. Chem. Commun. (Camb) 2012, 48, 3911-3926.

74. Kam, N.W.; Dai, H. Carbon nanotubes as intracellular protein transporters: Generality and biological functionality. J. Am. Chem. Soc. 2005, 127, 6021-6026.

75. Pantarotto, D.; Briand, J.P.; Prato, M.; Bianco, A. Translocation of bioactive peptides across cell membranes by carbon nanotubes. Chem. Commun. (Camb) 2004, 1, 16-17.

76. Singh, R.; Pantarotto, D.; McCarthy, D.; Chaloin, O.; Hoebeke, J.; Partidos, C.D.; Briand, J.P.; Prato, M.; Bianco, A.; Kostarelos, K. Binding and condensation of plasmid DNA onto functionalized carbon nanotubes: Toward the construction of nanotube-based gene delivery vectors. J. Am. Chem. Soc. 2005, 127, 4388-4396.

77. Meng, J.; Meng, J.; Duan, J.; Kong, H.; Li, L.; Wang, C.; Xie, S.; Chen, S.; Gu, N.; $\mathrm{Xu}, \mathrm{H}$.; et al. Carbon nanotubes conjugated to tumor lysate protein enhance the efficacy of an antitumor immunotherapy. Small 2008, 4, 1364-1370. 
78. Villa, C.H.; Dao, T.; Ahearn, I.; Fehrenbacher, N.; Casey, E.; Rey, D.A.; Korontsvit, T.; Zakhaleva, V.; Batt, C.A.; Philips, M.R.; et al. Single-walled carbon nanotubes deliver peptide antigen into dendritic cells and enhance $\operatorname{IgG}$ responses to tumor-associated antigens. ACS Nano 2011, 5, 5300-5311.

79. Zeinali, M.; Jammalan, M.; Ardestani, S.K.; Mosaveri, N. Immunological and cytotoxicological characterization of tuberculin purified protein derivative (PPD) conjugated to single-walled carbon nanotubes. Immunol. Lett. 2009, 126, 48-53.

80. Yandar, N.; Pastorin, G.; Prato, M.; Bianco, A.; Patarroyo, M.E.; Lozano, J.M. Immunological profile of a Plasmodium vivax AMA-1 N-terminus peptide-carbon nanotube conjugate in an infected Plasmodium berghei mouse model. Vaccine 2008, 26, 5864-5873.

81. Parra, J.; Abad-Somovilla, A.; Mercader, J.V.; Taton, T.A.; Abad-Fuentes, A. Carbon nanotube-protein carriers enhance size-dependent self-adjuvant antibody response to haptens. J. Control. Release 2013, 170, 242-251.

82. Klumpp, C.; Kostarelos, K.; Prato, M.; Bianco, A. Functionalized carbon nanotubes as emerging nanovectors for the delivery of therapeutics. Biochim. Biophys. Acta 2006, 1758, 404-412.

83. Abe, S.; Itoh, S.; Hayashi, D.; Kobayashi, T.; Kiba, T.; Akasaka, T.; Uo, M.; Yawaka, Y.; Sato, S.; Watari, F.; et al. Biodistribution of aqueous suspensions of carbon nanotubes in mice and their biocompatibility. J. Nanosci. Nanotechnol. 2012, 12, 700-706.

84. Dobrovolskaia, M.A.; McNeil, S.E. Handbook of Immunological Properties of Engineered Nanomaterials; World Scientific: Singapore, Singapore, 2013; p. 692 S.

85. Zolnik, B.S.; Gonzalez-Fernandez, A.; Sadrieh, N.; Dobrovolskaia, M.A. Nanoparticles and the immune system. Endocrinology 2010, 151, 458-465.

86. Radomski, A.; Jurasz, P.; Alonso-Escolano, D.; Drews, M.; Morandi, M.; Malinski, T.; Radomski, M.W. Nanoparticle-induced platelet aggregation and vascular thrombosis. Br. J. Pharmacol. 2005, 146, 882-893.

87. Lacerda, S.H.; Semberova, J.; Holada, K.; Simakova, O.; Hudson, S.D.; Simak, J. Carbon nanotubes activate store-operated calcium entry in human blood platelets. ACS Nano 2011, 5, 5808-5813.

88. Zhang, C.-H.; Luo, Y.-L.; Chen, Y.-S.; Wei, Q.-B.; Fan, L.-H. Preparation and theophylline delivery applications of novel PMAA/MWCNT-COOH nanohybrid hydrogels. J. Biomater. Sci. Polym. Ed. 2009, 20, 1119-1135.

89. Yamaguchi, A.; Fujitani, T.; Ohyama, K.; Nakae, D.; Hirose, A.; Nishimura, T.; Ogata, A. Effects of sustained stimulation with multi-wall carbon nanotubes on immune and inflammatory responses in mice. J. Toxicol. Sci. 2012, 37, 177-189.

90. Rodriguez, L.L.; Gay, C.G. Development of vaccines toward the global control and eradication of foot-and-mouth disease. Expert Rev. Vaccines 2011, 10, 377-387.

91. Bianco, A.; Hoebeke, J.; Godefroy, S.; Chaloin, O.; Pantarotto, D.; Briand, J.-P.; Muller, S.; Prato, M.; Partidos, C.D. Cationic carbon nanotubes bind to CpG oligodeoxynucleotides and enhance their immunostimulatory properties. J. Am. Chem. Soc. 2005, 127, 58-59. 
92. Zhao, D.; Alizadeh, D.; Zhang, L.; Liu, W.; Farrukh, O.; Manuel, E.; Diamond, D.J.; Badie, B. Carbon nanotubes enhance $\mathrm{CpG}$ uptake and potentiate antiglioma immunity. Clin. Cancer Res. 2011, 17, 771-782.

93. Park, E.J.; Cho, W.S.; Jeong, J.; Yi, J.; Choi, K.; Park, K. Pro-inflammatory and potential allergic responses resulting from $\mathrm{B}$ cell activation in mice treated with multi-walled carbon nanotubes by intratracheal instillation. Toxicology 2009, 259, 113-121.

94. Andersen, A.J.; Wibroe, P.P.; Moghimi, S.M. Perspectives on carbon nanotube-mediated adverse immune effects. Adv. Drug Deliv. Rev. 2012, 64, 1700-1705.

95. Bottini, M.; Bruckner, S.; Nika, K.; Bottini, N.; Bellucci, S.; Magrini, A.; Bergamaschi, A.; Mustelin, T. Multi-walled carbon nanotubes induce T lymphocyte apoptosis. Toxicol. Lett. 2006, 160, 121-126.

96. Inoue, K.; Koike, E.; Yanagisawa, R.; Hirano, S.; Nishikawa, M.; Takano, H. Effects of multi-walled carbon nanotubes on a murine allergic airway inflammation model. Toxicol. Appl. Pharmacol. 2009, 237, 306-316.

97. Nygaard, U.C.; Hansen, J.S.; Samuelsen, M.; Alberg, T.; Marioara, C.D.; Løvik, M. Single-walled and multi-walled carbon nanotubes promote allergic immune responses in mice. Toxicol. Sci. 2009, 109, 113-123.

98. Meng, J.; Yang, M.; Jia, F.; Xu, Z.; Kong, H.; Xu, H. Immune responses of BALB/c mice to subcutaneously injected multi-walled carbon nanotubes. Nanotoxicology 2011, 5, 583-591.

99. Meng, J.; Yang, M.; Jia, F.; Kong, H.; Zhang, W.; Wang, C.; Xing, J.; Xie, S.; Xu, H. Subcutaneous injection of water-soluble multi-walled carbon nanotubes in tumor-bearing mice boosts the host immune activity. Nanotechnology 2010, 21, 145104.

100. Yokoyama, A.; Sato, Y.; Nodasaka, Y.; Yamamoto, S.; Kawasaki, T.; Shindoh, M.; Kohgo, T.; Akasaka, T.; Uo, M.; Watari, F.; et al. Biological behavior of hat-stacked carbon nanofibers in the subcutaneous tissue in rats. Nano Lett. 2005, 5, 157-161.

101. Sato, Y.; Yokoyama, A.; Nodasaka, Y.; Kohgo, T.; Motomiya, K.; Matsumoto, H.; Nakazawa, E.; Numata, T.; Zhang, M.; Yudasaka, M.; et al. Long-term biopersistence of tangled oxidized carbon nanotubes inside and outside macrophages in rat subcutaneous tissue. Sci. Rep. 2013, 3, 2516.

102. Cheng, W.W.; Lin, Z.Q.; Wei, B.F.; Zeng, Q.; Han, B.; Wei, C.X.; Fan, X.J.; Hu, C.L.; Liu, L.H.; Huang, J.H.; et al. Single-walled carbon nanotube induction of rat aortic endothelial cell apoptosis: Reactive oxygen species are involved in the mitochondrial pathway. Int. J. Biochem. Cell Biol. 2011, 43, 564-572.

103. Guo, X.; Jagannath, C.; Espitia, M.G.; Zhou, X. Uptake of silica and carbon nanotubes by human macrophages/monocytes induces activation of fibroblasts in vitro-Potential implication for pathogenesis of inflammation and fibrotic diseases. Int. J. Immunopathol. Pharmacol. 2012, 25, 713-719.

104. Albini, A.; Mussi, V.; Parodi, A.; Ventura, A.; Principi, E.; Tegami, S.; Rocchia, M.; Francheschi, E.; Sogno, I.; Cammarota, R.; et al. Interactions of single-wall carbon nanotubes with endothelial cells. Nanomedicine 2010, 6, 277-288. 
105. Pulskamp, K.; Diabate, S.; Krug, H.F. Carbon nanotubes show no sign of acute toxicity but induce intracellular reactive oxygen species in dependence on contaminants. Toxicol. Lett. 2007, 168, 58-74.

106. Vlasova, I.I.; Vakhrusheva, T.V.; Sokolov, A.V.; Kostevich, V.A.; Gusev, A.A.; Gusev, S.A.; Melnikova, V.I.; Lobach, A.S. PEGylated single-walled carbon nanotubes activate neutrophils to increase production of hypochlorous acid, the oxidant capable of degrading nanotubes. Toxicol. Appl. Pharmacol. 2012, 264, 131-142.

107. Sun, Z.; Liu, Z.; Meng, J.; Meng, J.; Duan, J.; Xie, S.; Lu, X.; Zhu, Z.; Wang, C.; Chen, S.; et al . Carbon nanotubes enhance cytotoxicity mediated by human lymphocytes in vitro. PLoS One 2011, 6, e21073.

108. Clichici, S.; Biris, A.R.; Tabaran, F.; Filip, A. Transient oxidative stress and inflammation after intraperitoneal administration of multiwalled carbon nanotubes functionalized with single strand DNA in rats. Toxicol. Appl. Pharmacol. 2012, 259, 281-292.

109. Yuan, J.; Gao, H.; Sui, J.; Duan, H.; Chen, W.N.; Ching, C.B. Cytotoxicity evaluation of oxidized single-walled carbon nanotubes and graphene oxide on human hepatoma HepG2 cells: An iTRAQ-coupled 2D LC-MS/MS proteome analysis. Toxicol. Sci. 2012, 126, 149-161.

110. Kermanizadeh, A.; Gaiser, B.K.; Hutchison, G.R.; Stone, V. An in vitro liver model-Assessing oxidative stress and genotoxicity following exposure of hepatocytes to a panel of engineered nanomaterials. Part. Fibre Toxicol. 2012, 9, 28.

111. Kermanizadeh, A.; Pojana, G.; Gaiser, B.K.; Birkedal, R.; Bilanicová, D.; Wallin, H.; Jensen, K.A.; Sellergren, B.; Hutchison, G.R.; Marcomini, A.; et al. In vitro assessment of engineered nanomaterials using a hepatocyte cell line: Cytotoxicity, pro-inflammatory cytokines and functional markers. Nanotoxicology 2013, 7, 301-313.

112. Demento, S.L.; Eisenbarth, S.C.; Foellmer, H.G.; Platt, C.; Caplan, M.J.; Mark Saltzman, W.; Mellman, I.; Ledizet, M.; Fikrig, E.; Flavell, R.A.; et al. Inflammasome-activating nanoparticles as modular systems for optimizing vaccine efficacy. Vaccine 2009, 27, 3013-3021.

113. Boraschi, D.; Duschl, A. Nanoparticles and the Immune System-Safety and Effects; Adademic Press: Oxford, UK, 2013.

114. Gao, N.; Zhang, Q.; Mu, Q.; Bai, Y.; Li, L.; Zhou, H.; Butch, E.R.; Powell, T.B.; Snyder, S.E.; Jiang, G.; et al. Steering carbon nanotubes to scavenger receptor recognition by nanotube surface chemistry modification partially alleviates NFkappaB activation and reduces its immunotoxicity. ACS Nano 2011, 5, 4581-4591.

115. Moghimi, S.M.; Andersen, A.J.; Ahmadvand, D.; Wibroe, P.P.; Andresen, T.L.; Hunter, A.C. Material properties in complement activation. Adv. Drug Deliv. Rev. 2011, 63, 1000-1007.

116. Salvador-Morales, C.; Flahaut, E.; Sim, E.; Sloan, J.; Green, M.L.; Sim, R.B. Complement activation and protein adsorption by carbon nanotubes. Mol. Immunol. 2006, 43, 193-201.

117. Rybak-Smith, M.J.; Sim, R.B. Complement activation by carbon nanotubes. Adv. Drug Deliv. Rev. 2011, 63, 1031-1041. 
118. Carroll, M.V.; Sim, R.B. Complement in health and disease. Adv. Drug Deliv. Rev. 2011, 63, 965-975.

119. Ling, W.L.; Biro, A.; Bally, I.; Tacnet, P.; Deniaud, A.; Doris, E.; Frachet, P.; Schoehn, G.; Pebay-Peyroula, E.; Arlaud, G.J. Proteins of the innate immune system crystallize on carbon nanotubes but are not activated. ACS Nano 2011, 5, 730-737.

120. Andersen, A.J.; Robinson, J.T.; Dai, H.; Hunter, A.C.; Andresen, T.L.; Moghimi, S.M. Single-walled carbon nanotube surface control of complement recognition and activation. ACS Nano 2013, 7, 1108-1119.

121. Rybak-Smith, M.J.; Tripisciano, C.; Borowiak-Palen, E.; Lamprecht, C.; Sim, R.B. Effect of functionalization of carbon nanotubes with psychosine on complement activation and protein adsorption. J. Biomed. Nanotechnol. 2011, 7, 830-839.

122. Čolić, M.; Džopalić, T.; Tomić, S.; Rajković, J.; Rudolf, R.; Vuković, G.; Marinković, A.; Uskoković, P. Immunomodulatory effects of carbon nanotubes functionalized with a Toll-like receptor 7 agonist on human dendritic cells. Carbon 2014, 67, 273-287.

123. Gottardi, R.; Douradinha, B. Carbon nanotubes as a novel tool for vaccination against infectious diseases and cancer. J. Nanobiotechnology 2013, 11, 30.

124. Casals, E.; Pfaller, T.; Duschl, A.; Oostingh, G.J.; Puntes, V. Time evolution of the nanoparticle protein corona. ACS Nano 2010, 4, 3623-3632.

125. Monopoli, M.P.; Aberg, C.; Salvati, A.; Dawson, K.A. Biomolecular coronas provide the biological identity of nanosized materials. Nat. Nanotechnol. 2012, 7, 779-786.

126. Shannahan, J.H.; Brown, J.M.; Chen, R.; Ke, P.C.; Lai, X.; Mitra, S.; Witzmann, F.A. Comparison of nanotube-protein corona composition in cell culture media. Small 2013, 9 , 2171-2181.

127. Tenzer, S.; Docter, D.; Kuharev, J.; Musyanovych, A.; Fetz, V.; Hecht, R.; Schlenk, F.; Fischer, D.; Kiouptsi, K.; Reinhardt, C.; et al. Rapid formation of plasma protein corona critically affects nanoparticle pathophysiology. Nat. Nanotechnol. 2013, 8, 772-781.

128. Laverny, G.; Casset, A.; Purohit, A.; Schaeffer, E.; Spiegelhalter, C.; de Blay, F.; Pons, F. Immunomodulatory properties of multi-walled carbon nanotubes in peripheral blood mononuclear cells from healthy subjects and allergic patients. Toxicol. Lett. 2013, 217, 91-101.

129. Palomaki, J.; Karisola, P.; Pylkkanen, L.; Savolainen, K.; Alenius, H. Engineered nanomaterials cause cytotoxicity and activation on mouse antigen presenting cells. Toxicology 2010, 267, 125-131.

130. Aldinucci, A.; Turco, A.; Biagioli, T.; Toma, F.M.; Bani, D.; Guasti, D.; Manuelli, C.; Rizzetto, L.; Cavalieri, D.; Massacesi, L.; et al. Carbon nanotube scaffolds instruct human dendritic cells: Modulating immune responses by contacts at the nanoscale. Nano Lett. 2013, 13, 6098-6105.

131. Thurnherr, T.; Brandenberger, C.; Fischer, K.; Diener, L.; Manser, P.; Maeder-Althaus, X.; Kaiser, J.P.; Krug, H.F.; Rothen-Rutishauser, B.; Wick, P. A comparison of acute and long-term effects of industrial multiwalled carbon nanotubes on human lung and immune cells in vitro. Toxicol. Lett. 2011, 200, 176-186. 
132. Alam, A.; Sachar, S.; Puri, N.; Saxena, R.K. Interactions of polydispersed single-walled carbon nanotubes with $\mathrm{T}$ cells resulting in downregulation of allogeneic CTL responses in vitro and in vivo. Nanotoxicology 2013, 7, 1351-1360.

133. Erlanger, B.F.; Chen, B.; Zhu, M.; Brus, L. Binding of an anti-fullerene IgG monoclonal antibody to single wall carbon nanotubes. Nano Lett. 2001, 1, 465-467.

134. Pantarotto, D.; Partidos, C.D.; Hoebeke, J.; Brown, F.; Kramer, E.; Briand, J.P.; Muller, S.; Prato, M.; Bianco, A. Immunization with peptide-functionalized carbon nanotubes enhances virus-specific neutralizing antibody responses. Chem. Biol. 2003, 10, 961-966.

135. Delogu, L.G.; Venturelli, E.; Manetti, R.; Pinna, G.A.; Carru, C.; Madeddu, R.; Murgia, L.; Sgarrella, F.; Dumortier, H.; Bianco, A. Ex vivo impact of functionalized carbon nanotubes on human immune cells. Nanomedicine (Lond) 2012, 7, 231-243.

136. Dumortier, H.; Lacotte, S.; Pastorin, G.; Marega, R.; Wu, W.; Bonifazi, D.; Briand, J.P.; Prato, M.; Muller, S.; Bianco, A. Functionalized carbon nanotubes are non-cytotoxic and preserve the functionality of primary immune cells. Nano Lett. 2006, 6, 1522-1528.

137. Medepalli, K.; Alphenaar, B.; Raj, A.; Sethu, P. Evaluation of the direct and indirect response of blood leukocytes to carbon nanotubes (CNTs). Nanomedicine 2011, 7, 983-991.

138. Pescatori, M.; Bedognetti, D.; Venturelli, E.; Menard-Moyon, C.; Bernardini, C.; Muresu, E.; Piana, A.; Maida, G.; Manetti, R.; Sgarrella, F.; et al. Functionalized carbon nanotubes as immunomodulator systems. Biomaterials 2013, 34, 4395-4403.

139. Grecco, A.C.; Paula, R.F.; Mizutani, E.; Sartorelli, J.C.; Milani, A.M.; Longhini, A.L.; Oliveira, E.C.; Pradella, F.; Silva, V.D.; Moraes, A.S.; et al. Up-regulation of T lymphocyte and antibody production by inflammatory cytokines released by macrophage exposure to multi-walled carbon nanotubes. Nanotechnology 2011, 22, 265103.

140. Bergin, I.L.; Witzmann, F.A. Nanoparticle toxicity by the gastrointestinal route: Evidence and knowledge gaps. Int. J. Biomed. Nanosci. Nanotechnol. 2013, 3.

141. Upadhyayula, V.K.; Deng, S.G.; Mitchell, M.C.; Smith, G.B. Application of carbon nanotube technology for removal of contaminants in drinking water: A review. Sci. Total Environ. 2009, 408, 1-13.

142. Brody, A.L.; Bugusu, B.; Han, J.H.; Sand, C.K.; Mchugh, T.H. Innovative food packaging solutions. J. Food Sci. 2008, 73, R107-R116.

143. Prajapati, V.K.; Awasthi, K.; Yadav, T.P.; Rai, M.; Srivastava, O.N.; Sundar, S. An oral formulation of amphotericin $\mathrm{b}$ attached to functionalized carbon nanotubes is an effective treatment for experimental visceral leishmaniasis. J. Infect. Dis. 2012, 205, 333-336.

144. Kou, W.; Akasaka, T.; Watari, F.; Sjogren, G. An in vitro evaluation of the biological effects of carbon nanotube-coated dental zirconia. ISRN Dent. 2013, 2013, 296727.

145. Wang, Z.Y.; Zhao, J.; Song, L.; Mashayekhi, H.; Chefetz, B.; Xing, B.S. Adsorption and desorption of phenanthrene on carbon nanotubes in simulated gastrointestinal fluids. Environ. Sci. Technol. 2011, 45, 6018-6024.

146. Szendi, K.; Varga, C. Lack of genotoxicity of carbon nanotubes in a pilot study. Anticancer Res. 2008, 28, 349-352. 
147. Folkmann, J.K.; Risom, L.; Jacobsen, N.R.; Wallin, H.; Loft, S.; Moller, P. Oxidatively damaged DNA in rats exposed by oral gavage to $\mathrm{C} 60$ fullerenes and single-walled carbon nanotubes. Environ. Health Perspect. 2009, 117, 703-708.

148. Lim, J.-H.; Kim, S.-H.; Shin, I.-S.; Park, N.-H.; Moon, C.; Kang, S.-S.; Kim, S.-H.; Park, S.-C.; Kim, J.-C. Maternal exposure to multi-wall carbon nanotubes does not induce embryo-fetal developmental toxicity in rats. Birth Defects Res. Part B Dev. Reprod. Toxicol. 2011, 92, 69-76.

149. Philbrook, N.A.; Walker, V.K.; Afrooz, A.R.; Saleh, N.B.; Winn, L.M. Investigating the effects of functionalized carbon nanotubes on reproduction and development in Drosophila melanogaster and CD-1 mice. Reprod. Toxicol. 2011, 32, 442-448.

150. Matsumoto, M.; Serizawa, H.; Sunaga, M.; Kato, H.; Takahashi, M.; Hirata-Koizumi, M.; Ono, A.; Kamata, E.; Hirose, A. No toxicological effects on acute and repeated oral gavage doses of single-wall or multi-wall carbon nanotube in rats. J. Toxicol. Sci. 2012, 37, 463-474. 


\title{
Multi-Functional Magnetic Photoluminescent Photocatalytic Polystyrene-Based Micro- and Nano-Fibers Obtained by Electrospinning
}

\author{
Michel Schaer, Mireille Crittin, Lamia Kasmi, Katarzyna Pierzchala, \\ Caroline Calderone, Reinaldo G. Digigow, Alke Fink, László Forró and \\ Andrzej Sienkiewicz
}

\begin{abstract}
This work reports on the implementation of electrospinning (ES) as a facile route to encapsulate nano-engineered materials in a polystyrene (PS) matrix. We applied ES to co-encapsulate two kinds of nanoparticles, i.e., upconversion nanophosphors (UCNPs) and superparamagnetic iron oxide nanoparticles (SPIONs), in polystyrene (PS)-based micro- and nano-fibers (PSFs). This approach made it possible to integrate near-infrared (NIR) light-sensitive 500-nm $\beta-\mathrm{NaYF}_{4}: \mathrm{Yb}, \mathrm{Er}$ UCNPs with $10-\mathrm{nm} \gamma-\mathrm{Fe}_{2} \mathrm{O}_{3}$ SPIONs in PS fibers. During the ES process, PSFs were additionally loaded with a well-established singlet oxygen $\left({ }^{1} \Delta_{\mathrm{g}}\right)$ photosensitizer, rose bengal (RB). The thus obtained PSFs revealed the promising features of prospective multi-functional magnetic photoluminescent photocatalytic nano-constructs.
\end{abstract}

Reprinted from Fibers. Cite as: Schaer, M.; Crittin, M.; Kasmi, L.; Pierzchala, K.; Calderone, C.; Digigow, R.G.; Fink, A.; Forró, L.; Sienkiewicz, A. Multi-Functional Magnetic Photoluminescent Photocatalytic Polystyrene-Based Micro- and Nano-Fibers Obtained by Electrospinning. Fibers 2014, 2, 75-91.

\section{Introduction}

There is a growing interest in designing composite nanostructured materials made of inorganic nanoparticles entrapped in organic polymer matrices [1]. In particular, encapsulation of inorganic nano-engineered particles (NPs) in a polymer matrix is of a high importance in various fields of applications, such as cosmetics, paints, additives, bio-medicine, etc. [2]. In this regard, numerous polymer-based encapsulation techniques have been reported using different approaches to encapsulate various inorganic NPs, like silica, titania, alumina, calcium carbonate, carbon black, magnetic iron oxides, metal nanoparticles and quantum dots [3-6]. The controlled fabrication of well-defined micro- and nano-sized composites encapsulated in polymer matrices has become one of the main topics in materials science oriented towards bio-medical applications. In particular, polymer-based fibrillar nano-structured composites provide a connection between the nanoscale world and the macroscale world, because their diameters and encapsulated active 
components are in the nanometer range, whereas their overall lengths are in the kilometer range [7]. Fabrics designed around polymer-based nano-fibrillar composites offer a plethora of interesting properties, such as exceptionally high surface area, ease of functionalization via simple chemistry and multi-functionality, just to name a few of them. These features are of particular importance for the design and fabrication of multi-functional biomaterials, which are necessary for advanced study in bio-sensing, tissue engineering and regenerative medicine [8]. Well-defined multi-functional nanostructured fibers are also finding applications in controlled drug delivery and drug release, nano-micro-electromechanical systems, advanced filtration, etc. [9].

Electrospinning (ES) is gaining increasing attention as a versatile method of preparing polymer-based micro- and nano-fibers, as well as a facile route towards the encapsulation of nanoscale materials [10]. ES is a polymer processing technique that uses electrostatic forces to uniaxially stretch a viscoelastic jet derived from a polymer solution (or polymer melt) and delivered through a millimeter-scale nozzle to produce continuous nanometric and micrometric fibers, which are typically assembled into non-woven mats [11,12].

With respect to other nanofiber fabrication technologies, like template synthesis, self-assembly or phase separation, ES offers several advantages, including the simplicity and low-cost of the manufacturing routes, the possibility of using a great variety of polymers, both synthetic and natural, which can be processed into fibers, as well as a fairly good control of fiber mechanical properties and dimensions. ES makes it also possible to precisely monitor the fiber porosity and fiber orientation in the resulting mat. Most importantly, from the standpoint of bio-medical applications, functionalization of electrospun (E-spun) fibers can easily be integrated during their production $[13,14]$.

Over the last two decades, ES has proven to be a versatile technological route towards obtaining multifunctional polymeric nanofibers suitable for numerous biomedical applications, including drug delivery and tissue engineering [15-17]. Reports are available on E-spun multifunctional polymer nanofibers containing visible light responsive dyes, like porphyrin or phthalocyanine, which can produce electronically excited molecular oxygen (singlet oxygen, ${ }^{1} \Delta_{\mathrm{g}}$ ) upon illumination with visible light and reveal an antibacterial effect [18-20]. Such multifunctional nanofibrillar composites have been found to be suitable for the fabrication of filters for water disinfection and the preparation of wound dressing patches to combat microbial infections.

In recent years, applications of upconversion nanophosphors (UCNPs) in bio-imaging and bio-sensing have been rapidly growing. Upconversion refers to a nonlinear optical processes characterized by the successive absorption of two or more low-energy photons via intermediate long-lived energy states, which is 
then followed by emission at a shorter wavelength than that of excitation. Thus, UCNPs, generally in the form of nano-/micro-crystals of lanthanide-doped rare earth fluorides, which utilize near-infrared (NIR) light rather than ultraviolet and visible light excitation, offer numerous advantageous features in bio-imaging, including increased penetration depth of biological tissues, large anti-Stokes shifts of the emitted light, sharp emission bandwidths, minimized autofluorescence and low photodamage [21,22]. Compared to traditional organic fluorophores or semiconductor quantum dots (QDs) that are currently used in bio-imaging, UCNPs exhibit much higher photostability and the absence of blinking and photobleaching $[23,24]$. Moreover, unlike QDs-based multi-photon bio-labels, which require simultaneous absorption of several NIR photons from a high-power pulsed laser source, UCNPs generate efficient upconversion luminescence (UCL) by a continuous-wave NIR diode laser, due to their real ladder-like energy levels of trivalent lanthanide ions [25]. Until quite recently, the low UCL efficiency of small-sized nanophosphors $(<50 \mathrm{~nm})$, as compared to their bulk crystal counterparts, has been a key problem in bio-analytical applications of UCNPs [22,26]. However, the most recent rapid advances in preparation methods of lanthanide-doped rare earth fluorides have led to the development of small-sized UCNPs, which exhibit both high UCL efficiency and photostability. Such UCNPs are now becoming available for biological labeling and imagining [27-30].

Among the broad spectrum of nano-engineered materials, magnetic nanoparticles (e.g., iron, iron oxides, cobalt and nickel oxides) are gaining increasing attention for many technological and biomedical applications, including magnetic storage media, bio-sensing, targeted drug delivery, contrast agents in magnetic resonance imaging (MRI) or hyperthermia [31,32]. In these applications, superparamagnetic iron oxide nanoparticles (SPIONs), like magnetite $\left(\mathrm{Fe}_{3} \mathrm{O}_{4}\right)$ or maghemite $\left(\gamma-\mathrm{Fe}_{2} \mathrm{O}_{3}\right)$, are widely encountered, because of their low toxicity and biocompatibility. In the context of designing magnetic nanocomposites, it has recently been demonstrated that the ES technology provides a flexible and cost-effective way towards obtaining polymer-based multi-functional magnetic nanostructured fibers $[33,34]$. It has also been shown that E-spun nanocomposite magnetic fibers, which incorporate superparamagnetic SPIONs into fibrous polymeric scaffolds, open new avenues for novel biomedical applications, such as, e.g., magnetic field-stimulated bone tissue regeneration or hyperthermia treatment $[35,36]$.

In this work, we implemented the ES technology as a facile route to encapsulate two kinds of nano-engineered materials, i.e., UCNPs and SPIONs, in a polystyrene (PS) matrix. To this end, we implemented as UCNPs the commercially available particles of the hexagonal crystal phase of sodium yttrium fluoride $\left(\mathrm{NaYF}_{4}\right)$ doped with $\mathrm{Yb}^{3+}$ and $\mathrm{Er}^{3+}\left(\beta-\mathrm{NaYF}_{4}: \mathrm{Yb}, \mathrm{Er}\right)$. It is commonly accepted that the $\beta-\mathrm{NaYF}_{4}$ matrix exhibits the highest UCL efficiency among the lanthanide-doped fluorides [37]. 
In particular, under excitation with NIR light, $\beta-\mathrm{NaYF}_{4}: \mathrm{Yb}, \mathrm{Er}$ UCNPs reveal strong UCL emission bands in the green and red portions of the visible spectrum [38]. The UCNPs were co-encapsulated with the custom-synthesized $\gamma-\mathrm{Fe}_{2} \mathrm{O}_{3}$ SPIONs in the polystyrene-based matrix in the same one-step ES process. This approach made it possible to obtain NIR light and magnetic field responsive polystyrene-based microand nano-fibers (PS-based fibers).

Moreover, in the same single-step ES process, PS-based fibers were additionally loaded with a singlet oxygen $\left({ }^{1} \Delta_{\mathrm{g}}\right)$ photosensitizer, rose bengal (RB). RB, a xanthene derivative among the most efficient producers of singlet oxygen, absorbs visible light in the range from 450 to $600 \mathrm{~nm}$, with a maximum around $550 \mathrm{~nm}$ [39]. Under excitation with visible light, RB forms long-lived excited triplet states, which can effectively be quenched by molecular oxygen to produce ${ }^{1} \Delta_{\mathrm{g}}$ with a high quantum yield, $\Phi_{\Delta}$, of 0.75 in water [40]. The perfect overlap of the green UCL emission and $\mathrm{RB}$ absorption in the green spectral range clearly points to the rationale of using $\mathrm{RB}$ as a ${ }^{1} \Delta_{\mathrm{g}}$ photosensitizer in conjunction with $\beta-\mathrm{NaYF}_{4}: \mathrm{Yb}, \mathrm{Er}$ UCNPs [41].

The nano-structured morphology of the thus produced PS-based fibers was characterized by scanning electron microscopy (SEM) and atomic force microscopy (AFM). The upconversion luminescence (UCL) properties of PS-based fibers under NIR light excitation were verified using spectrofluorimetry. The magnetic properties of PS-based fibers were checked with the electron spin resonance (ESR) technique. Finally, the photodynamic efficiency of PS-based fibers towards the generation of ${ }^{1} \Delta_{\mathrm{g}}$ in aqueous media under NIR light excitation was confirmed by reactive scavenging with 2,2,6,6-tetramethyl-4-piperidinol (TMP-OH), a widely-used ${ }^{1} \Delta_{\mathrm{g}}$ scavenger, which was followed by ESR detection of the resulting paramagnetic product, 4-hydroxy-2,2,6,6-tetramethylpiperidine-1-oxyl (TEMPOL). Overall, the obtained polystyrene-based fibers (PSFs) revealed promising features, such as prospective multi-functional magnetic photoluminescent photocatalytic nano-constructs.

\section{Experimental Section}

\subsection{UCNPS}

The commercial upconversion particles, product code PTIR 550/F (PTIR 550/F), were purchased from Phosphor Technology, Ltd., Stevenage, England. These materials consist of a powdered phosphor, $\beta-\mathrm{NaYF}_{4}: \mathrm{Er}, \mathrm{Yb}$, with average grain sizes in the range of 2.6-6.1 $\mu \mathrm{m}$ [42] and a high absolute upconversion quantum yield of ca. 3\%, as compared to particles of smaller sizes [26]. Under excitation with NIR light $\left(\lambda_{\mathrm{ex}}=975 \mathrm{~nm}\right)$, PTIR 550/F emits strong and narrow UCL emission bands at 520-550 $\mathrm{nm}$ and 650-670 $\mathrm{nm}$.

The as-received PTIR 550/F material was too large in average diameter to be employed in the process of ES. Therefore, prior to any further processing, PTIR550/F 
was ground in a ball mill. The dynamic light scattering (DLS) measurements performed on the ground material pointed to the achieved average particle sizes in the range of 300-550 $\mathrm{nm}$. The TEM images of the as-received and ground PTIR 550/F, as well as the corresponding particle size distributions are shown in Figure 1.

\subsection{SPIONs}

The superparamagnetic iron oxide nanoparticles, i.e., $10-\mathrm{nm} \gamma-\mathrm{Fe}_{2} \mathrm{O}_{3}$ SPIONs, were custom-synthesized using a modified method reported by Bee et al. [43] and van Ewijk et al. [44]. SPIONs were prepared by alkaline co-precipitation of ferric and ferrous chlorides in aqueous solution, as described in detail by Chastellain et al. [45]; a representative transmission electron microscopy (TEM) image is shown in Figure 2. This synthesis route yielded a concentrated suspension of SPIONs (10 mg Fe/mL) stabilized at acidic $\mathrm{pH}\left(\mathrm{HNO}_{3}, 10^{-2} \mathrm{M}\right)$.
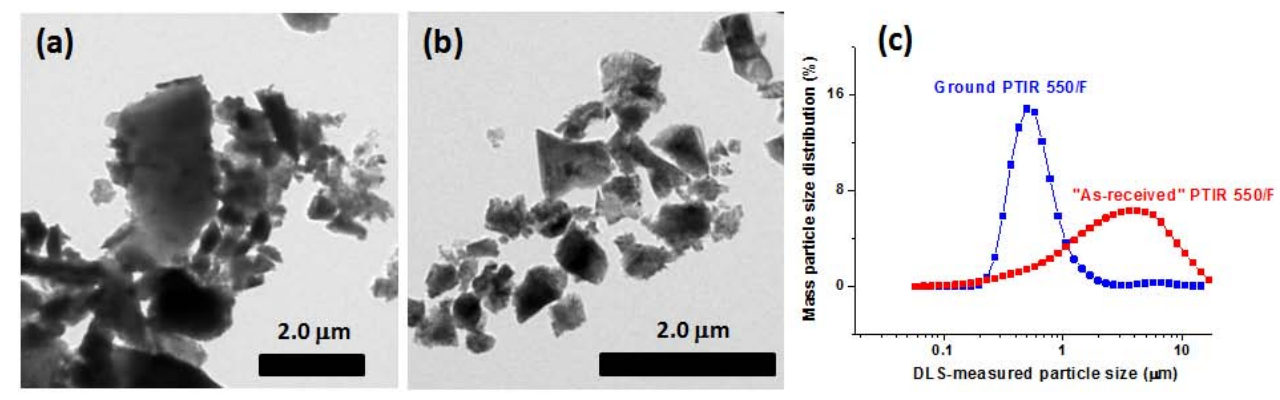

Figure 1. The TEM images obtained for the commercial upconversion particles, PTIR 550/F, before (a) and after grinding (b) and the corresponding DLS-measured particle size distributions (c).

\subsection{Preparation of PS Solutions}

Pellets of white-colored PS (molecular weight $=192,000 \mathrm{~kg} / \mathrm{kmol}$, Sigma Aldrich) were dissolved in a 1:1 mixture of acetone and cyclohexane to a concentration of $20 \mathrm{wt} \%$. Subsequently, the nanomaterials, i.e., UCNPs and SPIONs, were added to the PS solutions. The following proportions were used: UCNPs, $0.7 \mathrm{mg} / \mathrm{mL}$ of dissolved PS ( $5 \mathrm{wt} \%$ ); SPIONs, $0.1 \mathrm{~mL}$ of the custom-made ferro-fluid per $1 \mathrm{~mL}$ of dissolved PS. Finally, RB (dye content of 95\%, Sigma Aldrich, Switzerland) dissolved in ethanol/acetone was added to the PS solution to get a concentration of $c a$. $0.1 \mathrm{mg} \mathrm{RB} / \mathrm{mL}$ of the final PS solution (i.e., $\sim 100 \mu \mathrm{M}$ ). To ensure a good homogeneity of the prepared melts, the PS solutions were shaken and magnetically stirred for a prolonged time (usually more than $12 \mathrm{~h}$ ). 


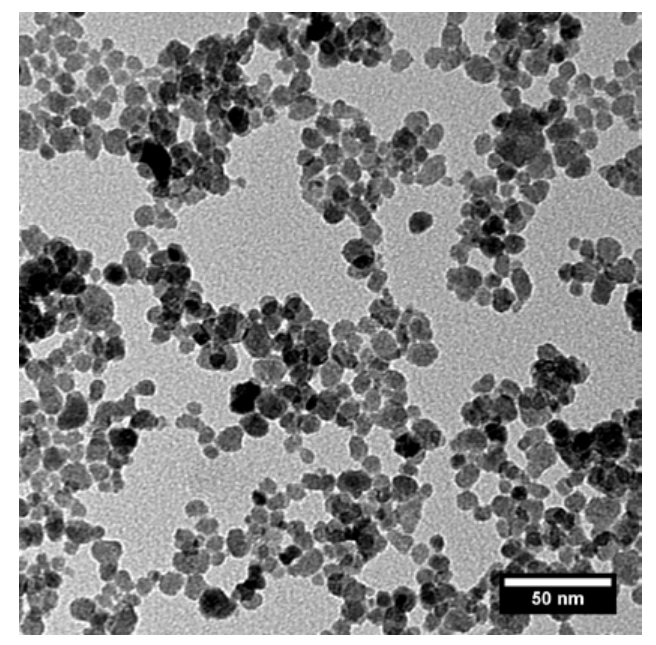

Figure 2. The TEM image of the custom-synthesized $10-\mathrm{nm} \gamma-\mathrm{Fe}_{2} \mathrm{O}_{3}$ superparamagnetic iron oxide nanoparticles (SPIONs).

\subsection{ES Apparatus}

The polystyrene-based fibers (PSFs) were produced by the ES process using a custom-built system with a high-voltage source connected to the spinneret (stainless steel nozzle) and equipped with a rotating cylindrical-in-shape ground electrode used as a target for harvesting the fibrous deposit, as schematically shown in Figure 3.

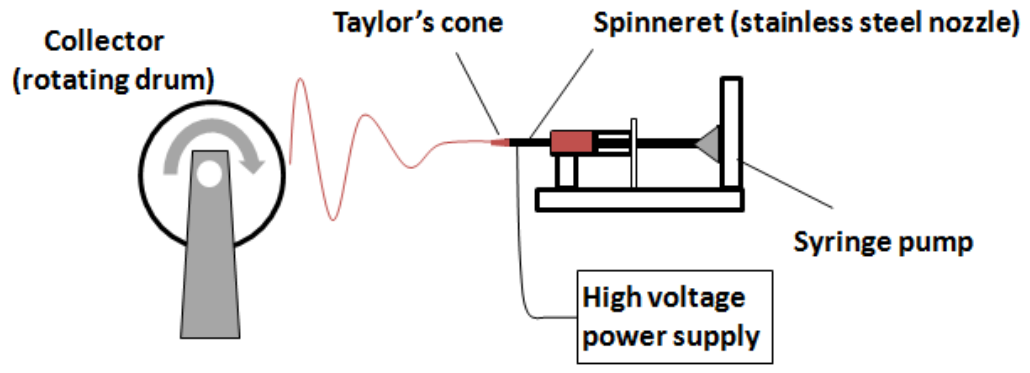

Figure 3. Schematic representation of the home-built electrospinning (ES) apparatus.

The major instrumental parameters were as follows: the applied "spinneret-collector" voltage up to $15 \mathrm{kV}$ was typically set at $8 \mathrm{kV}$; the "spinneret-collector" distance variable in the range 1 to $15 \mathrm{~cm}$, was typically set at $8 \mathrm{~cm}$; the axial translation of the collector was within the 10-cm range; the solution flow rate was manually adjustable down to $0.1 \mathrm{~mL} / \mathrm{h}$; the rotating collector speed was adjustable in the range of 60 to $1200 \mathrm{rpm}$; the spinneret diameter was of $0.3 \mathrm{~mm}$. 


\section{Results and Discussion}

The E-spun fibers formed a dense mat, which could easily be detached from the collector (rotating drum) of our ES apparatus and laid out on a flat surface. The overall morphology of a typical non-woven fabrics obtained via ES processes is shown in Figure 4a.

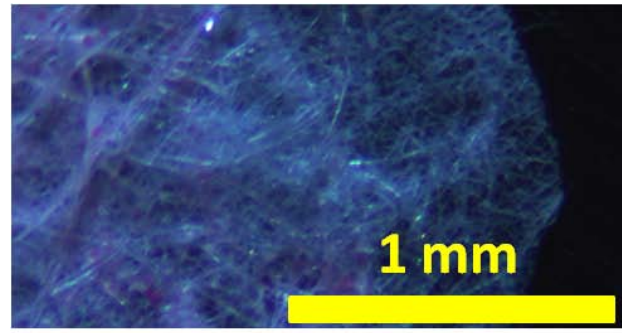

(a)

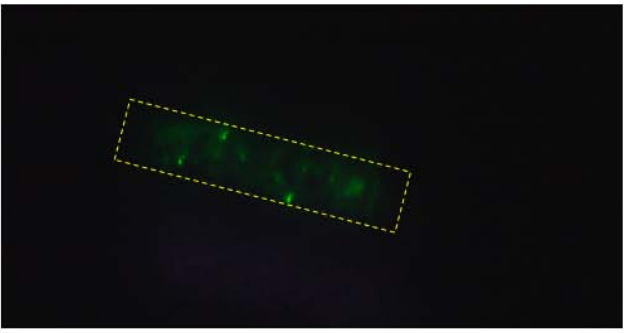

(b)

Figure 4. The morphology of a mat consisting of E-spun polystyrene (PS)-based fibers shown under visible light illumination (a) and near-infrared (NIR) light excitation (b). The rectangular contour marked by the broken green line corresponds to the rectangular shape of the incident NIR laser beam (right panel).

As can be seen in Figure $4 b$, under NIR light excitation $\left(\lambda_{\mathrm{ex}}=975 \mathrm{~nm}\right)$, the non-woven fabric emits a bright yellowish green luminescence, which can be attributed to the sum of two primary emissions bandwidths of the UCNPs, at $525 \mathrm{~nm}$ and $545 \mathrm{~nm}$ (green) and $665 \mathrm{~nm}$ (red), corresponding respectively to the radiative transitions from the ${ }^{2} \mathrm{H}_{11 / 2},{ }^{4} \mathrm{~S}_{3 / 2}$ and ${ }^{4} \mathrm{~F}_{9 / 2}$ excited states to the ${ }^{4} \mathrm{I}_{15 / 2}$ ground state of the $\mathrm{Er}^{3+}$ atom in $\beta-\mathrm{NaYF}_{4}$ : $\mathrm{Er}, \mathrm{Yb}$ [37].

The luminescence of the E-spun fibers was checked by positioning a small bundle of fibers in a light integrating sphere (general purpose 6" in diameter integrating sphere, Edmund Optics Ltd., York, UK), which was coupled via an optical fiber to a spectrofluorometer, model USB2000+XR1 (Ocean Optics, Dunedin, FL, USA). The NIR light excitation $\left(\lambda_{\mathrm{ex}}=975 \mathrm{~nm}\right)$ was performed using a 2-W variable power laser diode, model MDL-III-975 (GMP SA, Renens, Switzerland). The evolution of the luminescence spectra as a function of the NIR light excitation power for the PS-based fibers loaded with UCNPs and RB are shown in Figure 5. 


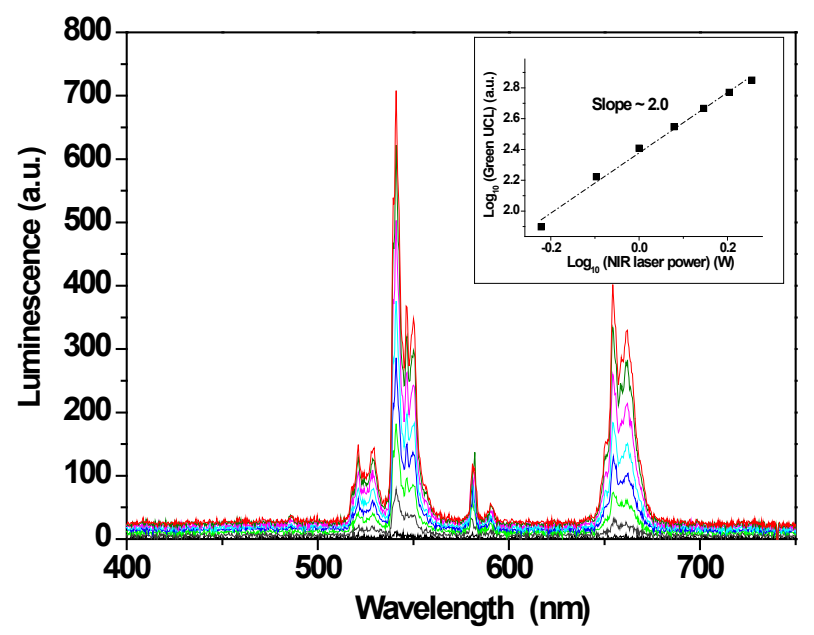

Figure 5. The evolution of the luminescence spectra acquired for the PS-based fibers containing upconversion nanophosphors (UCNPs) and rose bengal (RB) as a function of the NIR light excitation power $\left(\lambda_{\mathrm{ex}}=975 \mathrm{~nm}\right)$. Inset: the corresponding log-log plot of the green luminescence versus the excitation power.

The luminescence spectra shown in Figure 5 originate from the upconversion process and are characteristic for $\beta-\mathrm{NaYF}_{4}$-based UCNPs [37]. In particular, the slope of $\sim 2.0$ derived for the log-log plot of the green luminescence (integrated UCL bandwidths of the UCNPs at $525 \mathrm{~nm}$ and $545 \mathrm{~nm}$ ) versus the excitation power is similar to the slope values previously reported for the luminescence of $\beta-\mathrm{NaYF}_{4}$ UCNPs under NIR light excitation and points to the fact that the radiative transitions in UCNPs embedded in the PS matrix of the E-spun fibers follow the two-photon upconversion process (inset to Figure 5) [37,46].

A more detailed insight into the morphology of E-spun PSFs was gained by performing SEM and AFM imaging. The typical SEM images of PSFs are shown in Figure 6. As can be seen in this figure, the individual E-spun fibers reveal high structural quality and high porosity. Moreover, the accomplished SEM imaging and associated elemental analysis based on energy dispersive X-ray spectroscopy (EDX) of the E-spun fibers pointed to the presence of both UCNPs and SPIONs entrapped in the PS matrix (Figure 7). 


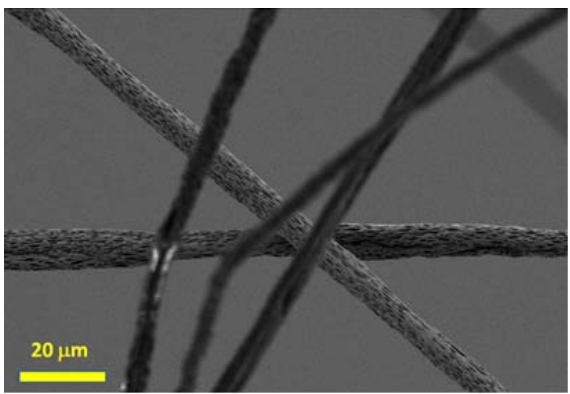

(a)

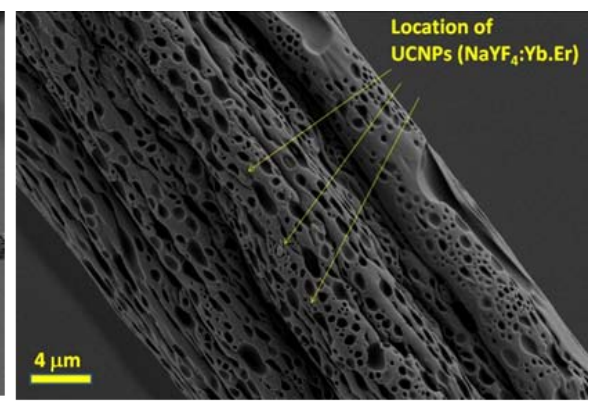

(b)

Figure 6. The morphology of the E-spun polystyrene-based fibers (PSFs) containing co-encapsulated UCNPs and SPIONs: (a) SEM image of the fiber network; (b) SEM image of an individual fiber.

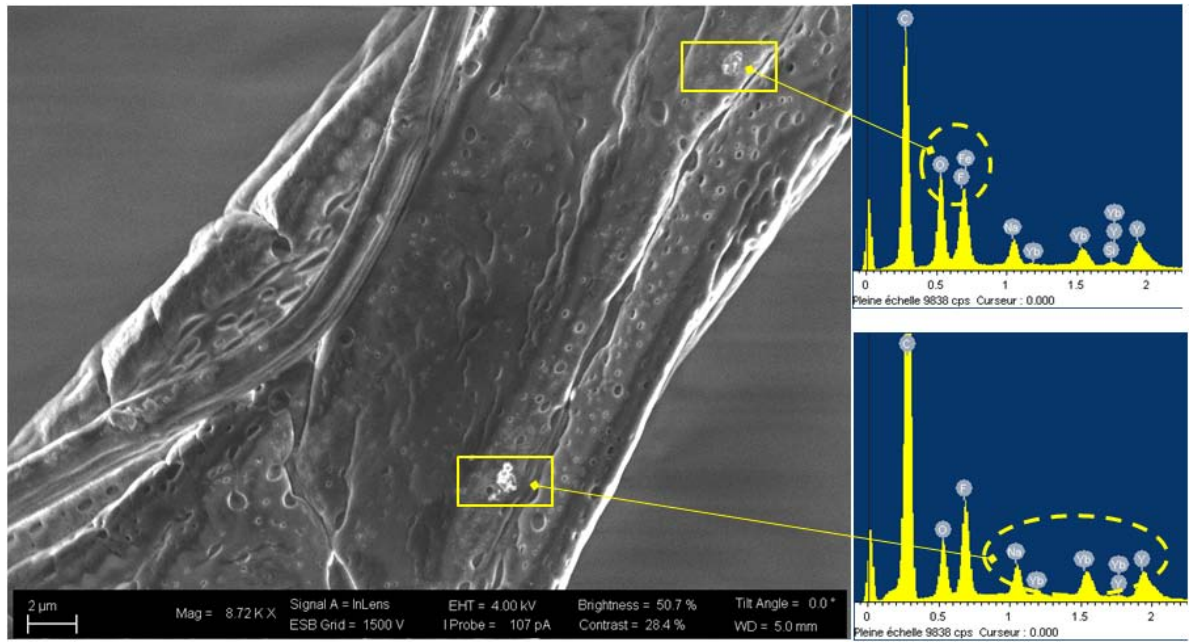

Figure 7. The SEM image of the surface of the E-spun PS fiber and the corresponding X-ray spectroscopy (EDX) spectra pointing to the presence of SPIONs (upper spectrum) and UCNPs (lower spectrum).

As can be seen in Figure 7, the typical EDX spectra acquired for E-spun PSFs revealed well-defined diffraction peaks characteristic for iron atoms in SPIONs and sodium, yttrium and ytterbium atoms in UCNPs.

The accomplished AFM imaging of individual fibers confirmed their high porosity, with an average pore depth of $c a$. 50-70 nm (Figure 8).

The magnetic properties of PS-based E-spun fibers loaded with UCNPs and SPIONs were checked in ESR experiments. Room temperature ESR measurements 
were performed for the original ferro-fluid containing the suspension of $\gamma-\mathrm{Fe}_{2} \mathrm{O}_{3}$ superparamagnetic particles (aqueous suspension, $10 \mathrm{mg}$ of Fe per $1 \mathrm{~mL}$, at $\mathrm{pH}$ 2) and for the E-spun fibers. The ESR spectra were acquired using a Bruker ESP300E spectrometer operating at the microwave frequency $\sim 9.7 \mathrm{GHz}$ (X-band). As can be seen in Figure 9, the ESR spectrum acquired for SPIONs embedded in the PS-based E-spun fibers broadens considerably as compared to the spectrum of SPIONs in the original ferro-fluid. The corresponding spectral line widths, $\Delta \mathrm{H}_{\mathrm{pp}}$, are of $\sim 600 \mathrm{G}$ and $\sim 1110 \mathrm{G}$, for SPIONs in ferro-fluid and PS fibers, respectively. This line-broadening can be ascribed to a stronger immobilization of $\gamma-\mathrm{Fe}_{2} \mathrm{O}_{3}$ superparamagnetic particles in large fragments of PS-based fibers. This observation is in good agreement with the previous reports on SPIONs immobilized in bulk polymer matrices [47].

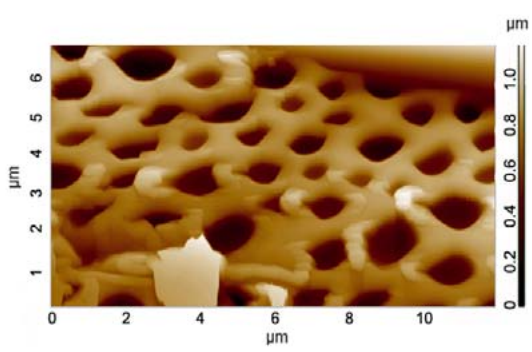

(a)

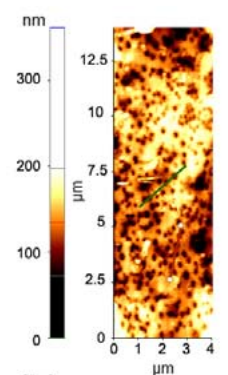

(b)

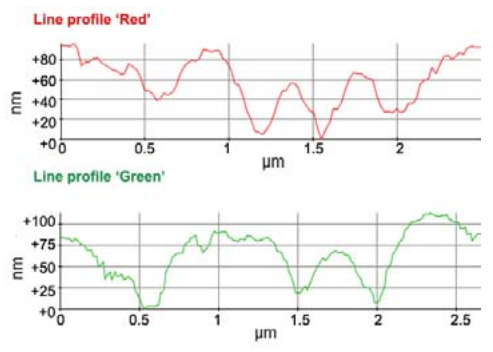

Figure 8. Atomic force microscopy (AFM) characterization of the PS-based E-spun fibers: AFM image of the surface of the E-spun fiber (a); and AFM-derived profiles of pores on the surface of the E-spun fiber (b).

The photodynamic action of the upconversion nanophosphors towards the generation of singlet oxygen under the excitation with NIR light was first verified for the aqueous suspensions of ground UCNPs. To generate ${ }^{1} \Delta_{\mathrm{g}}$ in aqueous suspensions of UCNPs, we employed a water-soluble ${ }^{1} \Delta_{\mathrm{g}}$-sensitizer, rose bengal (RB), from Sigma-Aldrich. The rationale of using RB as the ${ }^{1} \Delta_{g}$ photosensitizer in conjunction with UCNPs is explained in Figure 10. The example absorption spectrum of $13 \mu \mathrm{M}$ $\mathrm{RB}$ in $\mathrm{H}_{2} \mathrm{O}$ (pink trace in Figure 10) was measured with a UV-Vis spectrophotometer, Cary 50 Bio, Varian, Australia. The example UCL spectrum of UCNPs is also shown in this figure (green trace). As can be seen, the spectral absorption of RB perfectly matches the green emission region of UCNPs. Thus, this result confirms the rationale of using $\mathrm{RB}$ as the ${ }^{1} \Delta_{\mathrm{g}}$ photosensitizer in conjunction with $\beta-\mathrm{NaYF}_{4}: \mathrm{Yb}, \mathrm{Er}$ based UCNPs. 


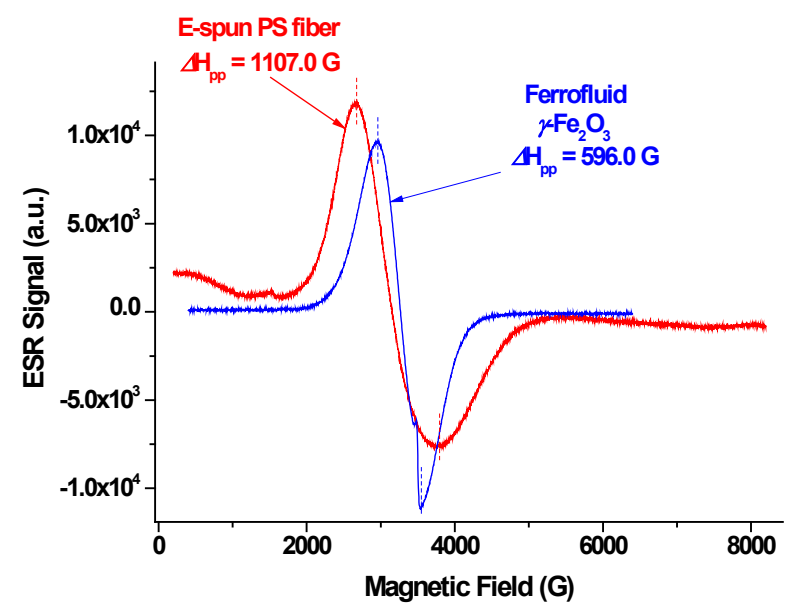

Figure 9. The comparison of the electron spin resonance (ESR) spectra acquired for SPIONS present in the original ferro-fluid (blue trace) and in the E-spun PS fibers loaded with UCNPs and SPIONs (red trace).

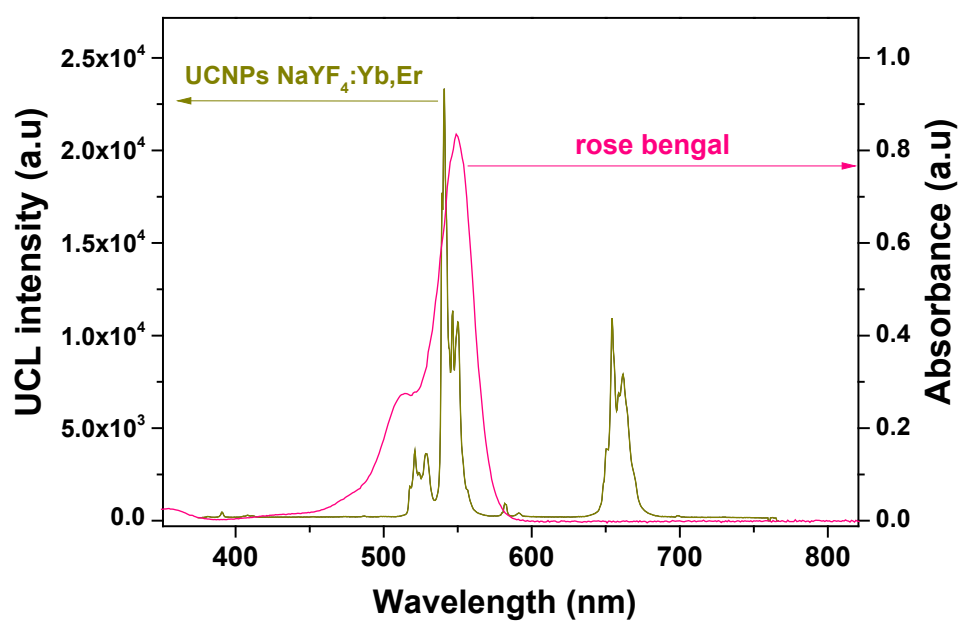

Figure 10. The overlapped spectra of the upconversion luminescence of UCNPs (green trace) and of the absorbance spectrum of $13-\mu \mathrm{M}$ water solution of rose bengal (pink trace). The UCNPs were suspended in water at a concentration of $0.5 \mathrm{mg} / \mathrm{mL}$.

The ESR technique was used to follow the formation of the photosensitized singlet oxygen in aqueous suspensions of UCNPs. The experiments were performed in deuterated water $\left(\mathrm{D}_{2} \mathrm{O}\right)$, which markedly enhances the singlet oxygen lifetime, from $3.1 \mu \mathrm{s}$ in $\mathrm{H}_{2} \mathrm{O}$ to $68 \mu \mathrm{s}$ in $\mathrm{D}_{2} \mathrm{O}$ [48]. The UCNPs were suspended in $\mathrm{D}_{2} \mathrm{O}$ by sonication. After sonication, the $\mathrm{D}_{2} \mathrm{O}$ suspensions 
of UCNPs were mixed with $\mathrm{D}_{2} \mathrm{O}$ solutions containing $\mathrm{RB}$ and a diamagnetic scavenger of singlet oxygen, 2,2,6,6-tetramethyl-4-piperidinol (TMP-OH). Upon reaction with singlet oxygen, TMP-OH converts to a stable nitroxyl radical, 4-hydroxy-2,2,6,6-tetramethylpiperidine-1-oxyl (TEMPOL) [49].

The final suspension contained $2 \mathrm{mg} / \mathrm{mL}$ of UCNPs, $50 \mu \mathrm{M}$ concentration of $\mathrm{RB}$ and $50 \mathrm{mM}$ concentration of TMP-OH. The 2-mL volume of this suspension was then transferred into a small Pyrex beaker $(5 \mathrm{~mL})$, which was positioned in the thermostated water bath (set to $25^{\circ} \mathrm{C}$ ) of the custom-designed photoreactor. To generate ${ }^{1} \Delta_{\mathrm{g}}$, the sample was exposed to NIR light from a 2-W NIR laser diode operating at $975 \mathrm{~nm}$ (MDL-III-975, GMP SA, Renens, Switzerland). The output of the NIR laser was positioned above the beaker, at a distance of $5 \mathrm{~cm}$ from the sample, thus providing the power density of $\sim 5.0 \mathrm{~W} / \mathrm{cm}^{2}$. During illumination with NIR light, the suspensions were magnetically stirred to prevent the precipitation of UCNPs. The experimental setup is shown in Figure 11.

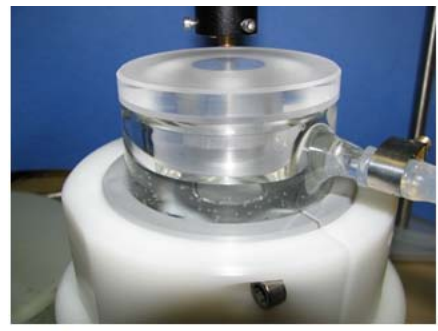

(a)

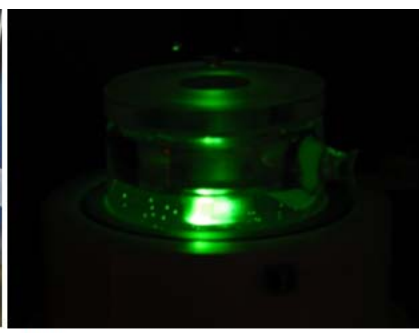

(b)

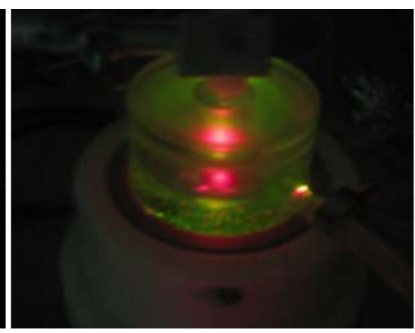

(c)

Figure 11. Photographs of the custom-made photoreactor for performing NIR light stimulated generation of singlet oxygen in aqueous suspensions of UCNPs: (a) visible light image; (b) image taken under illumination of the sample with NIR light in the absence of rose bengal; (c) image taken under illumination of the sample with NIR light in the presence of rose bengal.

After subsequent illumination steps, small aliquots of $\sim 15$ microliters were transferred into $0.7 \mathrm{~mm}$ inner diameter and $0.87 \mathrm{~mm}$ outer diameter glass capillary tubes (VitroCom, Mountain Lakes, NJ, USA), with a sample height of $\sim 40 \mathrm{~mm}$, and sealed on both ends with a tube sealing compound (Cha-seal from Chase Scientific Glass, Rockwood, TN, USA) for performing ESR measurements. To maximize the sample volume in the active zone of the ESR cavity, assemblies of seven tightly packed capillaries were bundled together and inserted into the wide-bore quartz capillary (standard ESR quartz tube with $2.9 \mathrm{~mm}$ ID and $4 \mathrm{~mm}$ OD, Model 707-SQ-250M, from Wilmad-LabGlass Inc., Vineland, NJ, USA). Such a setup resulted in a $\sim 65$ microliter sample volume in the active zone of the rectangular $\mathrm{TE}_{102}$ cavity. 
As can be seen in Figure 11c, under NIR light excitation, the bright yellowish green luminescence, which is typical for $\mathrm{NaYF}_{4}: \mathrm{Yb}$,Er nanophosphors, changes its color, due to a partial absorption of the green UCL by the photosensitizer (RB). This confirms the energy transfer from the NIR light excited UCNPs to RB.

The ESR results of singlet oxygen detection under NIR light excitation of aqueous suspensions containing UCNPs and the ${ }^{1} \Delta_{\mathrm{g}}$-sensitizer, RB, are shown in Figure 12.

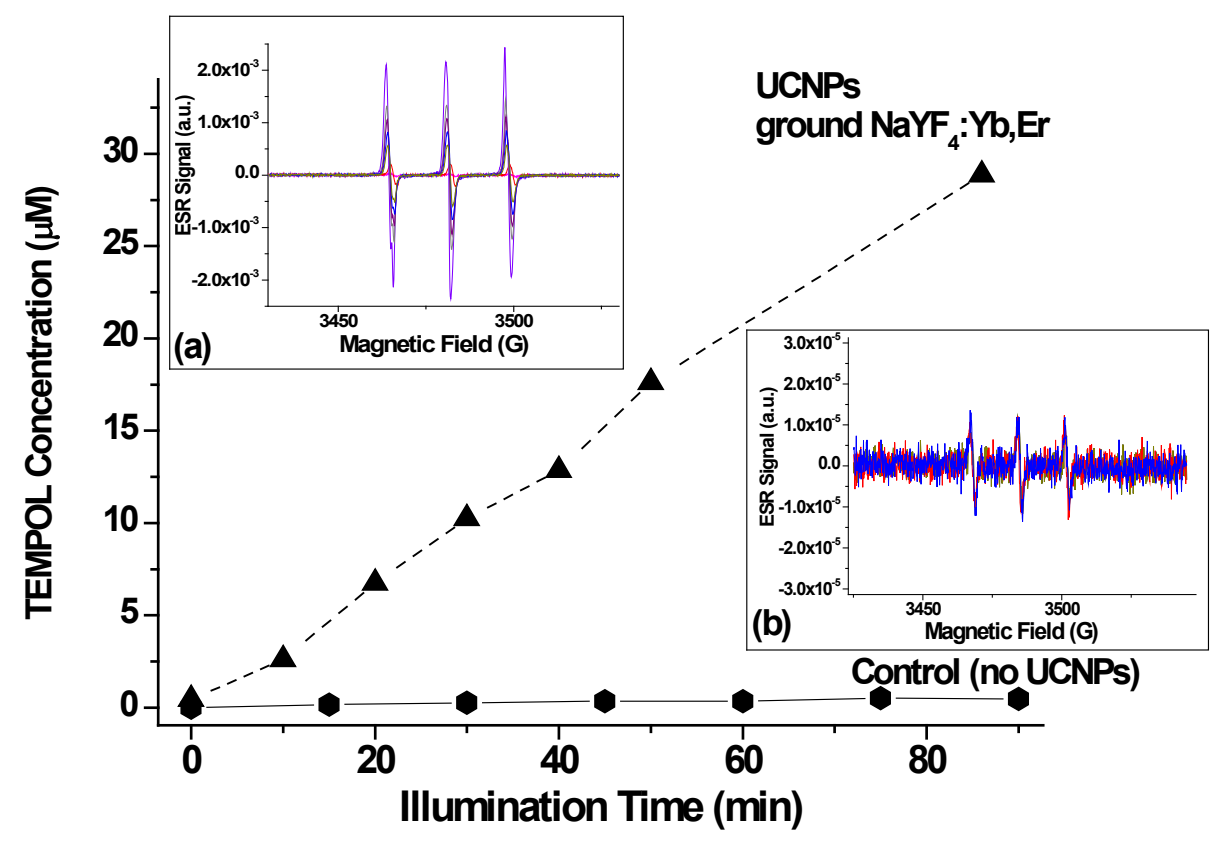

Figure 12. The photodynamic process of RB-mediated singlet oxygen generation under NIR light excitation of the $\mathrm{D}_{2} \mathrm{O}$ suspension of UCNPs (black triangles) and the control trace for the $\mathrm{D}_{2} \mathrm{O}$ solution of $\mathrm{RB}$ (black hexagons). Insets: (a) typical time evolution of ESR signals of 4-hydroxy-2,2,6,6-tetramethylpiperidine-1-oxyl (TEMPOL) during NIR light illumination of the aqueous suspension of UCNPs in the presence of RB; (b) time evolution of ESR signals of TEMPOL during the control measurements of the $\mathrm{D}_{2} \mathrm{O}$ solution of $\mathrm{RB}$ under NIR light excitation (in the absence of UCNPs). In both cases, the concentrations of RB and 2,2,6,6-tetramethyl-4-piperidinol (TMP-OH) were of $50 \mu \mathrm{M}$ and $50 \mathrm{mM}$, respectively.

As can be seen in Figure 12, under exposure to NIR light for $~ 90$ min, marked concentrations (tens of micromoles) of ${ }^{1} \Delta_{\mathrm{g}}$ were generated in the aqueous suspension containing UCNPs and the ${ }^{1} \Delta_{\mathrm{g}}$-sensitizer, RB. 
Finally, we implemented a similar approach to verify by ESR the photodynamic efficiency of the PS-based E-spun fibers towards the generation of singlet oxygen under NIR light illumination in aqueous media. Prior to performing the ESR detection of NIR-light excited singlet oxygen, a small bundle of E-spun PS fibers was inserted into a thin glass capillary (0.7 $\mathrm{mm}$ ID and $0.87 \mathrm{~mm}$ OD). Subsequently, the capillary was filled with $50 \mathrm{mM}$ solution of TMP-OH in $\mathrm{D}_{2} \mathrm{O}$, sealed on both ends with Cha-seal and inserted into $4.0 \mathrm{~mm}$ OD quartz tube. Then, NIR illumination was performed using a 2-W laser diode operating at $975 \mathrm{~nm}$. After each illumination step, the capillary was positioned in the spectrometer's cavity, and standard field-swept ESR spectra were acquired. The experimental setup is shown in Figure 13.

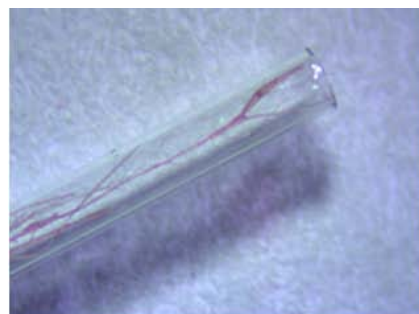

(a)

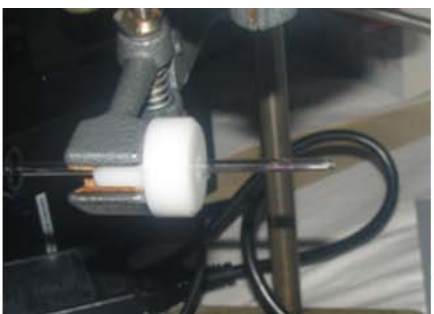

(b)

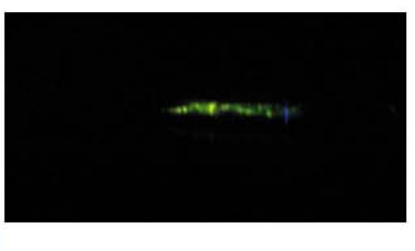

(c)

Figure 13. The experimental setup for performing NIR light stimulated the generation of singlet oxygen in the presence of PS-based E-spun fibers immersed in $\mathrm{D}_{2} \mathrm{O}$. (a) The photograph of the termination of the $0.7 \mathrm{~mm}$ inner diameter glass capillary loaded with PS-based fibers and prepared to be filled with the $\mathrm{D}_{2} \mathrm{O}$ solution of TMP-OH; (b) the photograph of the $4.0 \mathrm{~mm}$ outer diameter quartz capillary containing the $0.7 \mathrm{~mm}$ inner diameter glass capillary with PS-based fibers immersed in the $\mathrm{D}_{2} \mathrm{O}$ solution of TMP-OH and positioned under the output of 2-W NIR laser diode $\left(\lambda_{\mathrm{ex}}=975 \mathrm{~nm}\right)$; $(\mathrm{c})$ the photograph showing the luminescence of PS-based fibers positioned in the $0.7 \mathrm{~mm}$ inner diameter glass capillary and exposed to NIR light: the yellowish green UCL of UCNPs embedded in polystyrene fibers can be seen.

Thus, as can be seen in Figure 14, micromolar concentrations of singlet oxygen were photo-sensitized during NIR light illumination for ca. $350 \mathrm{~min}$ of PS-based E-spun fibers containing UCNPs, SPIONs and RB. It is worth noting that this result was obtained for a very small total mass of the PS fibers $(\sim 0.2 \mathrm{mg})$ in an aqueous milieu of $\sim 5-6 \mu \mathrm{L}$. We associate this photodynamic activity of the PS-based E-spun fibers with their high porosity and large surface-area-to-volume ratio. The marked slope of the ESR spectra acquired for TEMPOL, which can be seen in inset (a) to Figure 14, is due to a relatively large background signal related to SPIONs entrapped in the E-spun fibers (depicted by the red trace in Figure 9). 


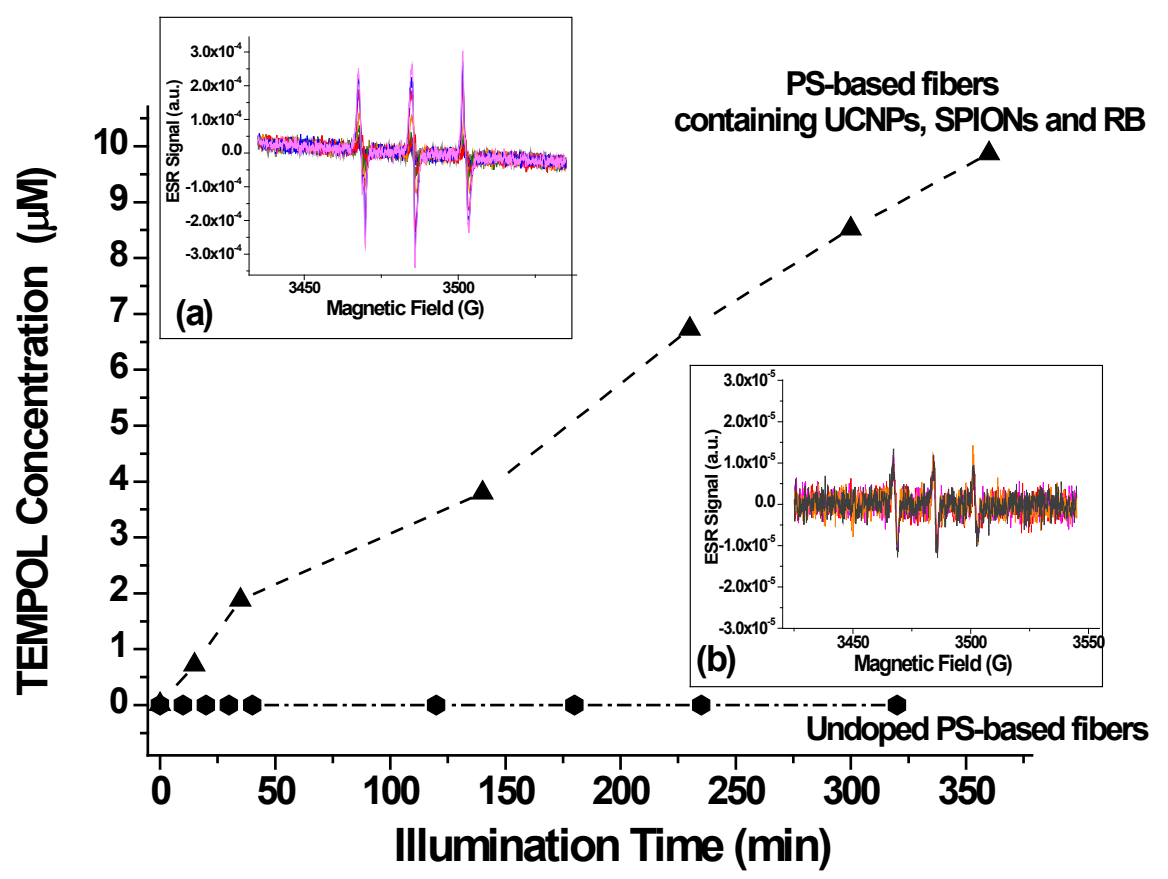

Figure 14. The ESR assay of the photodynamic formation of singlet oxygen in $\mathrm{D}_{2} \mathrm{O}$ under the NIR light excitation of PS-based E-spun fibers loaded with UCNPs, SPIONs and RB (black triangles) and the control trace acquired for the undoped PS-based E-spun fibers (black hexagons). In both cases, the PS-based fibers were immersed in $50 \mathrm{mM}$ solution of TMP-OH in $\mathrm{D}_{2} \mathrm{O}$. Inset: (a) the time evolution of the ESR signals of TEMPOL under NIR light illumination of PS-based E-spun fibers loaded with UCNPs, SPIONs and RB; (b) the time evolution of the ESR signals of TEMPOL under NIR light illumination of the undoped PS-based E-spun fibers.

\section{Conclusions}

We successfully prepared PS-based multi-functional magneticphotoluminescent-photocatalytic micro- and nano-fibers by a single-step ES process. The fabrication technique was simple, and the materials used were inexpensive. Due to the concomitant loading with UCNPs and RB, the E-spun PS-based fibers revealed photocatalytic activity on exposure to NIR light. In particular, under NIR light excitation, the UCNPs embedded in PS-based fibers emitted the upconversion luminescence (UCL) in the visible range, thus enabling the photosensitization of singlet oxygen by RB. Moreover, due to the doping with $\gamma-\mathrm{Fe}_{2} \mathrm{O}_{3}$ SPIONs, these nanostructured PS-based fibers revealed also magnetic properties, typical for the systems containing iron oxide superparamagnetic nanoparticles. 
Acknowledgments: The authors would like to acknowledge the financial support of the Swiss National Science Foundation (SNSF) through the Nano-Tera.ch Focused Project (NTF), "NanoUp". This work was also partly supported by the Swiss National Science Foundation (SNSF) grant no. PP00P2_123373, the University of Fribourg, and the Adolphe Merkle Foundation (including FriMat). We are grateful to Paul Bowen (Ecole Polytechnique Fédérale de Lausanne) for milling the UCNPs used in this work.

Conflicts of Interest: The authors declare no conflict of interests.

\section{References}

1. Ladja, R.; Bitar, A.; Eissa, M.M.; Fessi, H.; Mugnier, Y.; Le Dantec, R.; Elaissari, A. Polymer encapsulation of inorganic nanoparticles for biomedical applications. Int. J. Pharm. 2013, 458, 230-241.

2. Peng, W.; Qu, S.; Cong, G.; Wang, Z. Synthesis and structures of morphology-controlled ZnO nano- and microcrystals. Cryst. Growth Des. 2006, 6, 1518-1522.

3. Chatterjee, J.; Bettge, M.; Haik, Y.; Jen Chen, C. Synthesis and characteriza-tion of polymer encapsulated $\mathrm{Cu}-\mathrm{Ni}$ magnetic nanoparticles for hyperthermia applications. J. Magn. Magn. Mater. 2005, 293, 303-309.

4. Hirai, T.; Saito, T.; Komasawa, I. Stabilization of CdS nanoparticles immobilized on thiol-modified polystyrene particles by encapsulation with polythiourethane. J. Phys. Chem. B 2001, 105, 9711-9714.

5. Zeng, Z.; Yu, J.; Guo, Z.-X. Preparation of functionalized core-shell alu-mina/polystyrene composite nanoparticles, 1. Macromol. Chem. Phys. 2005, 206, 1558-1567.

6. Sondi, I.; Fedynyshyn, T.H.; Sinta, R.; Matijevic, E. Encapsulation of nanosizedsilica by in situ polymerization of tert-butyl acrylate monomer. Langmuir 2000, 16, 9031-9034.

7. Venugopal, J.; Ramakrishna, S. Applications of polymer nanofibers in biomedicine and biotechnology. Appl. Biochem. Biotechnol. 2005, 125, 147-157.

8. Khetani, S.R.; Bhatia, S.N. Engineering tissues for in vitro applications. Curr. Opin. Biotechnol. 2005, 17, 524-553.

9. Ravichandran, R.; Gandhi, S.; Sundaramurthi, D.; Sethuraman, S.; Krishnan, U.M. Hierarchical mesoporous silica nanofibers as multifunctional scaffolds for bone tissue regeneration. J. Biomater. Sci. Polym. Ed. 2013, 24, 1988-2005.

10. Focarete, M.L.; Gualandi, C. Potentialities of electrospun polymeric nanofibres in the biomedical field. J. Tissue Sci. Eng. 2013, 4, 1-4.

11. Doshi, J.; Reneker, D.H. Electrospinning process and applications of electrospun fibers. J. Electrost. 1995, 35, 151-160.

12. Huang, Z.-M.; Zhang, Y.-Z.; Kotaki, M.; Ramakrishna, S. A review on polymer nanofibers by electrospinning and their applications in nanocomposites. Compos. Sci. Technol. 2003, $63,2223-2253$.

13. Oraby, M.A.; Waley, A.I.; El-Dewany, A.I.; Saad, E.A.; Abd El-Hady, B.M. Electrospinning of gelatin functionalized with silver nanoparticles for nanofiber fabrication. Model. Numer. Simul. Mater. Sci. 2013, 3, 95-105. 
14. El-Assar, M.R.; Fouda, M.M.G.; Kenawy, E.-R. Electrospinning of functionalized copolymer nanofibers from poly(acrylonitrile-co-methyl methacrylate). Adv. Polym. Technol. 2013, 32, 1-11.

15. Sill, T.J.; von Recum, H.A. Electrospinning: Applications in drug delivery and tissue engineering. Biomaterials 2008, 29, 1989-2006.

16. Teo, W.-E.; Inai, R.; Ramakrishna, S. Technological advances in electrospinning of nanofibers. Sci. Technol. Adv. Mater. 2011, 12, 013002:1-013002:19.

17. Kim, T.-G.; Ragupathy, D.; Gopalan, A.I.; Lee, K.-P. Electrospun carbon nanotubes-gold nanoparticles embedded nanowebs: Prosperous multi-functional nanomaterials. Nanotechnology 2010, 21, 134021:1-134021:11.

18. Mosinger, J.; Lang, K.; Kubat, P.; Sykora, J.; Hof, M.; Plistil, L.; Mosinger, B. Photofunctional polyurethane nanofabrics doped by zinc tetraphenylporphyrin and zinc phthalocyanine photosensitizers. J. Fluoresc. 2009, 19, 705-713.

19. Mosinger, J.; Lang, K.; Hostomsky, J.; Franc, J.; Sykora, J.; Hof, M.; Kubat, P. Singlet oxygen imaging in polymeric nanofibers by delayed fluorescence. J. Phys. Chem. B 2010, 114, 15773-15779.

20. Mosinger, J.; Lang, K.; Plistil, L.; Josenska, S.; Hostomsky, J.; Zelinger, Z.; Kubat, P. Fluorescent polyurethane nanofabrics: A source of singlet oxygen and oxygen sensing. Langmuir 2010, 26, 10050-10056.

21. Li, C.; Lin, J. Rare earth fluoride nano-/microcrystals: Synthesis, surface modification and application. J. Mater. Chem. 2010, 20, 6831-6847.

22. Wang, F.; Liu, X. Recent advances in the chemistry of lanthanide-doped upconversion nanocrystals. Chem. Soc. Rev. 2009, 38, 976-989.

23. Chatterjee, D.K.; Rufalhah, A.J.; Zhang, Y. Upconversion fluorescence imaging of cells and small animals using lanthanide doped nanocrystals. Biomaterials 2008, 29, 937-943.

24. Wang, S.; Feng, J.; Song, S.; Zhang, H. Rare earth fluorides upconversion nanophosphors: From synthesis to applications in bioimaging. CrystEngComm 2013, 15, 7142-7151.

25. Haase, M.; Schafer, H. Upconverting nanoparticles. Angew. Chem. Int. Ed. 2011, 50, 5808-5829.

26. Boyer, J.C.; van Veggel, F.C. Absolute quantum yield measurements of colloidal $\mathrm{NaYF}_{4}$ : $\mathrm{Er}^{3+}, \mathrm{Yb}^{3+}$ upconverting nanoparticles. Nanoscale 2010, 2, 1417-1419.

27. Chen, G.; Qiu, H.; Fan, R.; Hao, S.; Tan, S.; Yang, C.; Han, G. Lanthanide-doped ultrasmall yttrium fluoride nanoparticles with enhanced multicolor upconversion photoluminescence. J. Mater. Chem. 2012, 22, 20190-20196.

28. Chen, G.; Shen, J.; Ohulchanskyy, T.Y.; Patel, N.J.; Kutikov, A.; Li, Z.; Song, J.; Pandey, R.K.; Ågren, H.; Prasad, P.N.; et al. $\left(\alpha-\mathrm{NaYbF}_{4}: \mathrm{Tm}^{3+}\right) / \mathrm{CaF}_{2}$ core/shell nanoparticles with efficient near-infrared to near-infrared upconversion for high-contrast deep tissue bioimaging. ACS Nano 2012, 6, 8280-8287.

29. Ostrowski, A.D.; Chan, E.M.; Gargas, D.J.; Katz, E.M.; Han, G.; Schuck, J.; Milliron, D.J.; Cohen, B.E. Controlled synthesis and single-particle imaging of bright, sub-10 nm lanthanide-doped upconverting nanocrystals. ACS Nano 2012, 6, 2686-2692. 
30. Shen, J.; Chen, G.; Ohulchanskyy, T.Y.; Kesseli, S.J.; Buchholz, S.; Li, Z.; Prasad, P.N.; Han, G. Tunable near infrared to ultraviolet upconversion luminescence enhancement in $\left(\alpha-\mathrm{NaYF}_{4}: \mathrm{Yb}, \mathrm{Tm}\right) / \mathrm{CaF}_{2}$ core/shell nanoparticles for in situ real-time recorded biocompatible photoactivation. Small 2013, 9, 3213-3217.

31. Colombo, M.; Carregal-Romero, S.; Casula, M.F.; Gutierrez, L.; Morales, M.P.; Boehm, I.B.; Heverhagen, J.T.; Prosperi, D.; Parak, W.J. Biological applications of magnetic nanoparticles. Chem. Soc. Rev. 2012, 41, 4306-4334.

32. Laurent, S.; Forge, D.; Port, M.; Roch, A.; Robic, C.; Elst, L.V.; Muller, R.N. Magnetic iron oxide nanoparticles: Synthesis, stabilization, vectorization, physicochemical characterizations, and biological applications. Chem. Rev. 2008, 108, 2064-2110.

33. Zhang, D.; Karki, A.B.; Rutman, D.; Young, D.P.; Wang, A.; Cocke, D.; Ho, T.H.; Guo, Z. Electrospun polyacrylonitrile nanocomposite fibers reinforced with $\mathrm{Fe}_{3} \mathrm{O}_{4}$ nanoparticles: Fabrication and property analysis. Polymer 2009, 50, 4189-4198.

34. Zhu, J.; Wei, S.; Rutman, D.; Haldolaarachchige, N.; Young, D.P.; Guo, Z. Magnetic polyacrylonitrile-Fe@FeO nanocomposite fibers-electrospinning, stabilization and carbonization. Polymer 2011, 52, 2947-2955.

35. De Santis, R.; Gloria, A.; Russo, T.; D’Amora, U.; Zeppetelli, S.; Dionigi, C.; Sytcheva, A.; Herrmannsdörfer, T.; Dediu, V.; Ambrosio, L. A basic approach toward the development of nanocomposite magnetic scaffolds for advanced bone tissue engineering. J. Appl. Polym. Sci. 2011, 122, 3599-3605.

36. Meng, J.; Xiao, B.; Zhang, Y.; Liu, J.; Xue, H.; Lei, J.; Kong, H.; Huang, Y.; Jin, Z.; Gu, N.; et al. Super-paramagnetic responsive nanofibrous scaffolds under static magnetic field enhance osteogenesis for bone repair in vivo. Nat. Sci. Rep. 2013, 3, 2655.

37. Suyver, J.F.; Grimm, J.; van Veen, M.K.; Biner, D.; Kramer, K.W.; Gudel, H.U. Upconversion spectroscopy and properties of $\mathrm{NaYF}_{4}$ doped with $\mathrm{Er}^{3+}, \mathrm{Tm}^{3+}$ and/or $\mathrm{Yb}^{3+}$. J. Lumin. 2006, 117, 1-12.

38. Ye, X.C.; Collins, J.E.; Kang, Y.J.; Chen, J.; Chen, D.T.N.; Yodh, A.G.; Murray, C.B. Morphologically controlled synthesis of colloidal upconversion nanophosphors and their shape-directed self-assembly. Proc. Natl. Acad. Sci. USA 2010, 107, 22430-22435.

39. Gryglik, D.; Lach, M.; Miller, J.S. The aqueous photosensitized degradation of butylparaben. Photochem. Photobiol. Sci. 2009, 8, 549-555.

40. DeRosa, M.C.; Crutchley, R.J. Photosensitized singlet oxygen and its applications. Coord. Chem. Rev. 2002, 233-234, 351-371.

41. Liu, X.; Zhang, Y.; Tu, L.; Wang, Y.; Zeng, Q.; Li, C.; Shi, Z.; Kong, X.; Zhang, H. Breakthrough in concentration quenching threshold of upconversion luminescence via spatial separation of emitter doping area for Bio-application. Chem. Commun. 2011, 47, 11957-11959.

42. Soukka, T.; Kuningas, K.; Rantanen, T.; Haaslahti, V.; Lovgren, T. Photochemical characterizationof up-converting inorganic lanthanide phosphors as potential labels. J. Fluoresc. 2005, 15, 513-528.

43. Bee, A.; Massart, R.; Neveau, S. Synthesis of very fine maghemite particles. J. Magn. Magn. Mater. 1995, 149, 6-9. 
44. Van Ewijk, G.A.; Vroege, G.J.; Philipse, A.P. Convenient preparation methods for magnetic colloids. J. Magn. Magn. Mater. 1999, 201, 31-33.

45. Chastellain, M.; Petri, A.; Hofmann, H. Particle size investigations of a multistep synthesis of PVA coated superparamagnetic nanoparticles. J. Colloid Interface Sci. 2004, 278, 353-360.

46. Lim, S.F.; Ryu, W.S.; Austin, R.H. Particle size dependence of the dynamic photophysical properties of $\mathrm{NaYF}_{4}: \mathrm{Yb}$, Er nanocrystals. Opt. Express 2010, 18, 2309-2316.

47. Noginova, N.; Chen, F.; Weaver, T.; Giannelis, E.P.; Bourlinos, A.B.; Atsarkin, V.A. Magnetic resonance in nanoparticles: Between ferro- and paramagnetism. J. Phys.: Condens. Matter 2007, 19, 246208:1-246208:15.

48. Schweitzer, C.; Schmidt, R. Physical mechanisms of generation and deactivation of singlet oxygen. Chem. Rev. 2003, 103, 1685-757.

49. Sienkiewicz, A.; Vileno, B.; Pierzchala, K.; Czuba, M.; Marcoux, P.; Graczyk, A.; Fajer, P.G.; Forro, L. Oxidative stress-mediated protein conformation changes: ESR study of spin-labelled staphylococcal nuclease. J. Phys.: Condens. Matter 2007, 19, 285201:1-285201:13. 


\title{
Release of Carbon Nanotubes from Polymer Nanocomposites
}

\author{
Lukas Schlagenhauf, Frank Nüesch and Jing Wang
}

\begin{abstract}
Carbon nanotube (CNT)/polymer nanocomposites have superior properties compared to the neat polymer matrix. They now are widely used in industry, but questions have been raised about the risks of such materials since CNTs can be toxic when inhaled. For a risk assessment of CNT nanocomposites, it is crucial to know whether CNTs from nanocomposites can be released into the environment or if they remain embedded in the matrix. This review article summarizes the studies that investigated the release of CNTs from nanocomposites during the service life. Three scenarios are reviewed, the release of particles due to mechanical impact, the release due to weathering processes, and the release due to fire. A release during composite production and disposal is not incorporated.
\end{abstract}

Reprinted from Fibers. Cite as: Schlagenhauf, L.; Nüesch, F.; Wang, J. Release of Carbon Nanotubes from Polymer Nanocomposites. Fibers 2014, 2, 108-127.

\section{Introduction}

Carbon nanotubes (CNTs) exhibit unique mechanical, electrical, and optical properties, therefore they have been considered as a nanofiller for composites. CNT nanocomposites have superior or additional properties compared to their neat matrix materials. These include mechanical properties such as tensile strength and Young's modulus [1,2], energy absorption [3], improved scratch and wear resistance [4], electrical and thermal conductivity [5,6], fire resistance [7], and optical properties [8]. Nowadays, CNT nanocomposites are widely used for a variety of applications in different industries, e.g., for automotive, aerospace, defense, electronics, energy, and sporting goods [9].

Because of the extensive usage, the question has been raised whether these nanocomposites pose a threat to the human health and the environment since it is already known, that CNTs can cause health problems. They can cause oxidative stress, genotoxicity, inflammation, and also fibrosis [10-16].

During the life cycle of a CNT nanocomposite, different scenarios can be identified where CNTs might be released into the environment and pose a threat. Those scenarios have already been developed in several publications [9,17-23]. In general, the life cycle can be divided into three stages where exposure to humans can occur, production and processing, service life, and disposal. The purpose of this paper is to review the scientific work up to date that investigated 
the potential of release of CNTs into the environment. It concentrates on the possible release during the service life where untrained humans are in contact with nanocomposites. Three possible pathways are considered, particle release due to mechanical impact, degradation of the matrix due to weathering processes, and release due to fire incidents.

A summary of all used abbreviations in this review is presented in Table 1.

Table 1. Abbreviations.

\begin{tabular}{ll}
\hline ABS & Acrylonitrile butadiene styrene \\
ATR-FTIR & Attenuated total reflectance-fourier transform infrared spectroscopy \\
AUC & Analytical ultracentrifugation \\
CNF & Carbon nanofiber \\
CNT & Carbon nanotube \\
CRP & Carbon fiber reinforced plastic \\
EVA & Ethylene-vinyl acetate \\
PA6 & Polyamide 6, Nylon 6 \\
PC & Polycarbonate \\
PE & Polyethylene \\
PEN & Polyethylene naphthalate \\
PMMA & Poly(methyl methacrylate) \\
POM & Polyoxymethylene \\
PP & Polypropylene \\
PS & Polystyrene \\
PU & Polyurethane \\
SEM & Scanning electron microscope \\
SMPS & Scanning mobility particle sizer \\
TEM & Transmission electron microscopy \\
\hline
\end{tabular}

\section{Results of Release Studies}

\subsection{Release of Particles Due to Mechanical Impact}

From nanocomposites, particles can be released due to several mechanical actions. Those involve scratching, sanding, sawing, and drilling. So far, no standard method has been established to simulate these actions and also no model has been established to compare them. To measure the release of particles during the lifespan of a consumer product, the Taber Abraser can be used. It is a widely used device to simulate sanding processes and to study abrasion resistances of materials and coatings with its own international standards (e.g., ISO 7784-2:1997; ISO 9352:1995; ISO 5470-1:1999; ASTM G195-08). It provides a continuous 
abrasion process under defined conditions that can be repeated by any other laboratory. Several studies have already used the Taber Abraser to generate particles from nanocomposites, to measure the particle size distribution, and to search for released nanoparticles [24-28].

Other studies simulate sanding process directly with sanding machines and other workshop tools. Those measurements are closer to the reality for processes with high shear forces but it is more complicated to measure the particle size distributions because it can happen that the sanding device is producing nanoparticles itself $[29,30]$, or that big differences in the particle concentrations can appear either because the particle concentration depends on the applied pressure, or due to burst events [28]. To achieve repeatable results for real sanding studies, self-made measurement devices have to be established as it has been done e.g., by Göhler et al. [31].

For the characterization of the released particles, several measurement devices are available. A review on that subject has been done already by Kuhlbusch et al. [32]. Generally, it is expected that an abrasion experiment releases particles in the size range from a few nanometers to several micrometers. A determination for the full size range has been done e.g., by Wohlleben et al. [28]. They used Fraunhofer diffraction and measured particles with diameters up to $200 \mu \mathrm{m}$ for a PU nanocomposite. To measure the release of individual free standing CNTs, measurement devices that can detect and collect particles in the nanosize are preferred.

The reviewed papers of this section are summarized in Table 2.

The first study that investigated the release of nanofibers from a nanocomposite was done by Mazzuckelli et al. [33]. They measured the release of particles and fibers from a carbon nanofiber (CNF) composite during different stages of the production process including wet sawing. Particle concentration measurements during weighing and mixing of CNFs in an unventilated area and also during wet-saw cutting of the composite revealed a release of airborne particles. Further, filter sampling according to NIOSH Manual of Analytical Methods (NMAM) method 5040 showed that also CNFs have been released during those treatments.

Bello et al. [34-36] have published several studies with applied wet and dry drilling, band-sawing under dry conditions, and sawing with a rotary cutting wheel under wet conditions on nanocomposites. The investigated samples consisted either of a carbon fiber/epoxy composite with aligned CNTs placed at the center ply interface or a woven alumina fiber cloth with aligned CNTs grown on the surface of the fibers, impregnated with an epoxy. During the processing of the composites, the aerosol particle concentration and particle size distribution in the nano- and microrange was measured. Further, particles were collected with an asbestos sampling cassette for fiber analysis and also TEM samples have been produced. 
Table 2. Carbon Nanotube (CNT) release studies - Release of particles due to mechanical impact.

\begin{tabular}{|c|c|c|c|}
\hline Study & Material & Particle Generation Method & Nanofiller release? \\
\hline Mazzuckelli et al. [33] & $\mathrm{CNF} /$ polymer composite & $\begin{array}{l}\text { Composite preparation } \\
\text { and wet sawing }\end{array}$ & $\begin{array}{l}\text { Free standing CNFs collected on filter } \\
\text { after CNF weighing and wet sawing }\end{array}$ \\
\hline Bello et al. [34-36] & $\begin{array}{l}\text { Microfiber (carbon or } \\
\text { alumina)/CNT/epoxy } \\
\text { composite }\end{array}$ & $\begin{array}{l}\text { Wet and dry drilling, } \\
\text { band-sawing under dry } \\
\text { conditions, and sawing } \\
\text { with a rotary cutting wheel } \\
\text { under wet conditions }\end{array}$ & $\begin{array}{l}\text { Submicron and sharp fibers found } \\
\text { for all samples, release of CNT } \\
\text { agglomerates only for drilling }\end{array}$ \\
\hline Cena and Peters [37] & CNT/epoxy composite & Sanding & $\begin{array}{l}\text { Release of nanosized particles with } \\
\text { irregular shapes and protruded } \\
\text { CNTs, no free standing CNTs found }\end{array}$ \\
\hline Wohlleben et al. $[25,28]$ & $\begin{array}{l}\text { CNT/POM and } \\
\text { CNT/PU composites }\end{array}$ & Sanding and Taber Abraser & No nanofiller release \\
\hline Methner et al. [38] & CNF/epoxy composite & $\begin{array}{l}\text { Wet sawing, surface } \\
\text { grinding, and belt sawing }\end{array}$ & $\begin{array}{l}\text { Free standing CNFs found in the } \\
\text { process area and at the personal } \\
\text { breathing zone }\end{array}$ \\
\hline Ogura et al. [39] & SWCNT/PP composite & Microgrinder & $\begin{array}{l}\text { Particles with protruding SWCNTs, } \\
\text { no release of free standing SWCNTs }\end{array}$ \\
\hline Golanski et al. [40] & $\begin{array}{l}\mathrm{CNT} / \mathrm{PC} \text { and } \\
\mathrm{CNT} / \mathrm{PA} 6 \text { composites }\end{array}$ & Rake and metallic brush & $\begin{array}{l}\text { No release for the rake, release of } \\
\text { CNTs by metallic brush only when } \\
\text { CNTs are poorly distributed in the } \\
\text { polymer matrix }\end{array}$ \\
\hline Schlagenhauf et al. [27] & CNT/epoxy composite & Taber Abraser & $\begin{array}{l}\text { Release of CNTs (average length } \approx \\
304 \mathrm{~nm} \text { ) }\end{array}$ \\
\hline Hellmann et al. [41] & CNT/epoxy composite & Sanding & $\begin{array}{l}\text { Particles with protruding CNTs, } \\
\text { no release of free standing CNTs }\end{array}$ \\
\hline Huang et al. [42] & CNT/epoxy composite & Sanding & $\begin{array}{l}\text { No release of CNTs except for } \\
\text { a } 4 \mathrm{wt} \% \text { CNT sample }\end{array}$ \\
\hline
\end{tabular}

The aerosol measurements showed that drilling releases more particles with a broader particle size distribution than sawing, this is partially caused by smoke that is generated during drilling. TEM analysis of collected particles show that respirable fibers are released during all processes, but single CNTs have not been found. In contrast to sawing, drilling caused a release of CNT agglomerates [36].

Cena and Peters [37] investigated the particle release during weighing of CNTs and sanding of a $2 \mathrm{wt} \% \mathrm{CNT} /$ epoxy composite. Only few airborne nanoparticles were measured during the two actions. Images from TEM imaging show micrometer sized particles with protruding CNTs. The authors conclude that as long as the toxicity of such particles is unknown, precautions should be applied to avoid exposures to workmen. Further, they found out that a biological safety cabinet was more effective than a custom fume hood to avoid exposures. 
A POM/CNT nanocomposite was investigated by Wohlleben et al. [25]. Abrasion was applied by the Taber Abraser or by a sanding machine. The particle size distribution of abraded particles was measured by AUC (sanding machine) and by SMPS (Taber Abraser, only for airborne particles). The AUC data shows that particles $>2.7 \mu \mathrm{m}$ represent more than $99 \mathrm{wt} \%$ of the released particles. According to the SMPS data, $80 \%$ of the measured particles in the nanorange are smaller than $100 \mathrm{~nm}$, but no influence of the nanofiller on the particle size distribution could be detected neither by AUC nor by SMPS. Further, no release of CNTs due to abrasion was measured. The authors also investigated the toxicology of abraded particles by in-vivo instillation in rats. No difference could be observed between powders from the neat matrix material and the nanocomposites.

With the same methods, Wohlleben et al. [28] also investigated a PU/CNT nanocomposite. Measurements by SMPS show that only few particles in the nanorange are generated and a release of CNTs was not detected. Most of the released particles were bigger than $10 \mu \mathrm{m}$ and no protruding CNTs are visible on TEM images. The authors explain the missing protruding CNTs with the possibility that the elasticity of the PU allows the polymer to reflow around the CNTs during fragmentation. Cytotoxicity tests with abraded particles showed no difference between the PU and the CNT/PU samples, no acute toxicity was measured for both samples.

Methner et al. [38] investigated the release of CNFs from an epoxy composite during wet sawing, surface grinding, and belt sanding. Particles for TEM measurements were collected in the process area and at the personal breathing zone. Except a sample from belt sanding where the ventilation was turned on, all TEM samples contained free standing CNF fibers and sometimes also a larger amount of loosely agglomerated CNFs was found.

Ogura et al. [39] used a microgrinder to release particles from a $5 \mathrm{wt} \%$ of SWCNT/PP nanocomposite. The released particles were characterized by SMPS and SEM. By the usage of a thermodenuder with a working temperature of $190{ }^{\circ} \mathrm{C}$, they were able to show that more than $99.9 \%$ of the detected particles, in the range from $10 \mathrm{~nm}$ to $1000 \mathrm{~nm}$, are volatile particles that have been released by the friction heat of the grinding process. The SEM images from collected particles show particles with protruding CNTs but no free standing CNTs.

Golanski et al. [40] researched the release of CNTs from CNT/PC and CNT/PA6 composites with up to $4 \mathrm{wt} \%$ of CNTs. They simulated low abrasion with a rake mounted on a Taber Linear Abraser and high abrasion with a metallic brush. With the rake, only few particles were emitted $\left(<100 \mathrm{p} / \mathrm{cm}^{3}\right)$ and no release of CNTs was detected. With the metallic brush, two kinds of samples were investigated, one with well dispersed CNTs and one sample with poorly dispersed CNTs. No release of CNTs could be measured for the samples with well dispersed CNTs, however for the 
samples with poorly distributed CNTs, individual free standing CNTs were observed on TEM grids. The authors concluded that if there are CNT agglomerates present in the material due to a bad dispersion, these CNTs have a greater probability to be aerosolized.

Schlagenhauf et al. [27] investigated the release of CNTs from an epoxy /CNT nanocomposite with $0.1 \mathrm{wt} \%$ and $1 \mathrm{wt} \%$ CNTs. Particle generation was conducted with a Taber Abraser and all abraded particles were collected and characterized. The particle size distributions show for all samples four size modes, one in the nanorange at about $400 \mathrm{~nm}$, and three in the microrange at about $0.7 \mu \mathrm{m}, 1.2 \mu \mathrm{m}$, and $2.4 \mu \mathrm{m}$ while the sample with $1 \mathrm{wt} \%$ CNTs showed slightly larger particles for all modes. TEM sampling of released particles revealed that particles with protruding CNTs, agglomerates of CNTs, and also free standing CNTs were emitted during the abrasion process (see Figure 1). The free standing CNTs are clearly shorter than the incorporated CNTs (between $50 \mathrm{~nm}$ and $1 \mu \mathrm{m}$ ) and thus have been chopped during the abrasion process. Despite that a release of the nanofiller was observed, it was not possible to detect it with aerosol instruments. The authors explain this with the fact that both the low filler content of the CNTs in the composite and the broad size distribution of the free standing CNTs does not allow a detection by SMPS. The size mode, generated by the CNTs seemed to be within the measurement error.

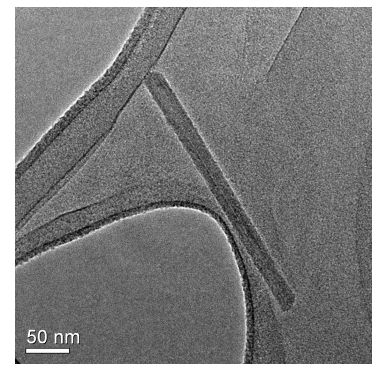

(a)

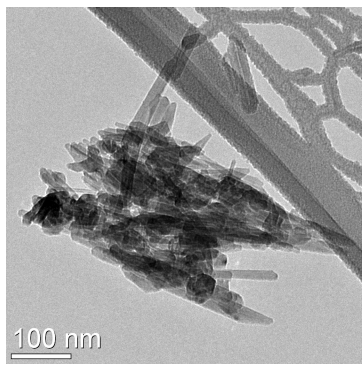

(b)

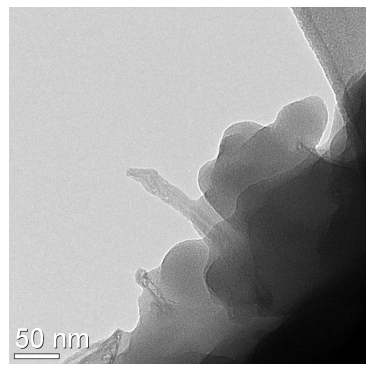

(c)

Figure 1. TEM images of abraded particles from an epoxy/CNT nanocomposite. (a) A free standing individual CNT; (b) an agglomerate of CNTs with a couple of individual CNTs scattered nearby; (c) protruding CNTs from an abraded particle [27].

Hellmann et al. [41] measured the particle release from a CNT/epoxy composite during sanding by a sanding machine. SEM measurements show particles with protruding CNTs, but no free standing CNTs have been found.

Huang et al. [42] investigated self-made and commercial available CNT/epoxy nanocomposites with up to $4 \mathrm{wt} \%$ CNTs. Abrasion was simulated with a sanding 
machine using different grit sizes and sanding speeds. The measured particle size distributions show two size modes, the first one with particles smaller than $100 \mathrm{~nm}$ contains the major amount of particles, the second mode contains particles from $500 \mathrm{~nm}$ to $5 \mu \mathrm{m}$. The measured particle concentrations increased with higher CNT loads. No free CNTs were observed except for the sample with $4 \mathrm{wt} \%$ of CNTs.

\subsection{Release Due to Weathering Processes}

Weathering can cause a weakening or even a degradation of a nanocomposite matrix and thus expose the nanofiller to the environment. It can be categorized based of three sources of weathering, UV-light, elevated temperatures, water absorption, or a combination thereof.

Degradation by UV-light causes mainly damage on the sample surface [43]. Combined with the presence of oxygen, UV-light can cause oxidation of the polymer and chain scission can form molecular products that are either volatile or lie at the surface. A common instrument to characterize the UV degradation process is the ATR-FTIR [44]. It allows to follow different degradation products and to define a polymer specific degradation index $[43,45,46]$.

Elevated temperatures can cause thermooxidation with similar degradation products as UV degradation [44]. Thermooxidation first affects the surface layer, this is important for coatings and adhesives. Depending on the oxygen diffusion kinetics, also bulk properties of composites will be affected [47].

Degradation by water uptake can influence the bulk properties of polymers and their composites. The affected properties among others are the stiffness, the interfacial strength, the damping ratio, weakening of the fiber-matrix interface in composites, plasticization, swelling, and softening [48]. The maximum water uptake depends on the relative humidity but not on the temperature [49]. Further e.g., for epoxy, different species of water are observed in the polymer, it can be free or bound to the matrix [50]. Different models for the water uptake of nanocomposites have been developed and tested by Liu et al. [51,52].

The reviewed papers of this section are summarized in Table 3.

Nguyen et al. and Petersen et al. [53-55] investigated the degradation of a CNT/epoxy nanocomposite under intensive UV-light [63]. They measured the weight loss of the samples and the degradation of the matrix by ATR-FTIR. The results show that the composite degrades slower than the neat epoxy. In contrary to a $\mathrm{SiO}_{2}$ / epoxy that the same research group investigated [46], a release of the nanofiller could not be measured. Due to degradation of the matrix material, CNTs formed a network on the surface of the samples but an analysis of released particles did not show free standing CNTs. Further, the scratch resistance of the CNT layer was tested. It could be shown that CNT network on the weathered epoxy surface is more mechanically resistant to scratching than the neat epoxy. The authors conclude 
that this finding indicates that it is unlikely that the CNTs are readily released into the environment.

The degradation by UV-light of a CNT/PP and a CNT/PE composite has been investigated by Bocchini et al. [56]. The degradation of the matrix was measured by ATR-FTIR and the results show that the two matrix materials behave differently. The CNT/PP composite shows a similar behavior as the analyzed CNT/epoxy nanocomposite of Nguyen et al. [53,54], the CNTs are adsorbing the UV-light and thus, less degradation compared to the neat PP can be measured. For the $\mathrm{CNT} / \mathrm{PE}$ composite, almost no difference between polymer and composite can be measured. The authors propose that besides the photooxidation by the UV light, also thermooxdiation plays a role during the degradation process. Since the CNTs are converting light into heat, they conclude that the composites have a higher temperature during the degradation experiment and thus have a higher thermooxidation. For the PE composite, the positive effect of the CNTs seems to be balanced by the negative effects of the higher temperature.

Besides the abrasion processes, Wohlleben et al. [25,28] and Hirth et al. [57] investigated the UV-degradation of CNT/POM, CNT/epoxy and CNT/PU nanocomposites. During the experiment, the samples were protected by covers to ensure that no loose particles are blown or washed away. Afterwards, either the loose particles on the sample surface, or particles that have been released into water during sonication have been analyzed by TEM. For the CNT/epoxy composite, the TEM images show particles with embedded or protruding CNTs. By application of high shear forces, also free standing CNTs were observed.

Asmatulu et al. [58] investigated the resistance against weathering of neat epoxy and CNT/epoxy coatings. The coatings were exposed to UV-light only or to a combination of UV-light and salt fog (according to ASTM B117). The coatings with incorporated CNTs showed a slower weight loss and smaller cracks were generated on the surface compared to the neat epoxy.

Orlov et al. [59] degraded different nanocomposites with CNTs and amino functionalized CNTs with a combination of UV light and moisture. The authors observed degradation of the matrix by FTIR and the formation of cracks by microscope. SEM imaging of samples revealed that loose CNTs can be found on the samples surfaces as well as within the cracks. Even though the amino functionalized CNTs were better dispersed, the polymer matrix was much less homogeneous. This might be explained with a lower degree of polymer cross linking compared to the composite with neat CNTs.

Vilar et al. [60] investigated a CNT/PA6 composite. They weathered the samples under UV light in combination with moisture. After the weathering process, SEM images showed CNTs that are exposed to the environment on the sample surface. The authors also calcinated the samples in order to regain the nanofillers. In contrary 
to the unexposed samples, the calcination of aged samples caused the release of free standing CNTs.

Table 3. CNT release studies—Weathering.

\begin{tabular}{|c|c|c|c|}
\hline Study & Material & Weathering Process & Nanofiller Release? \\
\hline $\begin{array}{l}\text { Nguyen et al. [53,54] } \\
\text { and Petersen et al. [55] }\end{array}$ & CNT/epoxy composite & UV degradation & $\begin{array}{l}\text { Formation of a CNT layer on } \\
\text { the surface, no release of CNTs }\end{array}$ \\
\hline Bocchini et al. [56] & $\begin{array}{l}\mathrm{CNT} / \mathrm{PP} \text { and } \\
\mathrm{CNT} / \mathrm{PE} \text { composites }\end{array}$ & UV degradation & Not measured \\
\hline $\begin{array}{l}\text { Wohlleben et al. }[25,28] \\
\text { and Hirth et al. [57] }\end{array}$ & $\begin{array}{l}\text { CNT/POM, CNT/epoxy, } \\
\text { and CNT/PU composites }\end{array}$ & $\begin{array}{l}\text { UV degradation with } \\
\text { or without moisture }\end{array}$ & $\begin{array}{l}\text { Release of CNTs only when } \\
\text { high shear forces are applied on } \\
\text { released particles }\end{array}$ \\
\hline Asmatulu et al. [58] & CNT/epoxy composite & $\begin{array}{l}\text { UV degradation } \\
\text { only or with salt fog }\end{array}$ & Not measured \\
\hline Orlov et al. [59] & $\begin{array}{l}\text { Different CNT/polymer } \\
\text { composites }\end{array}$ & $\begin{array}{l}\text { UV degradation } \\
\text { with moisture }\end{array}$ & $\begin{array}{l}\text { Loose CNTs on surface and in } \\
\text { cracks are observed by SEM, a } \\
\text { release of CNTs was not measured }\end{array}$ \\
\hline Vilar et al. [60] & CNT/PA6 composite & $\begin{array}{l}\text { UV degradation } \\
\text { with moisture }\end{array}$ & $\begin{array}{l}\text { Exposed CNTs on the sample } \\
\text { surface, weathered and calcinated } \\
\text { samples showed a release of CNTs }\end{array}$ \\
\hline Busquets-Fitè et al. [61] & $\begin{array}{l}\mathrm{CNT} / \mathrm{PP}, \mathrm{CNT} / \mathrm{EVA} \text {, and } \\
\mathrm{CNT} / \mathrm{PA} 6 \text { composites }\end{array}$ & $\begin{array}{l}\text { UV degradation } \\
\text { with moisture }\end{array}$ & No release of CNTs \\
\hline Ging et al. [62] & $\begin{array}{l}\text { CNT/epoxy composite } \\
\text { with neat and amino } \\
\text { functionalized CNTs }\end{array}$ & $\begin{array}{l}\text { UV degradation } \\
\text { with high humidity }\end{array}$ & $\begin{array}{l}\text { Formation of a CNT layer on the } \\
\text { surface, release not measured }\end{array}$ \\
\hline Barkoula et al. [48] & $\begin{array}{l}\text { CNT/CRP/epoxy } \\
\text { composite }\end{array}$ & Water bath & Not measured \\
\hline Starkova et al. [49] & CNT/epoxy composite & Humidity & Not measured \\
\hline
\end{tabular}

Busquets-Fitè et al. [61] weathered CNT/PP, CNT/EVA, and CNT/PA6 nanocomposites under UV light combined with moisture. They gathered the aging water and collected the released particles. No released CNTs have been found.

The degradation of a CNT/epoxy nanocomposite with neat and amino functionalized CNTs by UV light and elevated humidity has been investigated by Ging et al. [62]. Images by SEM revealed that several forms of CNTs can be found on the weathered surface, free standing agglomerates of CNTs, partially exposed CNTs due to crack formation, and encapsulated CNTs (see Figure 2). The authors conclude that there is a possibility that CNTs can be released into the environment after the matrix is degraded, but it has to be considered that this process takes time and thus the releasing rate would be rather low. Further, the authors milled the 
exposed nanocomposite films and tested the toxicity of the particles with Drosophila larvae. In contrary to the neat CNTs, the embedded CNTs were not toxic.

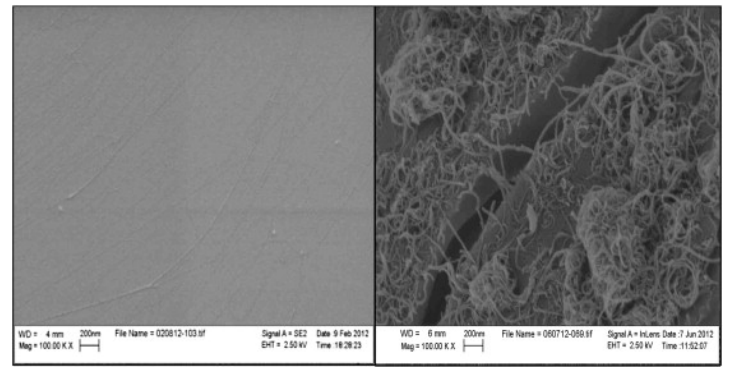

Figure 2. Impact of UV light on a CNT/epoxy nanocomposite: the initial flat surface (left) gets cracks and unprotected CNTs are forming a layer (right). Reprinted from Science of the Total Environment, 473-474, J. Ging et al., Development of a conceptual framework for evaluation of nanomaterials release from nanocomposites: Environmental and toxicological implications, p. 9-19, Copyright (C) 2014, with permission from Elsevier [62].

Changes in the electrical conductivity of a CNT nanocomposite can be used to monitor its degradation. Therefore Barkoula et al. [48] made an attempt to correlate the water uptake of a CNT/CRP/epoxy composite with the change of the electrical resistance. They also compared the water uptake of a CNT/epoxy composite with the neat epoxy. The neat epoxy system exhibited a slightly lower water uptake than the modified systems. These results are in good agreement with the results of Starkova et al. [49] where the water uptake of a CNT/epoxy composite was measured under different relative humidities and different temperatures. At lower temperatures, it could be shown that the water uptake rate for the neat epoxy is faster in comparison with the nanocomposites but the equilibrium weight gain was not influenced by the nanofiller. Further, the addition of CNTs to the epoxy also did not influence the swelling of the samples.

\subsection{Release Due to Fire}

Due to improper disposal by fire or by accidental burning of CNT nanocomposites, the nanofiller can be released into the environment since the decomposition temperature of CNTs is much higher than of the polymer matrix, they can be stable up to $600^{\circ} \mathrm{C}$ [64]. So far, no detailed study has been published that investigates the release of CNTs from nanocomposites due to an event of fire. However, in the field of fire inhibition, CNT/polymer nanocomposites have been already investigated and the analysis of the produced char can give an indication of the possible release of CNTs into the environment. 
The reviewed papers of this section are summarized in Table 4 .

Table 4. CNT release studies-Fire.

\begin{tabular}{|c|c|c|}
\hline Study & Material & Results \\
\hline Kashiwagi et al. [7,65-67] & $\begin{array}{l}\mathrm{CNT} / \mathrm{PP}, \mathrm{CNT} / \mathrm{PS} \text {, and } \\
\mathrm{CNT} / \mathrm{PMMA} \text { composites }\end{array}$ & $\begin{array}{l}\text { Partially oxidized CNTs found in char with } \\
\text { oxidized CNT catalyst }\end{array}$ \\
\hline Schartel et al. [68] & CNT/PA6 composite & Residuals consisted of a CNT network \\
\hline Verdejo et al. [69] & $\mathrm{CNT} /$ silicone foam & Residuals consisted of a CNT network \\
\hline Kim et al. [70] & CNT/PEN composite & Residuals consisted of a CNT network \\
\hline Fu et al. [71] & $\begin{array}{l}\mathrm{CNT} / \text { wood/PE and } \\
\mathrm{CNT}-\mathrm{OH} / \text { wood/PE composites }\end{array}$ & $\begin{array}{l}\text { Free CNTs on char surface for } \mathrm{CNT} / \text { wood/PE } \\
\text { composite, no CNTs found on char for } \mathrm{CNT}-\mathrm{OH} / \\
\text { wood/PE composite }\end{array}$ \\
\hline $\begin{array}{l}\text { Zammarano et al. [72], } \\
\text { Nyden et al. [73], } \\
\text { Uddin et al. }[74]\end{array}$ & CNF/PU foam & $\begin{array}{l}\text { No CNFs in smoke, free CNFs found in char } \\
\text { aerosolization of CNFs from the char due to shaking }\end{array}$ \\
\hline Dittrich et al. [75] & $\begin{array}{l}\text { Different carbonaceous } \\
\text { nanofillers in PP composites }\end{array}$ & $\begin{array}{l}\text { Formation of residual protection layer, no further } \\
\text { investigation of the char }\end{array}$ \\
\hline Bouillard et al. [76] & CNT/ABS composite & $\begin{array}{l}\text { Release of free CNTs and agglomerates of CNTs } \\
\text { into air during burning in furnace }\end{array}$ \\
\hline
\end{tabular}

Kashiwagi et al. [7] studied the mechanism of fire inhibition by CNTs with a PP nanocomposite. The experiments showed that the present CNTs modified the thermal and oxidation properties of the PP matrix. Cone calorimetry measurements showed that the heat release of the PP nanocomposite is significantly reduced compared to the neat PP. The analysis of the sample residues revealed that partially oxidized agglomerated CNTs are present in the char (see Figure 3). Further, the color of the char was red because the iron catalyst in the nanotubes was oxidized. The authors concluded that the formed CNT network increased the mechanical integrity of a protective layer which could act as a thermal insulation layer and also as a barrier for evolved degradation products to the gas phase.

Further experiments by the same group with PP, PS, and PMMA nanocomposites confirmed these results [65-67]. For all samples, a protection layer made of CNTs was formed during cone calorimeter experiments. The network layer showed physical integrity and had about the same mass as the initial mass of the CNTs in the nanocomposite.

Schartel et al. [68] investigated the fire resistance of a CNT/PA6 nanocomposite. They showed that the CNT network remained in the composite, influenced the melt viscosity, and thus prevented dripping and flowing of the burning sample. The authors concluded that CNTs may be a highly interesting filling material for flame retardation, but only for distinct systems and scenarios. Since dripping is inhibited by 
the CNT network, the conversion of the material is increased, this might be negative for passing flammability tests.

Verdejo et al. [69] used CNTs to change the fire properties of silicone foams. Also for this study, a CNT network was observed in the residues of the burned foams.

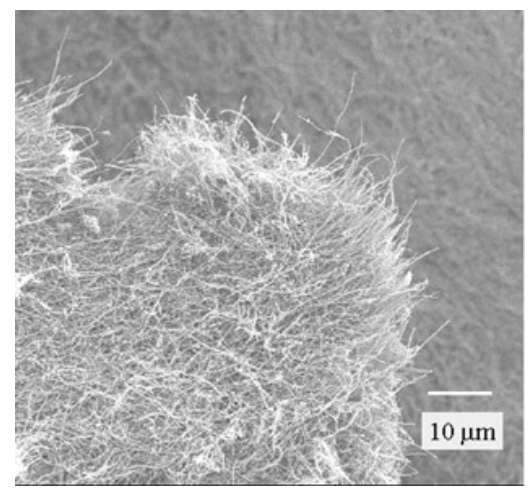

Figure 3. SEM picture of the residues from a burned CNT/PP nanocomposite. Reprinted from Macromol. Rapid Commun., 23, T. Kashiwagi et al., Thermal Degradation and Flammability Properties of Poly(propylene)/Carbon Nanotube Composites, p. 761-765, Copyright (c) 2002, with permission from Wiley-VCH [7].

Kim et al. [70] investigated a CNT/PEN nanocomposite. The thermal stability could be enhanced by adding CNTs to PEN, but the thermal decomposition kinetics depended strongly on the CNT content. SEM analysis of the residues showed also for this nanocomposite the formation of a CNT network during the decomposition process.

Fu et al. [71] created a CNT/wood/PE nanocomposite that contained either neat CNTs or hydroxylated CNTs (CNT-OH). The hydroxylated CNTs showed a slightly better flame retardancy due to their improved interfacial compatibilization between CNT-OH and wood flour as well as the polymer matrix. SEM images of the char residues of the composite with untreated CNTs showed free CNTs on the char surface. For the composite with functionalized CNTs, no free CNTs could be detected. Zammarano et al. [72] produced a PU foam with incorporated CNFs und tested the flammability of the composite by a cone calorimeter. Due to the incorporated CNFs, flame spread was reduced by preventing the heat transfer of burning material to adjacent surfaces. A thermal stable entangled fiber network was formed that also prevented the collapse of the foam. Smoke and char of this burned CNF/PU foam was then analyzed by Nyden et al. [73] and Uddin et al. [74]. They burned the composite and collected samples from the released smoke on filters. Further, they also suspended the char in water in order to collect particles for electron microscopy analysis. The analysis of the samples showed that free standing CNFs are present in 
the char but they were not airborne. The authors conclude that any airborne CNF has been destroyed in the flames. Further, collected char was shaked to see whether CNFs can be released into air. The measurements showed that the particle concentration was increased by an order of magnitude. The released particles further were trapped in water and by measuring their concentration, the rate of aerosolization of the CNFs was approximately determined to $1.4 \mathrm{mg} /$ hour.

Dittrich et al. [75] compared the flame retardance properties of different carbonaceous nanofillers. All filler materials enhanced the flame retardancy of the PP matrix by the formation of a protection layer. Functionalized graphene showed the highest potential as flame retardant material.

Bouillard et al. [76] was the first study to investigate the formation of airborne CNTs during the combustion of a CNT nanocomposite. They used a CNT/ABS composite with $3 \mathrm{wt} \%$ of CNTs, combusted the sample in a furnace and collected released particles on TEM grids. Analysis by TEM revealed that free standing CNTs and also agglomerates of CNTs were released into the air. The authors report that the numbers were quite significant posing a possible sanitary risk in the case of accidental scenarios.

\section{Conclusions}

A summary for all investigated release studies is given in Table 5, it shows that there are still some gaps that have to be filled. Further, the CNT release from other polymers that have been considered as matrix material for CNT nanocomposites have not been investigated at all, e.g., polyvinyl acetate (PVA) [77], polyimide (PI) [78], polytrimethylene terephthalate (PTT) [79], or vinylester/polyester [80].

The studies that investigated the release due to mechanical impact do not give a coherent picture. It stands out that both studies that investigated the release of CNFs from composites find airborne CNFs in the breathing area, not only under dry conditions but also for wet sawing. The number of studies however is limited and no universal conclusions can be drawn.

For CNT nanocomposites, it can be concluded that the expected release scenarios include free standing CNTs, agglomerated CNTs, and particles with- and without protruding CNTs. Depending on the applied shear force during the abrasion process, it is also expected that released CNTs will be shorter than the processed CNTs in the composite. Normally during abrasion processes, the particle concentration in air is too low for particle agglomeration. This means that the finding of agglomerates can indicate a poor distribution of the CNTs in the investigated nanocomposite. 
Table 5. Release studies for all investigated polymers.

\begin{tabular}{cccc}
\hline Polymer & Abrasion & Weathering & Fire \\
\hline ABS & & & {$[76]$} \\
Epoxy & {$[27,34-38,41,42]$} & {$[48,49,53-55,57,58,62]$} & \\
EVA & & {$[61]$} & \\
PA6 & {$[40]$} & {$[60,61]$} & {$[68]$} \\
PC & {$[40]$} & & \\
PE & & {$[56]$} & {$[71]$} \\
PEN & & & {$[70]$} \\
PMMA & & & {$[66,67]$} \\
POM & {$[25]$} & {$[25]$} & {$[7,65,75]$} \\
PP & {$[39]$} & {$[56,61]$} & {$[67]$} \\
PS & & & {$[72-74]$} \\
PU & {$[28]$} & {$[28]$} & {$[69]$} \\
Silicone & & &
\end{tabular}

As already discussed by Hirth et al. [57], the release abilities of CNT/polymer composites can be divided into two categories. The first category includes the ductile matrix materials. A release of CNTs has not been observed for those materials. It seems that the ductile matrix is able to reflow around the CNTs during fragmentation [28], for those composites, no or only few protruding CNTs from abraded particles are expected. The second category includes brittle materials, here protruding CNTs from abraded particles are observed for all tested composites. For this category, also a release of CNTs has been observed but only for few tested materials or under special circumstances as a high filler loading [42] or the presence of agglomerated CNTs in the material [40]. If other factors, such as the influence of the filler-matrix interface, play a role in the CNT release abilities of brittle composites still has to be evaluated. An approach to reduce or even to prevent the release of CNTs due to abrasion can be the functionalization of CNTs. E.g., for epoxy composites, functionalized CNTs can be better dispersed in the epoxy resin [81] and the interaction between CNT and matrix can be improved [82].

For the weathering studies, no differences among the investigated $\mathrm{CNT}$ / polymer composites can be found. All of them expose CNTs to the environment when the matrix is degraded by UV-light. The CNTs form a network and are not easily to detach from the samples. Even though already several studies have been published with confirming results, it is not possible to draw a conclusion for a risk assessment of the considered scenarios since only the worst case has been investigated where the composite has not been protected by additives. Further, the investigation of a CNT release due to a combination of weathering and abrasion processes would 
be interesting since it is closer to the real life (e.g., usage of outdoor materials or polishing of a dulled surface). Also toxicity studies on this subject are desired. The formed CNT layer on the surface of weathered composites could be a source of a high quantity of released free standing CNTs and thus maybe pose a health risk. For the weathering by elevated temperatures and water, no conclusions can be drawn since their effect on the CNT release has not been investigated yet.

In contrary to incineration where under high temperatures CNTs are destroyed [83], a fire does not degrade all CNTs in composites, they even can be used as flame retardants. All the fire release studies show that during a fire incident, a network of CNTs is formed in the char. It hinders a dripping of molten composite and influences the degradation properties. For future studies, despite the positive flame retardant properties, also exposure to CNTs has to be regarded. For CNFs, it has been already shown that they can be released from the char into the air. For CNTs, such measurements are still missing. Further, the toxicity of the inhalable fraction of the released particles would be important. As a fire is a single incident, only short term exposure would have to be considered.

Acknowledgments: This study was financed by the Swiss National Science Foundation (NFP 64), "Evaluation platform for safety and environment risks of carbon nanotube reinforced nanocomposites", 406440_131286.

Conflicts of Interest: The authors declare no conflicts of interest.

\section{References}

1. Coleman, J.N.; Khan, U.; Gun'ko, Y.K. Mechanical reinforcement of polymers using carbon nanotubes. Adv. Mater. 2006, 18, 689-706.

2. Coleman, J.N.; Khan, U.; Blau, W.J.; Gun'ko, Y.K. Small but strong: A review of the mechanical properties of carbon nanotube-polymer composites. Carbon 2006, 44, 1624-1652.

3. Sun, L.Y.; Gibson, R.F.; Gordaninejad, F.; Suhr, J. Energy absorption capability of nanocomposites: A review. Composit. Sci. Technol. 2009, 69, 2392-2409.

4. Giraldo, L.F.; Brostow, W.; Devaux, E.; Lopez, B.L.; Perez, L.D. Scratch and wear resistance of polyamide 6 reinforced with multiwall carbon nanotubes. J. Nanosci. Nanotechnol. 2008, 8, 1-8.

5. Huang, H.; Liu, C.T.H.; Wu, Y.; Fan, S. Aligned carbon nanotube composite films for thermal management. Adv. Mater. 2005, 17, 1652-1656.

6. Hollertz, R.; Chatterjee, S.; Gutmann, H.; Geiger, T.; Nueesch, F.A.; Chu, B.T.T. Improvement of toughness and electrical properties of epoxy composites with carbon nanotubes prepared by industrially relevant processes. Nanotechnology 2011, 22, doi:10.1088/0957-4484/22/12/125702.

7. Kashiwagi, T.; Grulke, E.; Hilding, J.; Harris, R.; Awad, W.; Douglas, J. Thermal degradation and flammability properties of poly(propylene)/carbon nanotube composites. Macromol. Rapid Commun. 2002, 23, 761-765. 
8. Breuer, O.; Sundararaj, U. Big returns from small fibers: A review of polymer/carbon nanotube composites. Polym. Composit. 2004, 25, 630-645.

9. Kingston, C.; Zepp, R.; Andrady, A.; Boverhof, D.; Fehir, R.; Hawkins, D.; Roberts, J.; Sayre, P.; Shelton, B.; Sultan, Y.; Vejins, V.; Wohlleben, W. Release Characteristics of Selected Carbon Nanotube Polymer Composites. Carbon 2014, 68, 33-57.

10. Muller, L.; Riediker, M.; Wick, P.; Mohr, M.; Gehr, P.; Rothen-Rutishauser, B. Oxidative stress and inflammation response after nanoparticle exposure: Differences between human lung cell monocultures and an advanced three-dimensional model of the human epithelial airways. J. R. Soc. Interface 2010, 7, S27-S40.

11. Xia, T.; Li, N.; Nel, A.E. Potential Health Impact of Nanoparticles. Annu. Rev. Public Health 2009, 30, 137-150.

12. Helland, A.; Wick, P.; Koehler, A.; Schmid, K.; Som, C. Reviewing the environmental and human health knowledge base of carbon nanotubes. Environ. Health Perspect. 2007, $115,1125-1131$.

13. Pulskamp, K.; Diabate, S.; Krug, H.F. Carbon nanotubes show no sign of acute toxicity but induce intracellular reactive oxygen species in dependence on contaminants. Toxicol. Lett. 2007, 168, 58-74.

14. Ma-Hock, L.; Treumann, S.; Strauss, V.; Brill, S.; Luizi, F.; Mertler, M.; Wiench, K.; Gamer, A.O.; van Ravenzwaay, B.; Landsiedel, R. Inhalation Toxicity of Multiwall Carbon Nanotubes in Rats Exposed for 3 Months. Toxicol. Sci. 2009, 112, 468-481.

15. Palomaki, J.; Valimaki, E.; Sund, J.; Vippola, M.; Clausen, P.A.; Jensen, K.A.; Savolainen, K.; Matikainen, S.; Alenius, H. Long, Needle-like Carbon Nanotubes and Asbestos Activate the NLRP3 Inflammasome through a Similar Mechanism. ACS Nano 2011, 5, 6861-6870.

16. Thurnherr, T.; Brandenberger, C.; Fischer, K.; Diener, L.; Manser, P.; Maeder-Althaus, X.; Kaiser, J.P.; Krug, H.F.; Rothen-Rutishauser, B.; Wick, P. A comparison of acute and long-term effects of industrial multiwalled carbon nanotubes on human lung and immune cells in vitro. Toxicol. Lett. 2011, 200, 176-186.

17. Koehler, A.R.; Som, C.; Helland, A.; Gottschalk, F. Studying the potential release of carbon nanotubes throughout the application life cycle. J. Clean. Prod. 2008, 16, 927-937.

18. Wardak, A.; Gorman, M.E.; Swami, N.; Deshpande, S. Identification of risks in the life cycle of nanotechnology-based products. J. Ind. Ecol. 2008, 12, 435-448.

19. Abbott, L.C.; Maynard, A.D. Exposure Assessment Approaches for Engineered Nanomaterials. Risk Anal. 2010, 30, 1634-1644.

20. Gottschalk, F.; Nowack, B. The release of engineered nanomaterials to the environment. J. Environ. Monit. 2011, 13, 1145-1155.

21. Petersen, E.J.; Zhang, L.W.; Mattison, N.T.; O'Carroll, D.M.; Whelton, A.J.; Uddin, N.; Nguyen, T.; Huang, Q.G.; Henry, T.B.; Holbrook, R.D.; et al. Potential Release Pathways, Environmental Fate, And Ecological Risks of Carbon Nanotubes. Environ. Sci. Technol. 2011, 45, 9837-9856. 
22. Nowack, B.; Ranville, J.F.; Diamond, S.; Gallego-Urrea, J.A.; Metcalfe, C.; Rose, J.; Horne, N.; Koelmans, A.A.; Klaine, S.J. Potential scenarios for nanomaterial release and subsequent alteration in the environment. Environ. Toxicol. Chem. 2012, 31, 50-59.

23. Fissan, H.; Horn, H.G.; Stahlmecke, B.; Wang, J. From nanoobject release of (Bio)nanomaterials to exposure. BioNanoMaterials 2013, 14, 37-47.

24. Vorbau, M.; Hillemann, L.; Stintz, M. Method for the characterization of the abrasion induced nanoparticle release into air from surface coatings. J. Aerosol Sci. 2009, 40, 209-217.

25. Wohlleben, W.; Brill, S.; Meier, M.W.; Mertler, M.; Cox, G.; Hirth, S.; von Vacano, B.; Strauss, V.; Treumann, S.; Wiench, K.; et al. On the Lifecycle of Nanocomposites: Comparing Released Fragments and their In-Vivo Hazards from Three Release Mechanisms and Four Nanocomposites. Small 2011, 7, 2384-2395.

26. Golanski, L.; Gaborieau, A.; Guiot, A.; Uzu, G.; Chatenet, J.; Tardif, F. Characterization of abrasion-induced nanoparticle release from paints into liquids and air. J. Phys. Conf. Series 2011, 304, 012062.

27. Schlagenhauf, L.; Chu, B.T.T.; Buha, J.; Nueesch, F.; Wang, J. Release of Carbon Nanotubes from an Epoxy-Based Nanocomposite during an Abrasion Process. Environ. Sci. Technol. 2012, 46, 7366-7372.

28. Wohlleben, W.; Meier, M.W.; Vogel, S.; Landsiedel, R.; Cox, G.; Hirth, S.; Tomovic, Z. Elastic CNT-polyurethane nanocomposite: synthesis, performance and assessment of fragments released during use. Nanoscale 2013, 5, 369-380.

29. Koponen, I.K.; Jensen, K.A.; Schneider, T. Sanding dust from nanoparticlecontaining paints: Physical characterisation. J. Phys. Conf. Series 2009, 151, doi:10.1088/1742-6596/151/1/012048.

30. Koponen, I.K.; Jensen, K.A.; Schneider, T. Comparison of dust released from sanding conventional and nanoparticle-doped wall and wood coatings. J. Expo. Sci. Environ. Epidemiol. 2011, 21, 408-418.

31. Gohler, D.; Stintz, M.; Hillemann, L.; Vorbau, M. Characterization of Nanoparticle Release from Surface Coatings by the Simulation of a Sanding Process. Ann. Occup. Hyg. 2010, $54,615-624$.

32. Kuhlbusch, T.A.J.; Asbach, C.; Fissan, H.; Goehler, D.; Stintz, M. Nanoparticle exposure at nanotechnology workplaces: A review. Part. Fibre Toxicol. 2011, 8, doi:10.1186/1743-8977-8-22.

33. Mazzuckelli, L.F.; Methner, M.M.; Birch, M.E.; Evans, D.E.; Ku, B.K.; Crouch, K.; Hoover, M.D. Identification and characterization of potential sources of worker exposure to carbon nanofibers during polymer composite laboratory operations. J. Occup. Environ. Hyg. 2007, 4, D125-D130.

34. Bello, D.; Wardle, B.L.; Yamamoto, N.; deVilloria, R.G.; Garcia, E.J.; Hart, A.J.; Ahn, K.; Ellenbecker, M.J.; Hallock, M. Exposure to nanoscale particles and fibers during machining of hybrid advanced composites containing carbon nanotubes. J. Nanoparticle Res. 2009, 11, 231-249. 
35. Bello, D.; Wardle, B.L.; Zhang, J.; Yamamoto, N.; Santeufemio, C.; Hallock, M.; Virji, M.A. Characterization of Exposures to Nanoscale Particles and Fibers During Solid Core Drilling of Hybrid Carbon Nanotube Advanced Composites. Int. J. Occup. Environ. Health 2010, 16, 434-450.

36. Bello, D.; Wardle, B.L.; Yamamoto, N.; deVilloria, R.G.; Hallock, M. Exposures to Nanoscale Particles and Fibers During Handling,Processing, and Machining of Nanocomposites and Nanoengineered Composites Reinforced with Aligned Carbon Nanotubes. Proceedings of the 17th International conference on composite materials (ICCM), Edinburgh, Scotland, July 27-31, 2011.

37. Cena, L.G.; Peters, T.M. Characterization and Control of Airborne Particles Emitted During Production of Epoxy/Carbon Nanotube Nanocomposites. J. Occup. Environ. Hyg. 2011, 8, 86-92.

38. Methner, M.; Crawford, C.; Geraci, C. Evaluation of the Potential Airborne Release of Carbon Nanofibers During the Preparation, Grinding, and Cutting of Epoxy-Based Nanocomposite Material. J. Occup. Environ. Hyg. 2012, 9, 308-318.

39. Ogura, I.; Kotake, M.; Shigeta, M.; Uejima, M.; Saito, K.; Hashimoto, N.; Kishimoto, A. Potential release of carbon nanotubes from their composites during grinding. J. Phys. Conf. Series 2013, 429, doi:10.1088/1742-6596/429/1/012049.

40. Golanski, L.; Guiot, A.; Pras, M.; Malarde, M.; Tardif, F. Release-ability of nano fillers from different nanomaterials (toward the acceptability of nanoproduct). J. Nanoparticle Res. 2012, 14, doi:10.1007/s11051-012-0962-x.

41. Hellmann, A.; Schmidt, K.; Ripperger, S.; Berges, M. Release of ultrafine dusts during the machining of nanocomposites. Gefahrst. Reinhalt. Luft 2012, 72, 473-476.

42. Huang, G.; Park, J.; Cena, L.; Shelton, B.; Peters, T. Evaluation of airborne particle emissions from commercial products containing carbon nanotubes. J. Nanoparticle Res. 2012, 14, 1231.

43. Mailhot, B.; Morlat-Thérias, S.; Bussière, P.O.; Gardette, J.L. Study of the Degradation of an Epoxy/Amine Resin, 2. Macromol. Chem. Phys. 2005, 206, 585-591.

44. Mailhot, N.; Morlat-Theias, S.; Ouahioune, M.; Gardette, J.L. Study of the degradation of an epoxy/amine resin, 1 photo- and thermo-chemical mechanisms. Macromol. Chem. Phys. 2005, 206, 575-584.

45. Zhang, Y.; Maxted, J.; Barber, A.; Lowe, C.; Smith, R. The durability of clear polyurethane coil coatings studied by FTIR peak fitting. Polym. Degrad. Stab. 2013, 98, 527-534.

46. Nguyen, T.; Pellegrin, B.; Bernard, C.; Rabb, S.; Stuztman, P.; Gorham, J.M.; Gu, X.; Yu, L.L.; Chin, J.W. Characterization of Surface Accumulation and Release of Nanosilica During Irradiation of Polymer Nanocomposites by Ultraviolet Light. J. Nanosci. Nanotechnol. 2012, 12, 6202-6215.

47. Celina, M.C.; Dayile, A.R.; Quintana, A. A perspective on the inherent oxidation sensitivity of epoxy materials. Polymer 2013, 54, 3290-3296.

48. Barkoula, N.M.; Paipetis, A.; Matikas, T.; Vavouliotis, A.; Karapappas, P.; Kostopoulos, V. Environmental degradation of carbon nanotube-modified composite laminates: A study of electrical resistivity. Mech. Composit. Mater. 2009, 45, 21-32. 
49. Starkova, O.; Buschhorn, S.T.; Mannov, E.; Schulte, K.; Aniskevich, A. Water transport in epoxy/MWCNT composites. Eur. Polym. J. 2013, 49, 2138-2148.

50. Popineau, S.; Rondeau-Mouro, C.; Sulpice-Gaillet, C.; Shanahan, M.E.R. Free/bound water absorption in an epoxy adhesive. Polymer 2005, 46, 10733-10740.

51. Liu, W.; Hoa, S.V.; Pugh, M. Water uptake of epoxy-clay nanocomposites: Model development. Composit. Sci. Technol. 2007, 67, 3308-3315.

52. Liu, W.; Hoa, S.V.; Pugh, M. Water uptake of epoxy-clay nanocomposites: Experiments and model validation. Composit. Sci. Technol. 2008, 68, 2066-2072.

53. Nguyen, T.; Pellegrin, B.; Mermet, L.; Shapiro, A.; Gu, X.; Chin, J. Network aggregation of CNTs at the surface of epoxy/MWCNT composite exposed to UV radiation. In Proceedings of the Nanotechnology 2009: Fabrication, Particles, Characterization, MEMS, Electronics and Photonics-Technical Proceedings of the 2009 NSTI Nanotechnology Conference and Expo, NSTI-Nanotech, Houston, TX, May 3-7 2009; Volume 1, pp. 90-93.

54. Nguyen, T.; Pellegrin, B.; Bernard, C.; Gu, X.; Gorham, J.M.; Stutzman, P.; Stanley, D.; Shapiro, A.; Byrd, E.; Hettenhouser, R.; Chin, J. Fate of nanoparticles during life cycle of polymer nanocomposites. J. Phys. Conf. Series 2011, 304, 012060.

55. Petersen, E.J.; Lam, T.; Gorham, J.M.; Scott, K.C.; Long, C.J.; Stanley, D.; Sharma, R.; Alexander Liddle, J.; Pellegrin, B.; Nguyen, T. Methods to assess the impact of UV irradiation on the surface chemistry and structure of multiwall carbon nanotube epoxy nanocomposites. Carbon 2014, 69, 194-205.

56. Bocchini, S.; Di Blasio, A.; Frache, A. Influence of MWNT on Polypropylene and Polyethylene Photooxidation. Macromol. Symp. 2011, 301, 16-22.

57. Hirth, S.; Cena, L.; Cox, G.; Tomović, Z.; Peters, T.; Wohlleben, W. Scenarios and methods that induce protruding or released CNTs after degradation of nanocomposite materials Technology Transfer and Commercialization of Nanotechnology. J. Nanoparticle Res. 2013, 15, doi:10.1007/s11051-013-1504-x.

58. Asmatulu, R.; Mahmud, G.A.; Hille, C.; Misak, H.E. Effects of UV degradation on surface hydrophobicity, crack, and thickness of MWCNT-based nanocomposite coatings. Prog. Org. Coat. 2011, 72, 553-561.

59. Orlov, A.; Ramakrishnan, G.; Ging, J.; Hubert, A.; Feka, P.; Korach, C.S. Evaluating safety and stability of CNT nanocomposites exposed to environmental conditions. In Proceedings of the Technical Proceedings of the 2012 NSTI Nanotechnology Conference and Expo, Santa Clara, CA, June 18-21, 2012; pp. 335-337.

60. Vilar, G.; Fernández-Rosas, E.; Puntes, V.; Jamier, V.; Aubouy, L.; Vázquez-Campos, S. Monitoring migration and transformation of nanomaterials in polymeric composites during accelerated aging. J. Phys. Conf. Series 2013, 429, doi:10.1088/1742-6596/429/1/012044.

61. Busquets-Fité, M.; Fernandez, E.; Janer, G.; Vilar, G.; Vázquez-Campos, S.; Zanasca, R.; Citterio, C.; Mercante, L.; Puntes, V. Exploring release and recovery of nanomaterials from commercial polymeric nanocomposites. J. Phys. Conf. Series 2013, 429, doi:10.1088/ 1742-6596/429/1/012048. 
62. Ging, J.; Tejerina-Anton, R.; Ramakrishnan, G.; Nielsen, M.; Murphy, K.; Gorham, J.M.; Nguyen, T.; Orlov, A. Development of a conceptual framework for evaluation of nanomaterials release from nanocomposites: Environmental and toxicological implications. Sci. Total Environ. 2014, 473-474, 9-19.

63. Chin, J.; Byrd, E.; Embree, N.; Garver, J.; Dickens, B.; Finn, T.; Martin, J. Accelerated UV weathering device based on integrating sphere technology. Rev. Sci. Instrum. 2004, 75, 4951-4959.

64. Chen, W.; Auad, M.L.; Williams, R.J.J.; Nutt, S.R. Improving the dispersion and flexural strength of multiwalled carbon nanotubes-stiff epoxy composites through beta-hydroxyester surface functionalization coupled with the anionic homopolymerization of the epoxy matrix. Eur. Polym. J. 2006, 42, 2765-2772.

65. Kashiwagi, T.; Grulke, E.; Hilding, J.; Groth, K.; Harris, R.; Butler, K.; Shields, J.; Kharchenko, S.; Douglas, J. Thermal and flammability properties of polypropylene/carbon nanotube nanocomposites. Polymer 2004, 45, 4227-4239.

66. Kashiwagi, T.; Du, F.M.; Douglas, J.F.; Winey, K.I.; Harris, R.H.; Shields, J.R. Nanoparticle networks reduce the flammability of polymer nanocomposites. Nat. Mater. 2005, 4, 928-933.

67. Kashiwagi, T.; Mu, M.; Winey, K.; Cipriano, B.; Raghavan, S.R.; Pack, S.; Rafailovich, M.; Yang, Y.; Grulke, E.; Shields, J.; Harris, R.; Douglas, J. Relation between the viscoelastic and flammability properties of polymer nanocomposites. Polymer 2008, 49, 4358-4368.

68. Schartel, B.; Pötschke, P.; Knoll, U.; Abdel-Goad, M. Fire behaviour of polyamide 6/multiwall carbon nanotube nanocomposites. Eur. Polym. J. 2005, 41, 1061-1070.

69. Verdejo, R.; Barroso-Bujans, F.; Rodriguez-Perez, M.A.; Saja, J.A.d.; Arroyo, M.; Lopez-Manchado, M.A. Carbon nanotubes provide self-extinguishing grade to silicone-based foams. J. Mater. Chem. 2008, 18, 3933-3939.

70. Kim, J.Y.; Park, H.S.; Kim, S.H. Thermal decomposition behavior of carbon-nanotubereinforced poly(ethylene 2,6-naphthalate) nanocomposites. J. Appl. Polym. Sci. 2009, 113, 2008-2017.

71. Fu, S.; Song, P.; Yang, H.; Jin, Y.; Lu, F.; Ye, J.; Wu, Q. Effects of carbon nanotubes and its functionalization on the thermal and flammability properties of polypropylene/wood flour composites. J. Mater. Sci. 2010. 45, 3520-3528.

72. Zammarano, M.; Krämer, R.H.; Harris, R.; Ohlemiller, T.J.; Shields, J.R.; Rahatekar, S.S.; Lacerda, S.; Gilman, J.W. Flammability reduction of flexible polyurethane foams via carbon nanofiber network formation. Polym. Adv. Technol. 2008, 19, 588-595.

73. Nyden, M.R.; Harris, R.H.; Kim, Y.S.; Davis, R.D.; Marsh, N.D.; Zammarano, M. Characterizing particle emissions from burning polymer nanocomposites. Tech. Proc. 2010 NSTI Nanotechnol. Conf. Expo 2010, 1, 717-719.

74. Uddin, N.; Nyden, M.R.; Davis, R.D. Characterization of Nanoparticle Release from Polymer Nanocomposites Due to Fire. In Proceedings of the Nanotech 2011 Conference and Expo, Boston, MA, June 13-16, 2011. 
75. Dittrich, B.; Wartig, K.A.; Hofmann, D.; Mülhaupt, R.; Schartel, B. Carbon black, multiwall carbon nanotubes, expanded graphite and functionalized graphene flame retarded polypropylene nanocomposites. Polym. Adv. Technol. 2013, 24, 916-926.

76. Bouillard, J.; R'Mili, B.; Moranviller, D.; Vignes, A.; Le Bihan, O.; Ustache, A.; Bomfim, J.S.; Frejafon, E.; Fleury, D. Nanosafety by design: risks from nanocomposite/nanowaste combustion. J. Nanoparticle Res. 2013, 15, 1-11.

77. Coleman, J.N.; Cadek, M.; Blake, R.; Nicolosi, V.; Ryan, K.P.; Belton, C.; Fonseca, A.; Nagy, J.B.; Gun'ko, Y.K.; Blau, W.J. High Performance Nanotube-Reinforced Plastics: Understanding the Mechanism of Strength Increase. Adv. Funct. Mater. 2004, 14, 791-798.

78. Cai, H.; Yan, F.Y.; Xue, Q.J. Investigation of tribological properties of polyimide/carbon nanotube nanocomposites. Mater. Sci. Eng. a-Struct. Mater. Prop. Microstruct. Process. 2004, 364, 94-100.

79. Gupta, A.; Choudhary, V. Thermal and mechanical properties of poly(trimethyelene terephthalate)/acid-treated multiwalled carbon nanotube composites. J. Mater. Sci. 2013. 48, 7063-7070.

80. Seyhan, A.T.; Tanoglu, M.; Schulte, K. Tensile mechanical behavior and fracture toughness of MWCNT and DWCNT modified vinyl-ester/polyester hybrid nanocomposites produced by 3-roll milling. Mater. Sci. Eng. a-Struct. Mater. Prop. Microstruct. Process. 2009, 523, 85-92.

81. Zhu, J.; Kim, J.D.; Peng, H.Q.; Margrave, J.L.; Khabashesku, V.N.; Barrera, E.V. Improving the dispersion and integration of single-walled carbon nanotubes in epoxy composites through functionalization. Nano Lett. 2003, 3, 1107-1113.

82. Gojny, F.H.; Nastalczyk, J.; Roslaniec, Z.; Schulte, K. Surface modified multi-walled carbon nanotubes in CNT/epoxy-composites. Chem. Phys. Lett. 2003, 370, 820-824.

83. Mueller, N.C.; Buha, J.; Wang, J.; Ulrich, A.; Nowack, B. Modeling the flows of engineered nanomaterials during waste handling. Environ. Sci. Process. Impacts 2013, 15, 251-259. 


\title{
High Strength and High Modulus Electrospun Nanofibers
}

\author{
Jian Yao, Cees W. M. Bastiaansen and Ton Peijs
}

Abstract: Electrospinning is a rapidly growing polymer processing technology as it provides a viable and simple method to create ultra-fine continuous fibers. This paper presents an in-depth review of the mechanical properties of electrospun fibers and particularly focuses on methodologies to generate high strength and high modulus nanofibers. As such, it aims to provide some guidance to future research activities in the area of high performance electrospun fibers.

Reprinted from Fibers. Cite as: Yao, J.; Bastiaansen, C.W.M.; Peijs, T. High Strength and High Modulus Electrospun Nanofibers. Fibers 2014, 2, 158-186.

\section{Introduction}

Over the last two decades, a relatively old technique, electrospinning, has been receiving renewed interest due to its huge potential of producing polymer nanofibers [1-3]. Since then, basic electrospinning theories [4-6] have been developed and hundreds of electrospun nanofibers from different polymer systems have been practically produced [7-9].

The small fiber diameter (usually $20 \mathrm{~nm}-1 \mu \mathrm{m}$ ), high specific surface area (tens to hundreds $\mathrm{m}^{2} / \mathrm{g}$ ), high porosity and small pore size renders electrospun nanofibers of interest for a wide range of potential applications in tissue engineering, drug delivery, textiles, filtration, composite reinforcements, etc. [10-12]. Many applications require however high mechanical property nanofibers and for applications like air filtration systems [13], microfibers are still employed as supports for electrospun fibers as these nanofibers are often too weak to be used independently.

Compared to corresponding textile fibers made from the same polymers, the mechanical properties of electrospun nanofibers are often found to be poor. Electrospun nanofibers typically display tensile strengths below $300 \mathrm{MPa}$ and Young's moduli below $3 \mathrm{GPa}[12,14-19]$, which can be mainly ascribed to the low degree of orientation and chain extension of the polymer chains along the fiber axis. The objective of this review article is to report on the various research works on mechanical properties of electrospun nanofibers and to answer the question whether it is at all possible to produce high modulus and high strength electrospun nanofibers. For this, first a brief overview of different concepts to produce conventional high performance synthetic fibers will be given, focusing on flexible 
chain versus rigid chain polymers. Subsequently, we will discuss current progresses in the manufacturing of high performance electrospun nanofibers.

\section{High Strength and High Modulus Fibers}

\subsection{Basic Concepts for High Performance Fibers}

A century ago, natural fibers like cotton, silk, and wool dominated the market due to their abundance and aesthetic appeal. In the 1920s, Hermann Staudinger was the first to propose the concept of macromolecules [20] and this epoch-making idea greatly influenced the discovery of synthetic fibers with the first synthetic fiber being invented in 1935 by Wallace Carothers, which is known to us as polyamide (PA) or nylon fiber [21].

The two most commonly used aliphatic polyamide fibers are polyamide 6 and polyamide 6,6 which are made of caprolactam and hexamethylenediamine with adipic acid, respectively. Polyamide (PA) fibers are produced by melt-spinning and are given moderate molecular orientation and crystallinity after post-drawing. Molecular orientation and crystallinity, along with hydrogen bonding between chains provided by the amide group (-NH-CO-) [22] provides them with good mechanical properties and abrasion resistance, which renders them to be one of the most widely used industrial fibers. Polyethylene terephthalate (PET), which is the most important commercial polyester, first appeared on the market in 1953 [23] and is now fully established in textile and technical fibers. PET is produced by polymerization of either dimethyl terephthalate or terephthalic acid with ethylene glycol. Melt-spinning in combination post-drawing is also applied in the manufacture of PET fibers, with main applications in textiles, ropes, tyres, carpets, and so on. Since these initial developments on PA and PET, more and more synthetic fibers have been investigated and developed.

The tensile strength and Young's modulus of those traditional synthetic fibers are usually limited to below $1 \mathrm{GPa}$ and $15 \mathrm{GPa}$, respectively. The relatively low mechanical performance of these textile fibers greatly limits their applications in areas such as aerospace, protective clothing, armor, advanced composites, etc.

In 1932, Staudinger [24] postulated the basic requirements for producing a high strength and high modulus synthetic fiber. In his structural model for such a fiber, all molecular chains should be fully extended and perfectly aligned along the fiber axis. Simultaneously, few chain end defects should exist as the tensile strength is determined by secondary bonds rather than primary bonds. The fully extended and oriented polymer chains will provide ultimate stiffness to the fiber while the few chain end defects as in the case of high molecular weight polymers provides high tenacity, the combination of both making the ideal fiber (Figure 1). 


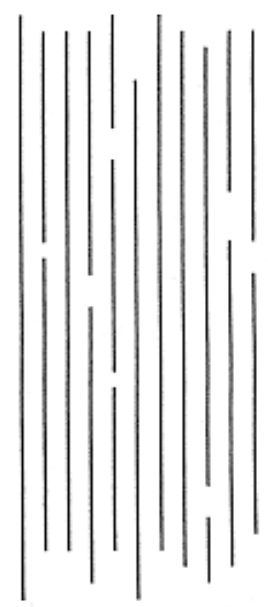

Figure 1. Ideal polymer chains model for producing high strength and high modulus fibers.

It has long been recognized by Meyer and Lotmar [25] that only extended polymer chains will provide high stiffness. In 1960, Terloar [26] estimated the longitudinal modulus of a single extended polyethylene chain to be $182 \mathrm{GPa}$ in comparison to the Young's modulus of isotropic polyethylene being less than $2 \mathrm{GPa}$ [27]. More calculations showed that the Young's modulus of a single extended polyethylene chain can reach even higher values up to $320 \mathrm{GPa}$ [28]. On the other hand, polymers like polypropylene with a helical chain configuration, exhibit a much lower theoretical modulus compared with planar zig-zag configurations as in polyethylene. Elastic modulus of crystalline regions in the direction parallel to the chain axis have since also been measured experimentally using $X$-ray diffraction (Table 1), providing great insight into the potential of certain polymers to generate high performance fibers [29-31].

All the above studies indicated that high modulus and high strength in fibers mainly relies on high polymer chain orientation and extension, combined with sufficient chain length. Inspired by this, polymer scientists have attempted to improve the mechanical properties of fibers by pursuing two routes to reach Staudinger's ideal model, i.e., flexible chain polymers and rigid chain polymers [32]. 
Table 1. Theoretical crystal modulus of polymers derived from X-ray diffraction studies [29-31].

\begin{tabular}{cc}
\hline Polymer & Theoretical modulus (GPa) \\
\hline Polyethylene (PE) & 235 \\
Poly(vinyl alcohol) (PVA) & 250 \\
Polyamide-6 (PA 6) & 157 \\
Poly(ethylene terephthalate) (PET) & 108 \\
Polypropylene (PP) & 40 \\
Polyacrylonitrile (PAN) & $35-55$ \\
Thermotropic polyester (Vectran) & 126 \\
Poly(p-phenylene terehthalamide) (PPTA) & 156 \\
Poly(phenylene benzobisoxazole) (PBO) & 478 \\
\hline
\end{tabular}

\subsection{High Performance Fibers Based on Flexible Polymer Chains}

In the case of flexible chains, notably polyethylene, the chains tend to fold upon crystallization and in order to get the desired level of chain extension necessary to exploit the intrinsic properties of the polymer chain, solid-state drawing at elevated temperature but below the melting temperature is applied [33-35]. Great efforts have been devoted to melt-spinning and later also solution-spinning of polyethylene in the 1970s. Ward et al. [36,37] developed polyethylene fibers using a process of melt-spinning followed by drawing in the solid state. This technique had limitations with respect to the use of high molecular weight polymers and as such the tenacity of the obtained fibers, as spinnability (as a result of the rapidly increasing melt viscosity) and fiber drawability both decreased with increasing molecular weight. An initial breakthrough in the development of high strength polyethylene fibers was the so-called surface growth technique from Zwijnenberg and Pennings [38,39]. Here, Young's moduli exceeding $100 \mathrm{GPa}$ and tensile strengths over $3 \mathrm{GPa}$ were obtained from ultra-high molecular weight polyethylene. Their results were a break-through as this was the first experimental evidence that high modulus and high strength structures could be produced from flexible chain polymers [40].

An industrial breakthrough in the production of high modulus and high strength polymer fibers was achieved by the solution (or gel) spinning process developed at DSM in the Netherlands at the end of the 1970s. Smith and Lemstra [41-44] discovered that as-spun ultra-high molecular weight polyethylene filaments from solution could be hot-stretched in the solid-state below the melting temperature to very high draw ratios. In the gel-spinning process, a semi-diluted ultra-high molecular weight $\left(\mathrm{Mw}>10^{6} \mathrm{~g} / \mathrm{mol}\right)$ polyethylene solution of low polymer concentration is squeezed through a spinneret and after quenching in a water bath a gel-like filament is obtained (Figure 2). This results in a morphology with a low 
entanglement density of polymer chains in the as-spun gel-like fiber, which renders them super-drawable.

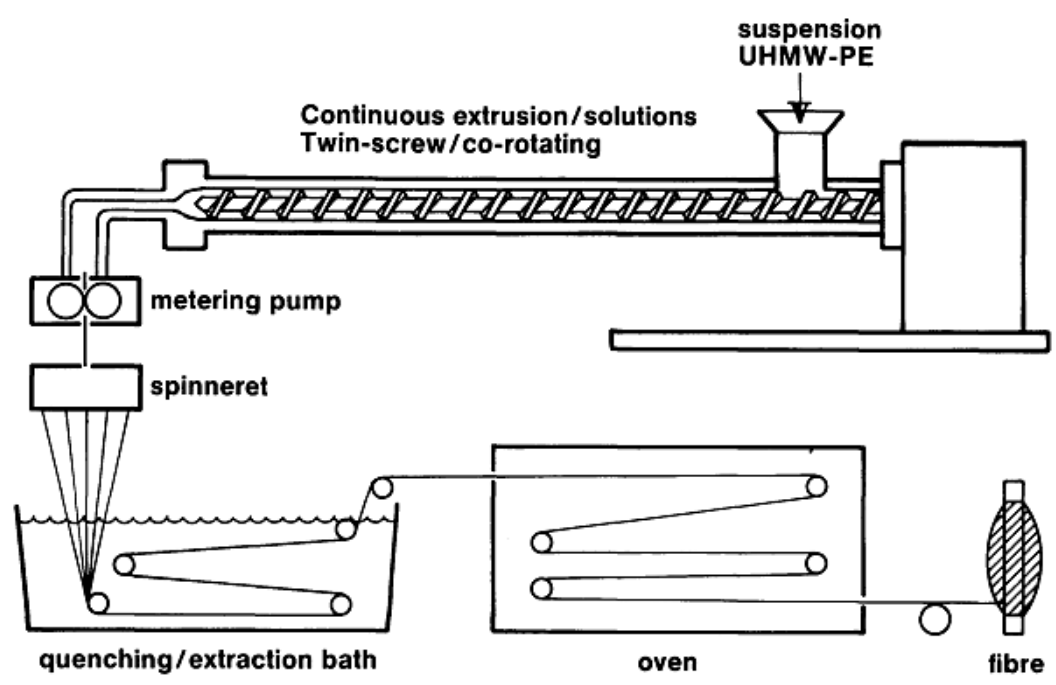

Figure 2. Schematics of the gel-spinning process [34].

Upon solid-state drawing, the lamellae structure of the as-spun polyethylene fiber will initially orient in the drawing direction. At higher draw-ratios, these lamellae ideally unravel into chain-extended structures approaching ultimate properties (see Table 1). In fact, the solid-state drawing step can be regarded as the single most important process step needed to create high performance fibers, based on flexible chain polymers. Since the relaxation times of flexible chain polymers are typically very short, the elongation flow induced orientation in the as-spun fibers rapidly disappears due to relaxation before solidification. High performance fibers based on flexible chain polymers are therefore typically post-drawn in the solid-state below the melting temperature as this will prevent chain relaxation after orientation and chain extension.

Dyneema ${ }^{\circledR}$ by DSM and Spectra ${ }^{\circledR}$ by its licensee Allied Signal (now Honeywell) are two commercially available high strength and high modulus fibers that use ultra-high molecular weight polyethylene (UHMWPE) as a starting material. These fibers have Young's moduli exceeding $100 \mathrm{GPa}$ and tensile strengths of more than $3 \mathrm{GPa}$, i.e., 100 times that of bulk polyethylene. In combination with their low density $\left(<1000 \mathrm{~kg} / \mathrm{m}^{3}\right)$, this leads to exceptionally high specific mechanical properties (properties per unit weight), making these UHMWPE fibers of interest for a wide range of applications ranging from maritime ropes to protective gloves, bullet-proof vests, and other advanced composites applications. 


\subsection{High Performance Fibers Based on Rigid Polymer Chains}

The discovery of rigid rod polymers to produce high performance fibers originated from the discovery of para-oriented aromatic polyamide [45,46], notably, poly ( $p$-phenylene terehthalamide) (PPTA). Large research efforts led to significant advances in the fundamental understanding of PPTA polymerization [47], spinning solutions [48,49], spinning process and the structure of para-aramid fibers $[48,50]$, taking full advantage of its rigid rod like molecular structure and unique liquid crystalline properties.

Generally, PPTA exhibits lyotropic liquid crystalline properties in proper solvents, viz. concentrated sulphuric acid. At low concentrations, the rod-like molecules are randomly distributed but they tend to order and form nematic domains above a certain concentration (see also Section 3.3.1.). As chain extension in rigid chains is already built in by the chemist, it is not essential to post-draw these as-spun filaments, which is the main difference between processing fibers based on rigid and flexible chain molecules.

The melting point of PPTA is higher than its degradation temperature, so melt-spinning is not feasible in the case of PPTA. Dry-jet (air gap) wet spinning [47] was utilized as a novel spinning route for these materials in the 1970s. Anisotropic solutions with concentrations of around 19-20 PPTA in concentrated sulphuric acid at 70-90 ${ }^{\circ} \mathrm{C}$, i.e., concentrated solutions of moderate viscosities, were used for spinning of these fibers. Orientation with extended chain configuration of the liquid crystalline domains is achieved in the air-gap (Figure 3) and fixed in the coagulation bath usually made up of water or diluted sulphuric acid with temperatures in the range of $0-5{ }^{\circ} \mathrm{C}$. The as-spun fibers are then washed, neutralized, and dried afterwards. The degree of chain orientation and crystallinity can be further tuned by changing the draw ratio during spinning and/or by heat treatments.

Para-aramid fibers are manufactured under the trademark of Kevlar ${ }^{\circledR}$ (Du Pont, Wilmington, USA) and Twaron ${ }^{\circledR}$ (Teijin Aramid, Arnhem, The Netherlands). A combination of properties like light weight, high strength, high modulus, excellent high temperature resistance and good resistance to chemicals, make para-aramid fibers of interest for aerospace composites, anti-ballistic materials, and many other advanced applications.

Another high strength and high modulus fiber based on (semi) rigid polymer chains is aromatic polyester which exhibits liquid crystalline properties. This thermoptropic liquid crystalline polymer (TLCP) fiber was initially developed by Celanese Corporation and became commercially available in the mid-1980s under the trade name Vectran ${ }^{\circledR}$. Melt-spinning using conventional extrusion practices in combination with moderate draw-down is performed on these thermotropic liquid crystal polyesters to fully exploit its liquid crystalline properties [51]. 


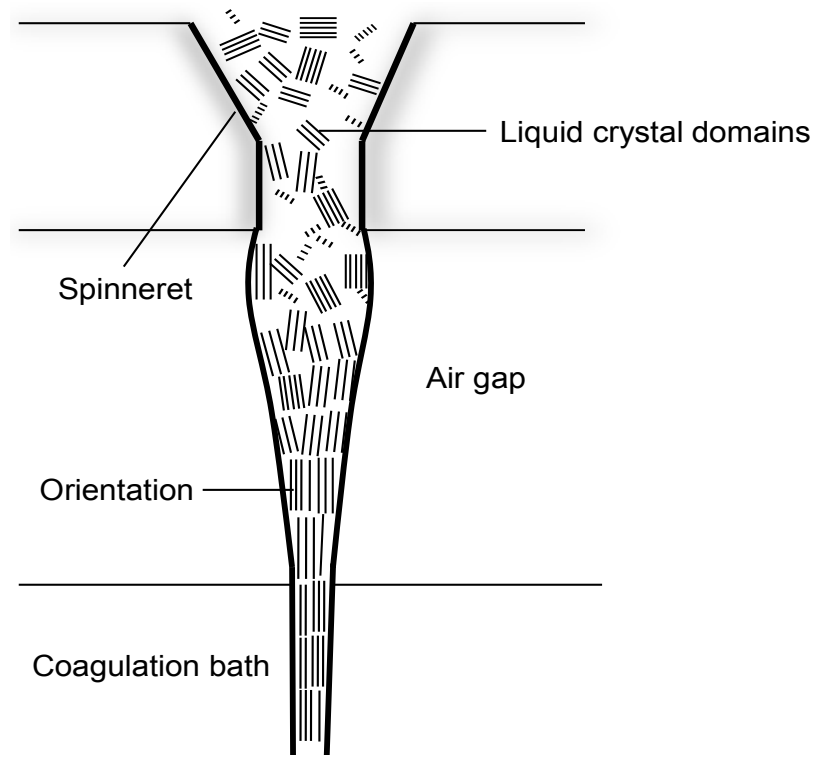

Figure 3. Molecular orientation during dry-jet wet spinning of PPTA.

Other more recent developments in high performance fibers based on lyotropic liquid crystalline rigid rod polymers are poly(phenylene benzobisoxazole) (PBO) from Toyobo Corporation under the trade name Zylon ${ }^{\circledR}$ [52-54] and poly(hydroquinone-diimidazopyridine) ("M-5") [55] from Akzo-Noble with a very similar chemical structure as $\mathrm{PBO}$ but exhibiting much better compressive properties [56]. The mechanical properties of all these fibers are later shown in Figure 21.

\section{Electrospun Nanofibers}

\subsection{Basic Concepts of Electrospinning}

Electrospinning is a versatile technique that makes use of an -in principlevery simple experimental set-up. Normally, polymers or polymer mixtures to be used in electrospinning are dissolved in organic solvents to make homogenous spinnable solutions. These spinning solutions are usually pumped from a single nozzle at a controlled feeding rate. $10-50 \mathrm{kV}$ DC high voltages are typically applied between two electrodes within a distance of $10-30 \mathrm{~cm}$ to generate an electrostatic field. Consequently, a pending droplet will be stretched to a Taylor cone first by electrostatic repulsion forces resulting from charges on the solution [57]. A solution jet will be ejected from the deformed cone when the repulsion force exceeds the surface tension of the pending droplet. In its flight to the counter electrode, it will 
move in a straight line for a short distance followed by a whipping path (Figure 4) accompanied with solvent evaporation and jet stretching until a solid nanofiber mat (Figure 5) is collected on the substrate.

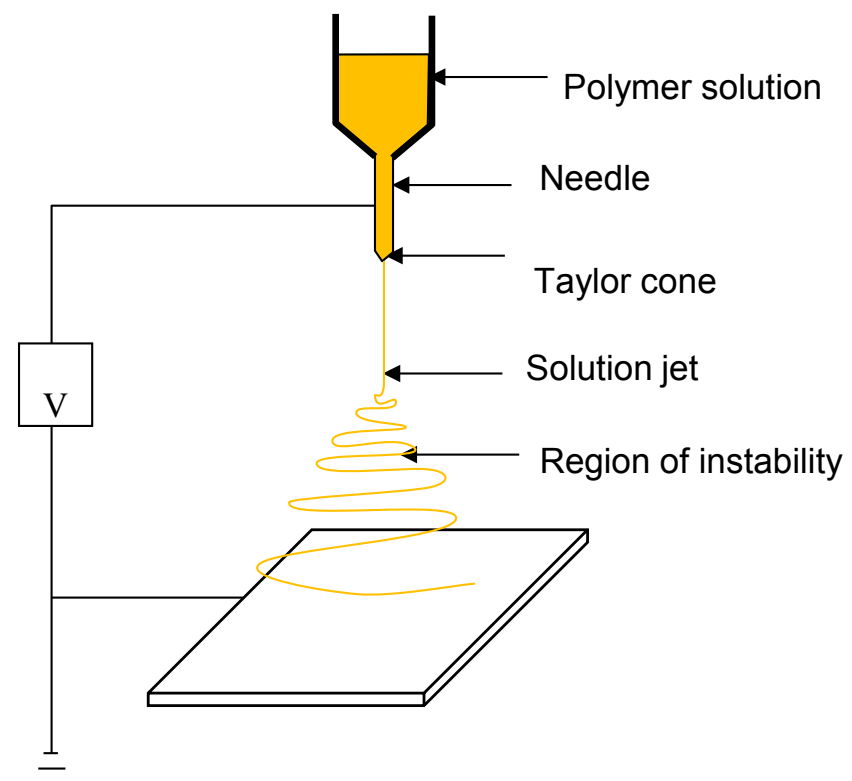

Figure 4. A typical electrospinning set-up with a grounded collector.
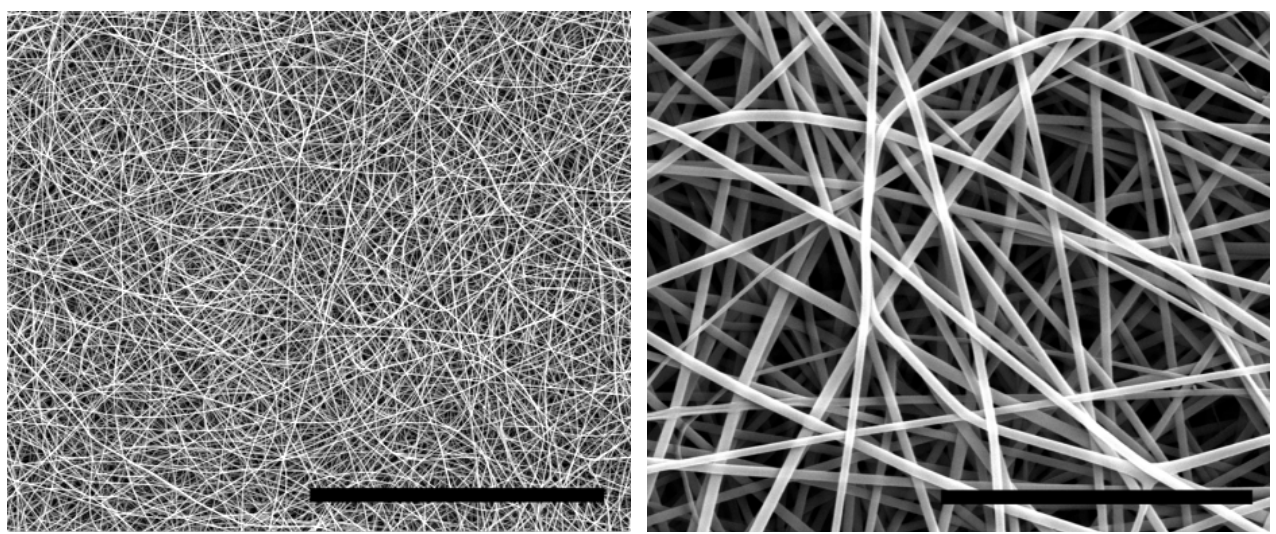

Figure 5. Two scanning electron microscope (SEM) pictures of an electrospun PA6 nanofiber non-woven mat under different magnifications (scale bars of $40 \mu \mathrm{m}$ and $5 \mu \mathrm{m}$, respectively). 
In principle, a numbers of parameters, which can be classified into solution and processing parameters, are believed to affect the electrospinning process. Solution parameters include viscosity (Figure 6), molecular weight of polymer, conductivity, surface tension, etc. Solution feeding rate, process temperature and humidity, applied voltage, distance between electrodes, and design of collector are typical processing parameters which also influence spinnability and fiber morphology [58].

A significant disadvantage of electrospinning as a means to create polymer nanofibers has been the low production rate using single needle systems, which have been typically restricted to a few grams per hour. However, recently, several new technologies have been suggested that tackle this issue.

Free liquid surface electrospinning [59] such as the Nanospider ${ }^{\circledR}$ technology applies a nozzle-less electrospinning head instead of a traditional nozzle based set-up. Its rotating electrospinning head can carry a thin layer of polymer solution from a liquid polymer bath and nanofiber layers in a high throughout rate can be produced when the polymer solution is exposed to a high electrical field (Figure 7a). Electrospinning has also been combined with traditional polymer processing techniques such as twin-screw extrusion in order to improve the ability to spin more viscous systems including polymer melts [60]. Moreover, such a process can generate high production rates when combined with multi-nozzle spinnerets (Figure 7b) [61].
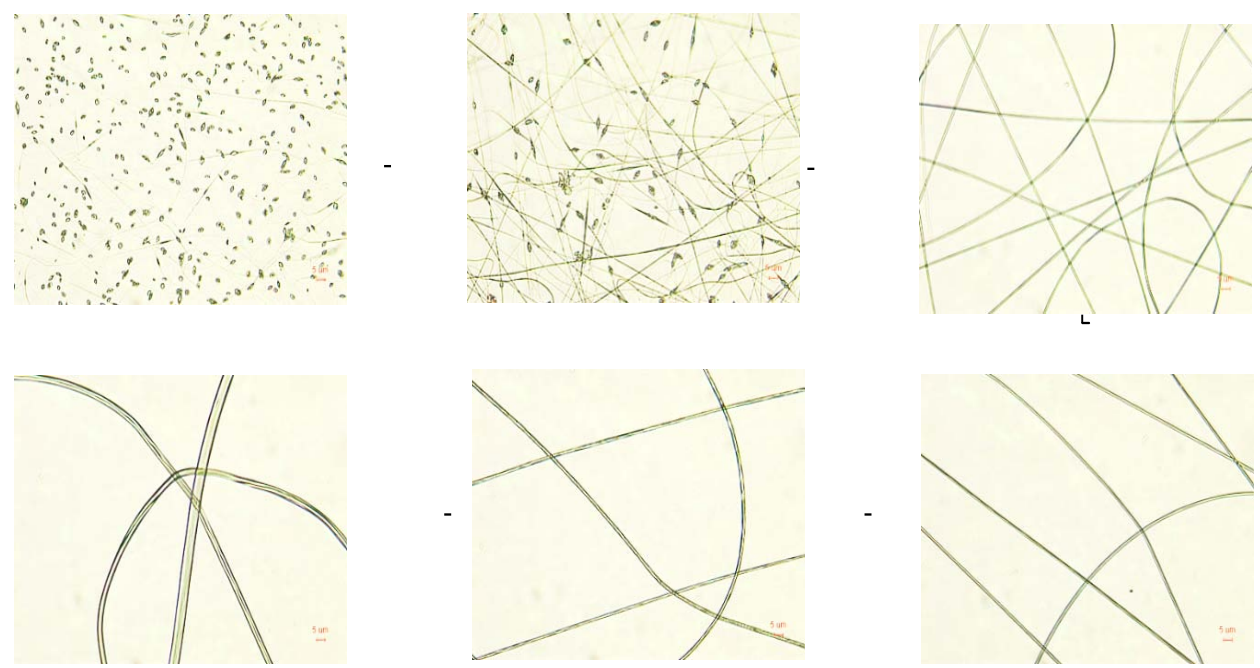

Figure 6. Different fiber morphologies of electrospun nanofibers produced from low solution concentration to high solution concentration of poly(methyl methacrylate) (PMMA) in DMF. 
Recently also alternative spinning technologies for nanofibers have been introduced, such as a rotary jet-spinning technology that is capable of high rate spinning [62]. Unlike conventional electrospinning using an electrostatic force as driving force to generate fibers, in rotary jet-spinning nanofibers are fabricated by a centrifugal force caused by high speed rotation of a polymer solution or melt as shown in Figure 7c.

(a)

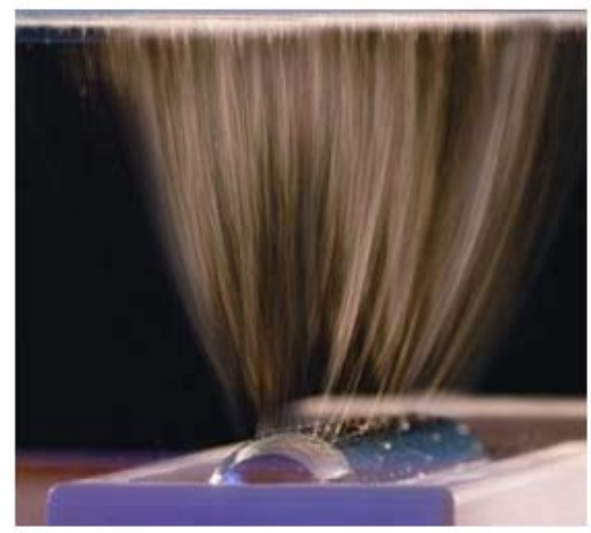

(b)

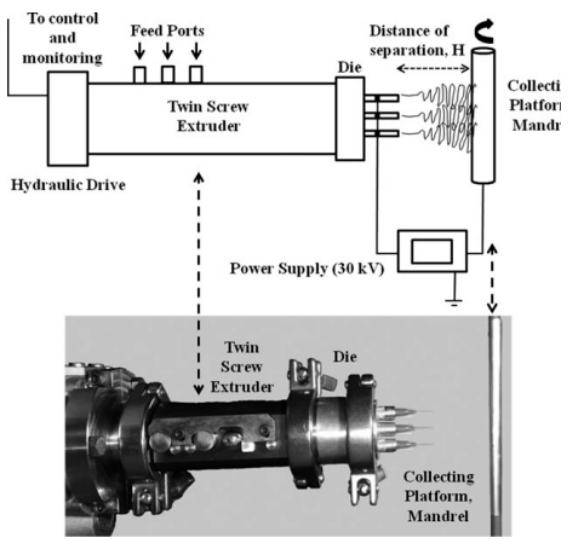

(c)

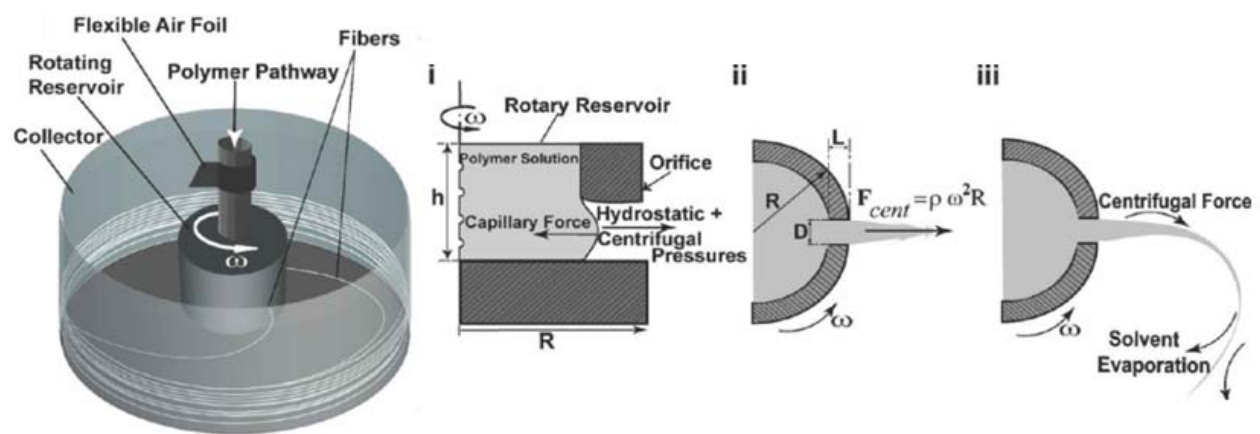

Figure 7. Novel nanofiber production technologies (a) nozzle-less electrospinning with a rotating head [59]. Reproduced with permission from Petrik et al., 2009. Copyright Cambridge Univ Press. (b) Multi-nozzle electrospinning with a twin-screw extrusion [61]. Reproduced with permission from Senturk-Ozer et al., 2012. Copyright John Wiley \& Sons. (c) Rotary jet-spinning [62]. Reproduced with permission from Badrossamay et al., 2010. Copyright American Chemical Society.

To make high performance electrospun nanofibers, in principle, the molecular structures of nanofibers should be oriented with chain extension, and should 
resemble the ideal polymer chains model described in Figure 1. In the next section, recent reports on mechanical properties of electrospun nanofibers are presented according to their intrinsic chain flexibility, viz. flexible chains versus rigid chains. Some nanofibers possessing enhanced mechanical properties are highlighted.

\subsection{Electrospun Nanofibers Based on Flexible Chain Polymers}

Inspired by the success of Dyneema ${ }^{\circledR}$, Rein et al. [63] tried to fabricate UHMWPE fibers using the electrospinning method. The mechanical properties of manually twisted yarn from as-spun nanofiber mats were investigated and a tensile strength of $129 \mathrm{MPa}$ and modulus of $0.4 \mathrm{GPa}$ were reported, which are well below those of commercial UHMWPE fibers at around $3000 \mathrm{MPa}$ and $100 \mathrm{GPa}$, respectively [64]. The relatively low strength and modulus of these electrospun UHMWPE fibers can to some extent be explained from their wide-angle X-ray scattering (WAXS) pattern (Figure 8). The WAXS data shows a significant difference of the average molecular chain orientation in electrospun and solution-spun ultra-drawn UHMWPE. Where electrospun UHMWPE shows broad (110) and (200) reflection arcs, shows only ultra-drawn UHMWPE greatly intensified reflections typical of a highly oriented polymer fiber.

(a)

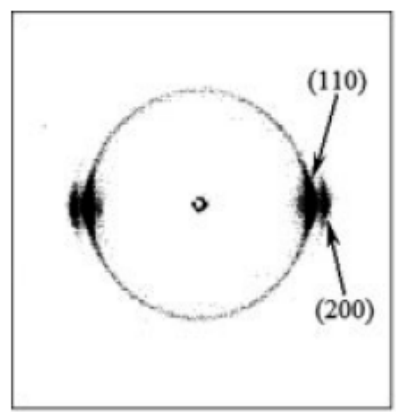

(b)

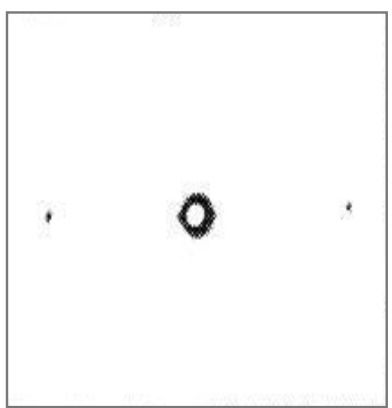

Figure 8. (a) WAXS pattern of electrospun UHMWPE nanofiber [63], showing broad reflection arcs typical of a moderately oriented polymer structure. Reproduced with permission from Rein et al., 2007. Copyright John Wiley \& Sons. (b) WAXS pattern of solution-spun ultra-drawn UHMWPE fiber with draw-ratio 100, showing intense reflections typical of a highly oriented polymer structure [33].

With respect to the vast amount of research on other electrospun nanofibers based on flexible chain polymers such as aliphatic polyamide and polyester, a relatively limited number of studies involved the mechanical properties of non-woven nanofiber mats or even single nanofibers. According to Table 2, the 
mechanical properties of most electrospun polyamide and polyester fibers are also not comparable with conventional microfibers manufactured by melt-spinning, which again can be ascribed to the low degree of chain orientation in these as-spun nanofibers and the absence of a post-drawing step in the electrospinning process. In fact, in many cases the properties of electrospun fiber properties are even inferior to that of the bulk polymer, which can be attributed to factors, such as residual solvent, plasticization, and porosity.

Table 2. Mechanical properties of electrospun polyamide (PA) and poly(ethylene terephthalate) (PET) nanofibers.

\begin{tabular}{|c|c|c|c|c|c|}
\hline Polymer & Solvent \& concentration & Sample & $\begin{array}{l}\text { Modulus } \\
\text { (MPa) }\end{array}$ & $\begin{array}{l}\text { Strength } \\
\text { (MPa) }\end{array}$ & Refs. \\
\hline PA 6 & $\begin{array}{c}6 \mathrm{wt} \% \text { in } \\
1,1,1,3,3,3 \text {-hexafluoro-2- } \\
\text { propanol }\end{array}$ & $\begin{array}{c}\text { Nonwoven } \\
\text { nanofiber mat }\end{array}$ & $34 \pm 2$ & $7.2 \pm 0.5$ & [33] \\
\hline PA 6,6 & $\begin{array}{c}7.5 \mathrm{wt} \% \text { in } \\
1,1,1,3,3,3 \text {-hexafluoro-2- } \\
\text { propanol }\end{array}$ & $\begin{array}{c}\text { Nonwoven } \\
\text { nanofiber mat }\end{array}$ & $21 \pm 1$ & $6.5 \pm 0.8$ & \\
\hline \multirow[t]{2}{*}{ PA 6} & \multirow[t]{2}{*}{$20 \mathrm{wt} \%$ in formic acid } & $\begin{array}{c}\text { Nonwoven } \\
\text { nanofiber mat }\end{array}$ & 19 & 10.5 & \multirow[t]{2}{*}{ [33] } \\
\hline & & $\begin{array}{c}\text { Single } \\
\text { nanofiber }\end{array}$ & 902 & 304 & \\
\hline PA 6,6 & $20 \mathrm{wt} \%$ in formic acid & $\begin{array}{c}\text { Single } \\
\text { nanofiber }\end{array}$ & $950 \pm 390$ & $150 \pm 49$ & [33] \\
\hline PA 6,6 & $\begin{array}{l}10 w t \% \text { in formic acid \& } \\
\text { chloroform }(75: 25 v / v)\end{array}$ & $\begin{array}{l}\text { Nanofiber } \\
\text { yarn }\end{array}$ & 1216 & 120 & [33] \\
\hline PA 6 & $\begin{array}{l}12 w t \% \text { in formic acid \& } \\
\text { acetic acid }(50: 50 w / w)\end{array}$ & $\begin{array}{l}\text { Single } \\
\text { nanofiber }\end{array}$ & $1320 \pm 152$ & $78.1 \pm 6.0$ & [33] \\
\hline PA 6 & $\begin{array}{l}12 \mathrm{wt} \% \text { in formic acid \& } \\
\text { acetic acid }(50: 50 w / w)\end{array}$ & $\begin{array}{c}\text { Nonwoven } \\
\text { nanofiber mat }\end{array}$ & $418 \pm 93$ & $57.7 \pm 8.9$ & [33] \\
\hline PET & $\begin{array}{c}30 \%(w / v) \text { in TFA \& DCM } \\
(70: 30 v / v)\end{array}$ & $\begin{array}{c}\text { Nonwoven } \\
\text { nanofiber mat }\end{array}$ & 60 & 3.7 & [33] \\
\hline PA $6 / 6,6$ & Melt & Bulk & $2000-2500$ & $50-80$ & \multirow{2}{*}[33]{} \\
\hline PET & Melt & Bulk & $2000-3000$ & $50-150$ & \\
\hline PA $6 / 6,6$ & Melt-spun + drawn & Single fiber & 6000 & 1000 & \multirow{2}{*}{ [33] } \\
\hline PET & Melt-spun + drawn & Single fiber & 15000 & 1100 & \\
\hline
\end{tabular}

Although traditional methods to induce high molecular orientation like post-drawing are usually not feasible for single nanofibers, at least for the time being due to technological difficulties, limited stretching or drawing has been attempted to oriented nanofiber mats in order to generate improved molecular orientation and crystallinity. Zong et al. [72] stretched PLGA nanofiber membranes up to a deformation of $450 \%$ at $90{ }^{\circ} \mathrm{C}$. An improvement in mechanical properties after post-drawing was observed as seen in Figure 9, although the properties achieved remained low. Wu et al. [73] also tried solid-state hot-drawing at $135{ }^{\circ} \mathrm{C}$ of polyacrylonitrile (PAN) nanofiber sheets. Here, the crystallinity increased from $7.9 \%$ 
for as-spun nanofiber mats to $31.8 \%$ for hot-drawn mats, while the tensile strength was raised from $100 \mathrm{MPa}$ to $220 \mathrm{MPa}$, correspondingly.

Kongkhlang et al. [74] attempted to induce favorable orientation and increased crystallinity directly into as-spun electrospun nanofibers using a high-speed collector for nanofibers collection. Figure 10 exhibits two 2D wide-angle X-ray diffraction patterns of polyoxymethylene (POM) nanofibers electrospun with different take up speeds. It is clearly shown that a higher collecting speed of $1890 \mathrm{~m} / \mathrm{min}$ induces a higher degree of orientation in these nanofibers. In addition, it should be noted that alignment of the nanofibers within the mat is also vital for achieving high strength and high modulus structures as misalignment will significantly reduce the efficiency of the fibers within a mat and will greatly reduce its mechanical properties [75].

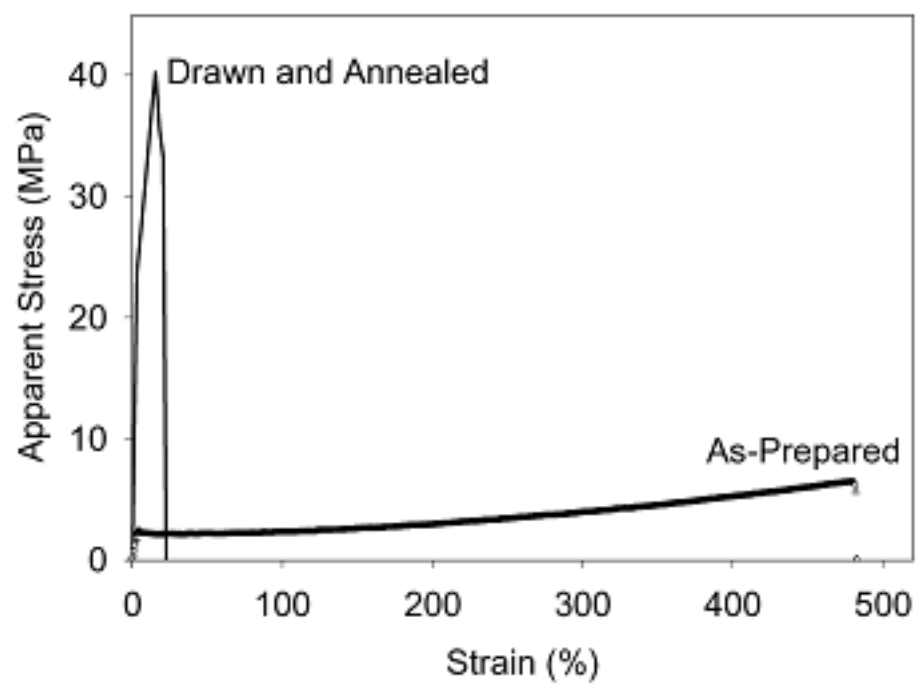

Figure 9. Strain-stress curves electrospun poly(glycolide-co-lactide) (PLGA) of as-spun nanofiber mat and nanofiber mat after a solid-state deformation of $450 \%$ [72]. Reproduced with permission from Zong et al., 2003. Copyright Elsevier Publisher.

Although it has been shown by various researchers that electrospinning can induce some level of chain orientation in fibers based on flexible chain polymers, these levels are often rather low. An interesting study in this respect was by Mohan et al. [76]. Here, small angle neutron scattering (SANS) was used to quantify the size and shape of the chain conformation in electrospun fibers of deuterated atactic polystyrene prepared from solutions. Although the orientation parameter $<\mathrm{P}_{2}>$ was found to increase with increasing collecting speed, the maximum value of about 0.15 (Figure 11a) was well below the orientation parameter expected for 
high performance polymer fibers with values typically approaching 1 . When the tangential velocity of the rotating collector was greater than the flight velocity of the fibers some degree of orientation of the polymer coils was induced. However, even at the highest collector speeds the ratio of the radii of gyration increased only by $20 \%$ from for bulk (17 nm) to fibers $(20 \mathrm{~nm})$, showing limited coil deformation. As the diameters of these fibers was much greater than the polystyrene radius of gyration these effects can be solely contributed to flow-induced orientation, excluding size or confinement effects as a result of nanosized fiber diameters.
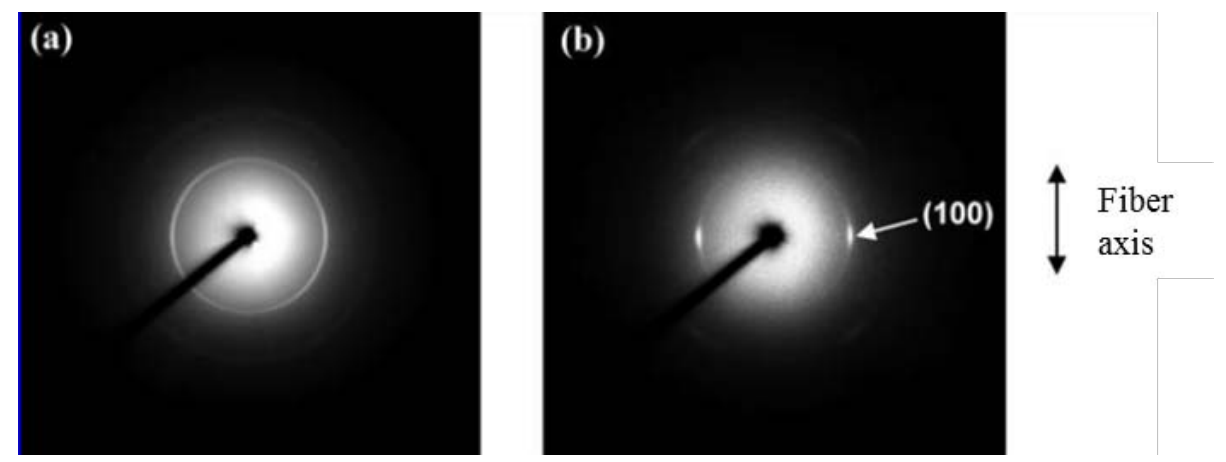

Figure 10. Two dimensional (2D) wide-angle X-ray diffraction patterns of electrospun polyoxymethylene (POM) nanofibers with different take up speeds (a) $630 \mathrm{~m} / \mathrm{min}$ (b) $1890 \mathrm{~m} / \mathrm{min}$ [74]. Reproduced with permission from Kongkhlang et al., 2008. Copyright Americal Chemical Society.

The influence of size effects on the mechanical properties of electrospun fibers has been studied extensively [77-83] with many studies showing an increase in Young's modulus with decreasing nanofiber diameter. In most cases it is assumed that such an increase is due to confinement of the polymer coils as they are forced to align along the nanofiber axis. Confinement effects are regarded by most researchers as the main reason for property improvement in electrospun nanofibers. However, although some degree of alignment and orientation can be envisaged with decreasing fiber diameter, particularly if the diameter is reduced to below the coil size, full chain extension as observed in super-drawn high performance fibers will be more difficult to achieve.

Nevertheless, a certain degree of orientation of polymer chains has been observed in electrospun fibers when their diameter is decreased. Figure 12a shows improved crystalline and extended amorphous structures in polycaprolactone (PCL) nanofibers for two diameters of $150 \mathrm{~nm}$ and $450 \mathrm{~nm}$, produced under similar conditions [78]. Although an increase in mechanical properties with decreasing fiber diameter was reported for these PCL fibers the maximum reported Young's 
modulus remained low at $2 \mathrm{GPa}$. Additionally, chain orientation has also been observed in nanofibers that showed birefringence under crossed polarizers $[8,84]$.
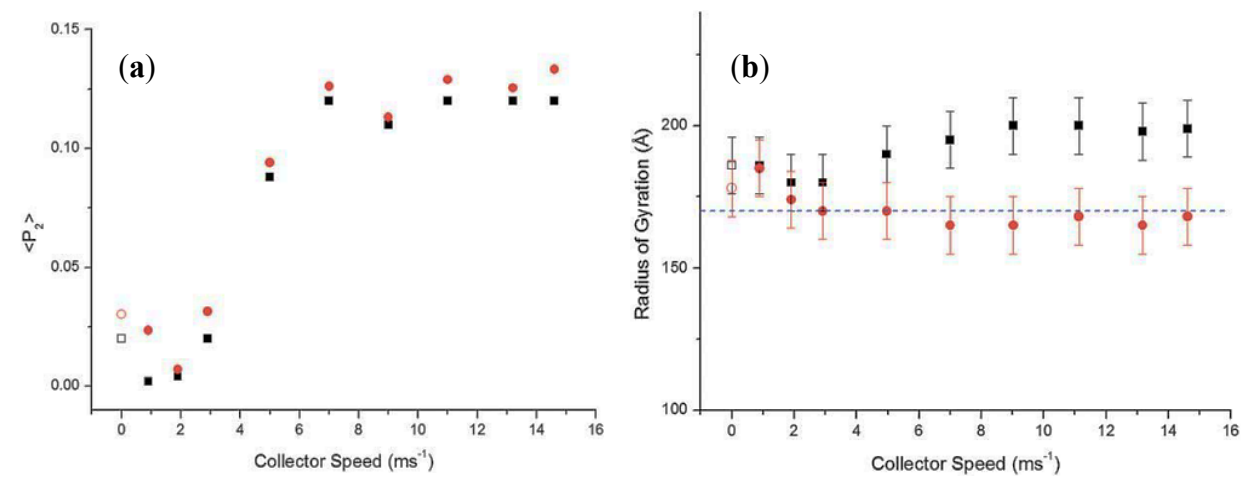

Figure 11. (a) Orientation parameter $<\mathrm{P}_{2}>$ obtained from SANS experiments for as-spun polystyrene fibers as a function of collector speed. Red circles represent uncorrected data for fiber angular alignment on the electrode while black squares are corrected data. Open symbols represent samples collected on static parallel plate electrode. (b) Radius of gyration of the polymer chains parallel (black squares) and perpendicular (red circles) to the fiber direction together with bulk data (dashed line) [76]. Reproduced with permission from Mohan et al., 2011. Copyright Royal Society of Chemistry.
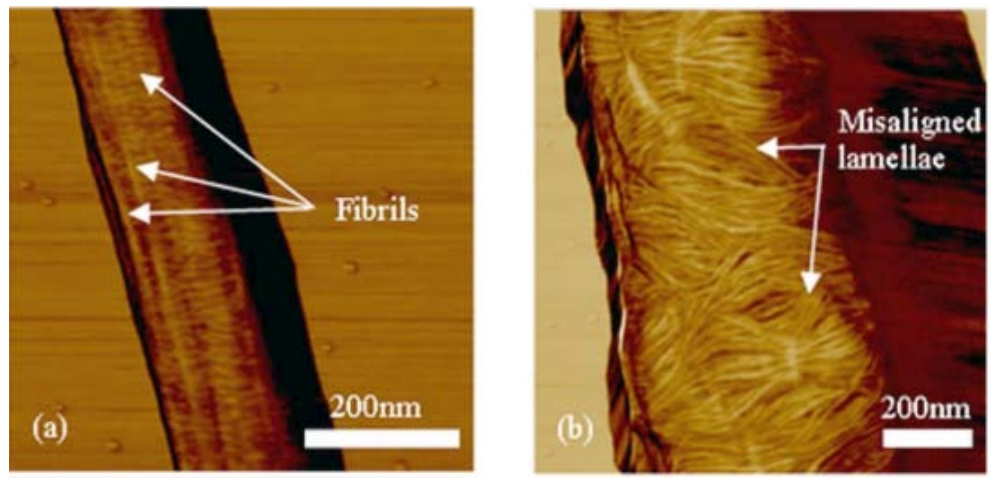

Figure 12. PCL nanofibers produced under similar conditions but with different fiber diameters (a) $150 \mathrm{~nm}$ (b) $450 \mathrm{~nm}$, showing a thinner nanofiber possessing a more aligned fibrillar and lamellae microstructure [78]. Reproduced with permission from Lim et al., 2008. Copyright AIP Publisher.

Stachewicz et al. [85] found that electrospun PVA nanofibers can possess a composite-like core-shell structure, with the shell region being aligned as a result of 
rapid solvent evaporation suppressing chain relaxation, and the core region being isotropic. This core-shell structure is used to explain the increase in elastic modulus with decreasing PVA nanofiber diameter as the shell component remained fairly constant at around $30 \mathrm{~nm}$, meaning that the relative contribution of the shell region increases with decreasing fiber diameter. Properties of these PVA fibers indicated an increase in elastic modulus, as measured by single fiber AFM bending tests, for diameters below $300 \mathrm{~nm}$. Elastic moduli up to $13 \mathrm{GPa}$, six times that of bulk PVA, were reported for fibers with diameters just below $100 \mathrm{~nm}$. Although these values are superior to most reported data for electrospun fibers these values still do not rival solid-state drawn solution-spun PVA fibers possessing Young's moduli up to $70 \mathrm{GPa}$ and tensile strengths of approximately $2 \mathrm{GPa}$ [86].

Arinstein et al. [87] related the size of oriented regions in electrospun nylon 6,6 nanofibers to the rapid increase in Young's modulus and quantified the percolation of the cross-section area required by using a modified Onsager model. A value of about $300 \mathrm{~nm}$ for a critical fiber diameter was found, below which a rapid increase in Young's modulus is initiated. Arinstein et al. [88] also reported an increase in Young's modulus in electrospun nylon 6,6 nanofibers with decreasing fiber diameter (Figure 13). Here the authors suggest that the rapid increase in Young's modulus with decreasing fiber diameter is not the result of the small increase in observed crystallinity or orientation of the crystallites. Instead they ascribed the increase in mechanical properties to orientation of amorphous regions when their sizes are comparable or smaller than the nanofiber diameter.

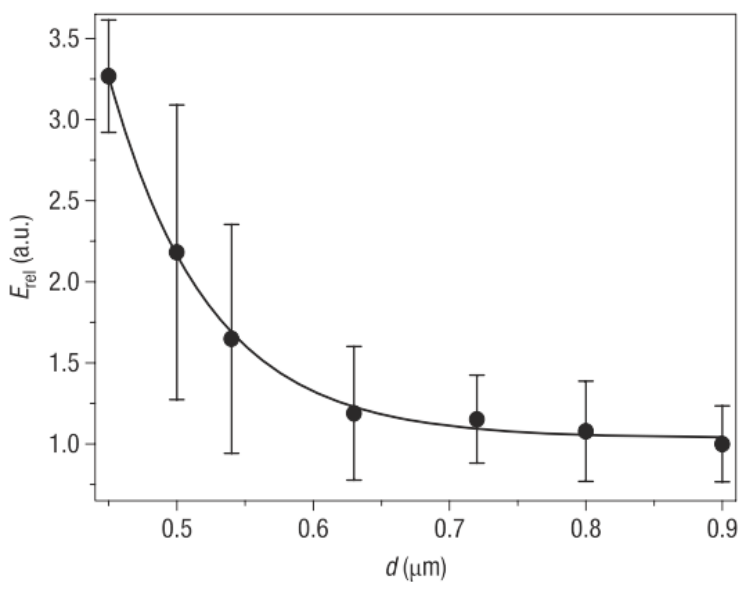

Figure 13. Relative Young's modulus $E_{\text {rel }}\left(E_{r e l}=E / E_{b u l k}\right)$ of electrospun PA 6,6 nanofibers as a function of their diameters [88]. Reproduced with permission from Arinstein et al., 2007. Copyright Nature Publisher. 
Most studies show similar to results reported by Arinstein et al. [86], i.e., a three-fold increase in Young's modulus of thin electrospun fibers compared to that of bulk polymer. Naraghi et al. reported an increase in Young's modulus for electrospun PAN fibers smaller than $300 \mathrm{~nm}$, with a three-fold increase in modulus for diameters ranging from 100-200 nm [89]. Pai et al. reported a similar increase in Young's modulus of individual electrospun fibers of poly(trimethyl hexamethylene terephthalamide) (PA 6(3)T) [80]. Here, the Young's modulus was found to increase for fibers with diameters smaller than $500 \mathrm{~nm}$, with a maximum modulus value reported of around $6 \mathrm{GPa}$ for $170 \mathrm{~nm}$ fibers, i.e., three times the bulk modulus.

One recent example of a more significant property improvement in electrospun fibers that was ascribed to confinement effects was reported for polyacrylonitrile (PAN). Young's moduli up to $48 \mathrm{GPa}$ and tensile strengths up to $1.75 \mathrm{GPa}$ were reported by Dzenis and coworkers [90] for individual PAN nanofibers, approaching typical values for high performance fibers. Unlike traditional high performance fibers, where an increase in mechanical properties is often accompanied with a sacrifice in strain at break, these ultrafine PAN nanofibers were also simultaneously stiff and ductile. The highest value of toughness was achieved from the thinnest electrospun nanofiber with a diameter of $138 \mathrm{~nm}$. Similar to Arinstein et al. their increase in mechanical properties was not ascribed to increased crystallinity (Figure 14) as crystallization was restrained by the fast solidification that resulted from the rapid evaporation of solvent. Again it was proposed that the increase in mechanical properties was mainly related to confined molecular orientation of amorphous regions with decreasing nanofiber diameter.

The mechanical properties of these electrospun PAN fibers are particularly impressive, with the reported Young's modulus of $48 \mathrm{GPa}$ approaching or even exceeding theoretical values for atactic PAN (55 GPa) [91] or isotactic PAN having a 3/1 helical conformation (35 GPa) [91], although theoretical calculations have indicated that the crystal modulus of PAN taking a planar-zigzag conformation could reach values as high as $130 \mathrm{GPa}$ [92]. In contrast to most other electrospun fibers, including PAN, where confinement effects typically result in a moderate increase in modulus $[80,85,87,88]$, here a nanofiber modulus is reported that is $\sim 25$ times greater than that of bulk PAN. This is in stark contrast to studies of Naraghi et al. [89], who reported only a three-fold increase in Young's modulus for electrospun PAN fibers with a maximum value of $7 \mathrm{GPa}$ for a $150 \mathrm{~nm}$ fiber. Similarly, these high performance PAN nanofibers also outperform most conventional wet-spun high strength acrylic fibers with typical Young's moduli of 10-20 GPa and tensile strengths of 1-1.5 GPa [93]. In fact, the values reported for these electrospun PAN fibers exceed those of super-drawn solution-spun ultra-high molecular weight PAN fibers by Kanamoto and co-workers who obtained tensile moduli of $35 \mathrm{GPa}$ and strengths 
of 1.8 GPa for fibers of draw-ratio 80, exhibiting extremely high levels of chain orientation [92].
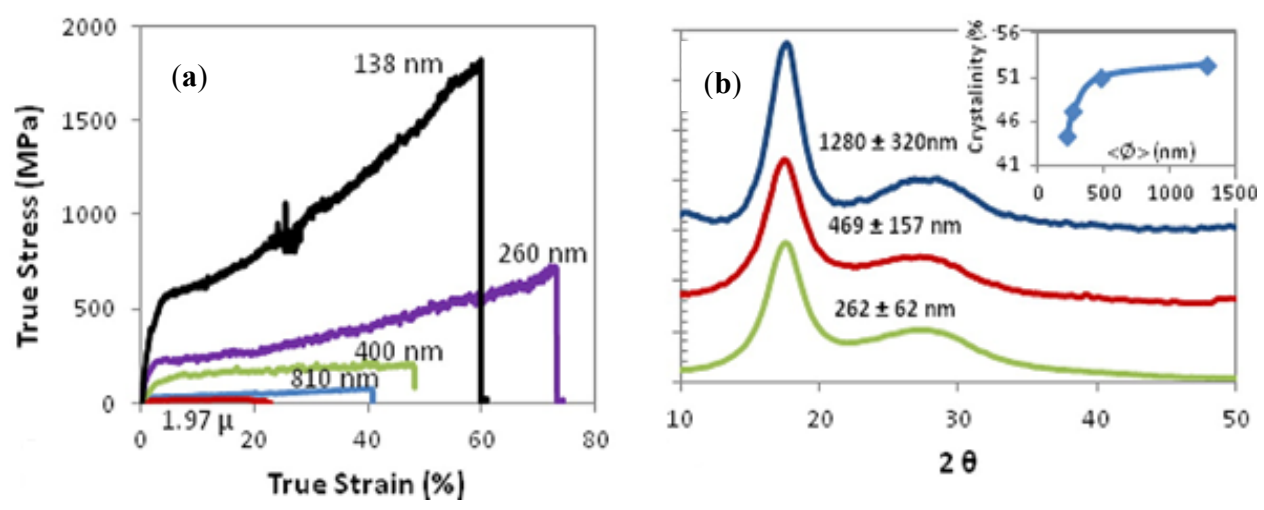

Figure 14. (a) Stress-strain curves of electrospun PAN nanofibers with different diameters (b) XRD patterns of nanofiber bundles with various fibre diameters and corresponding degree of crystallinity (inset) [90]. Reproduced with permission from Papkov, D. et al., 2013, Copyright American Chemistry Society.

The underlying mechanisms of these exceptional properties are therefore still to be debated. The high modulus of the PAN nanofibers seems not related to their crystallinity as the reported crystallinity $(<50 \%)$ does not significantly increase with decreasing fiber diameter and is also less than that of conventional PAN fiber $(\sim 65 \%)$ [94]. Since flow-induced orientation is difficult to achieve during fiber spinning due to fast chain relaxation, and because these fibers are not post-drawn, it is difficult to envisage higher levels of chain extension in these as-spun nanofibres than in the super-drawn UHMW-PAN fibers by Sawai et al. [92]. Moreover, as the estimated coil size of a PAN molecule with a molar mass of 150,000 is of the order of $30-40 \mathrm{~nm}$ based on its mean square end-to-end distance $\left.\left(<R^{2}\right\rangle=C_{\infty} n l^{2}\right)[95,96]$ it is also difficult to imagine the extreme confinement effects in $138 \mathrm{~nm}$ fibers which are necessary to explain such property improvement. Nevertheless, the reported mechanical properties of these electrospun PAN fibers are very interesting and require further studies.

In summary, the relatively poor mechanical properties of flexible chain based polymer nanofibers are mainly ascribed to two phenomena. The first one is related to the relatively low degree of chain orientation in these systems. Orientation of macromolecules can be usually introduced from a random coil morphology when the product of the polymer chain relaxation time and the strain rate in the electrospinning process is greater than 0.5 [57]. Given that strain rates can be up to $10^{5} \mathrm{~s}^{-1}$ while viscoelastic relaxation times are found to be tens to hundreds of 
milliseconds $[97,98]$, some level of chain orientation can be potentially generated. However, residual solvents in the deposited nanofibers can accelerate chain relaxation and lead to shorter relaxation times [57]. Hence, relaxation of polymer chains could be suppressed by rapid solvent evaporation and fiber solidification [58]. As relaxation times of flexible chain polymers are typically very short, orientation induced in the initial spinning process may rapidly disappear before solidification. It is for this reason that conventional high performance fibers based on flexible chain polymers are post-drawn in the solid state below the melting temperature as this will prevent chain relaxation after orientation. Similarly, low degrees of molecular orientation are to be expected in electrospun fibers based on flexible chain polymers, explaining their poor mechanical properties. Nonetheless, the mechanics in achieving chain orientation in electrospun fibers is still controversial due to the lack of systematically studies. For instant, most of characterizations on molecular orientation and mechanical properties in electrospun nanofibers are conducted on nanofiber mats or bundles rather than single fibers [99]. Therefore, in future research more attentions should be paid to single fiber characterization in order to establish better structure-processing-property relationships.

The second reason for the relatively poor mechanical properties of electrospun fibers is that full chain extension is nearly impossible to realize during the electrospinning process in the case of flexible chain polymers, which is crucial for obtaining a high performance fiber with properties approaching the theoretical crystal moduli listed in Table 1. With the exception Dzenis and co-workers' PAN nanofiber [90], the differences between experimentally reported Young's moduli of electrospun nanofibers and these ultimate values are very large. Assuming the hypothetical case that polymer chain extension from random coil morphology is induced by virtue of reducing the nanofiber diameter to the same level as the size of crystallites or even the size of a single chain one might envisage a high modulus. Respective average crystallite size and chain size of PA 6,6 are around $4 \mathrm{~nm}$ and $0.1 \mathrm{~nm}$ [87], respectively, and polymer chains could be forced to extent and orient along the fiber axis if fiber diameters would go down to these levels. In such a case the Young's modulus of the fiber would be potentially approaching near theoretical values. However, even in such a hypothetical case it is expected that such ultra-thin fibers would be extremely weak as there would be insufficient overlap between chains, resulting in poor stress transfer.

\subsection{Electrospun Nanofibers Based on Rigid Chain Polymers}

\subsubsection{Electrospun PPTA Fibers}

While chain extension in the case of flexible chain polymers can effectively be only achieved by solid-state drawing, the extension of polymer chains can be readily 
build in by polymer scientists when using rigid rod polymers. Rigid rod polymers have a significant advantage over flexible chain polymers as these systems can be oriented during the spinning process without the need of a post-drawing process to induce chain extension.

Inspired by the success of high performance aramid fibers the electrospinning of poly(p-phenylene terehthalamide (PPTA) and characterization of the obtained nanofiber structures was carried out by Srinivasan and Reneker [100].

In their studies a homogeneous isotropic solution was formed by dissolving PPTA fibers (Kevlar $49^{\circledR}$ ) at a concentration of $2-3 \mathrm{wt} \%$ in a solvent of $95 \%-98 \%$ sulphuric acid. A water bath was used for extracting the solvent and collecting the electrospun nanofibers $12-18 \mathrm{kV}$ high voltages were applied between two electrodes at a distance of $3 \mathrm{~cm}$. Nanofibers with diameters ranging from $40 \mathrm{~nm}$ to hundreds of nanometers were produced (Figure 15). The meridional and equatorial reflection of the as-spun and at $400{ }^{\circ} \mathrm{C}$ annealed PPTA nanofibers were shown in dark field mode transmission electron microscopy (TEM) and revealed some order in the fibers. However, only moderate chain orientation in these annealed PPTA nanofibers was demonstrated using electron diffraction. Moreover, a continuous electrospinning process and mechanical properties of the deposited nanofibers were not reported.

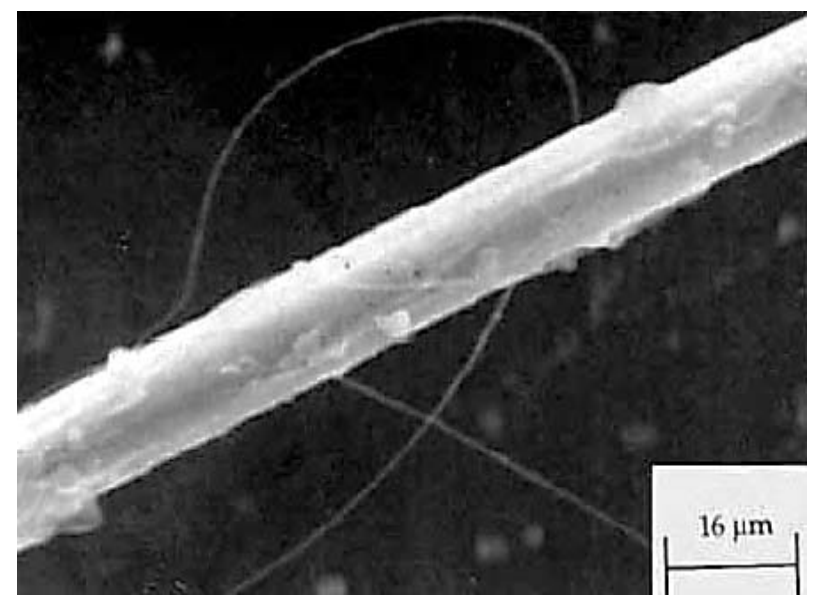

Figure 15. A single electrospun PPTA fiber together with a single $\operatorname{Kevlar}^{\circledR} 49$ fiber [100]. Reproduced with permission from Srinivasan \& Reneker, 1995. Copyright John Wiley \& Sons.

Traditional high performance $p$-aramid fibers are typically produced from anisotropic PPTA solutions with concentrations of around 19-20 wt\% as shown in Figure 16 [94]. However, in the work of Srinivasan and Reneker, spinning solutions employed (2-3wt\% ) were in their isotropic phase, which is outside the regime 
needed for flow induced molecular orientation as in the case of dry-jet wet spinning of high performance $p$-aramid fibers. Nevertheless, electrospinning of rigid rod polymers like aramids could still show promise once anisotropic PPTA solutions are used under optimized conditions, as it avoids problems related to chain relaxation in as-spun fibers.

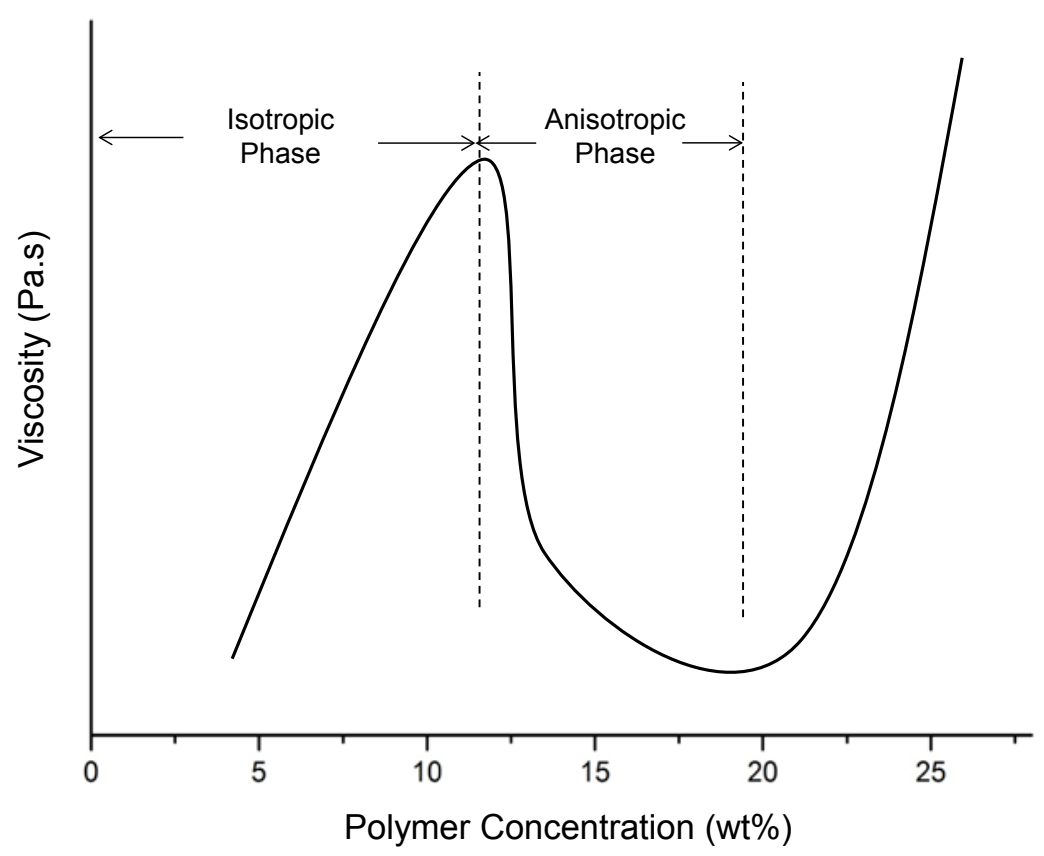

Figure 16. Liquid crystalline behavior of PPTA solution, indicating an isotropic phase at concentrations below $12 \mathrm{wt} \%$ and an anisotropic phase between concentrations of $12 \mathrm{wt} \%$ to $20 \mathrm{wt} \%$.

\subsubsection{Electrospun Polyimide Nanofiber}

Poly(p-phenylene biphenyltetracarboxamide) (BPDA/PDA) is a high performance aromatic polyimide with great mechanical properties but a less rigid structure compared to PPTA [75] (Figure 17). Because of the rigid macromolecular backbone, it is insoluble in common organic solvents and thus not directly spinnable from solutions.

However, the precursor of BPDA/PDA, poly( $p$-phenylene biphenyltetracarboxamide acid) (BP-PAA) (Figure 18) shows good solubility in common organic solvents and it is therefore feasible to electrospin BP-PAA precursor fibers and subsequently convert them into BPDA-PDA polyimide nanofibers through a heat treatment.

Hou and coworkers $[101,102]$ investigated the electrospinning process and mechanical properties of these polyimide nanofibers. Under proper spinning 
condition, a well-aligned BP-PAA nanofiber mat was obtained on a high-speed collector and the aligned polyamic acid nanofiber samples were imidized into polyimide nanofibers. During this imidization process, the rigid-rod molecular chains tend to become oriented and extended along the fiber axis (Figure 18).

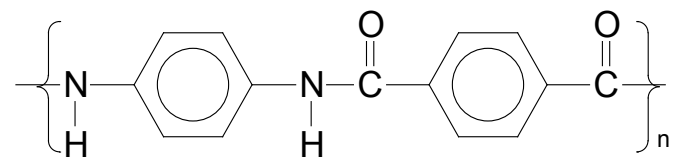<smiles>Cc1ccc(N2C(=O)c3ccc(-c4ccc5c(c4)C(O)N(C)C5=O)cc3C2=O)cc1</smiles>

Figure 17. Structural formulas of (a) PPTA and (b) BPDA/PDA polyimide.

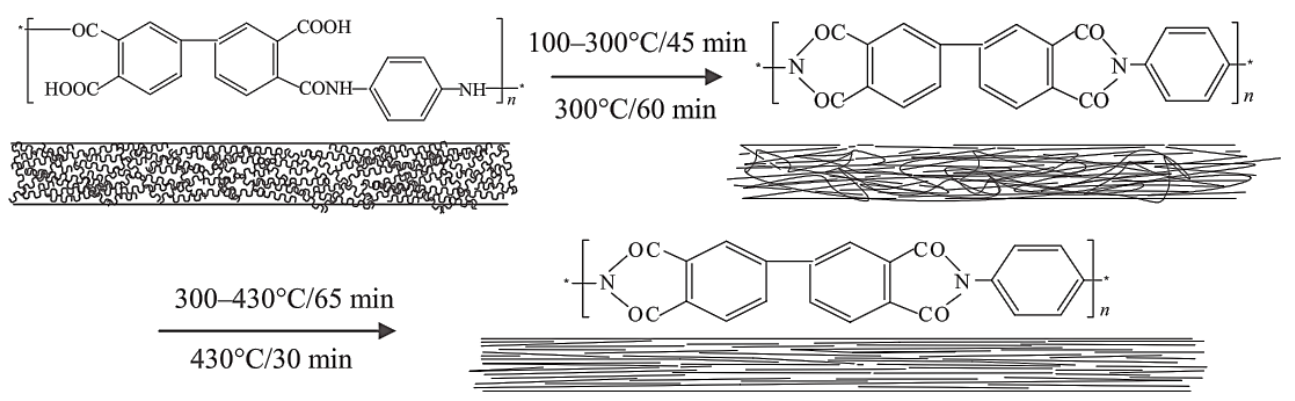

Figure 18. A schematic diagram of the imidization process [75]. Reproduced with permission from Huang et al., 2006. Copyright John Wiley \& Sons.

Mechanical tests indicated that the average tensile strength and modulus of these nanofiber mats with fiber diameters of around $300 \mathrm{~nm}$ were $660 \mathrm{MPa}$ and $15 \mathrm{GPa}$, respectively. Further studies showed that the ultimate tensile strength and Young's modulus could reach values of $1.7 \mathrm{GPa}$ and $76 \mathrm{GPa}$, which are comparable to conventional BPDA-PDA fibers [103] and are in the region of commercial high performance fibers. 


\subsection{Other Routes to High Performance Nanofibers}

\subsubsection{CNT Reinforced Polymer Nanofibers}

Carbon nanotubes, due to their excellent mechanical properties, have been regarded as ideal reinforcement candidates for nanocomposites [104-106]. Basically, the Young's modulus and tensile strength of single-wall carbon nanotubes (SWCNTs) are reported to reach about $1 \mathrm{TPa}$ and tens of GPa's [107,108], respectively, outperforming most other materials.

Generally, CNTs are however prone to aggregation and bundling because of their small diameter and strong Van der Waals interactions, lowering their reinforcing capability. Therefore, three main challenges are encountered when using carbon nanotubes as nano-reinforcements in polymer fibers. Firstly, the creation of a homogenous spinnable dispersion of CNTs. Secondly, a good interfacial adhesion and stress transfer, and finally orientation of the CNTs in the polymer fiber [109-111]. Electrospinning is a promising technique to produce CNT reinforced nanofibers since it has the potential to debundle and align CNTs along the fiber axis and thus effectively reinforce the nanofiber [112-114], while interfacial interactions between CNTs and host polymer can be enhanced by functionalization of the CNTs [115].

Sen et al [115] reported on ester-functionalized SWCNTs reinforced electrospun polyurethane (PU) nanofibers. Mechanical tests showed that the tensile strength of these ester-functionalized SWCNT reinforced nanofiber membranes were improved by $104 \%$, while elastic moduli were increased by $250 \%$ compared to pure PU membranes, with improvements of these properties being mainly attributed to alignments and improved interfacial interactions between SWCNTs and polymer matrix.

Baji et al. investigated the mechanical properties of multi-wall carbon nanotube (MWCNT) reinforced nylon 6,6 nanofibers [116]. In their study, carboxylic acid functional groups modified MWCNTs were mixed together with nylon 6,6 in different concentrations. These mixtures were electrospun into aligned nanofibers using parallel plate electrodes. Mechanical tests showed that the tensile strength and Young's modulus of the composite nanofibers increased with CNT loading, reaching peak values (from about $0.32 \mathrm{GPa}$ and 1.2 GPa to $0.65 \mathrm{GPa}$ and $3.5 \mathrm{GPa}$, respectively) for CNT concentrations of $7.5 \mathrm{wt} \%$. This significant improvement in mechanical properties was claimed to be due to among others the good dispersion and alignment of the MWCNTs along the nanofiber axis as confirmed by TEM. Other studies involving CNT reinforced nanofibers were reported by Hou et al. [117], Jose et al. [118], Lu et al. [119], and Wang et al. [120].

A careful analysis of the reinforcing efficiency of CNTs in the composite fibers should be emphasized here since high reinforcing efficiency of CNTs in composites is generally a good indication of effective dispersion, interfacial interaction and 
alignment. Wang et al. [121] reported on solid-state drawn nanocomposite PVA/SWCNTs tapes and found a remarkable reinforcing efficiency for systems incorporating up to $1 \mathrm{wt} \%$ of SWCNTs. Further studies on nanotube reinforced electrospun PVA nanofibers (Figure 19) revealed similar high reinforcing efficiencies, with a back-calculated SWCNT Young's modulus of around 0.85 TPa [120], i.e., close to its theoretical $1 \mathrm{TPa}$ value.

Despite various developments in CNT reinforced nanofibers, in many cases, eliminating agglomeration and achieving homogenous spinnable solutions and good alignment remain a challenge especially at higher CNT concentrations. Moreover, even for systems that report significant property improvements these properties are often still not very impressive when compared to commercial high performance fibers.

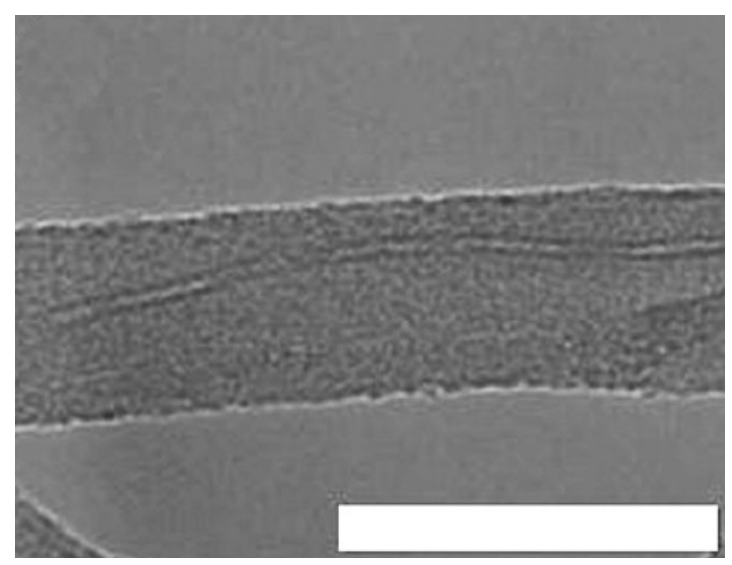

Figure 19. TEM micrograph of an individual MWCNT reinforced electrospun PVA nanofiber, showing an aligned MWCNT in a polymer nanofiber (scale bar $100 \mathrm{~nm})[120]$.

\subsubsection{Electrospun Polymer-derived Carbon Nanofibers}

Another interesting approach towards high strength and high modulus nanofibers is the development of carbon nanofibers. Polymer nanofiber precursors prepared via electrospinning followed by carbonization have been investigated [122]. Several polymers have been investigated as precursors for carbon fibers. Principally, polyacrylonitrile is used [123-127], while pitches [128,129], poly(vinyl alcohol) (PVA) [130], polyimide (PI) [131], poly(vinylidene fluoride) (PVDF) [132], and polybenzimidazol (PBI) [133] have also been utilized.

Zussman et al. created single electrospun PAN-derived carbon nanofibers with different fiber diameters possessing Young's moduli of $63 \pm 7$ GP and tensile 
strengths between $0.32 \mathrm{GPa}$ and $0.9 \mathrm{GPa}$. Inferior mechanical properties of these electrospun PAN-based carbon nanofibers compared to commercial carbon fibers resulted from the relatively poor molecular orientation and disarrangements in the core-shell structure of the carbon nanofibers [124]. PAN-based carbon nanofibers were also prepared by Zhou et al. [127]. Young's moduli of 40 to $60 \mathrm{GPa}$ and tensile strengths of 0.3 to $0.6 \mathrm{GPa}$ for carbon nanofiber bundles were achieved and mechanical properties were found to increase with carbonization temperature. In addition, the authors proposed possible ways to further enhance the mechanical properties of these PAN-based carbon nanofibers, i.e., by post-drawing of precursor fibers; the stabilization and carbonization of nanofibers under tension; and the use of PAN copolymer as a precursor. Optimized process conditions for strong carbon nanofibers based on PAN were reported by Chasiotis and co-workers [134]. Both the PAN precursor nanofibers and carbon nanofibers were smooth and uniform (Figure 20). Young's moduli of $191 \pm 58 \mathrm{GPa}$ upon carbonization at $1700{ }^{\circ} \mathrm{C}$ and tensile strengths of $3.52 \pm 0.64 \mathrm{GPa}$ upon carbonization at $1400^{\circ} \mathrm{C}$ were reported, and were attributed to an increase in crystallites in the carbon nanofibers, making these fibers approach the mechanical performance of standard high-strength carbon fibers.

(a)

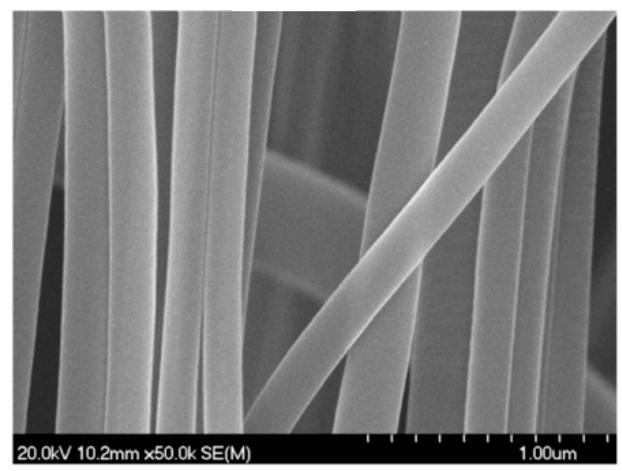

(b)

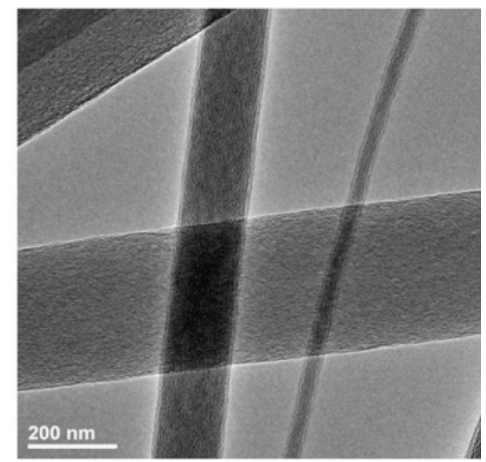

Figure 20. (a) SEM micrograph and (b) TEM micrograph showing homogenous morphology of carbon nanofibers [134]. Reproduced with permission from Arshad et al., 2011. Copyright by Elsevier Publisher. 


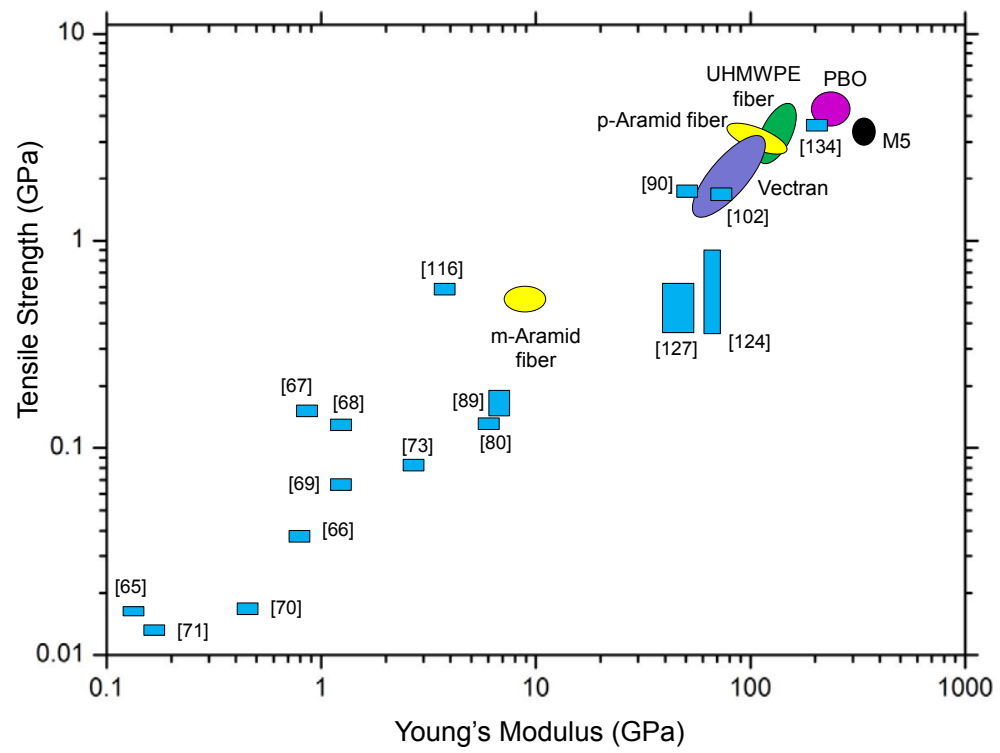

Figure 21. Mechanical properties of traditional high performance fibers and electrospun nanofibers with respect to Young's modulus and tensile strength. Commercial high-performance fibers show typical tensile strengths of 3-4 GPa and moduli of around 100-300 GPa, while most electrospun fibers typically possess tensile strengths $<0.3 \mathrm{GPa}$ and Young's moduli $<3 \mathrm{GPa}[65-71,73]$. Some high performance electrospun nanofibers have been reported based on polyimide [102], polyacrylonitrile [90] and carbon nanofibers from electrospun PAN precursors [134].

\section{Conclusions}

Electrospinning has proven to be an efficient method to produce thin fibers with diameters down to the nano-scale. However, the mechanical properties of these nanofibers are often well below those of fibers made by conventional processes such melt- or solution spinning (see Figure 21). The main reason for this being the competition between flow-induced chain orientation and chain relaxation before fiber solidification, leading to low degrees of molecular orientation in as-spun fibers. In conventional polymer fiber processing, chain alignment is induced by drawing the as-spun fiber in the solid-state below the melting temperature into a highly oriented structure as here relaxation times are infinite. In order to achieve similar high levels of chain orientation and chain extension in nanofibers based on flexible chain polymers it is vital to apply a post-stretching step.

Although some evidence exists of confinement induced molecular orientation in the case of ultra-fine nanofibers, the orientation and particularly chain extension achieved is often rather limited, leading to only moderate improvements in 
Young's modulus (typically 2-4 times bulk polymer), well below those attainable in commercial melt- or solution spun fibers (typically 10-100 times bulk polymer).

As the introduction of a post-drawing step in commercial electrospinning processes may prove technologically challenging, the use of rigid-rod polymers as an alternative to flexible chain polymers may be more promising as here chains have already build in chain extension and can be readily oriented during spinning. Other alternative routes worth pursuing are the use of nano-reinforcements such as carbon nanotubes or transforming polymer precursor fibers into carbon nanofibers, with especially the latter showing some great promise for future work.

Acknowledgments: The first author gratefully acknowledges the support from the China Scholarship Council (CSC).

Author Contributions: All authors contributed equally to this work.

Conflicts of Interest: The authors declare no conflict of interest.

\section{References}

1. Doshi, J.; Reneker, D.H. Electrospinning process and applications of electrospun fibers. J. Electrost. 1995, 35, 151-160.

2. Reneker, D.H.; Chun, I. Nanometre diameter fibres of polymer, produced by electrospinning. Nanotechnology 1996, 7, 216.

3. Fong, H.; Chun, I.; Reneker, D. Beaded nanofibers formed during electrospinning. Polymer 1999, 40, 4585-4592.

4. Kim, J.S.; Reneker, D.H. Mechanical properties of composites using ultrafine electrospun fibers. Polym. Compos. 1999, 20, 124-131.

5. Jaeger, R.; Bergshoef, M.M.; Batlle, C.M.I.; Schönherr, H.; Julius Vancso, G. Electrospinning of Ultra-thin Polymer Fibers, Macromolecular Symposia, 1998; Wiley Online Library: Hoboken, NJ, USA, 1998; pp. 141-150.

6. Bognitzki, M.; Czado, W.; Frese, T.; Schaper, A.; Hellwig, M.; Steinhart, M.; Greiner, A.; Wendorff, J.H. Nanostructured fibers via electrospinning. Adv. Mater. 2001, 13, 70-72.

7. Fang, X.; Reneker, D. DNA fibers by electrospinning. J. Macromol. Sci. Part B Phys. 1997, 36, 169-173.

8. Fong, H.; Reneker, D.H. Elastomeric nanofibers of styrene-butadiene-styrene triblock copolymer. J. Macromol. Sci. Part B Phys. 1999, 37, 3488-3493.

9. Ramakrishna, S. An Introduction to Electrospinning and Nanofibers; World Scientific Publishing Co. Pte. Ltd: Singapore, 2005; pp. 90-154.

10. Xu, C.; Inai, R.; Kotaki, M.; Ramakrishna, S. Aligned biodegradable nanofibrous structure: A potential scaffold for blood vessel engineering. Biomaterials 2004, 25, 877-886.

11. Huang, Z.-M.; Zhang, Y.-Z.; Kotaki, M.; Ramakrishna, S. A review on polymer nanofibers by electrospinning and their applications in nanocomposites. Compos. Sci. Technol. 2003, 63, 2223-2253. 
12. Greiner, A.; Wendorff, J. Functional self-assembled nanofibers by electrospinning. In Self-Assembled Nanomaterials I; Springer Berlin Heidelberg: Berlin, Germany, 2008; pp. 107-171.

13. Wang, J.; Kim, S.C.; Pui, D.Y. Investigation of the figure of merit for filters with a single nanofiber layer on a substrate. J. Aerosol. Sci. 2008, 39, 323-334.

14. Nair, L.S.; Bhattacharyya, S.; Bender, J.D.; Greish, Y.E.; Brown, P.W.; Allcock, H.R.; Laurencin, C.T. Fabrication and optimization of methylphenoxy substituted polyphosphazene nanofibers for biomedical applications. Biomacromolecules 2004, 5, 2212-2220.

15. Lee, K.; Kim, H.; Khil, M.; Ra, Y.; Lee, D. Characterization of nano-structured poly ( $\varepsilon$-caprolactone) nonwoven mats via electrospinning. Polymer 2003, 44, 1287-1294.

16. Lee, K.H.; Kim, H.Y.; Ryu, Y.J.; Kim, K.W.; Choi, S.W. Mechanical behavior of electrospun fiber mats of poly (vinyl chloride)/polyurethane polyblends. J. Polym. Sci. Part B Polym. Phys. 2003, 41, 1256-1262.

17. Huang, Z.-M.; Zhang, Y.; Ramakrishna, S.; Lim, C. Electrospinning and mechanical characterization of gelatin nanofibers. Polymer 2004, 45, 5361-5368.

18. Hansen, L.M.; Smith, D.J.; Reneker, D.H.; Kataphinan, W. Water absorption and mechanical properties of electrospun structured hydrogels. J. Appl. Polym. Sci. 2005, 95, 427-434.

19. Matthews, J.A.; Wnek, G.E.; Simpson, D.G.; Bowlin, G.L. Electrospinning of collagen nanofibers. Biomacromolecules 2002, 3, 232-238.

20. Staudinger, H. Über polymerisation. Ber. Deutsch. Chem. Ges. (A and B Series) 1920, 53, 1073-1085. (in German).

21. Heeger, A.J. Nobel Lecture: Semiconducting and metallic polymers: The fourth generation of polymeric materials. Rev. Mod. Phys. 2001, 73, 681-700.

22. Dasgupta, S.; Hammond, W.B.; Goddard, W.A. Crystal structures and properties of nylon polymers from theory. J. Am.Chem. Soc. 1996, 118, 12291-12301.

23. Cook, J.G. Handbook of Textile Fibres: Man-Made Fibres; Woodhead Publishing Limited: Cambridge, UK, 1984; pp. 192-391.

24. Staudinger, H. Die Hochmolekularen im festen Zustand. In Die Hochmolekularen Organischen Verbindungen-Kautschuk und Cellulose; Springer: Berlin/Heidelberg, Germany, 1932; pp. 105-123, (in German).

25. Meyer, K.H.; Lotmar, W.; Pankow, G. Sur le chlorure de poly-phosphornitrile, caoutchouc inorganique. Helvetica. Chimica. Acta. 1936, 19, 930-948. (in French).

26. Treloar, L.G. Calculations of elastic moduli of polymer crystals: I. Polyethylene and nylon 66. Polymer 1960, 1, 95-103.

27. Peijs, A.; Jacobs, M.; Lemstra, P. High-performance polyethylene fibers. In Comprehensive Composite Materials. Vol. 1. Fiber Reinforcements and General Theory of Composites; Chou, T.W., Kelly, A., Zweben, C., Eds.; Elsevier: Amsterdam, the Netherlands, 2000; pp. 263-301.

28. Hageman, J.; Meier, R.J.; Heinemann, M.; De Groot, R. Young modulus of crystalline polyethylene from ab initio molecular dynamics. Macromolecules 1997, 30, 5953-5957. 
29. Crist, B. The ultimate strength and stiffness of polymers. Ann. Rev. Mater. Sci. 1995, 25, 295-323.

30. Manley, T.; Martin, C. Elastic modulus of linear polymer crystals. Polymer 1973, 14, 491-496.

31. Nakamae, K.; Nishino, T. Crystal moduli of high polymers and their temperature dependence. In Integration of Fundamental Polymer Science and Technology-5; Lemstra, P., Kleintjens, L., Eds.; Springer: Houten, the Netherlands, 1991; pp. 121-130.

32. Lemstra, P.; Kirschbaum, R.; Ohta, T.; Yasuda, H. High-strength/high-modulus structures based on flexible macromolecules: Gel-spinning and related processes. In Developments in Oriented Polymers-2; Springer: Houten, the Netherlands, 1987; pp. 39-77.

33. Lemstra, P.; Van Aerle, N.; Bastiaansen, C. Chain-extended polyethylene. Polym. J. 1987, 19, 85-98.

34. Lemstra, P.; Bastiaansen, C.; Meijer, H. Chain-extended flexible polymers. Die Angew. Makromol. Chem. 1986, 145, 343-358.

35. Bastiaansen, C.W.; Simmelink, J.A.P.M. Solution of ultra-high molecular weight polyethylene. US Patent 5,428,079. filed 30 July 1991, and issued 27 June 1995.

36. Capaccio, G.; Ward, I. Ultra-high-modulus linear polyethylene through controlled molecular weight and drawing. Polym. Eng. Sci. 1975, 15, 219-224.

37. Cansfield, D.; Capaccio, G.; Ward, I. The preparation of ultra-high modulus polypropylene films and fibres. Polym. Eng. Sci. 1976, 16, 721-724.

38. Zwijnenburg, A.; Pennings, A. Longitudinal growth of polymer crystals from flowing solutions III. Polyethylene crystals in Couette flow. Colloid Polym. Sci. 1976, 254, 868-881.

39. Zwijnenburg, A.; Pennings, A. Longitudinal growth of polymer crystals from flowing solutions. IV. The mechanical properties of fibrillar polyethylene crystals. J. Polym. Sci. Polym. Lett. Ed. 1976, 14, 339-346.

40. Ajji, A.; Coates, P.; Dumoulin, M.; Ward, I. Solid Phase Processing of Polymers; Carl Hanser Verlag: Munich, Germany, 2000; pp. 85-210.

41. Smith, P.; Lemstra, P.J. Ultrahigh-strength polyethylene filaments by solution spinning/drawing, 2. Influence of solvent on the drawability. Die Makromol. Chem. 1979, 180, 2983-2986.

42. Smith, P.; Lemstra, P.J.; Pijpers, J.P. Tensile strength of highly oriented polyethylene. II. Effect of molecular weight distribution. J. Polym. Sci.: Polym. Phys. Ed. 1982, 20, 2229-2241.

43. Smith, P.; Lemstra, P.; Pijpers, J.; Kiel, A. Ultra-drawing of high molecular weight polyethylene cast from solution. Colloid Polym. Sci. 1981, 259, 1070-1080.

44. Smith, P.; Lemstra, P.J. Filaments of high tensile strength and modulus. US Patent 4,430,383. filed 30 September 1982, and issued 7 February 1984.

45. Kwolek, S.L. Optically anisotropic aromatic polyamide dopes. US Patent 3,671,542. filed 23 May 1969, and issued 20 June 1972.

46. Kwolek, S.; Morgan, P.; Schaefgen, J.; Gulrich, L. Synthesis, anisotropic solutions, and fibers of poly (1, 4-benzamid). Macromolecules 1977, 10, 1390-1396.

47. Yang, H. Kevlar Aramid Fiber; John Wiley \& Sons: Chichester, NH, USA, 1993; pp. 1-22. 
48. Bair, T.; Morgan, P.; Killian, F. Poly (1, 4-phenyleneterephthalamides). polymerization and novel liquid-crystalline solutions. Macromolecules 1977, 10, 1396-1400.

49. Blades, H. High modulus, high tenacity poly (p-phenylene terephthalamide) fiber. US Patent 3,869,430. filed 30 June 1972, and issued 4 March 1975.

50. Dobb, M.; Johnson, D.; Saville, B. Supramolecular structure of a high-modulus polyaromatic fiber (Kevlar 49). J. Polym. Sci.: Polym. Phys. Ed. 1977, 15, 2201-2211.

51. Hearle, J.W. High-Performance Fibres; Woodhead Publishing: Cambridge, UK, 2001; Volume 15, pp. 93-155.

52. Kumar, S.; Dang, T.D.; Arnold, F.E.; Bhattacharyya, A.R.; Min, B.G.; Zhang, X.; Vaia, R.A.; Park, C.; Adams, W.W.; Hauge, R.H. Synthesis, Structure, and Properties of PBO/SWNT Composites. Macromolecules 2002, 35, 9039-9043.

53. Kitagawa, T.; Murase, H.; Yabuki, K. Morphological study on poly(pphenylenebenzobisoxazole) (PBO) fiber. J. Polym. Sci. Part B Polym. Phys. 1998, 36, 39-48.

54. Choe, E.W.; Kim, S.N. Synthesis, spinning, and fiber mechanical properties of poly (p-phenylenebenzobisoxazole). Macromolecules 1981, 14, 920-924.

55. Sikkema, D.J. Design, synthesis and properties of a novel rigid rod polymer, PIPD or M5: High modulus and tenacity fibres with substantial compressive strength. Polymer 1998, 39, 5981-5986.

56. Sirichaisit, J.; Young, R. Tensile and compressive deformation of polypyridobisimidazole (PIPD)-based M5 rigid-rod polymer fibres. Polymer 1999, 40, 3421-3431.

57. Greiner, A.; Wendorff, J.H. Electrospinning: a fascinating method for the preparation of ultrathin fibers. Angew. Chem. Int. Ed. 2007, 46, 5670-5703.

58. Li, D.; Xia, Y. Electrospinning of nanofibers: reinventing the wheel? Adv. Mater. 2004, 16, 1151-1170.

59. Petrik, S.; Maly, M. Production Nozzle-less Electrospinning Nanofiber Technology, MRS Proceedings, 2009; Materials Research Society: Warrendale, PA, USA, 2009.

60. Erisken, C.; Kalyon, D.M.; Wang, H. A hybrid twin screw extrusion/electrospinning method to process nanoparticle-incorporated electrospun nanofibres. Nanotechnology 2008, 19, 165302.

61. Senturk-Ozer, S.; Ward, D.; Gevgilili, H.; Kalyon, D.M. Dynamics of electrospinning of poly (caprolactone) via a multi-nozzle spinneret connected to a twin screw extruder and properties of electrospun fibers. Polym. Eng. Sci. 2013, 53, 1463-1474.

62. Badrossamay, M.R.; McIlwee, H.A.; Goss, J.A.; Parker, K.K. Nanofiber assembly by rotary jet-spinning. Nano Lett. 2010, 10, 2257-2261.

63. Rein, D.M.; Shavit-Hadar, L.; Khalfin, R.; Cohen, Y.; Shuster, K.; Zussman, E. Electrospinning of ultrahigh-molecular-weight polyethylene nanofibers. J. Polym. Sci. Part B Polym. Phys. 2007, 45, 766-773.

64. Peijs, T.; Rijsdijk, H.; De Kok, J.; Lemstra, P. The role of interface and fibre anisotropy in controlling the performance of polyethylene-fibre-reinforced composites. Compos. Sci. Technol. 1994, 52, 449-466. 
65. Carrizales, C.; Pelfrey, S.; Rincon, R.; Eubanks, T.M.; Kuang, A.; McClure, M.J.; Bowlin, G.L.; Macossay, J. Thermal and mechanical properties of electrospun PMMA, PVC, Nylon 6, and Nylon 6, 6. Polym. Adv. Technol. 2008, 19, 124-130.

66. Bazbouz, M.B.; Stylios, G.K. The tensile properties of electrospun nylon 6 single nanofibers. J. Polym. Sci. Part B Polym. Phys. 2010, 48, 1719-1731.

67. Zussman, E.; Burman, M.; Yarin, A.; Khalfin, R.; Cohen, Y. Tensile deformation of electrospun nylon-6, 6 nanofibers. J. Polym. Sci. Part B Polym. Phys. 2006, 44, 1482-1489.

68. Sanatgar, R.H.; Borhani, S.; Ravandi, S.A.H.; Gharehaghaji, A.A. The influence of solvent type and polymer concentration on the physical properties of solid state polymerized PA66 nanofiber yarn. J. Appl. Polym. Sci. 2012, 126, 1112-1120.

69. Hang, F.; Lu, D.; Bailey, R.J.; Jimenez-Palomar, I.; Stachewicz, U.; Cortes-Ballesteros, B.; Davies, M.; Zech, M.; Bödefeld, C.; Barber, A.H. In situ tensile testing of nanofibers by combining atomic force microscopy and scanning electron microscopy. Nanotechnology 2011, 22, 365708 .

70. Stachewicz, U.; Peker, I.; Tu, W.; Barber, A.H. Stress delocalization in crack tolerant electrospun nanofiber networks. ACS Appl. Mater. Interfaces 2011, 3, 1991-1996.

71. Veleirinho, B.; Rei, M.F.; Lopes-DA-Silva, J. Solvent and concentration effects on the properties of electrospun poly (ethylene terephthalate) nanofiber mats. J. Polym. Sci. Part B Polym. Phys. 2008, 46, 460-471.

72. Zong, X.; Ran, S.; Fang, D.; Hsiao, B.S.; Chu, B. Control of structure, morphology and property in electrospun poly (glycolide-co-lactide) non-woven membranes via post-draw treatments. Polymer 2003, 44, 4959-4967.

73. Wu, S.Z.; Yang, X.P.; Zhang, F.; Hou, X.X. Stretching-induced orientation for improving the mechanical properties of electrospun polyacrylonitrile nanofiber sheet. Adv. Mater. Res. 2008, 47, 1169-1172.

74. Kongkhlang, T.; Tashiro, K.; Kotaki, M.; Chirachanchai, S. Electrospinning as a new technique to control the crystal morphology and molecular orientation of polyoxymethylene nanofibers. J. Am. Chem. Soc. 2008, 130, 15460-15466.

75. Huang, C.; Chen, S.; Reneker, D.H.; Lai, C.; Hou, H. High-Strength Mats from Electrospun Poly ( $p$-Phenylene Biphenyltetracarboximide) Nanofibers. Adv. Mater. 2006, 18, 668-671.

76. Mohan, S.D.; Mitchell, G.R.; Davis, F.J. Chain extension in electrospun polystyrene fibres: A SANS study. Soft Matter 2011, 7, 4397-4404.

77. Tan, E.; Lim, C. Physical properties of a single polymeric nanofiber. Appl. Phys. Lett. 2004, 84, 1603-1605.

78. Lim, C.; Tan, E.; Ng, S. Effects of crystalline morphology on the tensile properties of electrospun polymer nanofibers. Appl. Phys. Lett. 2008, 92, 141908-141908-141903.

79. Bashur, C.A.; Dahlgren, L.A.; Goldstein, A.S. Effect of fiber diameter and orientation on fibroblast morphology and proliferation on electrospun poly (D,L-lactic-co-glycolic acid) meshes. Biomaterials 2006, 27, 5681-5688.

80. Pai, C.-L.; Boyce, M.C.; Rutledge, G.C. Mechanical properties of individual electrospun PA 6(3)T fibers and their variation with fiber diameter. Polymer 2011, 52, 2295-2301. 
81. Chew, S.Y.; Hufnagel, T.C.; Lim, C.T.; Leong, K.W. Mechanical properties of single electrospun drug-encapsulated nanofibres. Nanotechnology 2006, 17, 3880.

82. Shin, M.K.; Kim, S.I.; Kim, S.J.; Kim, S.-K.; Lee, H.; Spinks, G.M. Size-dependent elastic modulus of single electroactive polymer nanofibers. Appl. Phys. Lett. 2006, 89, 231923-231929.

83. Fennessey, S.F.; Farris, R.J. Fabrication of aligned and molecularly oriented electrospun polyacrylonitrile nanofibers and the mechanical behavior of their twisted yarns. Polymer 2004, 45, 4217-4225.

84. Kim, J.S.; Reneker, D.H. Polybenzimidazole nanofiber produced by electrospinning. Polym. Eng. Sci. 1999, 39, 849-854.

85. Stachewicz, U.; Bailey, R.J.; Wang, W.; Barber, A.H. Size dependent mechanical properties of electrospun polymer fibers from a composite structure. Polymer 2012, 53, 5132-5137.

86. Schellekens, R.; Bastiaansen, C. The drawing behavior of polyvinylalcohol fibers. J. Appl. Polym. Sci. 1991, 43, 2311-2315.

87. Arinstein, A.; Zussman, E. Electrospun polymer nanofibers: Mechanical and thermodynamic perspectives. J. Polym. Sci. Part B Polym. Phys. 2011, 49, 691-707.

88. Arinstein, A.; Burman, M.; Gendelman, O.; Zussman, E. Effect of supramolecular structure on polymer nanofibre elasticity. Nat. Nanotechnol. 2007, 2, 59-62.

89. Naraghi, M.; Arshad, S.; Chasiotis, I. Molecular orientation and mechanical property size effects in electrospun polyacrylonitrile nanofibers. Polymer 2011, 52, 1612-1618.

90. Papkov, D.; Zou, Y.; Andalib, M.N.; Goponenko, A.; Cheng, S.Z.; Dzenis, Y.A. Simultaneously Strong and Tough Ultrafine Continuous Nanofibers. ACS Nano 2013, 7, 3324-3331.

91. Allen, R.; Ward, I.; Bashir, Z. An investigation into the possibility of measuring an 'X-ray modulus' and new evidence for hexagonal packing in polyacrylonitrile. Polymer 1994, 35, 2063-2071.

92. Sawai, D.; Fujii, Y.; Kanamoto, T. Development of oriented morphology and tensile properties upon superdawing of solution-spun fibers of ultra-high molecular weight poly (acrylonitrile). Polymer 2006, 47, 4445-4453.

93. Brandrup, J.; Immergut, E.H.; Grulke, E.A.; Abe, A.; Bloch, D.R. Polymer Handbook, 4th ed.; John Wiley \& Sons: New York, NY, USA, 1999; Vol. 5, p. 61.

94. Lewin, M. Handbook of Fiber Chemistry, 3rd Ed. ed; Taylor \& Francis Group: Boca Raton, FL, USA, 2006; pp. 812-958.

95. Bisschops, J. Gelation of concentrated polyacrylonitrile solutions. II. J. Polym. Sci. 1955, 17, 89-98.

96. Cleland, R.L.; Stockmayer, W.H. An intrinsic viscosity-molecular weight relation for polyacrylonitrile. J. Polym. Sci. 1955, 17, 473-477.

97. Reneker, D.H.; Yarin, A.L.; Fong, H.; Koombhongse, S. Bending instability of electrically charged liquid jets of polymer solutions in electrospinning. J. Appl. Phys. 2000, 87, 4531.

98. Theron, S.; Zussman, E.; Yarin, A. Experimental investigation of the governing parameters in the electrospinning of polymer solutions. Polymer 2004, 45, 2017-2030. 
99. Richard-Lacroix, M.; Pellerin, C. Molecular Orientation in Electrospun Fibers: From Mats to Single Fibers. Macromolecules 2013, 46, 9473-9493.

100. Srinivasan, G.; Reneker, D.H. Structure and morphology of small diameter electrospun aramid fibers. Polym. Int. 1995, 36, 195-201.

101. Huang, C.; Wang, S.; Zhang, H.; Li, T.; Chen, S.; Lai, C.; Hou, H. High strength electrospun polymer nanofibers made from BPDA-PDA polyimide. Eur. Polym. J. 2006, 42, 1099-1104.

102. Chen, F.; Peng, X.; Li, T.; Chen, S.; Wu, X.-F.; Reneker, D.H.; Hou, H. Mechanical characterization of single high-strength electrospun polyimide nanofibres. J. Phys. D Appl. Phys. 2008, 41, 025308.

103. Kaneda, T.; Katsura, T.; Nakagawa, K.; Makino, H.; Horio, M. High-strengthhigh-modulus polyimide fibers I. One-step synthesis of spinnable polyimides. J. Appl. Polym. Sci. 1986, 32, 3133-3149.

104. Baughman, R.H.; Zakhidov, A.A.; de Heer, W.A. Carbon nanotubes-The route toward applications. Science 2002, 297, 787-792.

105. Thostenson, E.T.; Ren, Z.; Chou, T.-W. Advances in the science and technology of carbon nanotubes and their composites: a review. Compos. Sci. Technol. 2001, 61, 1899-1912.

106. Hussain, F.; Hojjati, M.; Okamoto, M.; Gorga, R.E. Review article: Polymer-matrix nanocomposites, processing, manufacturing, and application: An overview. J. Compos. Mater. 2006, 40, 1511-1575.

107. Yu, M.-F.; Files, B.S.; Arepalli, S.; Ruoff, R.S. Tensile loading of ropes of single wall carbon nanotubes and their mechanical properties. Phys. Rev. Lett. 2000, 84, 5552.

108. Shenderova, O.; Brenner, D.; Ruoff, R.S. Would diamond nanorods be stronger than fullerene nanotubes? Nano Lett. 2003, 3, 805-809.

109. Biercuk, M.; Llaguno, M.C.; Radosavljevic, M.; Hyun, J.; Johnson, A.T.; Fischer, J.E. Carbon nanotube composites for thermal management. Appl. Phys. Lett. 2002, 80, 2767-2769.

110. Xie, X.-L.; Mai, Y.-W.; Zhou, X.-P. Dispersion and alignment of carbon nanotubes in polymer matrix: a review. Mater. Sci. Eng. R Rep. 2005, 49, 89-112.

111. Grossiord, N.; Loos, J.; Regev, O.; Koning, C.E. Toolbox for dispersing carbon nanotubes into polymers to get conductive nanocomposites. Chem. Mater. 2006, 18, 1089-1099.

112. Salalha, W.; Dror, Y.; Khalfin, R.L.; Cohen, Y.; Yarin, A.L.; Zussman, E. Single-walled carbon nanotubes embedded in oriented polymeric nanofibers by electrospinning. Langmuir 2004, 20, 9852-9855.

113. Wan, Y.Q.; He, J.H.; Yu, J.Y. Carbon nanotube-reinforced polyacrylonitrile nanofibers by vibration-electrospinning. Polym. Int. 2007, 56, 1367-1370.

114. Kannan, P.; Eichhorn, S.J.; Young, R.J. Deformation of isolated single-wall carbon nanotubes in electrospun polymer nanofibres. Nanotechnology 2007, 18, 235707.

115. Sen, R.; Zhao, B.; Perea, D.; Itkis, M.E.; Hu, H.; Love, J.; Bekyarova, E.; Haddon, R.C. Preparation of single-walled carbon nanotube reinforced polystyrene and polyurethane nanofibers and membranes by electrospinning. Nano Lett. 2004, 4, 459-464. 
116. Baji, A.; Mai, Y.-W.; Wong, S.-C.; Abtahi, M.; Du, X. Mechanical behavior of self-assembled carbon nanotube reinforced nylon 6, 6 fibers. Compos. Sci. Technol. 2010, 70, 1401-1409.

117. Hou, H.; Ge, J.J.; Zeng, J.; Li, Q.; Reneker, D.H.; Greiner, A.; Cheng, S.Z. Electrospun polyacrylonitrile nanofibers containing a high concentration of well-aligned multiwall carbon nanotubes. Chem. Mater. 2005, 17, 967-973.

118. Jose, M.V.; Steinert, B.W.; Thomas, V.; Dean, D.R.; Abdalla, M.A.; Price, G.; Janowski, G.M. Morphology and mechanical properties of Nylon 6/MWNT nanofibers. Polymer 2007, 48, 1096-1104.

119. Lu, P.; Hsieh, Y.-L. Multiwalled carbon nanotube (MWCNT) reinforced cellulose fibers by electrospinning. ACS Appl. Mater. Interfaces 2010, 2, 2413-2420.

120. Wang, W.; Ciselli, P.; Kuznetsov, E.; Peijs, T.; Barber, A. Effective reinforcement in carbon nanotube-polymer composites. Philos. Trans. R. Soc. A Math. Phys. Eng. Sci. 2008, 366, 1613-1626.

121. Wang, Z.; Ciselli, P.; Peijs, T. The extraordinary reinforcing efficiency of single-walled carbon nanotubes in oriented poly (vinyl alcohol) tapes. Nanotechnology 2007, 18, 455709.

122. Inagaki, M.; Yang, Y.; Kang, F. Carbon nanofibers prepared via electrospinning. Adv. Mater. 2012, 24, 2547-2566.

123. Kim, C.; Yang, K.S.; Kojima, M.; Yoshida, K.; Kim, Y.J.; Kim, Y.A.; Endo, M. Fabrication of Electrospinning-Derived Carbon Nanofiber Webs for the Anode Material of Lithium-Ion Secondary Batteries. Adv. Funct. Mater. 2006, 16, 2393-2397.

124. Zussman, E.; Chen, X.; Ding, W.; Calabri, L.; Dikin, D.; Quintana, J.; Ruoff, R. Mechanical and structural characterization of electrospun PAN-derived carbon nanofibers. Carbon 2005, 43, 2175-2185.

125. Zussman, E.; Yarin, A.L.; Bazilevsky, A.V.; Avrahami, R.; Feldman, M. Electrospun Polyaniline/Poly (methyl methacrylate)-Derived Turbostratic Carbon Micro-/Nanotubes. Adv. Mater. 2006, 18, 348-353.

126. Kim, C.; Jeong, Y.I.; Ngoc, B.T.N.; Yang, K.S.; Kojima, M.; Kim, Y.A.; Endo, M.; Lee, J.W. Synthesis and characterization of porous carbon nanofibers with hollow cores through the thermal treatment of electrospun copolymeric nanofiber webs. Small 2007, 3, 91-95.

127. Zhou, Z.; Lai, C.; Zhang, L.; Qian, Y.; Hou, H.; Reneker, D.H.; Fong, H. Development of carbon nanofibers from aligned electrospun polyacrylonitrile nanofiber bundles and characterization of their microstructural, electrical, and mechanical properties. Polymer 2009, 50, 2999-3006.

128. Park, S.H.; Kim, C.; Choi, Y.O.; Yang, K.S. Preparations of pitch-based CF/ACF webs by electrospinning. Carbon 2003, 41, 2655-2657.

129. Park, S.H.; Kim, C.; Yang, K.S. Preparation of carbonized fiber web from electrospinning of isotropic pitch. Synth. Met. 2004, 143, 175-179.

130. Zou, L.; Gan, L.; Lv, R.; Wang, M.; Huang, Z.-h.; Kang, F.; Shen, W. A film of porous carbon nanofibers that contain $\mathrm{Sn} / \mathrm{SnO}_{X}$ nanoparticles in the pores and its electrochemical performance as an anode material for lithium ion batteries. Carbon 2011, 49, 89-95. 
131. Xuyen, N.T.; Ra, E.J.; Geng, H.-Z.; Kim, K.K.; An, K.H.; Lee, Y.H. Enhancement of conductivity by diameter control of polyimide-based electrospun carbon nanofibers. J. Phys. Chem. B 2007, 111, 11350-11353.

132. Merino, C.; Soto, P.; Vilaplana-Ortego, E.; Gomez de Salazar, J.M.; Pico, F.; Rojo, J.M. Carbon nanofibres and activated carbon nanofibres as electrodes in supercapacitors. Carbon 2005, 43, 551-557.

133. Kim, C.; Kim, J.-S.; Kim, S.-J.; Lee, W.-J.; Yang, K.-S. Supercapacitors prepared from carbon nanofibers electrospun from polybenzimidazol. J. Electrochem. Soc. 2004, 151, A769-A773.

134. Arshad, S.N.; Naraghi, M.; Chasiotis, I. Strong carbon nanofibers from electrospun polyacrylonitrile. Carbon 2011, 49, 1710-1719. 


\title{
The Role of the Protein Corona in Fiber Structure-Activity Relationships
}

\author{
Melanie Kucki, Jean-Pierre Kaiser, Martin J. D. Clift, \\ Barbara Rothen-Rutishauser, Alke Petri-Fink and Peter Wick
}

\begin{abstract}
When nanomaterials enter biological fluids, they are immediately covered by biomolecules, particularly proteins, forming the so-called protein corona. The dynamic nature and complexity of the protein corona can impact upon the biological effects and distribution of nanomaterials with an organism. Therefore, the protein corona is an important factor in determining the biological impact of any nanomaterials. The protein adsorption pattern is determined by various factors, including the bio-fluids' protein composition, the nanomaterials' physicochemical properties, as well as the time and type of exposure. Predominantly, research has focused upon spherical nano-objects, however, due to their ever-increasing potential use within human based applications, and, therefore, heightening and inevitable exposure to the human body, little is known regarding how proteins interact with nanofibers. Therefore, the present review focuses on the current knowledge as to how the geometry of man-made (nano)fibers, carbon nanotubes (in comparison with asbestos fibers), affects their interaction with proteins within biological fluids. Summarizing state-of the art methodologies applied to dissect protein-binding signatures, it is further discussed whether the protein corona composition of fibrous and non-fibrous materials differ, as well as what impact the protein corona has on (nano)fiber uptake, intracellular distribution and their subsequent toxicity.
\end{abstract}

Reprinted from Fibers. Cite as: Kucki, M.; Kaiser, J.-P.; Clift, M.J.D.; Rothen-Rutishauser, B.; Petri-Fink, A.; Wick, P. The Role of the Protein Corona in Fiber Structure-Activity Relationships. Fibers 2014, 2, 187-210.

\section{Introduction}

As soon as nanomaterials come into contact with biological fluids they are immediately covered by a large range of different biomolecules, such as proteins, peptides, and lipids. As this bio-corona is composed of proteins and peptides to a high extent, it is often referred as the protein-corona. The composition of the protein corona underlies certain dynamic fluctuations. Most importantly, it is widely accepted that the composition of the protein corona determines the biological identity of any (nano)material, and the resultant biological activity and distribution [1]. Various studies have been performed to identify the composition of the protein corona of a range of nanomaterials and to correlate the obtained protein adsorption pattern to the physicochemical properties of the materials, as reviewed by $[2,3]$ and others. 
The aim of the investigation into the protein corona is to enable the correlation of the physicochemical parameters of a (nano)material type to the composition (quantitatively and qualitatively) of the protein corona, and to the resulting shortand long-term biological response. The goal is to obtain a biological fingerprint, or barcode of the individual (nano)materials. This, in turn, would enable to estimate or even predict the biological effects of the material, ranging from the level of toxicity to the efficiency as diagnostic tools or drug carriers. This is of importance for (nano)safety issues, as well as for pharmaceutical engineering.

Most studies on the composition of the protein corona have been performed on spherical (nano)particles. However, there are also several studies available that focused on the protein adsorption to fibrous materials. Due to their toxicological relevance toward humans, especially asbestos and carbon nanotubes (CNTs) have come under intense investigation.

Asbestos can be classified as a group of six silicate minerals: amosite, chrysotile, crocidolite, as well as actinolite, anthophyllite, and tremolite. Due to its high temperature and chemical resistance, in combination with its tensile strength, asbestos has been widely used within a large number of applications, e.g., as insulating material in building construction. Although, due to the severe health effects that have been shown to be caused by asbestos-related exposures (i.e., asbestosis, mesothelioma; it is defined as a human 1A carcinogen by the International Agency for Research on Cancer (IARC)/World Health Organization (WHO), the use of asbestos has undergone heightened regulations for its use, and furthermore banned from being used within numerous countries throughout the world.

Carbon nanotubes (CNTs), fibrous carbon allotropes, have been under intense investigation since their detailed description in 1991 [4]. Their diameter ranges from a few to several nanometers, to a length of up to several millimeters. The CNTs possess unique properties, such as tensile strength, high stiffness, temperature resistance, chemical resistance, and water resistance, amongst others. Due to these advantages these substances found various applications as reinforcement in polymers, in technical textiles, as sound absorbers, in cold-, heat- and fire protectors, and in medical applications.

\section{Human Exposure to (Nano)fibers}

Due to their physical properties some fibrous materials, such as asbestos fibers, have shown a severe impact towards human health, which also has been described in the literature as contributing to the fiber paradigm [5]. According to this, fibers that are thin enough or have an aerodynamic diameter small enough to enter the lung and which are too rigid and long to be taken up by phagocytes, have a high potential to damage the lung tissue, which can lead up to severe health effects based on persistence of the material within the lung. 
For example, inhalation of asbestos fibers for prolonged periods has been clearly shown to be the specific cause of serious health effects, such as asbestosis and the development of malignant mesothelioma. Furthermore, erionite, a natural zeolite fiber, has been classified as a human carcinogen and is also reported to cause mesothelioma following long-term exposure [6,7].

In general, there are three main routes for fibers to enter the human body: by inhalation, by ingestion, or via penetration of the skin. The relevance of these portals of entry is dependent upon the kind of exposure scenario. Nonetheless, the human lung remains to be considered the primary portal of entry for any fibrous material. Entering the upper airways, fibers can be enveloped by mucus and cleared by mucociliar transportation, being coughed out or, alternatively they can be swallowed down into the stomach. It is assumed that most fibers with an aerodynamic diameter of a few micrometers are also able to reach the lower, non-ciliated airways (i.e., the alveolar region; responsible for gas exchange in the alveoli; Figure 1) The human lung consists of around 300 million alveoli, each with a diameter in the range of $200 \mu \mathrm{m}$. The air in the lumen of the alveoli has a close proximity (i.e., 100 nm) from the bloodstream. The highly available surface for gaseous exchange at this air-blood tissue barrier is around $140 \mathrm{~m}^{2}$ [8-10]. This surface is covered by the lung lining fluid or so-called surfactant, which is secreted by the lung epithelial cells. The lung lining fluid contributes to host defense, as well as regulates the surface tension at the air-blood barrier. Entering the alveoli, fibers and other foreign materials are coated with proteins (e.g., surfactant proteins), peptides, and lipids of the lung lining fluid. Although, the presence of over 40 different cell types, including important barrier cells types (epithelial and endothelial cells) [11], contribute to the normal homeostasis of this region following deposition of foreign materials, it is the interaction between important immune cells, specifically macrophages [12], and dendritic cells [13] that allows for the clearance of such materials. This clearance is, however, much slower (days to weeks) in comparison to the fast mucociliar clearance in the airways (minutes to hours). It is important to note, that although these lung defense mechanisms are highly active and efficient in removing foreign deposited materials, fibrous materials, due to their high aspect ratio, can have a different mode of action compared to non-fibrous particles.

Long, thin, and non-flexible (i.e., stiff) fibers are unable to be completely engulfed by lung (i.e., alveolar) macrophages and can incite the phenomenon known as "frustrated phagocytosis" [14,15]. If these materials are non-degradable, they will persist in the lungs (i.e., become bio-persistent) and can elicit a persistent inflammatory response. Prolonged exposure can therefore lead to an accumulation of these materials in the lung, which in turn can lead to severe health effects as the development of fibrosis and cancer [16]. For a complete review regarding the biological impact of fibers and nanofibers, please refer to [5,17]. 


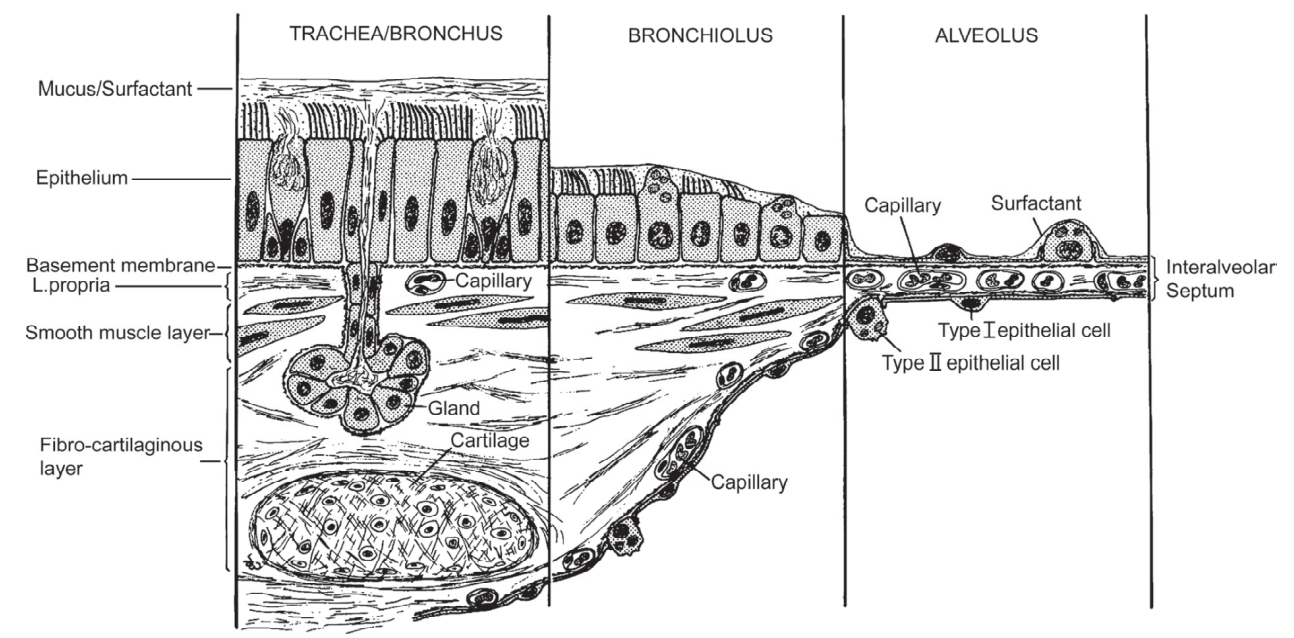

Figure 1. Drawing of the airway wall structure at the three principal levels from [11].

As mentioned above, most forms of asbestos and erionite fibers are examples of fibrous materials that are known to cause the development of mesothelioma following prolonged exposure periods. Such effects are only known nowadays due to years of intense scientific research following unfortunate reports of worker ill-health in the asbestos industry since the 1950s. Therefore, due to the fibrous morphology of CNTs and their widespread use, safety concerns have been raised, leading to the discussion on the potential biological impact deriving from CNT human exposure. Over the past two decades, increased research has been performed on this issue [18]. Briefly, it has been reported that short, flexible and entangled CNT can be internalized by macrophages and cleared from the lungs. In contrast, stiff and straight CNTs might cause asbestos-like pathogenic effects [19]. Frustrated phagocytosis as well as simultaneous phagocytosis of single fibers by several macrophages could be observed [20]. By logistic regression models it could be demonstrated that differences in carcinogenicity of a fiber is a function of fiber characteristics, dimensions (thickness, length) and persistence in the lungs [21]. Fiber dimensions (thinner than $1 \mu \mathrm{m}$ and longer than $20 \mu \mathrm{m}$ ) and the dissolution rate were the most important parameters in initiating malignant lung tumors and mesothelioma [22]. However, long, bio-soluble fibers such as rock-wool exhibit low pathogenic potential [23]. As there is no threshold for cancerous substances, there is no specific "sub-critical" dose. The risk therefore, to develop cancer can be estimated by the exposure duration and the amount (number) of inhalable fibers. Therefore, in theory, even low amounts and short exposure times can be sufficient enough to trigger cancer development $[24,25]$. 
Despite the increased research into the biological impact of fibers and nanofibers, the driven cellular interaction of these fibers is not solely related to their physical characteristics, but a combination with their interaction with the biological environment, i.e., their presence within biological fluid (e.g., biomolecules (proteins, enzymes, lipids) prior to any cellular entity. Thus, when considering the inhalation of any fibrous material, assuming that it deposits within the alveolar region of the human lung, this deposited fraction will primarily interact with the pulmonary surfactant layer, a mixture of surfactant proteins and lipids, and the underlying aqueous phase, prior to any subsequent interaction with the cells of the epithelial airway barrier. At this point, complex competitive ad- and de-sorption processes will lead to the formation of certain protein adsorption patterns, which have been shown to be dependent on the physicochemical properties of the (nano)materials, as well as the structure and affinity of the proteins [26].

The aim of the present review is therefore, to highlight the role of the protein corona in the fiber structure-activity relationship. Especially both asbestos fibers and carbon nanotubes, due to their toxicological relevance towards humans, will be focused upon. This review will critically analyze the existing literature describing the interaction between fibers and proteins and their influence on cellular uptake, biological response (including adverse effects), as well as bioavailability. A short overview on the methods applied to investigate protein profiles is given within the next section to show how these profiles are obtained and where further progress might be needed to improve our understanding of the protein corona of different nanomaterials. This is a general section valid for the investigation of protein coronas of different nanomaterials, not only fiber materials.

\section{Methods to Determine the Protein Coating of (Nano)materials}

To gain insight into the mechanisms of protein binding at the solid-liquid interface, different approaches have been developed. In principle, they can be divided into single-protein studies, studies performed in complex medium, as well as mathematical modeling. To investigate the protein corona of a (nano)material in a complex medium, the main challenges of these analyses are the high complexity of biomolecules, as well as the highly dynamic nature of proteinnanomaterial interactions.

To investigate the protein-(nano)material interaction in complex medium, nanomaterials are incubated with biological fluids at protein concentrations that, in an ideal case, resemble the protein concentrations within the biological environment. After incubation, most approaches performed so far include a separation of excess non-bound proteins. For the separation of the protein-nanomaterial complexes from excess proteins, several techniques can be applied, including microfiltration, dialysis, magnetic separation, and centrifugation. With each method, after protein separation 
and several washing steps, the bound proteins are released from the nanomaterial surface by adding a denaturing buffer, separated from each other and identified by liquid chromatography coupled mass spectroscopy (LC-MS) after trypsin treatment. This procedure does not take into account the dynamics of these protein fiber interactions and mostly represent a situation which is thermodynamically equilibrated and not representative of realistic physiological conditions.

The most commonly used method to separate the different proteins is one- or two-dimensional polyacrylamide gel electrophoresis (1D/2D-PAGE). In this method, proteins are identified according to their molecular weight or isoelectric point. To identify the proteins, the protein pattern on the protein gels can be compared to so-called master maps obtained from human plasma proteins or analyzed by MS [27]. Proteins are also separated by LC, size exclusion chromatography (SEC) or affinity chromatography. This can be performed on individual excised protein bands obtained by 1D/2D-PAGE, or directly coupled to liquid chromatography as so-called LC-MS or by Matrix-assisted laser desorption/ionization (MALDI) analysis combined with mass spectrometry with time of flight (TOF) analysis. The experimental mass spectra are analyzed and compared against data from protein sequence databases (e.g., UniProtKB/Swiss Prot, web.expasy.org). Additionally, these protein sequences can be identified by $\mathrm{N}$-terminal sequencing [28]. LC-MS is accepted as the most developed and commonly used method, therefore, the most relevant, although MALDI-TOF can be considered as the most sensitive method.

In general, the number of proteins reported to be associated with nanomaterials varies in the order of one magnitude. This variation is primarily based on the methodological approach of the analysis and therefore the relevant detection limits of the procedure and the equipment used $[29,30]$. Several studies have shown that protein binding to material surfaces is highly dependent on the ionic strength of the surrounding medium. Therefore, the type of buffers applied in experimental protocols can have a significant effect on the end result. It has been shown that the duration, volume and number of washing steps can affect the resulting protein profile, especially the reported values of albumin. Sempf et al. [27,29] suggested that high abundance proteins like albumin can occur as contaminations, but are not associated with the protein corona. In addition, Sempf and colleagues compared the available data on the protein adsorption patterns of a diversity of polymeric particles obtained by 2D-PAGE. Despite differences in body distribution based on material surface properties, similar proteins were reported to be in the protein corona. The authors therefore concluded that this is caused by a lack of sensitivity of the analytical method applied [27].

In summary, several methods are available to investigate the composition of the protein corona, each with its limitations. Further development of the analytic 
methods is necessary, especially those enabling time-dependent resolution, in order to gain a full understanding of the highly complex protein-(nano)material interactions.

\section{Interactions of Proteins and Fibers}

\subsection{Asbestos-Protein Interactions}

Most of the studies on the protein-material interactions of asbestos fibers available are single-protein studies elaborating the interactions of different proteins and asbestos fiber materials (Table 1). Only a few studies are available investigating protein adsorption onto asbestos fibers in complex protein mixtures. Even if single protein studies cannot give information about the adsorption behavior of a protein in a complex mixture, these studies are necessary to elaborate the general mechanisms of protein adsorption to fibers. Morgan et al. [31] investigated in the adsorption of human serum albumin on different types of asbestos fiber types (chrysotile, crocidolite, amosite, anthophyllite). It was reported that the difference in protein adsorption was due mainly to charge differences of the fibers' surface. However, notable studies a few years later showed that hydrophobic interactions, nature and density of functional groups, as well as the absence or presence of ionic species significantly contributed to the protein adsorption upon asbestos fibers. In 1977, Light et al. [32] showed that by leaching of magnesium from the fibers, the surface charge of chrysotile and crocidolite fibers can be altered. Light and colleagues observed a significant correlation between the change in surface charge and the hemolytic activity of the fibers. These results were confirmed by Morgan et al. [33] who further demonstrated that $\mathrm{Mg}^{2+}$-depletion of chrysotile fibers influences the albumin binding capacity of the fiber material, as well as the selective release of acid hydrolases from mouse peritoneal macrophages and the incidence of mesothelial tumors in rats. These findings show that the surface characteristics of a fiber material can lead to a change in protein adsorption, which in turn can influence the biological effects. 
Table 1. Key citations published in the last 40 years regarding the asbestos-protein interaction. Please note that this table is not a complete list, however highlights the important results concerning asbestos-biomolecule related research.

\begin{tabular}{|c|c|c|c|c|}
\hline $\begin{array}{l}\text { Year of } \\
\text { Publication }\end{array}$ & Type of Study & $\begin{array}{l}\text { Type of } \\
\text { Adsorbed } \\
\text { Protein(s) }\end{array}$ & Major Outcome & Reference \\
\hline 1974 & $\begin{array}{l}\text { Protein } \\
\text { adsorption }\end{array}$ & $\begin{array}{l}\text { Human serum } \\
\text { albumin }\end{array}$ & $\begin{array}{l}\text { The surface charge of the asbestos } \\
\text { fibers had a strong influence on } \\
\text { the adsorption of proteins. }\end{array}$ & [31] \\
\hline 1977 & In vitro, in vivo & $\begin{array}{l}\text { Human serum } \\
\text { albumin }\end{array}$ & $\begin{array}{l}\text { The capacity of asbestos fibers to } \\
\text { adsorb proteins is dependent } \\
\text { from the magnesium content in } \\
\text { the fibers. }\end{array}$ & [33] \\
\hline 1980 & $\begin{array}{l}\text { Protein } \\
\text { adsorption }\end{array}$ & $\begin{array}{l}\text { Bovine serum } \\
\text { albumin, Ferritin }\end{array}$ & $\begin{array}{l}\text { Magnesium depletion of the } \\
\text { asbestos fibers leads to a decrease } \\
\text { of albumin adsorption, while the } \\
\text { specific adsorption offerritin } \\
\text { increased. }\end{array}$ & [34] \\
\hline 1986 & $\begin{array}{l}\text { Protein } \\
\text { adsorption }\end{array}$ & $\begin{array}{l}\text { Fetal serum } \\
\text { proteins }\end{array}$ & $\begin{array}{l}\text { Strong electrostatic interactions } \\
\text { between the charges of the fibers } \\
\text { and the proteins were responsible } \\
\text { for the protein-fiber adsorption. }\end{array}$ & [35] \\
\hline 1987 & $\begin{array}{l}\text { Protein } \\
\text { adsorption }\end{array}$ & $\begin{array}{l}\text { Different types of } \\
\text { proteins }\end{array}$ & $\begin{array}{l}\text { The protein-fiber affinity was } \\
\text { correlated with the specific area of } \\
\text { the fiber and the protein charge } \\
\text { density. }\end{array}$ & [36] \\
\hline \multirow[t]{2}{*}{1990} & In vitro & Serum proteins & $\begin{array}{l}\text { The cytotoxic effects of asbestos } \\
\text { fibers was serum-dose dependent. }\end{array}$ & [37] \\
\hline & & $\begin{array}{l}\text { Immunoglobulin } \\
\text { G, Bovine serum } \\
\text { albumin, } \\
\text { Cytochrome c }\end{array}$ & $\begin{array}{l}\text { Certain proteins were selectively } \\
\text { adsorbed onto the asbestos fibers. }\end{array}$ & [38] \\
\hline 1995 & In vitro & $\begin{array}{l}\text { Vitronectin, } \\
\text { Fibronectin }\end{array}$ & $\begin{array}{l}\text { Vitronectin specifically enhanced } \\
\text { the internalization of asbestos } \\
\text { fibers via } \alpha \mathrm{v} \beta 5 \text { integrin receptors. }\end{array}$ & [39] \\
\hline \multirow[t]{2}{*}{2000} & In vitro & Vitronectin & $\begin{array}{l}\text { The adsorption of vitronectin } \\
\text { onto the asbestos fibers increased } \\
\text { the fiber uptake and the cytotoxic } \\
\text { effects of asbestos. }\end{array}$ & [40] \\
\hline & & & $\begin{array}{l}\text { Vitronectin adsorption to } \\
\text { chrysotile asbestos fibers } \\
\text { increased fiber phagocytosis and } \\
\text { toxicity for mesothelial cells. }\end{array}$ & [41] \\
\hline
\end{tabular}

To determine whether there is a preferential adsorption of certain proteins in a complex mixture (such as blood or serum containing cell culture medium), protein adsorption on asbestos fibers in fetal calf serum was investigated by Valerio 
and co-workers. Compared to their abundance in serum, an enrichment of certain proteins on the fibers was found. Based on their results on different asbestos fiber types, Valerio et al. [35] concluded that the fiber dimensions is an important factor regarding the onset of cancer, but also, concomitantly, the specific protein adsorption characteristics to a fibrous material is a contributing factor to this biological effect. Boylan et al. [39] subsequently showed that vitronectin, a $75 \mathrm{kDa}$ glycoprotein abundant in serum, can be easily adsorbed to crocidolite fibers. It was further noted that by pre-coating crocidolite fibers with vitronectin it significantly increases the internalization of these asbestos fibers by rabbit pleural mesothelial cells. Pre-coating of crocidolite with serum, which naturally contains vitronectin, had similar effects, whereas vitronectin-depleted serum did not lead to enhanced fiber internalization. Similar effects were also observed by Boylan and colleagues with vitronectin-coated chrysotile fibers underlying the importance of this finding. Despite the role of proteins in determining their internalization, or not, it was still noted that the associated hazard with asbestos fibers remained, with intracellular oxidation, DNA strand breaks, cell-cycle arrest, and apoptosis being observed under these conditions $[40,41]$. In summary, these results demonstrate that the presence of proteins on the surface of asbestos fibers can significantly contribute to the subsequent biological effect measured.

\subsection{CNT-Protein Interactions}

\subsubsection{Mechanism of Interaction}

The size, chemical, and surface properties of CNTs are completely different compared to all forms of asbestos fibers. CNTs consist ideally of pure carbon, hexagonally arranged and formed as a tube with one or more layers [18]. The manner in which proteins interact with these nanofibers is complex and specific to their surface features. Table 2 gives an overview on the key literature on CNT-protein interaction.

It was shown that the dispersibility of CNT in aqueous environment can be improved either by covalent functionalization, but also by non-covalent binding of proteins and surfactants to the CNT surface. In contrast to covalent functionalization, non-covalent binding of proteins retains valuable technological properties of the CNTs (optical, electronic and mechanical). Therefore, CNT-protein interactions were intensively studied in the past by intentional attachment of several proteins, peptides, or other biomolecules to the CNT surface.

It has previously been demonstrated that certain serum proteins, (e.g., albumin, fibrinogen and apolipoproteins) show a higher affinity to the hydrophobic CNT surface than the rest of serum proteins [42-45]. It was proposed that serum proteins bind to CNTs by non-covalent $\pi-\pi$ stacking hydrophobic interactions. Specifically, the 
interaction of aromatic residues of the proteins (e.g., phenylalanine, tryptophan, and tyrosine) with the hydrophobic surface of the CNTs was found to be important for the selective binding of proteins to CNTs [44,46-48]. Amphiphilic, $\alpha$-helical peptides adsorb with their hydrophobic regions of the helix onto the aromatic surface of the CNTs and the more polar residues are located against the aqueous environment. The non-covalent binding of the hydrophobic amino acids to single walled carbon nanotubes (SWCNT) initiated a de-bundling and an increased dispersion of the tubes in water [49-52]. Just recently, Sacchetti et al. [53] showed that the amount of adsorbed proteins is correlated with the total number of hydrophobic, aromatic protein residues. Proteins that possess hydrophobic binding sites were found to attach to the slightly curved hydrophobic $\pi$-electron-rich graphitic surface of the CNTs [54]. Thus, the fact that hydrophobic regions of proteins adsorb readily to CNTs had been used to coat and solubilize CNTs [55-57]. Another group of scientists could demonstrate that the adhesion force between proteins and CNTs was a function of the $\mathrm{pH}$. At low $\mathrm{pH}$ the protonated amine groups $\left(-\mathrm{NH}_{3}{ }^{+}\right)$of polylysine adsorbed strongly to the deprotonated carboxyl groups $\left(-\mathrm{COO}-+\mathrm{H}^{+}\right)$ of the oxidized (carboxylated) CNTs [58]. Salvador-Morales et al. [59] observed that binding of surfactant proteins to double walled carbon nanotubes (DWCNTs) was calcium-dependent. Control experiments in the absence of calcium ions showed no significant binding. Furthermore, comparison of different batches of pristine DWCNT showed that binding of surfactant proteins to DWCNT in the presence of calcium was linked to the presence of surface functional groups as carboxyl groups. Therefore, binding might be mediated by calcium-bridging.

Witus et al. [60] synthesized special peptides that bind non-covalently with disulphide bonds to functionalized SWCNTs in order to make them water soluble without altering their electronic structure. It is reported that proteins with a high content of basic residues, such as histones or lysozyme were suitable for the dispersion of CNTs. By this method, it was observed that the primary, secondary and tertiary protein structures play an essential role in forming a de-bundled CNT solution [61]. Proteins can bind with various amino acids to CNTs. By that, it is possible that the binding sequence is folding into a structure matching the geometry of the CNT to form a stable complex $[18,62,63]$. Conformational changes due to partial unfolding can lead to a reduction or complete blocking of the enzymatic activity [47]. There are several examples published reporting that enzymes showed a reduced activity or even a complete inhibition of the enzymatic activity after CNT-binding. Here some examples: Carboxyl-functionalized CNTs interacted with ribonuclease $\mathrm{A}$ and caused a reduction of the activity by a change of the protein conformation. The activity decreased further, when the enzyme adsorbed to larger CNTs [64]. Zhang et al. [65] also reported that different types of functionalized multi-walled CNTs (MWCNTs) were able to bind to, or near to the catalytic site of the 
digestive enzyme $\alpha$-chymotrypsin and inhibited its proteolytic activity completely. In a similar study it could be shown that the loss of activity was a function of the change in secondary structure upon adsorption of the proteins onto the surface of the SWCNTs $[66,67]$. On the other hand there are also studies that report that different metalloproteins immobilized on carboxylated SWCNTs did not show a detectable retention of their activity [68].

In summary, the adsorbed proteins onto the CNTs are affecting the CNT properties, as well as their behavior towards biological systems. However, in contrast to the asbestos-protein interaction, and despite an ever increasing understanding of the CNT-protein interaction, it is not currently possible to correlate how the proteins attached to the CNT surface effect the subsequent biological response observed. In order to achieve this, the biological effects of the protein corona on CNTs have to be investigated on a case-by-case level. It is hypothesized however, that the effects are dependent upon the type of CNT (size, diameter, curvature, functionalization, etc.), from the type of adsorbed proteins (isoelectric point, aromatic amino acids, hydrophobic binding sites, primary-, secondary-, and tertiary structure) and from the environment ( $\mathrm{pH}$, presence of other nanoparticles, etc.). Although further research must be performed to confirm this and provide definitive understanding to fully comprehend the nanosafety profile of CNTs.

\subsubsection{Influence of Solvents, Surfactants, Surface-Functionalization, and Pre-Coating on CNT-Protein Interactions}

It is well established that pre-coating of nanomaterials with certain molecules can influence the further protein binding pattern, as well as nanomaterial uptake and distribution. This has also been demonstrated for CNTs [69]. In a study, where double-walled CNTs came in contact with natural human lung surfactant, it was shown that surfactant protein A (SPA) and the SPD selectively bound onto the surface of the CNTs [59]. Surfactant proteins contribute towards the immune defense system at the epithelial airway barrier and further enhance the phagocytosis of antigens by (alveolar) macrophages. Further research that pre-coated bundled MWCNTs with porcine pulmonary surfactant $\left(\operatorname{Curosurf}^{\circledR}\right)$ found that this pro-protein corona affected the subsequent adsorption pattern of blood plasma proteins, as well as the cellular uptake by macrophages. It is important to note that whilst this protein coating had no effect on the cytotoxicity of the MWCNTs, it did mediate both a pro-inflammatory and oxidative stress effect in vitro. [70,71]. Further to this, studies by Holt et al. [72], investigated the internalization of bovine serum albumin (BSA) coated SWCNT. It was observed that cellular uptake of SWCNTs was proportional to the mass of SWCNT-BSA per cell. It could further be demonstrated that SWCNTs coated with BSA were internalized by different cell types, such as human mesenchymal stem cells or HeLa cells [73]. 
The protein-binding characteristics of nanomaterials can be significantly changed by surface functionalization or the addition of surface coatings [74]. To stabilize aqueous SWCNT dispersions, solvents or surfactant molecules are often applied [50]. Surfactant molecules consist of a hydrophilic head and a hydrophobic tail [75]. The adhesion of surfactants to the CNT is in principle similar to the adhesion of amphiphilic proteins as described above. Surfactant molecules adsorb with their hydrophobic tails onto the surfaces of the CNTs with the polar heads located against the aqueous environment. Dutta et al. [43] showed that pre-coating of SWCNT with the non-ionic amphiphilic copolymer surfactant Pluronic F127 reduced albumin adsorption.

For SWCNT that had been modified with polyethylene glycol (PEG), the pattern of adsorbed proteins was affected in dependence of the conformation of the PEG $[53,76]$. The natural protein binding affinity to SWCNTs is reduced or even eliminated by covalent functionalization of the CNTs with PEG moieties [77], which in turn changes the cellular uptake [42]. In another study, it has been demonstrated that pre-coating of SWCNT's with Triton X-100 prior to functionalization with PEG leads to a complete and uniform PEG-coating of the SWCNT sidewalls [78]. Practically no streptavidin or other proteins were able to adsorb onto SWCNT that had been treated with Triton X-100, as well as PEG. The importance here is if solvents or surfactants were used as a dispersion agent, these interactions ionic or non-ionic, rapidly or slowly exchanged by other compounds, such as proteins, has to be clarified in detailed in order to avoid misleading conclusions.

\subsubsection{Alternative Theory to CNT Protein Interactions}

In 2013, Cai et al. [79] released a study that was questioning the published theories concerning protein adsorption on CNTs surfaces. This was based on the fact that the authors did not observe a preference of hydrophobic protein moieties to bind onto MWCNTs. The hydrophobic aromatic amino acids (phenylalanine, tryptophan, and tyrosine) did not show higher affinity to CNTs than other amino acids. Interestingly another group made similar observations. Shannahan et al. [80] investigated the protein corona after incubating carboxylated CNTs (SWCNTs and MWCNTs), as well as pristine CNTs into fetal bovine serum. The quantities of adsorbed proteins on the carboxylated CNTs compared to the quantities of adsorbed proteins to pristine CNTs were similar. This indicates that hydrophobic interactions and $\pi-\pi$-stacking between the aromatic moieties of proteins and the CNTs did not play an important role in the CNT-protein interactions. In addition it could be shown that the carboxylated CNTs were able to bind a number of unique proteins such as HSp60 or Hsp70 which did not bind to the unmodified pristine CNTs. This implies that hydrogen bonding and electrostatic interactions as well as specific covalent bonding were involved. Further it had been proposed by Cai and co-workers [79] 
that the adsorbed protein binding patterns corresponded closely to the CNT-surface properties. MWCNTs with diameters of $20 \mathrm{~nm}$ to $40 \mathrm{~nm}$ or above were able to bind a significant amount of proteins. On the other hand, MWCNTs with diameters less than $10 \mathrm{~nm}$ and SWCNTs with diameters less than $2 \mathrm{~nm}$ showed no significant protein binding. Thus, the protein-CNT interaction depends on the size of the CNTs and on the three-dimensional arrangement of carbon atoms in the CNTs, and not on the chemical properties of carbon itself. Beside the diameter of the CNTs, a suitable surface curvature of the CNTs is required for a stronger protein binding [81]. Smoother curvature can induce larger protein conformational changes, while the protein adapts to the unfamiliar surface curvature. Peptides then re-orientate their structures to optimize their interactions with the SWCNTs through their aromatic residues [81].

From the available literature (Table 2) it can be summarized that protein-nanofiber interactions are highly dependent on various factors, which are (1) the inherent properties of the nanofiber (e.g., size, shape, curvature, diameter, surface-chemistry, zeta potential, density of functional surface groups, material composition, presence of impurities, surface functionalization/coating); (2) the properties of the proteins (e.g., size, isoelectric potential, primary, secondary and tertiary structure); (3) experimental/environmental conditions (e.g., type of dispersion medium, $\mathrm{pH}$, presence and absence of ionic species); and last, but not least, the presence and amount of other proteins or amino acids that compete for available surface for binding on the nanomaterial. Therefore, one binding mechanism can be dominant for a certain nanomaterial type, while another binding mechanism may become more relevant with changes in the materials' properties, proteins present (i.e., the biological environment). It is also important to note that the protein binding is a dynamic process and proteins can be exchanged constantly.

In summary, the reasons why the theories concerning the underlying mechanisms of protein-CNT interactions are quite diverse might be:

- Electrochemical and chemical nature of the CNT and proteins are essential for strong CNT-protein interaction

- Protein-CNT binding is based on non-covalent $\pi-\pi$ stacking hydrophobic interactions

- $\quad$ The diameter, size and surface curvature of the CNT is essential for a significant protein-CNT binding

- Protein-binding is dependent on the three-dimensional arrangement of the carbon atoms of the CNTs 
Table 2. Key literature (for the past two decades) concerning the carbon nanotubes (CNT)-protein interaction. Please note that this table is not a complete list, however highlights the key papers regarding CNT-biomolecule research.

\begin{tabular}{|c|c|c|c|c|}
\hline $\begin{array}{l}\text { Year of } \\
\text { Publication }\end{array}$ & $\begin{array}{l}\text { Type of } \\
\text { CNT }\end{array}$ & $\begin{array}{l}\text { Type of Adsorbed } \\
\text { Protein(s) }\end{array}$ & Major Outcome & Reference \\
\hline \multirow{2}{*}{2001} & \multirow{2}{*}{ SWCNT } & $\begin{array}{l}\text { Ferritin, } \\
\text { streptavidin }\end{array}$ & $\begin{array}{l}\text { Proteins with primary and secondary amines } \\
\text { adsorbed onto f-SWCNT via } \pi-\pi \\
\text { stacking interactions. }\end{array}$ & [82] \\
\hline & & $\begin{array}{l}\text { Proteins rich in } \\
\text { surface amines } \\
\text { (antibody for C60) }\end{array}$ & $\begin{array}{l}\text { SWCNT with a curved hydrophobic } \\
\pi \text {-electron-rich surface bound on the } \\
\text { hydrophobic binding sites of proteins. }\end{array}$ & {$[54]$} \\
\hline \multirow[b]{2}{*}{2002} & \multirow[b]{2}{*}{ SWCNT } & $\begin{array}{l}\text { Metalloproteins, } \\
\text { Enzymes }\end{array}$ & $\begin{array}{l}\text { Enzymes immobilized on SWCNTs retain } \\
\text { their catalytic activity. }\end{array}$ & [68] \\
\hline & & $\begin{array}{l}\text { Streptavidin } \\
\text { (various proteins) }\end{array}$ & $\begin{array}{l}\text { Pre-coating of SWCNTs with triton X-100 } \\
\text { prior PEG coating prevented the adsorption } \\
\text { of small proteins onto SWCNT } \\
\text { nearly completely. }\end{array}$ & [78] \\
\hline \multirow{2}{*}{2003} & SWCNT & $\begin{array}{l}\text { Amphiphilic } \\
\alpha \text {-helical peptide }\end{array}$ & $\begin{array}{l}\text { The apolar residues of amphiphilic proteins } \\
\text { bound to the surface of SWCNT and the } \\
\text { polar residues of the proteins were located } \\
\text { against the solvent face. }\end{array}$ & {$[55]$} \\
\hline & MWCNT & $\begin{array}{l}\text { Phage and other } \\
\text { types of peptides }\end{array}$ & $\begin{array}{l}\text { Peptides that were rich in histidine and } \\
\text { tryptophan bound at special locations of the } \\
\text { MWCNTs by hydrophobic interactions. }\end{array}$ & {$[62]$} \\
\hline \multirow{4}{*}{2004} & \multirow{3}{*}{ SWCNT } & $\begin{array}{l}\alpha \text {-Chymotrypsin, } \\
\text { soybean } \\
\text { peroxidase }\end{array}$ & $\begin{array}{l}\text { The enzymes changed their secondary } \\
\text { structures upon adsorption onto the } \\
\text { SWCNTs, which caused a decrease or nearly } \\
\text { complete loss of their activity. }\end{array}$ & {$[66]$} \\
\hline & & Ferritin & $\begin{array}{l}\text { A covalent coating of SWCNT with PEG was } \\
\text { alleviating or even completely eliminating } \\
\text { the natural protein affinity of the SWCNTs. }\end{array}$ & [77] \\
\hline & & $\begin{array}{l}\text { Amphiphilic } \\
\alpha \text {-helical peptide }\end{array}$ & $\begin{array}{l}\text { The binding of polar residues of amphiphilic } \\
\text { proteins onto the surface of SWCNTs } \\
\text { increased the dispersion of the } \\
\text { SWCNTs in water. }\end{array}$ & {$[56]$} \\
\hline & $\mathrm{CNT}$ & Streptavidin & $\begin{array}{l}\text { Protein adsorption onto CNTs occurred } \\
\text { through interactions between the amine } \\
\text { groups of the protein and the hydrophobic } \\
\text { surface of the CNTs. }\end{array}$ & [83] \\
\hline \multirow{2}{*}{2005} & \multirow{2}{*}{ SWCNT } & $\begin{array}{l}\text { Amphiphilic } \\
\alpha \text {-helical peptide }\end{array}$ & $\begin{array}{l}\text { Amphiphilic peptides bound non-covalently } \\
\text { with their apolar residues onto the SWCNTs, } \\
\text { which resulted in a better solubilisation } \\
\text { of the SWCNTs. }\end{array}$ & {$[51]$} \\
\hline & & $\begin{array}{l}\text { Amphiphilic } \\
\text { peptide helix } \\
\text { (nano-1) }\end{array}$ & $\begin{array}{l}\text { The aromatic residues of the peptides } \\
\text { interacted with the SWCNT surface, } \\
\text { which was leading to a better dispersion } \\
\text { of the SWCNTs. }\end{array}$ & {$[52]$} \\
\hline
\end{tabular}


Table 2. Cont.

\begin{tabular}{|c|c|c|c|c|}
\hline $\begin{array}{l}\text { Year of } \\
\text { Publication }\end{array}$ & Type of CNT & $\begin{array}{l}\text { Type of Adsorbed } \\
\text { Protein(s) }\end{array}$ & Major Outcome & Reference \\
\hline \multirow{6}{*}{2006} & \multirow{6}{*}{ SWCNT } & Model proteins & $\begin{array}{l}\text { Protein coated SWCNTs were incorporated } \\
\text { by the cells via energy dependent } \\
\text { endocytosis through clathrin-coated pits. }\end{array}$ & {$[84]$} \\
\hline & & $\begin{array}{l}\text { Different types of } \\
\text { proteins }\end{array}$ & $\begin{array}{l}\text { Proteins adsorbed onto SWCNTs via } \pi-\pi \\
\text { stacking as well as amine interactions, } \\
\text { whereas the hydrophilic protein moieties } \\
\text { were located towards the water face. }\end{array}$ & {$[57]$} \\
\hline & & $\begin{array}{l}\text { Polyline, } \\
\text { polytryptophan }\end{array}$ & $\begin{array}{l}\text { A strong adhesive force was registrated } \\
\text { between the protonated amine-groups of the } \\
\text { protein (polylysine) and the carboxyl-groups } \\
\text { of the oxidized CNTs. }\end{array}$ & {$[58]$} \\
\hline & & Lysozyme & $\begin{array}{l}\pi-\pi \text { stacking and hydrophobic interactions as } \\
\text { well as protonated amine interactions } \\
\text { between proteins and SWCNT were } \\
\text { responsible for the dispersion } \\
\text { of the SWCNTs. }\end{array}$ & [49] \\
\hline & & $\begin{array}{l}\text { Fibrinogen, } \\
\text { apolipoproteins } \\
\text { (AI, AIV, CIII) }\end{array}$ & $\begin{array}{l}\text { Protein binding onto SWCNT was } \\
\text { highly selective. }\end{array}$ & {$[45]$} \\
\hline & & $\begin{array}{l}\text { Peptides from } \\
\text { phage libraries }\end{array}$ & $\begin{array}{l}\text { Hydrophobic as well as } \pi-\pi \text { interactions } \\
\text { between proteins and SWCNTs were } \\
\text { important for a selective protein } \\
\text { binding onto SWCNTs. }\end{array}$ & [46] \\
\hline \multirow{4}{*}{2007} & \multirow{3}{*}{ SWCNT } & $\begin{array}{l}\text { Foetal bovine } \\
\text { plasma, human } \\
\text { serum/plasma } \\
\text { protein }\end{array}$ & $\begin{array}{l}\text { The uptake of SWCNT occurred by } \\
\text { pathways associated with the adsorbed } \\
\text { proteins. The proteins modulated in addition } \\
\text { the toxicity of the SWCNTs. }\end{array}$ & [43] \\
\hline & & $\begin{array}{l}\text { Different types } \\
\text { of proteins }\end{array}$ & $\begin{array}{l}\text { The primary, secondary and tertiary } \\
\text { structures of proteins and the } \mathrm{pH} \text { of the } \\
\text { dispersion medium were important to obtain } \\
\text { a high yield of de-bundeled CNTs }\end{array}$ & [61]. \\
\hline & & $\begin{array}{l}\text { Different types } \\
\text { of peptides }\end{array}$ & $\begin{array}{l}\text { Disulfide bonds adsorbed onto the SWCNTs } \\
\text { and by that they solubilize the SWCNTs } \\
\text { without altering their electronic structure. }\end{array}$ & {$[60]$} \\
\hline & DWCNT & $\begin{array}{l}\text { Surfactant proteins } \\
\text { A and D }\end{array}$ & $\begin{array}{l}\text { Supernatant protein A and D adsorbed } \\
\text { selectively onto DWCNTs out of different } \\
\text { pulmonary surfactant protein samples. }\end{array}$ & [59] \\
\hline \multirow{4}{*}{2008} & $\begin{array}{l}\text { SWCNT, } \\
\text { MWCNT }\end{array}$ & Ribonuclease A & $\begin{array}{l}\text { CNTs functionalized with carboxylic groups } \\
\text { interacted with the enzyme and caused } \\
\text { a reduction of its activity by changing } \\
\text { its conformation. }\end{array}$ & {$[64]$} \\
\hline & \multirow[t]{2}{*}{$\begin{array}{l}\text { MWCNT, } \\
\text { f-MWCNT }\end{array}$} & $\begin{array}{l}\text { Bovine serum } \\
\text { albumin (BSA) and } \\
\text { different types of } \\
\text { proteins }\end{array}$ & $\begin{array}{l}\text { Electrostatic and stereo-chemical properties } \\
\text { of the MWCNTs and the proteins as well as } \\
\text { the curvature of the MWCNTs were affecting } \\
\text { the protein binding affinity onto } \\
\text { the MWCNTs. }\end{array}$ & {$[81]$} \\
\hline & & $\begin{array}{l}\text { Human plasma } \\
\text { and serum proteins }\end{array}$ & $\begin{array}{l}\text { Functionalization of the MWCNTs affected } \\
\text { the patterns of adsorbed proteins onto the } \\
\text { MWCNT, which resulted in a better } \\
\text { biocompatibility of the MWCNTs. }\end{array}$ & [76] \\
\hline & CNT & $\begin{array}{l}\text { A-sub-domain of } \\
\text { human serum } \\
\text { albumin }\end{array}$ & $\begin{array}{l}\text { The adsorption of proteins onto CNTs caused } \\
\text { a conformation change of the secondary } \\
\text { protein structure, which resulted in a } \\
\text { decrease of the protein activity. }\end{array}$ & [67] \\
\hline
\end{tabular}


Table 2. Cont.

\begin{tabular}{|c|c|c|c|c|}
\hline $\begin{array}{l}\text { Year of } \\
\text { Publication }\end{array}$ & Type of CNT & $\begin{array}{l}\text { Type of Adsorbed } \\
\text { Protein(s) }\end{array}$ & Major Outcome & Reference \\
\hline 2009 & $\begin{array}{l}\text { MWCNT, } \\
\text { f-MWCNT }\end{array}$ & $\alpha$-Chymotrypsin & $\begin{array}{l}\text { Enzymes bound onto MWCNTs through } \pi-\pi \\
\text { stacking and hydrophobic interactions, } \\
\text { which resulted in a competitive inhibition of } \\
\text { the enzyme activity. }\end{array}$ & [65] \\
\hline \multirow{3}{*}{2010} & \multirow[b]{2}{*}{ SWCNT } & Model surfactant & $\begin{array}{l}\text { Surfactants with a larger hydrophilic head } \\
\text { group was leading to a significant better } \\
\text { dispersion stability of SWCNTs. }\end{array}$ & {$[75]$} \\
\hline & & Model protein & $\begin{array}{l}\text { Hydrophobic interactions between the } \\
\text { hydrophobic core of the proteins and the } \\
\text { SWCNTs formed stable complexes, which } \\
\text { caused a blockage of the active sides of } \\
\text { the proteins. }\end{array}$ & {$[63]$} \\
\hline & $\begin{array}{l}\text { MWCNT, } \\
\text { f-MWCNT }\end{array}$ & $\begin{array}{l}\text { Pulmonary } \\
\text { surfactant } \\
\left(\text { Curosurf }^{\circledR}\right)\end{array}$ & $\begin{array}{l}\text { The pre-coating of MWCNTs with a lung } \\
\text { surfactant influenced the protein binding } \\
\text { onto the MWCNTs and resulted in } \\
\text { characteristic binding patterns. }\end{array}$ & {$[70]$} \\
\hline \multirow[t]{2}{*}{2011} & $\begin{array}{l}\text { SWCNT, } \\
\text { MWCNT }\end{array}$ & Serum proteins & $\begin{array}{l}\text { The adsorption capacity of CNTs for proteins } \\
\text { was dependent on the type, arrangement } \\
\text { model, size and surface modification } \\
\text { of the CNTs. }\end{array}$ & {$[42]$} \\
\hline & SWCNT & $\begin{array}{l}\text { Human } \\
\text { serum proteins }\end{array}$ & $\begin{array}{l}\text { Competitive binding of blood proteins onto } \\
\text { the SWCNT surface can alter the cellular } \\
\text { interaction pathways, resulting in } \\
\text { a reduced cytotoxicity. }\end{array}$ & {$[44]$} \\
\hline \multirow{3}{*}{2011} & SWCNT & $\begin{array}{l}\text { Bovine serum } \\
\text { albumin (BSA) }\end{array}$ & $\begin{array}{l}\text { Bovine serum albumin dispersed SWCNTs } \\
\text { readily entered into the cells and did not } \\
\text { acute deleterious cellular effects. }\end{array}$ & {$[73]$} \\
\hline & $\begin{array}{l}\text { SWCNT, } \\
\text { DWCNT }\end{array}$ & Serum proteins & $\begin{array}{l}\text { The adsorption of enzymes of the immune } \\
\text { system to the hydrophobic SWCNT surface } \\
\text { didn't caused an activation of the enzymes. }\end{array}$ & {$[85]$} \\
\hline & MWCNT & Blood proteins & $\begin{array}{l}\text { A surface modification of the MWCNT } \\
\text { affected their patterns of adsorbed proteins, } \\
\text { which resulted in a modification of the } \\
\text { biocompatibility of the MWCNTs. }\end{array}$ & {$[48]$} \\
\hline \multirow{3}{*}{2012} & \multirow{2}{*}{ SWCNT } & $\begin{array}{l}\text { Bovine serum } \\
\text { albumin (BSA) }\end{array}$ & $\begin{array}{l}\text { Bovine serum albumin coated SWCNTs were } \\
\text { taken up by the cells within seconds. } \\
\text { However, the cells were able to expel the } \\
\text { incorporated BSA-SWCNT complexes over } \\
\text { hours and days. }\end{array}$ & {$[72]$} \\
\hline & & $\begin{array}{l}\text { Different types } \\
\text { of proteins }\end{array}$ & $\begin{array}{l}\text { The stability of a SWCNT-protein complex } \\
\text { had a substantial influence on the cellular } \\
\text { uptake and the uptake of a certain protein } \\
\text { was dependent from the cell type. }\end{array}$ & {$[86]$} \\
\hline & MWCNT & $\begin{array}{l}\text { Pulmonary } \\
\text { surfactant } \\
\text { (Curosurf) }\end{array}$ & $\begin{array}{l}\text { The pre-coating of MWCNTs with a lung } \\
\text { surfactant affected the uptake of the } \\
\text { MWCNTs without significantly altering the } \\
\text { cytotoxicity of the MWCNTs. }\end{array}$ & {$[71]$} \\
\hline
\end{tabular}


Table 2. Cont.

\begin{tabular}{|c|c|c|c|c|}
\hline $\begin{array}{l}\text { Year of } \\
\text { Publication }\end{array}$ & Type of CNT & $\begin{array}{l}\text { Type of Adsorbed } \\
\text { Protein(s) }\end{array}$ & Major Outcome & Reference \\
\hline \multirow[t]{4}{*}{2013} & MWCNT & $\begin{array}{l}\text { Human cellular } \\
\text { proteins (HeLa } \\
\text { cells lysate) }\end{array}$ & $\begin{array}{l}\text { Electrostatic, stereochemical properties, } \\
\text { diameter and curvature of the MWCNTs } \\
\text { were significantly affecting the adsorption of } \\
\text { proteins onto the MWCNTs. }\end{array}$ & [79] \\
\hline & SWCNT & Plasma proteins & $\begin{array}{l}\text { The surface PEG conformation of } \\
\text { SWCNT-PEG complexes affected the pattern } \\
\text { of adsorbed plasma proteins onto the } \\
\text { SWCNTs and influenced the biodistribution } \\
\text { of the SWCNT-PEG complexes. }\end{array}$ & {$[53]$} \\
\hline & $\begin{array}{l}\text { SWCNT, } \\
\text { f-SWCNT, } \\
\text { MWCNT, } \\
\text { f-MWCNT }\end{array}$ & $\begin{array}{l}\text { Foetal Bovine } \\
\text { serum (FBS) }\end{array}$ & $\begin{array}{l}\text { Functionalized CNTs were able to bind a } \\
\text { number of unique proteins, which implied } \\
\text { that electrostatic interactions and specific } \\
\text { covalent bonding were involved. }\end{array}$ & {$[80]$} \\
\hline & CNT & $\begin{array}{l}\text { Different types } \\
\text { of proteins }\end{array}$ & $\begin{array}{l}\pi-\pi \text { stacking and hydrophobic interactions } \\
\text { were responsible for the adsorption of } \\
\text { proteins onto CNTs. The protein adsorption } \\
\text { leaded to a reduction of the cytotoxicity and } \\
\text { to a loss of the enzymatic activity } \\
\text { of the proteins. }\end{array}$ & [47] \\
\hline
\end{tabular}

\section{Discussion}

According to the fiber paradigm, in addition to the geometry of fibers, their bio-persistence is the most important characteristic determining their biological impact towards the lungs. It has been shown that asbestos fibers, as well as long and thick MWCNT, can follow the fiber paradigm. As these fibers are made of different materials, the composition of the fibers is not considered as one of the most relevant factors, but in fact influences their persistence within the human body and the biological effects. The importance of a bio-corona that is formed around a nanomaterial entering a biological environment is undoubted and a view on the recent literature on fiber-protein interactions, as summarized here, shows the importance of the protein corona on the fiber toxicity. Thus, the question is "how does the protein corona influence the fiber toxicity?". Are there characteristics of the protein corona bound to (nano)fibers that differ from those obtained by other shapes? Is there anything that can be described as a fiber-specific effect (associated with the bound proteins)?

The majority of studies concerning the composition of the protein corona were performed on nanomaterials of similar shape but different size or surface characteristics. To answer the question, if there are certain fiber-specific characteristics within the protein-corona of fibers, we compared the "top-ten" or "hit list" of the proteins found on fibers and other nanomaterials of different shapes and materials: i.e., CNTs, silica nanoparticles [87,88], gold nanoparticles [89-91], polymeric particles $[27,88,92]$, and iron oxide nanoparticles [93,94]. Despite the differences 
in morphology (shape and size), surface characteristics and material composition, several proteins (e.g., albumin, apolipoprotein $\mathrm{AI}$ and $\mathrm{E}$, fibrinogen, fibronectin, vitronectin, complement factor $\mathrm{C} 3, \alpha 2$-macroglobulin) were found on nearly all protein coronas investigated. This leads to additional questions, regarding our knowledge on the protein corona, the methods applied to investigate the detailed composition of the protein corona as well as the comparability of the results obtained by different approaches.

\subsection{Comparison of Protein Coronas}

Comparison of the composition of protein coronas of different nanomaterials can be triggered by several factors. First, the experimental protocols applied can have a significant impact on the precision, reproducibility and comparability of the studies. Thus, the variability between protein coronas of identical materials but obtained by different protocols might be given.

Second, the protein corona formation is a very complex and dynamic process. Within less than few seconds after exposition of the nanomaterials to a biological medium, the protein corona is formed [88]. The composition of the protein corona can also vary over time. High abundant serum proteins can dominate adsorption on the nanomaterial surface at short incubation times, but with time they can be replaced by other proteins with lower abundance but higher affinity [2]. Testing strategies involving separation methods are not able to enclose the dynamic processes taking place at the protein-nanomaterial interface. Thus, the incubation time of the nanomaterial in the bio-fluid has an effect on the observed protein corona. Even if those studies are performed time-dependently, they only give us a semi-dynamic picture. Thus, the variation of the composition of the protein corona at different time points might be quite high, perhaps in the same range as the variation between different materials investigated or protocols being applied.

One factor that can further blur the picture obtained from the protein corona is the fact that the protein profile of nanomaterials is not necessarily the protein pattern found on each and every particle in the dispersion, but rather the sum of proteins bound to the sum of available surface area within the nanomaterial dispersion. This can be easily illustrated when the diversity of proteins found adsorbed to a particle dispersion is compared to the available surface area of a single particle, the particle size and the size of protein molecules detected in the protein corona. Tenzer et al. [88] detected almost up to 300 different proteins within the protein adsorption pattern of silica and polystyrene nanoparticle samples. They investigated the protein corona of silica nanoparticles of two different sizes, as well as those of polystyrene nanoparticles with different surface functionalization, formed after exposure to human plasma [88].

As the adsorption pattern on material surfaces are dependent on the morphology and physicochemical properties of the nanomaterial, slight variations within a sample 
might also influence the resulting protein pattern. The measured physicochemical properties of nanomaterial dispersions are an average of the properties of all particles within the sample. In other words, the sum of the available particle surface cannot necessarily be regarded as a single and homogeneous surface area cut into equal pieces. The protein adsorption pattern of one particle might differ from the pattern adsorbed on another particle within the same sample. It is important to minimize the variability of fibers or particles within a sample to obtain results that can be linked to certain material properties. Therefore, the number of different proteins detected within the protein corona should be interpreted with care. A high number of different protein types within a protein profile does not necessarily reflect a higher precision of the methodology and might instead derive from a certain variability of physicochemical parameters in a sample.

In summary, the comparison of different studies is complicated by several factors and it was not possible to find any characteristic of the protein corona that could be clearly linked, specifically to fiber morphology alone.

\subsection{Methodology and Challenges}

The protein corona is often divided into a hard corona, which is composed of tightly bound proteins, and a soft corona, a layer of proteins which is subject to rapid exchange of its components. Monopoli et al. [2] suggest that the interface between the hard and soft corona is the key factor determining the biological effects of the nanomaterial. The residence time of biomolecules in the protein corona is seen as one of the most important determinants.

In other words, the interaction of proteins bound to the particle surface and those which bind to the adsorbed proteins is of high interest. This leads to the question if the recently applied methods to investigate the composition of the protein corona enable us to study such interactions. As described before, the methods recently applied are based on a separation of the tightly bound proteins from loosely bound protein fraction. As a consequence, these methods can only consider proteins tightly bound to the nanomaterial or nanomaterial-protein complexes. The obtained results do not allow us to determine if all proteins found within the corona are directly bound to the nanomaterials surface or whether their association with the nanomaterial is mediated by other proteins already bound to the surface. Information as to the conformational state of the bound proteins, as well as regarding the consequences of their binding to the nanomaterial for their biological function is, at best, limited. As mentioned above, the applied methods do not provide a resolution of the dynamic processes taking place at the solid-liquid interface. Without question, much progress has been performed in the investigation of the composition of the protein corona of nanomaterials, but extension of our recent tool-box is needed to gain further in-sight in the underlying mechanisms of the complex interaction of proteins at 
the solid-liquid interface and to correlate nanomaterial properties to the corona properties and resulting biological effects.

\subsection{Influence of Shape on Nanomaterial-Protein Interaction}

It is well accepted that several nanomaterial characteristics as size, material composition, and surface functionalization, determine the protein adsorption pattern to these materials in biological media. The influence of the shape of the nanomaterials, especially the difference between fibrous and spherical nanomaterials of similar elemental composition, is not well-understood. But in fact, there is evidence that the shape and morphology of the materials has a significant influence on the protein adsorption. In addition, it has been noticed that other factors, such as the atomic-scale surface topography, impurities, and structural defects, can have a significant impact on the protein adsorption to nanomaterials.

Recently, Gagner et al. [95,96] demonstrated that not only the size of a nanomaterial, but also its shape and crystal structure can have a significant effect on the nanomaterial protein adsorption pattern in biological fluids. In a first study, they systematically varied the morphology of gold nanomaterials and compared the binding of lysozyme and a-chymotrypsin to gold nanospheres and nanorods of comparable diameter. For both proteins, they observed differences in surface coverage dependent on the morphology of the nanomaterials. At normalized surface area, the amount of proteins bound to nanorods was higher than to nanospheres. Due to their cylindrical morphology, nanorods exhibited a relatively flat surface along the axis. This is thought to facilitate protein binding in contrast to the nanosphere surface, which is highly curved in all directions. In addition, enzyme activity was more preserved on gold nanospheres than on gold nanorods. These results are in line with reports that enzyme stability is higher on surfaces with high curvature than on those with more flat surface, demonstrated with silica [97], and gold nanoparticles [98], as well as by comparison of carbon nanotubes and graphite [99].

Comparison of the protein pattern found on titanium dioxide spheres, nanorods and nanotubes, by Deng et al. [100] showed that the shape of titanium dioxide nanomaterials can have an influence on the protein composition of the corona. Protein profiles obtained by 2D gel electrophoresis showed that spherical titanium dioxide nanoparticles $(\varnothing \sim 21 \mathrm{~nm})$ adsorbed qualitatively more proteins than titanium dioxide nanorods $(\varnothing \sim 27 \mathrm{~nm}$ ) or titanium dioxide nanotubes $(\varnothing \sim 9 \mathrm{~nm})$.

Taken together, there are several hints that the morphology of a material has an impact on the protein binding to its surface. For several materials, such as silica, titanium dioxide, gold, and carbon, nano-objects with different shapes are available. However, how the shape influences the protein adsorption pattern in a complex medium can only be deduced, due to the lack of systematic studies focused on this parameter. By a view on the available literature, clear fiber-specific characteristics 
of the protein-adsorption pattern within a complex medium could not be identified, but in fact, it could be shown that the protein corona has a significant impact on the biological effects of fiber materials (i.e., asbestos).

Acknowledgments: The authors would like to acknowledge the Swiss National Foundation, the Swiss National Research Programme 64, CCMX Matlife through the project CCMX-VIGO (Contract N 350601), an Empa internal grant and the Adolphe Merkle Foundation for their financial support.

Author Contributions: Peter Wick, Barbara Rothen-Rutishauser and Alke Petri-Fink elaborated the idea, structure and concept of the review. Melanie Kucki, Jean-Pierre Kaiser and Martin J. D. Clift performed the literature research \& analysis, as well as wrote and prepared the manuscript. All authors read, commented on, and improved the manuscript. All authors are entirely responsible for the content of the manuscript.

Conflicts of Interest: The authors declare no conflict of interest.

\section{References}

1. Casals, E.; Puntes, V.F. Inorganic nanoparticle biomolecular corona: Formation, evolution and biological impact. Nanomedicine (Lond) 2012, 7, 1917-1930.

2. Monopoli, M.P.; Aberg, C.; Salvati, A.; Dawson, K.A. Biomolecular coronas provide the biological identity of nanosized materials. Nat. Nanotechnol. 2012, 7, 779-786.

3. Aggarwal, P.; Hall, J.B.; McLeland, C.B.; Dobrovolskaia, M.A.; McNeil, S.E. Nanoparticle interaction with plasma proteins as it relates to particle biodistribution, biocompatibility and therapeutic efficacy. Adv. Drug Deliv. Rev. 2009, 61, 428-437.

4. Iijima, S. Helical microtubules of graphitic carbon. Nature 1991, 354, 56-58.

5. Donaldson, K.; Murphy, F.A.; Duffin, R.; Poland, C.A. Asbestos, carbon nanotubes and the pleural mesothelium: A review of the hypothesis regarding the role of long fibre retention in the parietal pleura, inflammation and mesothelioma. Part. Fibre Toxicol. 2010, 7, 1-17.

6. Dogan, A.U.; Dogan, M.; Hoskins, J.A. Erionite series minerals: Mineralogical and carcinogenic properties. Environ. Geochem. Health 2008, 30, 367-381.

7. Carbone, M.; Baris, Y.I.; Bertino, P.; Brass, B.; Comertpay, S.; Dogan, A.U.; Gaudino, G.; Jube, S.; Kanodia, S.; Partridge, C.R.; et al. Erionite exposure in North Dakota and Turkish villages with mesothelioma. Proc. Natl. Acad. Sci. USA 2011, 108, 13618-13623.

8. Faller, A.; Schünke, M. Der Körper des Menschen; Thieme: Stuttgart, Germany, 1999. (in German)

9. Gil, J.; Bachofen, H.; Gehr, P.; Weibel, E.R. Alveolar volume-surface area relation in airand saline-filled lungs fixed by vascular perfusion. J. Appl. Physiol. 1979, 47, 990-1001.

10. Gehr, P.; Bachofen, M.; Weibel, E.R. The normal human lung: Ultrastructure and morphometric estimation of diffusion capacity. Respir. Physiol. 1978, 32, 121-140.

11. Ochs, M.; Weibel, E.R. Functional design of the human lung for gas exchange. In Fishman's Pulmonary Diseases and Disorders; Fishman, A.P., Elias, J., Fishman, J., Grippi, M., Senior, R., Eds.; McGraw-Hill Professional: New York, NY, USA, 2008; pp. 23-70. 
12. Lohmann-Matthes, M.L.; Steinmüller, C.; Franke-Ullmann, G. Pulmonary macrophages. Eur. Respir. J. 1994, 7, 1678-1689.

13. Holt, P.G.; Stumbles, P.A. Characterization of dendritic cell populations in the respiratory tract. J. Aerosol. Med. 2000, 13, 361-367.

14. Donaldson, K.; Seaton, A. A short history of the toxicology of inhaled particles. Part. Fibre Toxicol. 2012, 9, 13.

15. Brown, D.M.; Kinloch, I.A.; Bangert, U.; Windleb, A.H.; Walterd, D.M.; Walkerd, G.S.; Scotchfordd, C.A.; Donaldsone, K.; Stonea, V. An in vitro study of the potential of carbon nanotubes and nanofibres to induce inflammatory mediators and frustrated phagocytosis. Carbon 2007, 45, 1743-1756.

16. Oberdorster, G. Toxicokinetics and effects of fibrous and nonfibrous particles. Inhal. Toxicol. 2002, 14, 29-56.

17. Liu, W.; Rose, J.; Plantevin, S.; Auffan, M.; Bottero, J.Y.; Vidaud, C. Protein corona formation for nanomaterials and proteins of a similar size: Hard or soft corona? Nanoscale 2013, 5, 1658-1668.

18. Wick, P.; Clift, M.J.; Rösslein, M.; Rothen-Rutishauser, B. A brief summary of carbon nanotubes science and technology: A health and safety perspective. ChemSusChem 2011, 4, 905-911.

19. Poland, C.A.; Duffin, R.; Kinloch, I.; Maynard, A.; Wallace, W.A.; Seaton, A.; Stone, V.; Brown, S.; Macnee, W.; Donaldson, K. Carbon nanotubes introduced into the abdominal cavity of mice show asbestos-like pathogenicity in a pilot study. Nat. Nanotechnol. 2008, 3, 423-428.

20. Donaldson, K.; Tran, C.L. An introduction to the short-term toxicology of respirable industrial fibres. Mutat. Res. 2004, 553, 5-9.

21. Miller, B.G.; Jones, A.D.; Searl, A.; Buchanan, D.; Cullen, R.T.; Soutar, C.A.; Davis, J.M.; Donaldson, K. Influence of characteristics of inhaled fibres on development of tumours in the rat lung. Ann. Occup. Hyg. 1999, 43, 167-179.

22. Stanton, M.F.; Wrench, C. Mechanisms of mesothelioma induction with asbestos and fibrous glass. J. Natl. Cancer Inst. 1972, 48, 797-821.

23. Kamstrup, O.; Ellehauge, A.; Chevalier, J.; Davis, J.M.; McConnell, E.E.; Thévenaz, P. Chronic inhalation studies of two types of stone wool fibers in rats. Inhal. Toxicol. 2001, 13, 603-621.

24. UmweltWissen—Produkte \& Abfall: Künstliche Mineralfasern. 2013. Available online: http://www.lfu.bayern.de/umweltwissen/doc/uw_32_kuenstliche_mineralfasern. pdf (accessed on 19 June 2014).

25. Jaurand, M.C.; Renier, A.; Daubriac, J. Mesothelioma: Do asbestos and carbon nanotubes pose the same health risk? Part. Fibre Toxicol. 2009, 6, 16.

26. Lynch, I.; Dawson, K.A. Protein-nanoparticle interactions. Nano Today 2008, 3, 40-47.

27. Sempf, K.; Arrey, T.; Gelperina, S.; Schorge, T.; Meyer, B.; Karas, M.; Kreuter, J. Adsorption of plasma proteins on uncoated PLGA nanoparticles. Eur. J. Pharm. Biopharm. 2013, $85,53-60$. 
28. Small, I.; Peeters, N.; Legeai, F.; Lurin, C. Predotar: A tool for rapidly screening proteomes for N-terminal targeting sequences. Proteomics 2004, 4, 1581-1590.

29. Cedervall, T.; Lynch, I.; Foy, M.; Berggård, T.; Donnelly, S.C.; Cagney, G.; Linse, S.; Dawson, K.A. Detailed identification of plasma proteins adsorbed on copolymer nanoparticles. Angew. Chem. 2007, 119, 5856-5858.

30. Cedervall, T.; Lynch, I.; Lindman, S.; Berggård, T.; Thulin, E.; Nilsson, H.; Dawson, K.A.; Linse, S. Understanding the nanoparticle-protein corona using methods to quantify exchange rates and affinities of proteins for nanoparticles. Proc. Natl. Acad. Sci. USA 2007, 104, 2050-2055.

31. Morgan, A. Absorption of human serum albumin by asbestiform minerals and its application to the measurement of surface areas of dispersed samples of chrysotile. Environ. Res. 1974, 7, 330-341.

32. Light, W.G.; Wei, E.T. Surface charge and asbestos toxicity. Nature 1977, 265, 537-539.

33. Morgan, A.; Davies, P.; Wagner, J.C.; Berry, G.; Holmes, A. The biological effects of magnesium-leached chrysotile asbestos. Br. J. Exp. Pathol. 1977, 58, 465-473.

34. Valerio, F.; Veggi, M.; Santi, L. Adsorption isotherms of albumin and ferritin on rhodesian chrysotile. Environ. Res. 1980, 21, 186-189.

35. Valerio, F.; Balducci, D.; Scarabelli, L. Selective adsorption of serum proteins by chrysotile and crocidolite. Environ. Res. 1986, 41, 432-439.

36. Valerio, F.; Balducci, D.; Lazzarotto, A. Adsorption of proteins by chrysotile and crocidolite: role of molecular weight and charge density. Environ. Res. 1987, 44, 312-320.

37. Kamp, D.W.; Dunne, M.; Anderson, J.A.; Weitzman, S.A.; Dunn, M.M. Serum promotes asbestos-induced injury to human pulmonary epithelial cells. J. Lab. Clin. Med. 1990, 116, 289-297.

38. Scheule, R.K.; Holian, A. Modification of asbestos bioactivity for the alveolar macrophage by selective protein adsorption. Am. J. Respir. Cell Mol. Biol. 1990, 2, 441-448.

39. Boylan, A.M.; Sanan, D.A.; Sheppard, D.; Broaddus, V.C. Vitronectin enhances internalization of crocidolite asbestos by rabbit pleural mesothelial cells via the integrin alpha v beta 5. J. Clin. Invest. 1995, 96, 1987-2001.

40. Liu, W.; Ernst, J.D.; Broaddus, V.C. Phagocytosis of crocidolite asbestos induces oxidative stress, DNA damage, and apoptosis in mesothelial cells. Am. J. Respir. Cell Mol. Biol. 2000, 23, 371-378.

41. Wu, J.; Liu, W.; Koenig, K.; Idell, S.; Broaddus, V.C. Vitronectin adsorption to chrysotile asbestos increases fiber phagocytosis and toxicity for mesothelial cells. Am. J. Physiol. Lung Cell. Mol. Physiol. 2000, 279, L916-L923.

42. Du, J.; Ge, C.; Liu, Y.; Bai, R.; Li, D.; Yang, Y.; Liao, L.; Chen, C. The interaction of serum proteins with carbon nanotubes depend on the physicochemical properties of nanotubes. J. Nanosci. Nanotechnol. 2011, 11, 10102-10110.

43. Dutta, D.; Sundaram, S.K.; Teeguarden, J.G.; Riley, B.J.; Fifield, L.S.; Jacobs, J.M.; Addleman, S.R.; Kaysen, G.A.; Moudgil, B.M.; Weber, T.J. Adsorbed proteins influence the biological activity and molecular targeting of nanomaterials. Toxicol. Sci. 2007, 100, 303-315. 
44. Ge, C.; Du, J.; Zhao, L.; Wang, L.; Liu, Y.; Li, D.; Yang, Y.; Zhou, R.; Zhao, Y.; Chai, Z.; et al. Binding of blood proteins to carbon nanotubes reduces cytotoxicity. PNAS 2011, 108, 16968-16973.

45. Salvador-Morales, C.; Flahaut, E.; Sim, E.; Sloan, J.; Green, M.L.; Sim, R.B. Complement activation and protein adsorption by carbon nanotubes. Mol. Immunol. 2006, 43, 193-201.

46. Su, Z.; Leung, T.; Honek, J.F. Conformational selectivity of peptides for single-walled carbon nanotubes. J. Phys. Chem. B 2006, 110, 23623-23627.

47. Zuo, G.; Kang, S.G.; Xiu, P.; Zhao, Y.; Zhou, R. Interactions between proteins and carbon-based nanoparticles: Exploring the origin of nanotoxicity at the molecular level. Small 2013, 9, 1546-1556.

48. Yan, L.; Zhao, F.; Li, S.; Hu, Z.; Zhao, Y. Low-toxic and safe nanomaterials by surface-chemical design, carbon nanotubes, fullerenes, metallofullerenes, and graphenes. Nanoscale 2011, 3, 362-382.

49. Nepal, D.; Geckeler, K.E. pH-sensitive dispersion and debundling of single-walled carbon nanotubes: Lysozyme as a tool. Small 2006, 2, 406-412.

50. Ortiz-Acevedo, A.; Xie, H.; Zorbas, V.; Sampson, W.M.; Dalton, A.B.; Baughman, R.H.; Draper, R.K.; Musselman, I.H.; Dieckmann, G.R. Diameter-selective solubilization of single-walled carbon nanotubes by reversible cyclic peptides. J. Am. Chem. Soc. 2005, 127, 9512-9517.

51. Zorbas, V.; Smith, A.L.; Xie, H.; Ortiz-Acevedo, A.; Dalton, A.B.; Dieckmann, G.R.; Draper, R.K.; Baughman, R.H.; Musselman, I.H. Importance of aromatic content for peptide/single-walled carbon nanotube interactions. J. Am. Chem. Soc. 2005, 127, 12323-12328.

52. Wick, P.; Manser, P.; Limbach, L.K.; Dettlaff-Weglikowska, U.; Krumeich, F.; Roth, S.; Stark, W.J.; Bruinink, A. The degree and kind of agglomeration affect carbon nanotube cytotoxicity. Toxicol. Lett. 2007, 168, 121-131.

53. Sacchetti, C.; Motamedchaboki, K.; Magrini, A.; Palmieri, G.; Mattei, M.; Bernardini, S.; Rosato, N.; Bottini, N.; Bottini, M. Surface polyethylene glycol conformation influences the protein corona of polyethylene glycol-modified single-walled carbon nanotubes: Potential implications on biological performance. ACS Nano 2013, 7, 1974-1989.

54. Erlanger, B.F.; Chen, B.-X.; Zhu, M.; Brus, L. Binding of an anti-fullerene IgG monoclonal antibody to single wall carbon nanotubes. Nano Lett. 2001, 1, 465-467.

55. Dieckmann, G.R.; Dalton, A.B.; Johnson, P.A.; Razal, J.; Chen, J.; Giordano, G.M.; Muñoz, E.; Musselman, I.H.; Baughman, R.H.; Draper, R.K. Controlled assembly of carbon nanotubes by designed amphiphilic Peptide helices. J. Am. Chem. Soc. 2003, 125, 1770-1777.

56. Zorbas, V.; Ortiz-Acevedo, A.; Dalton, A.B.; Yoshida, M.M.; Dieckmann, G.R.; Draper, R.K.; Baughman, R.H.; Jose-Yacaman, M.; Musselman, I.H. Preparation and characterization of individual peptide-wrapped single-walled carbon nanotubes. J. Am. Chem. Soc. 2004, 126, 7222-7227.

57. Karajanagi, S.S.; Yang, H.; Asuri, P.; Sellitto, E.; Dordick, J.S.; Kane, R.S. Protein-assisted solubilization of single-walled carbon nanotubes. Langmuir 2006, 22, 1392-1395. 
58. Li, X.; Chen, W.; Zhan, Q.; Dai, L.; Sowards, L.; Pender, M.; Naik, R.R. Direct measurements of interactions between polypeptides and carbon nanotubes. J. Phys. Chem. B 2006, 110, 12621-12625.

59. Salvador-Morales, C.; Townsend, P.; Flahaut, E.; Vénien-Bryan, C.; Vlandas, A.; Green, M.L.H.; Sim, R.B. Binding of pulmonary surfactant proteins to carbon nanotubes; potential for damage to lung immune defense mechanisms. Carbon 2007, 45, 607-617.

60. Witus, L.S.; Rocha, J.-D.R.; Yuwono, V.M.; Paramonov, S.E.; Weismana, R.B.; Hartgerink, J.D. Peptides that non-covalently functionalize single-walled carbon nanotubes to give controlled solubility characteristics. J. Mater. Chem. 2007, 17, 1909-1915.

61. Nepal, D.; Geckeler, K.E. Proteins and carbon nanotubes: Close encounter in water. Small 2007, 3, 1259-1265.

62. Wang, S.; Humphreys, E.S.; Chung, S.Y.; Delduco, D.F.; Lustig, S.R.; Wang, H.; Parker, K.N.; Rizzo, N.W.; Subramoney, S.; Chiang, Y.M. Peptides with selective affinity for carbon nanotubes. Nat. Mater. 2003, 2, 196-200.

63. Zuo, G.; Huang, Q.; Wei, G.; Zhou, R.; Fang, H. Plugging into proteins: Poisoning protein function by a hydrophobic nanoparticle. ACS Nano 2010, 4, 7508-7514.

64. Yi, C.; Fong, C.C.; Zhang, Q.; Lee, S.T.; Yang, M. The structure and function of ribonuclease A upon interacting with carbon nanotubes. Nanotechnology 2008, 19, 095102.

65. Zhang, B.; Xing, Y.; Li, Z.; Zhou, H.; Mu, Q.; Yan, B. Functionalized carbon nanotubes specifically bind to alpha-chymotrypsin's catalytic site and regulate its enzymatic function. Nano Lett. 2009, 9, 2280-2284.

66. Karajanagi, S.S.; Vertegel, A.A.; Kane, R.S.; Dordick, J.S. Structure and function of enzymes adsorbed onto single-walled carbon nanotubes. Langmuir 2004, 20, 11594-11599.

67. Shen, J.W.; Wu, T.; Wang, Q.; Kang, Y. Induced stepwise conformational change of human serum albumin on carbon nanotube surfaces. Biomaterials 2008, 29, 3847-3855.

68. Azamian, B.R.; Davis, J.J.; Coleman, K.S.; Bagshaw, C.B.; Green, M.L. Bioelectrochemical single-walled carbon nanotubes. J. Am. Chem. Soc. 2002, 124, 12664-12665.

69. Bussy, C.; Kostarelos, K. Carbon nanotubes in medicine and biology-Safety and toxicology. Adv. Drug Deliv. Rev. 2013, 65, 2061-2062.

70. Gasser, M.; Rothen-Rutishauser, B.; Krug, H.F.; Gehr, P.; Nelle, M.; Yan, B.; Wick, P. The adsorption of biomolecules to multi-walled carbon nanotubes is influenced by both pulmonary surfactant lipids and surface chemistry. J. Nanobiotechnology 2010, 8, 31.

71. Gasser, M.; Wick, P.; Clift, M.J.; Blank, F.; Diener, L.; Yan, B.; Gehr, P.; Krug, H.F.; Rothen-Rutishauser, B. Pulmonary surfactant coating of multi-walled carbon nanotubes (MWCNTs) influences their oxidative and pro-inflammatory potential in vitro. Part. Fibre Toxicol. 2012, 9, 17.

72. Holt, B.D.; Dahl, K.N.; Islam, M.F. Cells take up and recover from protein-stabilized single-wall carbon nanotubes with two distinct rates. ACS Nano 2012, 6, 3481-3490. 
73. Holt, B.D.; Dahl, K.N.; Islam, M.F. Quantification of uptake and localization of bovine serum albumin-stabilized single-wall carbon nanotubes in different human cell types. Small 2011, 7, 2348-2355.

74. Kostarelos, K.; Lacerda, L.; Pastorin, G.; Wu, W.; Wieckowski, S.; Luangsivilay, J.; Godefroy, S.; Pantarotto, D.; Briand, J.P.; Muller, S. Cellular uptake of functionalized carbon nanotubes is independent of functional group and cell type. Nat. Nanotechnol. 2007, 2, 108-113.

75. Angelikopoulos, P.; Schou, K.; Bock, H. Surfactant-induced forces between carbon nanotubes. Langmuir 2010, 26, 18874-18883.

76. Salvador-Morales, C.; Basiuk, E.V.; Basiuk, V.A.; Green, M.L.; Sim, R.B. Effects of covalent functionalization on the biocompatibility characteristics of multi-walled carbon nanotubes. J. Nanosci. Nanotechnol. 2008, 8, 2347-2356.

77. Lin, Y.; Allard, L.F.; Sun, Y.P. Protein-affinity of single-walled carbon nanotubes in water. J. Phys. Chem. B 2004, 108, 3760-3764.

78. Shim, M.; Kam, N.W. S.; Chen, R.J.; Li, Y.; Dai, H. Functionalization of carbon nanotubes for biocompatibility and biomolecular recognition. Nano Lett. 2002, 2, 285-288.

79. Cai, X.; Ramalingam, R.; Wong, H.S.; Cheng, J.; Ajuh, P.; Cheng, S.H.; Lam, Y.W. Characterization of carbon nanotube protein corona by using quantitative proteomics. Nanomedicine 2013, 9, 583-593.

80. Shannahan, J.H.; Brown, J.M.; Chen, R.; Ke, P.C.; Lai, X.; Mitra, S.; Witzmann, F.A. Comparison of nanotube-protein corona composition in cell culture media. Small 2013, 9, 2171-2181.

81. Mu, Q.; Liu, W.; Xing, Y.; Zhou, H.; Li, Z.; Zhang, Y.; Ji, L.; Wang, F.; Si, Z.; Zhang, B.; Yan, B. Protein binding by functionalized multiwalled carbon nanotubes is governed by the surface chemistry of both parties and the nanotube diameter. J. Phys. Chem. C 2008, 112, 3300-3307.

82. Chen, R.J.; Zhang, Y.; Wang, D.; Dai, H. Noncovalent sidewall functionalization of single-walled carbon nanotubes for protein immobilization. J. Am. Chem. Soc. 2001, 123, 3838-3839.

83. Bradley, K.; Briman, M.; Star, A.; Grüner, G. Charge transfer from adsorbed proteins. Nano Lett. 2004, 4, 253-256.

84. Kam, N.W.; Liu, Z.; Dai, H. Carbon nanotubes as intracellular transporters for proteins and DNA: An investigation of the uptake mechanism and pathway. Angew. Chem. Int. Ed. Engl. 2006, 45, 577-581.

85. Ling, W.L.; Biro, A.; Bally, I.; Tacnet, P.; Deniaud, A.; Doris, E.; Frachet, P.; Schoehn, G.; Pebay-Peyroula, E.; Arlaud, G.J. Proteins of the innate immune system crystallize on carbon nanotubes but are not activated. ACS Nano 2011, 5, 730-737.

86. Holt, B.D.; McCorry, M.C.; Boyer, P.D.; Dahl, K.N.; Islam, M.F. Not all protein-mediated single-wall carbon nanotube dispersions are equally bioactive. Nanoscale 2012, 4, 7425-7434. 
87. Tenzer, S.; Docter, D.; Rosfa, S.; Wlodarski, A.; Kuharev, J.; Rekik, A.; Knauer, S.K.; Bantz, C.; Nawroth, T.; Bier, C.; et al. Nanoparticle size is a critical physicochemical determinant of the human blood plasma corona: A comprehensive quantitative proteomic analysis. ACS Nano 2011, 5, 7155-7167.

88. Tenzer, S.; Docter, D.; Kuharev, J.; Musyanovych, A.; Fetz, V.; Hecht, R.; Schlenk, F.; Fischer, D.; Kiouptsi, K.; Reinhardt, C.; et al. Rapid formation of plasma protein corona critically affects nanoparticle pathophysiology. Nat. Nanotechnol. 2013, 8, 772-781.

89. Dobrovolskaia, M.A.; Patri, A.K.; Zheng, J.; Clogston, J.D.; Ayub, N.; Aggarwal, P.; Neun, B.W.; Hall, J.B.; McNeil, S.E. Interaction of colloidal gold nanoparticles with human blood: Effects on particle size and analysis of plasma protein binding profiles. Nanomedicine 2009, 5, 106-117.

90. Schäffler, M.; Semmler-Behnke, M.; Sarioglu, H.; Takenaka, S.; Wenk, A.; Schleh, C.; Hauck, S.M.; Johnston, B.D.; Kreyling, W.G. Serum protein identification and quantification of the corona of 5, 15 and $80 \mathrm{~nm}$ gold nanoparticles. Nanotechnology 2013, 24, 265103.

91. Maiorano, G.; Sabella, S.; Sorce, B.; Brunetti, V.; Malvindi, M.A.; Cingolani, R.; Pompa, P.P. Effects of cell culture media on the dynamic formation of protein-nanoparticle complexes and influence on the cellular response. ACS Nano 2010, 4, 7481-7491.

92. Lundqvist, M.; Stigler, J.; Elia, G.; Lynch, I.; Cedervall, T.; Dawson, K.A. Nanoparticle size and surface properties determine the protein corona with possible implications for biological impacts. Proc. Natl. Acad. Sci. USA 2008, 105, 14265-14270.

93. Lind, K.; Kresse, M.; Müller, R.H. Comparison of protein adsorption patterns onto differently charged hydrophilic superparamagnetic iron oxide particles obtained in vitro and ex vivo. Electrophoresis 2001, 22, 3514-3521.

94. Jansch, M.; Stumpf, P.; Graf, C.; Rühl, E.; Müller, R.H. Adsorption kinetics of plasma proteins on ultrasmall superparamagnetic iron oxide (USPIO) nanoparticles. Int. J. Pharm. 2012, 428, 125-133.

95. Gagner, J.E.; Lopez, M.D.; Dordick, J.S.; Siegel, R.W. Effect of gold nanoparticle morphology on adsorbed protein structure and function. Biomaterials 2011, 32, 7241-7252.

96. Gagner, J.E.; Qian, X.; Lopez, M.M.; Dordick, J.S.; Siegel, R.W. Effect of gold nanoparticle structure on the conformation and function of adsorbed proteins. Biomaterials 2012, 33, 8503-8516.

97. Vertegel, A.A.; Siegel, R.W.; Dordick, J.S. Silica nanoparticle size influences the structure and enzymatic activity of adsorbed lysozyme. Langmuir 2004, 20, 6800-6807.

98. Deng, J.; Sun, M.; Zhu, J.; Gao, C. Molecular interactions of different size AuNP-COOH nanoparticles with human fibrinogen. Nanoscale 2013, 5, 8130-8137.

99. Asuri, P.; Karajanagi, S.S.; Yang, H.; Yim, T.J.; Kane, R.S.; Dordick, J.S. Increasing protein stability through control of the nanoscale environment. Langmuir 2006, 22, 5833-5836.

100. Deng, Z.J.; Mortimer, G.; Schiller, T.; Musumeci, A.; Martin, D.; Minchin, R.F. Differential plasma protein binding to metal oxide nanoparticles. Nanotechnology 2009, 20, 455101. 


\title{
Elucidating the Potential Biological Impact of Cellulose Nanocrystals
}

\section{Sandra Camarero-Espinosa, Carola Endes, Silvana Mueller, Alke Petri-Fink, Barbara Rothen-Rutishauser, Christoph Weder, Martin James David Clift and E. Johan Foster}

\begin{abstract}
Cellulose nanocrystals exhibit an interesting combination of mechanical properties and physical characteristics, which make them potentially useful for a wide range of consumer applications. However, as the usage of these bio-based nanofibers increases, a greater understanding of human exposure addressing their potential health issues should be gained. The aim of this perspective is to highlight how knowledge obtained from studying the biological impact of other nanomaterials can provide a basis for future research strategies to deduce the possible human health risks posed by cellulose nanocrystals.
\end{abstract}

Reprinted from Fibers. Cite as: Camarero-Espinosa, S.; Endes, C.; Mueller, S.; Petri-Fink, A.; Rothen-Rutishauser, B.; Weder, C.; Clift, M.J.D.; Foster, E.J. Elucidating the Potential Biological Impact of Cellulose Nanocrystals. Fibers 2016, 4, 21.

\section{Introduction}

Cellulose is the most abundant organic polymer on earth, and can be found in plants, algae, bacteria, amoeba, and even some marine animals. The polymer is composed of $\beta-(1 \rightarrow 4)$ D-glucose monomers [1], and in its natural state, cellulose is a hierarchically structured material with different layers of organization. At the lowest level, the polymer chains are organized in highly ordered and uniaxially oriented crystalline domains, which are disrupted by disordered amorphous regions. This structure is the basis for the isolation of different types of nanocellulose from natural cellulosic materials.

Several distinct forms of nanocellulose types, where at least one of the dimensions is on the nano-scale, exist. The most commonly studied and used forms are bacterial cellulose (BC), microcrystalline cellulose (MC), microfibrillated cellulose (MFC) and cellulose nanocrystals (CNCs) [2,3]. CNCs, which are also referred to as cellulose nanowhiskers (CNWs) or nanocrystalline cellulose (NCC), are produced by hydrolysis of cellulose pulp with a mineral acid, such as hydrochloric acid [4], sulphuric acid [5] or phosphoric acid [6]. During the acid treatment, the amorphous portions of the hierarchically structured material, which are more prone to hydrolysis than the crystalline domains, are disintegrated so that only the crystalline parts remain in the form of 'needle-shaped' nanofibers. Cellulose nanocrystals thus made 
exhibit a length between hundred $\mathrm{nm}$ and several $\mu \mathrm{m}$ and a width between 10 and $50 \mathrm{~nm}[7,8]$, dependent on the cellulose source used [9].

CNCs are receiving considerable interest within the research community due to their interesting and desirable set of properties, which include the renewable nature of their sources, and a combination of high stiffness and strength and low density [2]. Thus, CNCs have been widely used as reinforcing filler for a variety of polymers to yield nanocomposites with improved mechanical properties $[2,3,10]$. In addition, the surface chemistry, made up almost exclusively of hydroxyl groups, renders nanocellulose as an interesting substrate whose surface can be readily and freely functionalized. This propensity, together with their biologically benign nature, is driving the use of nanocellulose within different (bio)materials [10-12]. $\mathrm{CNC}$ have further been used in a broad range of other new materials applications, including optically [13] and electrically [14,15] active materials, aerogels [16-18], and mechanically adaptive materials [7,19-25], just to name a few examples.

Fueled by promising outcomes of research projects, and great potential of pilot studies, an industrial-scale production of CNCs is being undertaken [26], and commercial exploitation of this nanomaterial has begun. Whilst such an outlook can be seen as advantageous from an application point of view, i.e., new materials that are cost-effective and that provide advanced, as well as enhanced qualities over their alternative counterparts, there remain open questions [27] concerning the human exposure to CNC-based nanomaterials, and furthermore, what the (potentially adverse) human health effects are following such an exposure.

Over the past three decades, during which the field of nanotechnology witnessed constant expansion, there has been heightened emphasis placed upon the need to develop a thorough understanding of the biological impact of nano-sized materials. Although the above highlighted examples illustrate the potential effectiveness of nanocellulose as an application, there remains a necessity to holistically deduce their possible adverse biological impact due to their nanoscale properties [28], taking into consideration the pitfalls associated with studying possible nanomaterial hazard [29]. Thus, with nanocellulose, it is essential to build upon the already formed knowledgebase of nanomaterial hazard, even via read-across techniques, wherein structurally similar analogues are used to hypothesize toxicity without experimental testing [30], in order to progress both understanding and perception of the biological impact of such 'new' nanomaterials effectively.

The objective of this perspective is, therefore, to consider how the advancements of nanocellulose applications have been studied through both in vitro and in vivo investigation, and how this knowledge within may be attributed towards clarity of current understanding, and future activities regarding the use of, and biological impact of CNCs. 


\section{Life-Cycle and Human Exposure of CNCs}

As with any other (biodegradable) material, CNCs have a life-cycle [31,32] which, as shown in Figure 1, is initiated with the growth and harvesting of the natural raw material (the most viable source for commercial use at this point appears to be wood, although for research purposes many other sources are being used, including cotton $[6,33,34]$, banana stems [8], and tunicates) $[7,35]$ and continues with its isolation, the modification and integration into a material system (e.g., compounding with a polymer), and further processing in order to create a final 'product', which, eventually, is placed on the market. The life-cycle continues thereafter with further processing prior to disposal, which may occur through biodegradation or incineration. Throughout this life-cycle, there is the possibility of exposure to humans, eventually after nanocellulose is released from the product and through a number of environments and scenarios. In each of these there are different modes of human exposure, which include the respiratory tract (inhalation), skin contact, eye contact, ingestion and possible interaction with the bloodstream (i.e., via direct injection through medical application, or via translocation from the lung following inhalation $[32,36]$ ) resulting in possible secondary organ exposure, i.e., liver, heart, brain, and/or kidney.

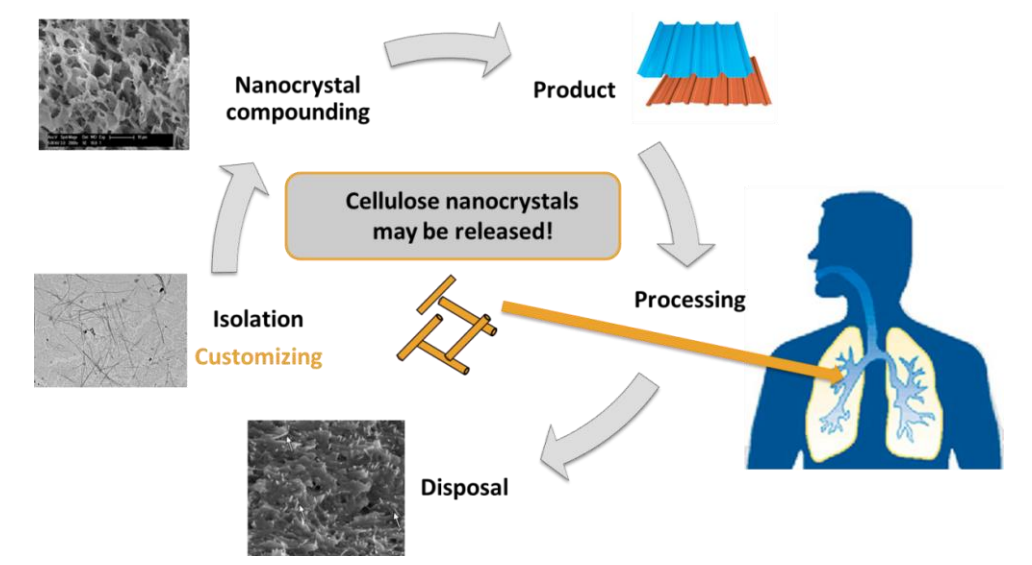

Figure 1. Schematic of the life-cycle of cellulose nanocrystals and products made from these nanoparticles. There are five main points in the life-cycle of CNCs; i. isolation, ii. compounding, iii. product formation, vi. post manufacturing processing and use, and v. disposal. All stages of the life-cycle pose a potential human exposure scenario for which both the exposure level and the hazard associated, and thus the risk of CNCs to human health, are currently not fully understood. It must be emphasized that inhalation exposure remains the assumed primary route of entry to the human body for CNCs. 
However, only two major exposure routes have been observed as pertinent to humans during life-cycles involving anisotropically shaped nanomaterials of this type; inhalation and skin exposure. This knowledge originates from studies by Maynard and colleagues [37], as well as more recently by others [38], involving carbon nanotubes (CNTs) and not CNCs. Due to the significant differences between the production, properties and anticipated fields of use of CNTs [39] and nanocellulose [3], it must be considered that the exposure routes towards humans could be different, although one can speculate that inhalation probably would remain the primary form of uptake due to the potential aerosolisation of the CNCs at this point in their life-cycle. A pertinent association could also be made with the isolation of bulk cotton fibres [40], although this would arguably only be relevant to cotton-based $\mathrm{CNCs}$, the exposure risk and routes remain the same (i.e., inhalation and skin exposure). Naturally, if workers are adequately protected then such exposures can be reduced [41]. However, despite such attention to worker safety, since workers would be exposed to repeated doses of nanocellulose, over a chronic period of time such an understanding is necessary, as is the specific concentrations that they are exposed to. Therefore, to progress knowledge in this area, (i) the human exposure routes must be confirmed for CNCs at the isolation stage of their life-cycle; and furthermore (ii) understanding of the occupational exposure levels should be confirmed.

In order to determine the human exposure routes within a nanocellulose production environment, a number of lessons can be learned from air pollution, as well as those studies focusing on other nanomaterials [42,43]. It must be noted, however, that the specific identification of aerosolised or otherwise released nanomaterial fractions, especially fibrous nanomaterials, are highly problematic and such particles are difficult to measure in any environment due to limitations in the currently available technology, e.g., with a scanning mobility particle sizer (SMPS) [44]. It is currently unknown to what extent CNCs can be detected with available methods. Thus, as a starting point, it would be important to confirm the usefulness of existing analytical tools or develop new methodologies that permit the accurate measurement of the actual CNC concentration in air, so that these particles can be detected efficiently right from their origin.

The issue of human exposure levels to nanomaterials is, in general, an important issue within the field of nanotoxicology. Recently, intense efforts have been made by the National Institute for Occupational Safety and Health (NIOSH) in the United States of America. Although it has established occupational exposure levels for silica dust and titanium dioxide, NIOSH has predominantly focused on CNTs, and in a recent central intelligence bulletin suggested an exposure limit for CNTs as $1 \mu \mathrm{g} / \mathrm{m}^{3}$ for an eight hour working day [45]. Although this recommended exposure limit (REL) could be considered as an overload situation over a workers' life-time [46], 
this metric has been suggested based on a plethora of in vivo and some in vitro testing strategies using solely CNTs in order to comprehend specificity for these nanomaterials. This concept therefore reduces somewhat the applicability towards an REL for CNCs. However, if the physical characteristics of the nanocellulose sample in question are remotely comparable to those of the CNTs, then it could be, or might be considered apt. Nonetheless, the US Occupational Safety and Health Administration had previously set a specific permissible exposure limit (PEL) of $200-750 \mathrm{\mu g} / \mathrm{m}^{3}$ over an eight hour timed weighted average (TWA) for cotton dust. Irrespective of the issues surrounding both exposure limits, they do provide a significant basis for research to dictate that investigations undertake exposures at 'realistic' concentrations/doses so that extrapolation towards human exposure can be made [47]. Furthermore, such exposure limit values provide a valuable 'stop-gap' until regulatory bodies are able to provide direction towards the use and exposure of nanomaterials [48]. It is also prudent to note that the REL TWA provided by NIOSH for silica dust $\left(0.05 \mathrm{mg} / \mathrm{m}^{3}\right)$ [49] could also be used as a 'highest exposure scenario' for CNCs, due to the heightened crystalline fraction (which is the fraction known to drive the heightened inflammatory responses caused following (most) silica exposures) [50]. This concept further highlights an important note, in general, for the nanotox community regarding the need for the appropriate use of positive particle controls to use as a comparison for determining the biological impact of nanomaterials, such as CNCs.

For the subsequent compounding and usage (i.e., product) of CNC-based materials there is also a risk of exposure, albeit it can be assumed to be much smaller than during the initial isolation of CNCs. During these latter stages of the life-cycle, the risk of exposure can mostly be attributed towards the possible abrasion of the product, which could result in the release of individual CNCs, small CNC aggregates, or nanocellulose-polymer composite (nano)particles, which could be subsequently inhaled or penetrate through the skin upon contact. Recent research on this matter has again focused upon CNTs [51-53]. From these initial studies it has been postulated that the release of CNTs, at least in their bare form and also combined with polymer matrix is relatively low. Specific exposure levels are not yet known and therefore additional research must be conducted. Furthermore, in terms of usage, it should also be noted that there could be direct exposure to the human body via ingestion (e.g., nanocellulose in contact with food products, such as in food packaging) and also there is the potential injection into the human bloodstream (e.g., the use of nanocellulose as a tool within nanomedicine). These latter aspects, however, are currently of minor importance, as the use of nanocellulose as main components in such food-related and/or medical devices do not appear to be imminent. However, due to their potential application in these contexts, hazard assessment of these scenarios should be undertaken in order to obtain clear 
risk analysis data, as previously shown by Bergin and Witzmann (ingestion of nanomaterials) [54], as well as for medical application (i.e., injection) [55].

Finally, understanding of the human exposure effects during the disposal of nanocellulose, in whatever format, is severely limited. A recent study into the incineration of nanomaterials in a waste plant showed that at a variety of different locations within the building, no or only small amounts of nanomaterials were found following their incineration [56]. Whilst this could also be true for nanocellulose, it is safe to assume that, very much like wood, cotton and other raw cellulosic materials from which CNCs are extracted will end in similar ash once burnt.

Thus, from the currently available information and relevant application of nanocellulose, it can be summarized that during the entire life-cycle the human exposure routes can be stated in order of importance as i.e., inhalation $>$ skin $>$ others (e.g., eye contact, ingestion, injection). Such a perspective is vital towards determining which exposure route hazard analyses should focus upon. This however, is by no means new information. It can be considered that the entire discipline of nanotoxicology is predominantly based upon the consideration that most nanomaterials are inhaled and therefore the lung is the primary human target organ, as is the case within the particle toxicology field [57]. However, when focusing upon these exposure routes, emphasis should be upon which forms of nanocellulose to study. Since the potential for inhalation of nanocellulose is most paramount at the isolation stage, it is fundamental that the biological impact of bare and functionalised CNCs are studied initially. Such information would then act as a building block in assessing the hazard posed by nanocellulose released from polymer composites (or a combination thereof), and subsequently the human health implications during their disposal. For the success of such an outlook however, all nanocelluose samples would need to undergo essential and thorough characterization.

\section{Characterising CNC Exposure}

Since the mid-2000s, it has been necessary that a thorough characterisation of the specific, pristine nanomaterial being testing for their biological impact is performed [58]. In fact, it is mandatory for most journals nowadays that such information is contained within all original research manuscripts. This significant change within the field of nanotoxicology is evident from the continual association and significant influence that the physico-chemical characteristics of nanomaterials were noted to (significantly) contribute to the biological effects observed [59]. Although widely accepted, this concept did however raise multiple discussions as to which physical and/or chemical characteristics must be studied for each nanomaterial. Due to the diverse nature of nanomaterials, it has so far been too difficult to define a precise set of characterisation standards (i.e., which characteristics must researchers assess?). Mostly the characteristics of shape, size, (chemical) 
composition, surface material, surface charge density and surface area [58] have been considered paramount. However, due to analytical challenges associated with some nanomaterials [60] it has predominantly been accepted that as much information on the physico-chemical characteristics are provided as possible. Furthermore, assessment of the physico-chemical characteristics within the biological environment (e.g., for in vitro based investigations, it is important to determine the impact that the cell culture medium and associated proteins has upon nanocellulose) studied is desirable [61], yet challenging [62].

Currently there is limited understanding as to the biological impact of nanocellulose in relation to their physical attributes (throughout their life-cycle), thus developing such knowledge will lend itself to determining their biocompatibility. Furthermore, such information is important for the future of nanocellulose hazard assessment, since in a number of previous studies an intimate characterisation is unfortunately absent, making it difficult to correlate across different studies and to address, if any, the key parameters that influence different cell responses following nanocellulose exposure [10].

In order to address this, Table 1 highlights many of the key physico-chemical parameters that should, ideally, be investigated when studying nanocellulose and CNCs in particular. Furthermore, the problems associated with each different technique and analytical endpoint is highlighted, with subsequent suggestions as to how to mitigate such issues. Although all the parameters highlighted in Table 1 are essential, it is again important to note that the potential hazard of CNCs would likely be related to (i) their dimensions (i.e., in the nanoscale); and (ii) their 'fibre-like' appearance (i.e., long, straight, and often 'needle-like'). Whilst the first hurdle, their nanoscale dimension, is suitably covered by the suggested analyses given in Table 1, the latter (i.e., fibre-like appearance) can be related to the 'fibre paradigm' [63].

The fibre paradigm itself is associated with the findings of both glass [64] and asbestos fibers [63]. It was originally shown by Davis and colleagues [65] that long, stiff amosite asbestos fibers, unlike short amosite asbestos fibres, can lead to serious damage to the lungs of rats when inhaled or following intraperitoneal injection. Effects noted were chronic inflammation leading to eventual granuloma formation and in some cases mesothelioma (the hallmark cancer of long fibre asbestos exposure). In regards to glass fibers, often used in construction as an insulating material and fire retardant, similar heightened negative health effects towards both workers and consumers have been shown over an increased period [66]. Further research has shown that the specific health related issues following exposure to both glass and asbestos fibres include inflammation, alveolitis and reduced pulmonary functions [67]. Importantly, all of this work could only be reported in the manner it was due to the specific physical and chemical characterisation of the fibres investigated. More recently, CNTs, which are potentially advantageous components 
for a number of different consumer, industrial, and technological applications, were shown to induce asbestos-like effects when introduced into the peritoneal cavity of mice [68]. These results however were attributed to specific physicochemical characteristics i.e., increased length and stiffness as well as biopersistence.

For CNCs, concerns associated with the fibre paradigm are debatable as their average lengths do not fit the required characteristics to fit the paradigm [63]. Indeed, the minimum length for nanomaterials, or high aspect ratio nanomaterials (HARN), to fit the fibre paradigm is $>5 \mu \mathrm{m}$ [69]. Average dimensions for typical CNCs isolated from cotton $(100-200 \times 5-15 \mathrm{~nm})$ soft-wood pulp $(100-150 \times 5-15 \mathrm{~nm})$, and tunicates (1000-2000 × 10-20 nm) are significantly below this threshold [70]. This is, however, not to say that the population of fibres that are longer that $5 \mu \mathrm{m}$ is zero (especially in long CNC types such as tunicate CNCs [71]) and that therefore such materials should not elucidate effects associated with the fibre-paradigm. Indeed, this aspect suggests that CNCs demand special attention considering their proposed application and possible human exposure. Further need to study nanocellulose in this notion is that their width $(<5 \mu \mathrm{m})$ certainly fits the fibre paradigm [63]. The final aspect of this paradigm however, which remains the most difficult to decipher for any (nano)fibre type, especially nanocellulose, is their biopersistance (or biodurability [72]).

Table 1. Overview of the most commonly used analytical methods for the characterization of the physico-chemical properties of nanocellulose, in particular CNCs. Details as to the limitations of each method, with concepts towards mitigation of such limitations also given.

\begin{tabular}{|c|c|c|c|c|}
\hline $\begin{array}{l}\text { Characterization } \\
\text { Method }\end{array}$ & $\begin{array}{l}\text { Feature of } \\
\text { Nanocellulose } \\
\text { Characterised }\end{array}$ & $\begin{array}{l}\text { Limitation Regarding } \\
\text { Nanocellulose }\end{array}$ & Limitation Mitigation & References \\
\hline $\begin{array}{l}\text { Electron } \\
\text { Microscopy (TEM) }\end{array}$ & $\begin{array}{l}\text { Shape \& dimension } \\
\text { (Best for overall } \\
\text { structural analysis, } \\
\text { for most samples) }\end{array}$ & $\begin{array}{l}\text { Drying effects when } \\
\text { spotting onto EM grids }\end{array}$ & $\begin{array}{l}\text { Alter drying conditions, } \\
\text { concentration, BSA-based } \\
\text { techniques [73] }\end{array}$ & {$[6,13,74]$} \\
\hline $\begin{array}{l}\text { Atomic Force } \\
\text { Microscopy (AFM) }\end{array}$ & Shape \& dimension & $\begin{array}{l}\text { AFM tip has the } \\
\text { potential to } \\
\text { overestimate sizes if } \\
\text { sharpness is lost }\end{array}$ & $\begin{array}{l}\text { Use height (more } \\
\text { accurate), not } \\
\text { measured width }\end{array}$ & [75-77] \\
\hline $\begin{array}{l}\text { Dynamic } \\
\text { Light-Scattering (DLS) }\end{array}$ & Overall dimensions & $\begin{array}{l}\text { Tough to elucidate } \\
\text { exact dimensions }\end{array}$ & $\begin{array}{l}\text { Modify with } \\
\text { an accurate form factor }\end{array}$ & {$[78,79]$} \\
\hline Optical Photographs & $\begin{array}{l}\text { Dispersion/colloidal } \\
\text { stability. Observation } \\
\text { of aggregates (larger } \\
\text { than } 300 \mathrm{~nm} \text { ) }\end{array}$ & $\begin{array}{l}\text { Limited by Abbe } \\
\text { diffraction limit }\end{array}$ & $\begin{array}{l}\text { Must use electron } \\
\text { microscopy for smaller } \\
\text { (less than } 300 \mathrm{~nm} \text { ) }\end{array}$ & {$[80,81]$} \\
\hline $\begin{array}{l}\text { Conductometric } \\
\text { Charge Titration }\end{array}$ & $\begin{array}{l}\text { Charge density (Best for } \\
\text { surface half ester content } \\
\text { determination) }\end{array}$ & $\begin{array}{l}\text { Small }(<20 \mathrm{mmol} / \mathrm{Kg}) \\
\text { is within noise limit }\end{array}$ & Larger sample size, & {$[6,82]$} \\
\hline
\end{tabular}


Table 1. Cont.

\begin{tabular}{|c|c|c|c|c|}
\hline $\begin{array}{l}\text { Characterization } \\
\text { Method }\end{array}$ & $\begin{array}{l}\text { Feature of } \\
\text { Nanocellulose } \\
\text { Characterised }\end{array}$ & $\begin{array}{l}\text { Limitation Regarding } \\
\text { Nanocellulose }\end{array}$ & Limitation Mitigation & References \\
\hline Elemental Analysis & $\begin{array}{l}\text { Elemental content } \\
\text { of sample }\end{array}$ & $\begin{array}{l}\text { Common for } \mathrm{C}, \mathrm{H}, \mathrm{N}, \\
\text { S, P analysis only }\end{array}$ & $\begin{array}{l}\text { Must be correlated to } \\
\text { predicted } \\
\text { chemical structure }\end{array}$ & {$[6,83,84]$} \\
\hline $\begin{array}{l}\text { Infrared } \\
\text { Spectroscopy (IR) }\end{array}$ & $\begin{array}{l}\text { Functional } \\
\text { groups (bonds) }\end{array}$ & $\begin{array}{l}\text { Only looks at } \\
\text { chemical bonds }\end{array}$ & $\begin{array}{l}\text { Limited to IR active } \\
\text { chemical } \\
\text { bonds, sensitivity }\end{array}$ & {$[78,85]$} \\
\hline $\begin{array}{l}\text { X-ray Photoelectron } \\
\text { Spectroscopy }\end{array}$ & Elements on the surface & $\begin{array}{l}\text { Voxel does not allow } \\
\text { individual } \\
\text { CNC analysis }\end{array}$ & $\begin{array}{l}\text { Does not elucidate } \\
\text { groups, only elements }\end{array}$ & [86] \\
\hline $\begin{array}{l}\text { Brunauer, Emmet and } \\
\text { Teller method (BET) }\end{array}$ & Surface area & $\begin{array}{l}\text { Cellulose naturally } \\
\text { aggregates when dried }\end{array}$ & $\begin{array}{l}\text { Aggregation will } \\
\text { lead to lower than } \\
\text { individualized CNCs }\end{array}$ & {$[87,88]$} \\
\hline Dye Adhesion & Surface area & Limited by size of dye & $\begin{array}{l}\text { Use in conjunction with } \\
\text { other techniques } \\
\text { (e.g., rough estimation } \\
\text { by length } \times \\
\text { dimension analysis) }\end{array}$ & {$[70,89]$} \\
\hline $\begin{array}{l}\text { Inverse Gas } \\
\text { Chromatography } \\
\text { (IGC) }\end{array}$ & Surface properties & $\begin{array}{l}\text { Cellulose naturally } \\
\text { aggregates when dried }\end{array}$ & $\begin{array}{l}\text { Aggregation will } \\
\text { lead to lower than } \\
\text { individualized CNCs }\end{array}$ & [71] \\
\hline
\end{tabular}

Previously, biopersistence (associated with exposure to the human lung) of any fibrous material has been deduced via direct assessment (mostly in acellular and in vivo environments) over a chronic period. However, in order to reduce animal experimentation in vitro based analyses have also previously focused on a single-cell system (e.g., macrophages), as well as using different biological-based buffers at different $\mathrm{pH}$ and under flow conditions. Yet, despite these efforts there is currently no clear method to efficiently and effectively elucidate the potential biopersistence or biodurability of a (nano)fibre. Currently, knowledge of the biopersistance, or biodurability of nanocellulose is severely lacking, although several studies are ongoing [31], in order to truly understand its biological impact, efforts must be made to comprehend this important biological-based characteristic of the material.

\section{How to Determine the Potential Biological Impact of Nanocellulose}

The biological impact of the bulk form of non-nanoscale cellulose fibres [90] (e.g., microfibrilated nanocellulose) as well as cellulose dust (usually micron sized $(>10 \mu \mathrm{m}))$ has been widely studied in the past [91]. Due to the inherent differences between these materials and nanocellulose, it is difficult to make any clear correlations between them. However, it must be emphasized that non-nanosized cellulose materials, when compared to other fibrous types, such as asbestos, commonly showed limited adverse biological effects [92]. 
Focusing upon CNCs however, a first study on the biological impact of CNCs isolated from cotton and tunicates was reported by Clift and colleagues in 2011 [93]. Further insightful research studies have followed (Table 2), and have contributed to the current understanding of the biological impact of CNCs and other nanocellulose types. Despite the increasing number of studies published on this new nanomaterial in the past few years, in most of the cases a first biocompatibility analysis was performed to assess the possible lethality of the nanocellulose. Yet, a detailed mechanistic toxicological assessment that is necessary to determine their potential human health effects (over time) remains lacking. Such analyses are vital, especially considering the landmarks of nanotoxicological research strategies; i.e., considering the potential for nanocellulose to cause oxidative stress [31,94,95], and possibly genotoxicity [96]. Such understanding is imperative towards conceiving any understanding as to the potential (chronic) adverse effects of nanocellulose towards human health. Thus, in order to achieve such investigations representative models must be utilized, and often in collaboration with other, complimentary testing strategies.

Commonly, in the past, in vivo studies (e.g., rodent models) have been used to study the toxicology of nanomaterials, since these allow for whole body exposure scenarios and permit assessing the biodurability, dissolution and secondary organ toxicity of any test substance. However, in view of the recent calls for the refinement and reduction of such animal based testing strategies (with a view to eventually replacing them over time) there is an immediate need to develop alternative testing models, such as in vitro, in silico and computational models [97]. In a recent review, Hartung and Sabbioni highlighted the 'alternative' models currently available within the field of nanotoxicology [98]. Recently, several advanced and multi-cellular in vitro systems have been used with the objective to determine the mechanisms behind the possible hazard associated with nanomaterials [99]. Whilst these models show a different biochemical/biomolecular response to monoculture systems (whether it be a similar trend, but different concentration-based effects, or a completely different biological effect) [100], there is still much debate and unknown as to how they correlate to the in vivo scenario, albeit efforts are underway to address this knowledge gap [101]. Such systems have recently been shown to be advantageous in determining the hazard posed by nanocellulose [47], as well as its interaction with cellular systems [102]. 
Table 2. An overview over the published studies focussing on the hazard assessment of nanocellulose. Details regarding the specific form of nanocellulose used, the biological system employed and the specific biochemical endpoint analysed are given.

\begin{tabular}{|c|c|c|c|}
\hline $\begin{array}{l}\text { Nanocellulose Form } \\
\text { Studied }\end{array}$ & Biological Model Used & Endpoint Assessed & Reference \\
\hline $\begin{array}{l}\text { Bacterial cellulose } \\
\text { nanofibres (BC-NF) }\end{array}$ & 3T3 fibroblasts, $\mathrm{CHO}$ cells & $\begin{array}{l}\text { mutagenicity, } \\
\text { proliferation, genotoxicity }\end{array}$ & [103] \\
\hline $\begin{array}{l}\text { Bacterial cellulose } \\
\text { nanofibres }\end{array}$ & HUVEC, C57/Bl6 mice & $\begin{array}{l}\text { viability, cytotoxicity, } \\
\text { apoptosis/necrosis, } \\
\text { cell cycle }\end{array}$ & [104] \\
\hline $\begin{array}{l}\text { Cellulose nanocrystals } \\
\text { (CNCs) }\end{array}$ & $\begin{array}{l}\text { Oncorhynchus mykiss } \\
\text { hepatocytes, Daphnia magna, } \\
\text { Ceriodaphia dubia, Pimephales } \\
\text { promelas, Vibrio fischeri, } \\
\text { Pseudokirchneriella subcapitata, } \\
\text { Hydra attenuata, Danio rerio }\end{array}$ & $\begin{array}{l}\text { genotoxicity, } \\
\text { reproduction, } \\
\text { survival, growth }\end{array}$ & [105] \\
\hline CNCs isolated from flay & HEK 293, Sf9 cells & uptake, cytotoxicity & [106] \\
\hline $\begin{array}{l}\text { CNCs isolated from cotton } \\
\text { and tunicates }\end{array}$ & $\begin{array}{l}\text { 3D model of the pulmonary } \\
\text { epithelial airway barrier }\end{array}$ & $\begin{array}{l}\text { cytotoxicity, } \\
\text { (pro)inflammatory } \\
\text { response }\end{array}$ & [93] \\
\hline $\begin{array}{l}\text { Cellulose nanofibers } \\
\text { isolated from } \\
\text { caraua/cotton }\end{array}$ & $\begin{array}{l}\text { Allium cepa, primary } \\
\text { lymphocytes, 3T3 fibroblasts }\end{array}$ & Genotoxicity & [107] \\
\hline Plant derived CNCs & $\begin{array}{l}\text { HBMEC, bEnd.3, RAW 264.7, } \\
\text { MCF-10A, MDA-MB-231, } \\
\text { MDA-MB-468, KB, } \\
\text { PC-3, C6 cells }\end{array}$ & uptake, cytotoxicity & [108] \\
\hline $\begin{array}{l}\text { Nanofibrillated cellulose } \\
\text { (NFC) }\end{array}$ & BEAS 2B cells & Genotoxicity & [109] \\
\hline $\begin{array}{l}\text { CNCs isolated from cotton, } \\
\text { flax, hemp }\end{array}$ & V79 fibroblast, Sf9 cells & Cytotoxicity & [110] \\
\hline $\begin{array}{l}\text { Cotton cellulose } \\
\text { nanofibres (CNF) }\end{array}$ & Bovine fibroblasts & $\begin{array}{l}\text { cytotoxicity, stress } \\
\text { response, apoptosis }\end{array}$ & [111] \\
\hline CNCs isolated from cotton & $\begin{array}{l}\text { BEAS 2B cells, } \\
\text { monocyte-derived } \\
\text { macrophages }\end{array}$ & $\begin{array}{l}\text { cytotoxicity, genotoxicity, } \\
\text { inflammatory response }\end{array}$ & [112] \\
\hline CNCs isolated from MCC & $\begin{array}{l}\text { NIH3T3 fibroblasts, } \\
\text { HCT116 cells }\end{array}$ & cell viability & [113] \\
\hline CNFs isolated from cotton & Chlorella vulgaris & cell viability, growth & {$[114]$} \\
\hline CNCs isolated from wood & C57BL/6 mice & pulmonary outcome & [115] \\
\hline
\end{tabular}

Whilst the specific mechanisms associated with and driving any of the observed biochemical and biomolecular reactions measured are a necessity, it is vital that a specific understanding of how nanocellulose interacts with different biological systems can be developed, and how this relates to the biochemical response measured. 
The use of state-of-the-art microscopy approaches will be necessary due to the innate difficulties in identifying nanocellulose within cellular structures. A recent study by Endes et al. showed the possibility to achieve imaging of CNCs within cells by fluorescently labelling the nanocellulose used, with the assumption that small amounts of dye, in this case fluorescein, does not have any significant effect on the associated tests, such as uptake or cytotoxicity [102]. Analytical techniques should be sought out, which can directly identify cellulose, without the need of modification to easily identify nanocellulose inside cells so that a toxicodynamic approach can be undertaken. Finally, once all this information is gained, then correlatory analysis against the specific physical and chemical characteristics of the nanocellulose sample so that efforts can be made to negate the production of such materials exhibiting these characteristics (i.e., safe-by-design nanomaterials).

It should be noted, that this article focuses on cellulouse nanocrystals. One of the greatest challenges in addressing the effect of nanocellulose, is that it comes in many different forms, not only with respect to source, aspect ratio and surface chemistry, and processing methods. These different forms usually focus on different systems, in different ways. Although the above table suggests the majority of research is focused on CNCs, there are others, which is beyond the scope of this paper. Nonetheless, the discussion contained within the present perspective would certainly fit towards any and all nanocellulose types.

\section{Summary and Outlook}

Nanocellulose is an interesting tool for material scientists as the platform to engineer desired functions into polymeric and biological systems. Many research groups have found a plethora of ways to use cellulose in both its bare, and functionalized form. These applications have shown that both in vitro and in vivo applications are viable, and do not create any measurable negative effects. Along with its production/isolation, the commercialization of products containing nanocellulose has begun and is constantly increasing. Several avenues of use seem to be emerging for nanocellulose, from high end smart and biocompatible materials, to large-scale use in commodity products. All these exciting properties of cellulose nanocrystals and cellulose based nanomaterials seem to be to the beginning of a new concept of enhanced commodity materials and specialized biomedicine in which materials science and biology are closely related; giving the opportunity to engineer the desired material using nanomaterials.

With all of these obvious advantages, there remains a lack of knowledge concerning the potential hazard nanocellulose may pose to human health, and furthermore at how, if, and at what dose humans would be exposed, given the wide range of potential life-cycle scenarios. Although there has been much research-based emphasis on deducing this unknown, and so far no adverse acute effects have been 
reported when using realistic concentrations, a variety of compounding factors disallow any meaningful wide-ranging understanding to be gained from the research currently available. Varying characteristics of the investigated sample deriving from differing production protocols, sources, dimensions, purity, concentration, application mode and exposure time can strongly influence the biological response observed in vitro or in vivo and may not reflect a realistic assessment of the potential hazard of cellulosic fibres in general and in particular for cellulose nanocrystals.

Therefore, in order to realize these aspects, and overcome these issues, it is suggested to consider the following points in order to fully, and holistically deduce the potential human health risk of nanocellulose;

- Assess and quantify what and if the released dose at each stage of the material's life-cycle is a potential mode for environmental as well as human exposure (e.g., inhalation and skin contact).

- At each stage of the life-cycle of nanocellulose undertaken, thorough characterisation of the released nanomaterial (if any) and decipher between single nanocellulose nanofibers, polymer composite released nanocellulose nanofibers and micron-sized particles. Several parameters need to be analyzed, the most relevant factors being: the dimensions (width, length, aspect ratio), colloidal stability on the studied medium, surface chemistry, specific surface area and degree of crystallinity (directly related to the stiffness of the material).

- In order to achieve the characterisation of the materials at every life-cycle stage, reliable and representative methods must be used (as suggested in Table 1). The need to develop alternative or adapted methods for every nanomaterial, especially nanocellulose remains and is the responsibility of the field to progress. New protocols need to be established for the facile characterization and determination of nanoparticle size and determination of surface chemistry on the nanoscale, which allow for a simple and realistic comparison between studies.

- Understanding of the acute and chronic effects of nanocellulose exposure, particularly during occupational exposure (i.e., isolation stage) in order to comprehend the ability for nanocellulose to either contribute to, or exacerbate pre-existing disease states.

- Determine the biomolecular and biochemical mechanisms that drive, if any, the (adverse) biological effects following nanocellulose exposure.

- The application of realistic doses in contrast to overload situations on target organ (in vitro) or related systems has to be the aim in any hazard assessment study.

- Relate the exposure dose effect and associated biochemical effects to the specific characteristics of the nanocellulose investigated in order to determine the specific 
physical and/or chemical characteristics that might be driving the possible hazardous response measured.

It is the hope that such suggestions towards the assessment of biological interactions and impact of nanocellulose to human health provides coherent and effective knowledge and understanding that can be put towards the development of regulatory guidelines for the production, use and disposal of nanocellulose. Further to this, elucidation of the biological impact of nanocellulose will only serve towards realizing the plethora of advantages posed by this naturally occurring material.

Acknowledgments: The authors gratefully acknowledge funding from the Swiss National Science Foundation (SNSF) and the SNSF National Research Programme 64 (406440-131264/1), the Adolphe Merkle Foundation and the U.S. Endowment for Forestry and Communities ( $\mathrm{P}^{3} \mathrm{Nano}$ ). Author EJF is visiting Professor in LabEx Tec 21, Grenoble, France (Investissements $\mathrm{d}^{\prime}$ Avenir-grant agreement $\mathrm{n}^{\circ}$ ANR-11-LABX-0030). All authors further thank the members of the Adolphe Merkle Institute for scientific discussion surrounding this topic matter.

Author Contributions: All authors conceived the idea for the perspective article and contributed to writing the manuscript. All authors critically reviewed and edited the manuscript.

Conflicts of Interest: The authors declare no conflict of interest.

\section{References}

1. Voet, D.; Voet, J.G. Biochemistry, 4th ed.; John Wiley \& Sons Inc.: Hoboken, NJ, USA, 2010.

2. Moon, R.J.; Martini, A.; Nairn, J.; Simonsen, J.; Youngblood, J. Cellulose nanomaterials review: Structure, properties and nanocomposites. Chem. Soc. Rev. 2011, 40, 3941-3994.

3. Eichhorn, S.J.; Dufresne, A.; Aranguren, M.; Marcovich, N.E.; Capadona, J.R.; Rowan, S.J.; Weder, C.; Thielemans, W.; Roman, M.; Renneckar, S.; et al. Review: Current international research into cellulose nanofibres and nanocomposites. J. Mater. Sci. 2010, 45, 1-33.

4. Araki, J.; Wada, M.; Kuga, S.; Okano, T. Flow properties of microcrystalline cellulose suspension prepared by acid treatment of native cellulose. Colloids Surf. A Physicochem. Eng. Asp. 1998, 142, 75-82.

5. Dong, X.; Revol, J.-F.; Gray, D. Effect of microcrystallite preparation conditions on the formation of colloid crystals of cellulose. Cellulose 1998, 5, 19-32.

6. Camarero-Espinosa, S.; Kuhnt, T.; Foster, E.J.; Weder, C. Isolation of thermally stable cellulose nanocrystals by phosphoric acid hydrolysis. Biomacromolecules 2013, 14, 1223-1230.

7. Jorfi, M.; Roberts, M.N.; Foster, E.J.; Weder, C. Physiologically responsive, mechanically adaptive bio-nanocomposites for biomedical applications. ACS Appl. Mater. Interfaces 2013, 5, 1517-1526.

8. Mueller, S.; Weder, C.; Foster, E.J. Isolation of cellulose nanocrystals from pseudostems of banana plants. RSC Adv. 2014, 4, 907-915.

9. De Souza Lima, M.M.; Wong, J.T.; Paillet, M.; Borsali, R.; Pecora, R. Translational and rotational dynamics of rodlike cellulose whiskers. Langmuir 2002, 19, 24-29. 
10. Jorfi, M.; Foster, E.J. Recent advances in nanocellulose for biomedical applications. J. Appl. Polym. Sci. 2015, 132, 41719.

11. Lin, N.; Dufresne, A. Nanocellulose in biomedicine: Current status and future prospect. Eur. Polym. J. 2014, 59, 302-325.

12. Camarero-Espinosa, S.; Rothen-Rutishauser, B.; Weder, C.; Foster, E.J. Directed cell growth in multi-zonal scaffolds for cartilage tissue engineering. Biomaterials 2016, 74, 42-52.

13. Biyani, M.V.; Foster, E.J.; Weder, C. Light-healable supramolecular nanocomposites based on modified cellulose nanocrystals. ACS Macro Lett. 2013, 2, 236-240.

14. Padalkar, S.; Capadona, J.R.; Rowan, S.J.; Weder, C.; Won, Y.-H.; Stanciu, L.A.; Moon, R.J. Natural biopolymers: Novel templates for the synthesis of nanostructures. Langmuir 2010, 26, 8497-8502.

15. Van den Berg, O.; Schroeter, M.; Capadona, J.R.; Weder, C. Nanocomposites based on cellulose whiskers and (semi)conducting conjugated polymers. J. Mater. Chem. 2007, 17, 2746-2753.

16. Gawryla, M.D.; van den Berg, O.; Weder, C.; Schiraldi, D.A. Clay aerogel/cellulose whisker nanocomposites: A nanoscale wattle and daub. J. Mater. Chem. 2009, 19, 2118-2124.

17. Li, Y.; Ren, H.; Ragauskas, A.J. Rigid polyurethane foam/cellulose whisker nancomposites: Preparation, characterization and properties. J. Nanosci. Nanotechnol. 2011, 11, 6904-6911.

18. Heath, L.; Thielemans, W. Cellulose nanowhisker aerogels. Green Chem. 2010, 12, 1448-1453.

19. Capadona, J.R.; Shanmuganathan, K.; Tyler, D.J.; Rowan, S.J.; Weder, C. Stimuli-responsive polymer nanocomposites inspired by the sea cucumber dermis. Science 2008, 319, 1370-1374.

20. Shanmuganathan, K.; Capadona, J.R.; Rowan, S.J.; Weder, C. Bio-inspired mechanically-adaptive nanocomposites derived from cotton cellulose whiskers. J. Mater. Chem. 2010, 20, 180-186.

21. Shanmuganathan, K.; Capadona, J.R.; Rowan, S.J.; Weder, C. Biomimetic mechanically adaptive nanocomposites. Prog. Polym. Sci. 2010, 35, 212-222.

22. Rusli, R.; Shanmuganathan, K.; Rowan, S.J.; Weder, C.; Eichhorn, S.J. Stress-transfer in anisotropic and environmentally adaptive cellulose whisker nanocomposites. Biomacromolecules 2010, 11, 762-768.

23. Mendez, J.; Annamalai, P.K.; Eichhorn, S.J.; Rusli, R.; Rowan, S.J.; Foster, E.J.; Weder, C. Bioinspired mechanically adaptive polymer nanocomposites with water-activated shape-memory effect. Macromolecules 2011, 44, 6827-6835.

24. Dagnon, K.L.; Shanmuganathan, K.; Weder, C.; Rowan, S.J. Water-triggered modulus changes of cellulose nanofiber nanocomposites with hydrophobic polymer matrices. Macromolecules 2012, 45, 4707-4715.

25. Way, A.E.; Hsu, L.; Shanmuganathan, K.; Weder, C.; Rowan, S.J. Ph-responsive cellulose nanocrystal gels and nanocomposites. ACS Macro Lett. 2012, 1, 1001-1006. 
26. Shatkin, J.A.; Wegner, T.H.; Bilek, E.T.; Cowie, J. Market projections of cellulose nanomaterial-enabled products-part 1: Applications. Tappi J. 2014, 13, 9-16.

27. Davis, C.S.; Grolman, D.L.; Karim, A.; Gilman, J.W. What do we still need to understand to commercialize cellulose nanomaterials? Green Mater. 2015, 3, 53-58.

28. Stone, V.; Pozzi-Mucelli, S.; Tran, L.; Aschberger, K.; Sabella, S.; Vogel, U.; Poland, C.; Balharry, D.; Fernandes, T.; Gottardo, S.; et al. Its-nano-Prioritising nanosafety research to develop a stakeholder driven intelligent testing strategy. Part. Fibre Toxicol. 2014, $11,1-12$.

29. Krug, H.F. Nanosafety research-Are we on the right track? Angew. Chem. Int. Ed. 2014, 53, 12304-12319.

30. Arts, J.H.E.; Hadi, M.; Irfan, M.-A.; Keene, A.M.; Kreiling, R.; Lyon, D.; Maier, M.; Michel, K.; Petry, T.; Sauer, U.G.; et al. A decision-making framework for the grouping and testing of nanomaterials (DF4nanoGrouping). Regul. Toxicol. Pharmacol. 2015, 71, S1-S27.

31. Shatkin, J.A.; Kim, B. Cellulose nanomaterials: Life cycle risk assessment, and environmental health and safety roadmap. Environ. Sci. Nano 2015, 2, 477-499.

32. Nowack, B.; Brouwer, C.; Geertsma, R.E.; Heugens, E.H.W.; Ross, B.L.; Toufektsian, M.-C.; Wijnhoven, S.W.P.; Aitken, R.J. Analysis of the occupational, consumer and environmental exposure to engineered nanomaterials used in 10 technology sectors. Nanotoxicology 2012, 7, 1152-1156.

33. Rusli, R.; Eichhorn, S.J. Determination of the stiffness of cellulose nanowhiskers and the fiber-matrix interface in a nanocomposite using raman spectroscopy. Appl. Phys. Lett. 2008, 93, 033111.

34. Sturcova, A.; Davies, G.R.; Eichhorn, S.J. Elastic modulus and stress-transfer properties of tunicate cellulose whiskers. Biomacromolecules 2005, 6, 1055-1061.

35. Favier, V.; Chanzy, H.; Cavaille, J.Y. Polymer nanocomposites reinforced by cellulose whiskers. Macromolecules 1995, 28, 6365-6367.

36. Losert, S.; von Goetz, N.; Bekker, C.; Fransman, W.; Wijnhoven, S.W.P.; Delmaar, C.; Hungerbuhler, K.; Ulrich, A. Human exposure to conventional and nanoparticlecontaining sprays-A critical review. Environ. Sci. Technol. 2014, 48, 5366-5378.

37. Maynard, A.D.; Baron, P.A.; Foley, M.; Shvedova, A.A.; Kisin, E.R.; Castranova, V. Exposure to carbon nanotube material: Aerosol release during the handling of unrefined single-walled carbon nanotube material. J. Toxicol. Environ. Health A 2004, 67, 87-107.

38. Lee, J.S.; Choi, Y.C.; Shin, J.H.; Lee, J.H.; Lee, Y.; Park, S.Y.; Baek, J.E.; Park, J.D.; Ahn, K.; $\mathrm{Yu}, \mathrm{I}$.J. Health surveillance study of workers who manufacture multi-walled carbon nanotubes. Nanotoxicology 2015, 9, 802-811.

39. Zhang, Q.; Huang, J.-Q.; Zhao, M.-Q.; Qian, W.-Z.; Wei, F. Carbon nanotube mass production: Principles and processes. ChemSusChem 2011, 4, 864-889.

40. Kobayashi, H.; Kanoh, S.; Motoyoshi, K.; Aida, S. Diffuse lung disease caused by cotton fibre inhalation but distinct from byssinosis. Thorax 2004, 59, 1095-1097. 
41. Steffi, F.; Jurgen, S. Environmental, health and safety aspects of nanotechnology-Implications for the R\&D in (small) companies. Sci. Technol. Adv. Mater. 2007, 8, 12-18.

42. Kuhlbusch, T.A.J.; Asbach, C.; Fissan, H.; Göhler, D.; Stintz, M. Nanoparticle exposure at nanotechnology workplaces: A review. Part. Fibre Toxicol. 2011, 8, 22.

43. Pietroiusti, A.; Magrini, A. Engineered nanoparticles at the workplace: Current knowledge about workers' risk. Occup. Med. 2014, 64, 319-330.

44. Methner, M.; Hodson, L.; Geraci, C. Nanoparticle emission assessment technique (NEAT) for the identification and measurement of potential inhalation exposure to engineered nanomaterials-Part A. J. Occup. Environ. Hyg. 2009, 7, 127-132.

45. NIOSH. Current Intelligence Bulletin 65: Occupational Exposure to Carbon Nanotubes and Nanofibers. Available online: http://www.cdc.gov/niosh/docs/2013-145/ (accessed on 12 September 2015).

46. Morrow, P.E. Possible mechanisms to explain dust overloading of the lungs. Fundam. Appl. Toxicol. 1988, 10, 369-384.

47. Endes, C.; Schmid, O.; Kinnear, C.; Mueller, S.; Camarero-Espinosa, S.; Vanhecke, D.; Foster, E.J.; Petri-Fink, A.; Rothen-Rutishauser, B.; Weder, C.; et al. An in vitro testing strategy towards mimicking the inhalation of high aspect ratio nanoparticles. Part. Fibre Toxicol. 2014, 11, 1-13.

48. Morris, J.; Willis, J.; De Martinis, D.; Hansen, B.; Laursen, H.; Sintes, J.R.; Kearns, P.; Gonzalez, M. Science policy considerations for responsible nanotechnology decisions. Nat. Nanotechnol. 2011, 6, 73-77.

49. NIOSH. Niosh Potential Occupational Carcinogens. Available online: http://www.cdc. gov/niosh/npg/nengapdxa.html (accessed on 12 September 2015).

50. Borm, P.J.; Tran, L.; Donaldson, K. The carcinogenic action of crystalline silica: A review of the evidence supporting secondary inflammation-driven genotoxicity as a principal mechanism. Crit. Rev. Toxicol. 2011, 41, 756-770.

51. Thompson, D.; Chen, S.-C.; Wang, J.; Pui, D.Y.H. Aerosol emission monitoring and assessment of potential exposure to multi-walled carbon nanotubes in the manufacture of polymer nanocomposites. Ann. Occup. Hyg. 2015, 59, 1135-1151.

52. Schlagenhauf, L.; Chu, B.T.T.; Buha, J.; Nüesch, F.; Wang, J. Release of carbon nanotubes from an epoxy-based nanocomposite during an abrasion process. Environ. Sci. Technol. 2012, 46, 7366-7372.

53. Schlagenhauf, L.; Nüesch, F.; Wang, J. Release of carbon nanotubes from polymer nanocomposites. Fibers 2014, 2, 108.

54. Bergin, I.L.; Witzmann, F.A. Nanoparticle toxicity by the gastrointestinal route: Evidence and knowledge gaps. Int. J. Biomed. Nanosci. Nanotechnol. 2013, 3, 163-210.

55. Muthu, M.S.; Leong, D.T.; Mei, L.; Feng, S.-S. Nanotheranostics-Application and further development of nanomedicine strategies for advanced theranostics. Theranostics 2014, 4 , 660-677. 
56. Walser, T.; Limbach, L.K.; Brogioli, R.; Erismann, E.; Flamigni, L.; Hattendorf, B.; Juchli, M.; Krumeich, F.; Ludwig, C.; Prikopsky, K.; et al. Persistence of engineered nanoparticles in a municipal solid-waste incineration plant. Nat. Nanotechnol. 2012, 7, 520-524.

57. Oberdorster, G.; Oberdorster, E.; Oberdorster, J. Nanotoxicology: An emerging discipline evolving from studies of ultrafine particles. Environ Health Perspect. 2005, 113, 823-839.

58. Bouwmeester, H.; Lynch, I.; Marvin, H.J.; Dawson, K.A.; Berges, M.; Braguer, D.; Byrne, H.J.; Casey, A.; Chambers, G.; Clift, M.J.; et al. Minimal analytical characterization of engineered nanomaterials needed for hazard assessment in biological matrices. Nanotoxicology 2011, 5, 1-11.

59. Duffin, R.; Tran, L.; Brown, D.; Stone, V.; Donaldson, K. Proinflammogenic effects of low-toxicity and metal nanoparticles in vivo and in vitro: Highlighting the role of particle surface area and surface reactivity. Inhal. Toxicol. 2007, 19, 849-856.

60. Lin, P.-C.; Lin, S.; Wang, P.C.; Sridhar, R. Techniques for physicochemical characterization of nanomaterials. Biotechnol. Adv. 2014, 32, 711-726.

61. Moore, T.L.; Rodriguez-Lorenzo, L.; Hirsch, V.; Balog, S.; Urban, D.; Jud, C.; Rothen-Rutishauser, B.; Lattuada, M.; Petri-Fink, A. Nanoparticle colloidal stability in cell culture media and impact on cellular interactions. Chem. Soc. Rev. 2015, 44, 6287-6305.

62. Balog, S.; Rodriguez-Lorenzo, L.; Monnier, C.A.; Obiols-Rabasa, M.; Rothen-Rutishauser, B.; Schurtenberger, P.; Petri-Fink, A. Characterizing nanoparticles in complex biological media and physiological fluids with depolarized dynamic light scattering. Nanoscale 2015, 7, 5991-5997.

63. Donaldson, K.; Murphy, F.A.; Duffin, R.; Poland, C.A. Asbestos, carbon nanotubes and the pleural mesothelium: A review of the hypothesis regarding the role of long fibre retention in the parietal pleura, inflammation and mesothelioma. Part. Fibre Toxicol. 2010, 7 .

64. Ellouk, S.A.; Jaurand, M.C. Review of animal/in vitro data on biological effects of man-made fibers. Environ Health Perspect. 1994, 102, 47-61.

65. Davis, J.; Addison, J.; Bolton, R.; Donaldson, K.; Jones, A.; Smith, T. The pathogenicity of long versus short fibre samples of amosite asbestos administered to rats by inhalation and intraperitoneal injection. Br. J. Exp. Pathol. 1986, 67, 415-430.

66. Stanton, M.F.; Layard, M.; Tegeris, A.; Miller, E.; May, M.; Kent, E. Carcinogenicity of fibrous glass: Pleural response in the rat in relation to fiber dimension. J. Natl. Cancer Inst. 1977, 58, 587-603.

67. Abbate, S.; Martino, L.B.; Tringali, M.A.; Catania, S.; Albiero, F.; Cavallari, V.; Costa, C.; Giacobbe, G.; Brecciaroli, R.; Giorgianni, C.; et al. Changes induced by exposure of the human lung to glass fibre reinforced plastic. Environ Health Perspect. 2006, 114, 1725-1729.

68. Poland, C.A.; Duffin, R.; Kinloch, I.; Maynard, A.; Wallace, W.A.H.; Seaton, A.; Stone, V.; Brown, S.; MacNee, W.; Donaldson, K. Carbon nanotubes introduced into the abdominal cavity of mice show asbestos-like pathogenicity in a pilot study. Nat. Nanotechnol. 2008, $3,423-428$. 
69. Schinwald, A.; Chernova, T.; Donaldson, K. Use of silver nanowires to determine thresholds for fibre length-dependent pulmonary inflammation and inhibition of macrophage migration in vitro. Part. Fibre Toxicol. 2012, 9, 1-15.

70. Sacui, I.A.; Nieuwendaal, R.C.; Burnett, D.J.; Stranick, S.J.; Jorfi, M.; Weder, C.; Foster, E.J.; Olsson, R.T.; Gilman, J.W. Comparison of the properties of cellulose nanocrystals and cellulose nanofibrils isolated from bacteria, tunicate, and wood processed using acid, enzymatic, mechanical, and oxidative methods. ACS Appl. Mater. Interfaces 2014, 6, 6127-6138.

71. Shanmuganathan, K.; Capadona, J.R.; Rowan, S.J.; Weder, C. Stimuli-responsive mechanically adaptive polymer nanocomposites. ACS Appl. Mater. Interfaces 2010, 2, 165-174.

72. Utembe, W.; Potgieter, K.; Stefaniak, A.B.; Gulumian, M. Dissolution and biodurability: Important parameters needed for risk assessment of nanomaterials. Part. Fibre Toxicol. 2015, 12, 1-12.

73. Michen, B.; Geers, C.; Vanhecke, D.; Endes, C.; Rothen-Rutishauser, B.; Balog, S.; Petri-Fink, A. Avoiding drying-artifacts in transmission electron microscopy: Characterizing the size and colloidal state of nanoparticles. Sci. Rep. 2015, 5, 9793.

74. Dammak, A.; Moreau, C.; Beury, N.; Schwikal, K.; Winter Heiko, T.; Bonnin, E.; Saake, B.; Cathala, B. Elaboration of multilayered thin films based on cellulose nanocrystals and cationic xylans: Application to xylanase activity detection. Holzforschung 2013, 67, 579-586.

75. Beck-Candanedo, S.; Roman, M.; Gray, D.G. Effect of reaction conditions on the properties and behavior of wood cellulose nanocrystal suspensions. Biomacromolecules 2005, 6, 1048-1054.

76. Lahiji, R.R.; Xu, X.; Reifenberger, R.; Raman, A.; Rudie, A.; Moon, R.J. Atomic force microscopy characterization of cellulose nanocrystals. Langmuir 2010, 26, 4480-4488.

77. Podsiadlo, P.; Choi, S.-Y.; Shim, B.; Lee, J.; Cuddihy, M.; Kotov, N.A. Molecularly engineered nanocomposites: Layer-by-layer assembly of cellulose nanocrystals. Biomacromolecules 2005, 6, 2914-2918.

78. Mandal, A.; Chakrabarty, D. Isolation of nanocellulose from waste sugarcane bagasse (scb) and its characterization. Carbohydr. Polym. 2011, 86, 1291-1299.

79. Boluk, Y.; Danumah, C. Analysis of cellulose nanocrystal rod lengths by dynamic light scattering and electron microscopy. J. Nanopart. Res. 2013, 16, 1-7.

80. Bondeson, D.; Mathew, A.; Oksman, K. Optimization of the isolation of nanocrystals from microcrystalline cellulose by acid hydrolysis. Cellulose 2006, 13, 171-180.

81. Dumanli, A.G.; van der Kooij, H.M.; Kamita, G.; Reisner, E.; Baumberg, J.J.; Steiner, U.; Vignolini, S. Digital color in cellulose nanocrystal films. ACS Appl. Mater. Interfaces 2014, 6, 12302-12306.

82. Beck, S.; Bouchard, J.; Berry, R. Dispersibility in water of dried nanocrystalline cellulose. Biomacromolecules 2012, 13, 1486-1494. 
83. Morandi, G.; Heath, L.; Thielemans, W. Cellulose nanocrystals grafted with polystyrene chains through surface-initiated atom transfer radical polymerization (SI-ATRP). Langmuir 2009, 25, 8280-8286.

84. Junior de Menezes, A.; Siqueira, G.; Curvelo, A.A.S.; Dufresne, A. Extrusion and characterization of functionalized cellulose whiskers reinforced polyethylene nanocomposites. Polymer 2009, 50, 4552-4563.

85. Biyani, M.; Weder, C.; Foster, E.J. Photoswitchable nanocomposites made from coumarin-functionalized cellulose nanocrystals. Polym. Chem. 2014, 5, 5501-5508.

86. Habibi, Y.; Goffin, A.L.; Schiltz, N.; Duquesne, E.; Dubois, P.; Dufresne, A. Bionanocomposites based on poly(epsilon-caprolactone)-grafted cellulose nanocrystals by ring-opening polymerization. J. Mater. Chem. 2008, 18, 5002-5010.

87. Lu, P.; Hsieh, Y.-L. Preparation and properties of cellulose nanocrystals: Rods, spheres, and network. Carbohydr. Polym. 2010, 82, 329-336.

88. Shin, Y.; Exarhos, G.J. Template synthesis of porous titania using cellulose nanocrystals. Mater. Lett. 2007, 61, 2594-2597.

89. Beck-Candanedo, S.; Viet, D.; Gray, D. Induced phase separation in cellulose nanocrystal suspensions containing ionic dye species. Cellulose 2006, 13, 629-635.

90. Cullen, R.T.; Miller, B.G.; Jones, A.D.; Davis, J.M.G. Toxicity of cellulose fibres. Ann. Occup. Hyg. 2002, 46, 81-84.

91. Milton, D.K.; Godleski, J.J.; Feldman, H.A.; Greaves, I.A. Toxicity of intratracheally instilled cotton dust, cellulose, and endotoxin. Am. Rev. Respir. Dis. 1990, 142, 184-192.

92. Cullen, R.T.; Searl, A.; Miller, B.G.; Davis, J.M.G.; Jones, A.D. Pulmonary and intraperitoneal inflammation induced by cellulose fibres. J. Appl. Toxicol. 2000, 20, $49-60$.

93. Clift, M.J.D.; Foster, E.J.; Vanhecke, D.; Studer, D.; Wick, P.; Gehr, P.; Rothen-Rutishauser, B.; Weder, C. Investigating the interaction of cellulose nanofibers derived from cotton with a sophisticated $3 \mathrm{~d}$ human lung cell coculture. Biomacromolecules 2011, 12, 3666-3673.

94. Roman, M. Toxicity of cellulose nanocrystals: A review. Ind. Biotechnol. 2015, 11, 25-33.

95. Manke, A.; Wang, L.; Rojanasakul, Y. Mechanisms of nanoparticle-induced oxidative stress and toxicity. BioMed Res. Int. 2013, 2013, 942916.

96. Magdolenova, Z.; Collins, A.; Kumar, A.; Dhawan, A.; Stone, V.; Dusinska, M. Mechanisms of genotoxicity. A review of in vitro and in vivo studies with engineered nanoparticles. Nanotoxicology 2014, 8, 233-278.

97. Burden, N.; Aschberger, K.; Chaudhry, Q.; Clift, M.J.D.; Doak, S.; Fowler, P.; Johnston, H.; Landsiedel, R.; Rowland, J.; Stone, V. Aligning nanotoxicology with the 3Rs: What is needed to realise the short, medium and long-term opportunities? Nano Today 2016, accepted.

98. Hartung, T.; Sabbioni, E. Alternative in vitro assays in nanomaterial toxicology. Wiley Interdiscip. Rev. Nanomed. Nanobiotechnol. 2011, 3, 545-573.

99. Fabbrizi, M.R.; Duff, T.; Oliver, J.; Wilde, C. Advanced in vitro systems for efficacy and toxicity testing in nanomedicine. Eur. J. Nanomed. 2014, 6, 171-183. 
100. Clift, M.J.; Endes, C.; Vanhecke, D.; Wick, P.; Gehr, P.; Schins, R.P.; Petri-Fink, A.; Rothen-Rutishauser, $\mathrm{B}$. A comparative study of different in vitro lung cell culture systems to assess the most beneficial tool for screening the potential adverse effects of carbon nanotubes. Toxicol. Sci. 2014, 137, 55-64.

101. Mihalchik, A.L.; Ding, W.; Porter, D.W.; McLoughlin, C.; Schwegler-Berry, D.; Sisler, J.D.; Stefaniak, A.B.; Snyder-Talkington, B.N.; Cruz-Silva, R.; Terrones, M.; et al. Effects of nitrogen-doped multi-walled carbon nanotubes compared to pristine multi-walled carbon nanotubes on human small airway epithelial cells. Toxicology 2015, 333, 25-36.

102. Endes, C.; Mueller, S.; Kinnear, C.; Vanhecke, D.; Foster, E.J.; Petri-Fink, A.; Weder, C.; Clift, M.J.D.; Rothen-Rutishauser, B. Fate of cellulose nanocrystal aerosols deposited on the lung cell surface in vitro. Biomacromolecules 2015, 16, 1267-1275.

103. Moreira, S.; Silva, N.B.; Almeida-Lima, J.; Oliveira Rocha, H.A.; Batistuzzo Medeiros, S.R.; Alves, C., Jr.; Gama, F.M. Bc nanofibres: In vitro study of genotoxicity and cell proliferation. Toxicol. Lett. 2009, 189, 235-241.

104. Jeong, S.I.; Lee, S.E.; Yang, H.; Jin, Y.H.; Park, C.S.; Park, Y.S. Toxicologic evaluation of bacterial synthesized cellulose in endothelial cells and animals. Mol. Cell. Toxicol. 2010, 6, 373-380.

105. Kovacs, T.; Naish, V.; O'Connor, B.; Blaise, C.; Gagne, F.; Hall, L.; Trudeau, V.; Martel, P. An ecotoxicological characterization of nanocrystalline cellulose (ncc). Nanotoxicology 2010, 4, 255-270.

106. Mahmoud, K.A.; Mena, J.A.; Male, K.B.; Hrapovic, S.; Kamen, A.; Luong, J.H.T. Effect of surface charge on the cellular uptake and cytotoxicity of fluorescent labeled cellulose nanocrystals. ACS Appl. Mater. Interfaces 2010, 2, 2924-2932.

107. de Lima, R.; Mattoso, L.H.; Feitosa, L.O.; Maruyama, C.R.; Barga, M.A.; Yamawaki, P.C.; Vieira, I.J.; Teixeira, E.M.; Corrêa, A.C.; Fraceto, L.F. Evaluation of the genotoxicity of cellulose nanofibers. Int. J. Nanomed. 2012, 7, 3555-3565.

108. Dong, S.; Hirani, A.A.; Colacino, K.R.; Lee, Y.W.; Roman, M. Cytotoxicity and cellular uptake of cellulose nanocrystals. Nano LIFE 2012, 2, 1241006.

109. Hannukainen, K.S.; Suhonen, S.; Savolainen, K.; Norppa, H. Genotoxicity of nanofibrillated cellulose in vitro as measured by enzyme comet assay. Toxicol. Lett. 2012, 211, S71.

110. Male, K.B.; Leung, A.C.W.; Montes, J.; Kamen, A.; Luong, J.H.T. Probing inhibitory effects of nanocrystalline cellulose: Inhibition versus surface charge. Nanoscale 2012, 4 , 1373-1379.

111. Pereira, M.M.; Raposo, N.R.B.; Brayner, R.; Teixeira, E.M.; Oliveira, V.; Quintão, C.C.R.; Camargo, L.S.A.; Mattoso, L.H.C.; Brandão, H.M. Cytotoxicity and expression of genes involved in the cellular stress response and apoptosis in mammalian fibroblast exposed to cotton cellulose nanofibers. Nanotechnology 2013, 24, 075103.

112. Catalan, J.; Ilves, M.; Jarventaus, H.; Hannukainen, K.S.; Kontturi, E.; Vanhala, E.; Alenius, H.; Savolainen, K.M.; Norppa, H. Genotoxic and immunotoxic effects of cellulose nanocrystals in vitro. Environ. Mol. Mutagen. 2015, 56, 171-182. 
113. Hanif, Z.; Ahmed, F.R.; Shin, S.W.; Kim, Y.-K.; Um, S.H. Size- and dose-dependent toxicity of cellulose nanocrystals (CNC) on human fibroblasts and colon adenocarcinoma. Colloids Surf. B 2014, 119, 162-165.

114. Pereira, M.; Mouton, L.; Yepremian, C.; Coute, A.; Lo, J.; Marconcini, J.; Ladeira, L.; Raposo, N.; Brandao, H.; Brayner, R. Ecotoxicological effects of carbon nanotubes and cellulose nanofibers in chlorella vulgaris. J. Nanobiotechnol. 2014, 12, 15.

115. Yanamala, N.; Farcas, M.T.; Hatfield, M.K.; Kisin, E.R.; Kagan, V.E.; Geraci, C.L.; Shvedova, A.A. In vivo evaluation of the pulmonary toxicity of cellulose nanocrystals: A renewable and sustainable nanomaterial of the future. ACS Sustain. Chem. Eng. 2014, 2, 1691-1698. 

MDPI AG

St. Alban-Anlage 66

4052 Basel, Switzerland

Tel. +41616837734

Fax +41 613028918

http://www.mdpi.com

Fibers Editorial Office

E-mail: fibers@mdpi.com

http://www.mdpi.com/journal/fibers

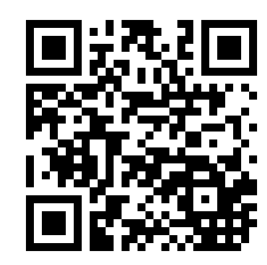



MDPI AG

St. Alban-Anlage 66

4052 Basel

Switzerland

Tel: +41 616837734

Fax: +41 613028918 